\title{
Search for the Higgs Boson Produced in Association with a Vector Boson Using Like-Sign Dilepton Events in 1.96-TeV Proton-Antiproton Collisions
}

A Dissertation Submitted to the Graduate School of Osaka City University in Partial Fulfillment of the Requirements for the Degree of Doctor of Philosophy in Science

\section{Daisuke Yamato}

\author{
January, 2013
}





\begin{abstract}
This thesis presents a search for the standard model and fermiophobic Higgs bosons produced in association with a vector boson using like-sign dilepton events collected by Collider Detector at Fermilab (CDF II). This analysis uses the data corresponding to an integrated luminosity of $9.4 \mathrm{fb}^{-1}$. The search is characterized by minimally biased cuts which maximize the acceptance of the Higgs bosons. The numbers of expected signal events passing requirements for like-sign dilepton are 5.6 for the fermiophobic Higgs on the mass of $110 \mathrm{GeV} / c^{2}$ assuming the standard model production cross section, and 1.7 for the standard model on the mass of $160 \mathrm{GeV} / c^{2}$. The expectation of background events are 696.1, while the number of observed events are 624 .

To discriminate signal events from backgrounds, the search employs a multivariate analysis based on boosted decision trees.

There are no significant disagreements in the BDT outputs, then, upper limits at a $95 \%$ confidence level are set on $\sigma(p \bar{p} \rightarrow V h) \times \mathcal{B}(h \rightarrow W W)$ for both Higgs bosons scenarios. The expected and observed limits are 5.9 and 9.2 , respectively, relative to the standard model expectation for the standard model Higgs boson mass of 160 $\mathrm{GeV} / c^{2}$. For the fermiophobic Higgs boson corresponding to the mass of $110 \mathrm{GeV} / c^{2}$, the expected and observed limits are 2.6 and 4.4 , respectively.
\end{abstract}





\section{Acknowledgment}

First of all, I would like to thank all of the people who supported me during the course of my Ph.D program.

I would like to thank my adviser, Prof. Yoshihiro Seiya. This work was heavily relied on his insight, advice, and many discussions with him. Under his guidance, I learned how to program and how to give a great presentation. I particularly appreciate the chance to I am greatful to Prof. Kazuhiro Yamamoto for advising the physics ideas which are necessary for my Ph.D works. Also, I have to appreciate Prof. Toru Okusawa. He is the first person who introduced me the experimental particle physics. I feel really grateful to Dr. Hirokazu Kobayashi, Takayuki Wakisaka, and Atsunari Hamaguchi. I have finished my work by incorporating and comparing their methods with my analysis.

I would like to thank the conveners of CDF Higgs group, Prof. Eric James, Craig Group, and Homer Wolfe. They gave me a lot of comments, questions, and suggestions about my analysis. Due to their contibutions, my analysis had been polished in each Higgs meeting.

I thank the all CDF members who taught and helped me during ACE shift, especially, Dee Hahn, Steve Hahn, JJ schmidt, Phil Schlabach, Ankush Mitra, William Budgett, Donatella Torretta, Pasha Murat, Benedetto Di Ruzza, and Saty Behari. Besides, I was often invited to a nice party, and it was always a great time with them.

I would like to express thanks Carol Picciolo, Sthephanie Schuler, Tanja Waltrip, Kyoko Kunori, and Kazuko Kumashiro, and Yukiko Kawaguchi. They supported me by a lot of their secretary works, therefore, I was able to concentrate on my working.

I thank the members of other institutions, Kiyomi Seiya, Syuichi Kunori, Kunitaka Kondo, Shinhong Kim, Fumihiko Ukegawa, Yuji Takeuchi, Makoto Shimojima, Yukihiro Kato, Kohei Yorita, Tomonobu Tomura, Hideki Miyake, Masakazu Kurata, Yoshikazu Nagai, Kenichi Takemasa, Yuji Sudo, and Yuki Sakurai. They gave me a lot of suggestions and it was a great time to discuss them.

I thank the Fermilab staff and the technical staffs of the participating institutions for their vital contributions. This work was supported by the U.S. Department of Energy and National Science Foundation; the Italian Istituto Nazionale di Fisica Nucleare; the Ministry of Education, Culture, Sports, Science and Technology of Japan; the Natural Sciences and Engineering Research Council of Canada; the National Science Council of the Republic of China; the Swiss National Science Foundation; the A.P. Sloan Foundation; the Bundesministerium für Bildung und Forschung, Germany; the 
Korean World Class University Program, the National Research Foundation of Korea; the Science and Technology Facilities Council and the Royal Society, UK; the Russian Foundation for Basic Research; the Ministerio de Ciencia e Innovación, and Programa Consolider-Ingenio 2010, Spain; the Slovak R\&D Agency; the Academy of Finland; and the Australian Research Council (ARC).

Finally, I would like to thank my family, who always supported my interest in physics. 


\section{Contents}

$\begin{array}{ll}\text { Abstract } & \text { i }\end{array}$

Acknowledgment iii

1 Introduction $\quad 1$

1.1 The Standard Model . . . . . . . . . . . . . . . . . . . . . . 1

1.1.1 Elementary Particles in the Standard Model . . . . . . . . . . . 2

1.1.2 Quantum Electrodynamics: $U(1) \ldots \ldots . \ldots 3$

1.1.3 Quantum Chromodynamics: $S U(3)_{C} \ldots \ldots \ldots 5$

1.1.4 Electroweak Theory: $S U(2)_{L} \otimes U(1)_{Y} \ldots \ldots \ldots 6$

1.1.5 Spontaneous Symmetry Breaking . . . . . . . . . . . . 8

1.1.6 Higgs Mechanism . . . . . . . . . . . . . . . . . . . . . 9 9

1.1.7 Two Higgs Doublet Model . . . . . . . . . . . . . . . . . . . . . 12

1.2 Production of The Higgs Boson at Tevatron . . . . . . . . . . . . . . . 14

1.2.1 The gluon-gluon Fusion Production . . . . . . . . . . . . . 15

1.2.2 The Associated Production with Vector Boson . . . . . . . . . . 15

1.2.3 The Vector Boson Fusion Production . . . . . . . . . . . . . . . 15

1.2.4 The Associated Production with Heavy Quarks . . . . . . . . . 15

1.3 Decay of The Higgs Boson at Tevatron . . . . . . . . . . . . . . . . 16

1.3.1 $h \rightarrow f \bar{f}$ Mode . . . . . . . . . . . . . . . . 17

$1.3 .2 \quad h \rightarrow W W^{(*)}$ Mode . . . . . . . . . . . . . . . . . . . . . . . 17

1.3.3 $h \rightarrow \gamma \gamma$ Mode . . . . . . . . . . . . . . . . . . 17

1.4 Higgs Boson Mass Constraints . . . . . . . . . . . . . . . . . . . 17

1.4.1 Theoretical Higgs Boson Mass Constraints . . . . . . . . . . . . 18

1.4.2 Experimental Higgs Boson Mass Constraints . . . . . . . . . . . 21

1.5 Higgs Boson Searches at Tevatron . . . . . . . . . . . . . . . . 24

1.6 Observation of a New Particle at the LHC . . . . . . . . . . . . . . . 25

2 Experimental Apparatus $\quad 29$

2.1 The Accelerator Complex . . . . . . . . . . . . . . . . . . . 29

2.1.1 Proton Production and Boosting . . . . . . . . . . . . . 29

2.1 .2 Main Injector . . . . . . . . . . . . . . . . . . . . . . . . . . . . . . . . . . 30

2.1.3 Antiproton Source . . . . . . . . . . . . . . . . 30 
2.1 .4 Recycler . . . . . . . . . . . . . . . . . . . . . . 31

2.1.5 Tevatron . . . . . . . . . . . . . . . . . . . . 31

2.1 .6 Luminosity . . . . . . . . . . . . . . . . . . . . . . 32

2.2 The Collider Detector at Fermilab . . . . . . . . . . . . . . . 32

2.3 Coordinate System in the CDF . . . . . . . . . . . . . . . . 33

2.4 Tracking Systems . . . . . . . . . . . . . . . . . . . . . . . . . 34

$2.4 .1 \quad$ Layer $00 \ldots \ldots \ldots \ldots \ldots$

2.4.2 Silicon Vertex Detector . . . . . . . . . . . . . . . . . 35

2.4.3 Intermediate Silicon Layers _ . . . . . . . . . . . . . 36

2.4.4 Central Outer Tracker . . . . . . . . . . . . . . . . . . 37

2.5 Calorimeter Systems . . . . . . . . . . . . . . . . . . . . . . . . . 39

2.5.1 Central Calorimeter . . . . . . . . . . . . . . . . . 40

2.5.2 Plug Calorimeter . . . . . . . . . . . . . . . . . . . . . 41

2.6 Muon Detectors . . . . . . . . . . . . . . . . . . . . . 42

2.7 Luminosity Monitor . . . . . . . . . . . . . . . . . . . . . . 43

2.8 Trigger Systems . . . . . . . . . . . . . . . . . . . . . . . 45

$2.8 .1 \quad$ Level-1 . . . . . . . . . . . . . . . . . . . . . 46

2.8 .2 Level-2 . . . . . . . . . . . . . . . . . . . . . . 47

$2.8 .3 \quad$ Level-3 . . . . . . . . . . . . . . . . . . . . . . . . 48

3 Dataset and Event Selection 49

3.1 Dataset and Triggers . . . . . . . . . . . . . . . . . . . . . . 49

3.2 Event Selection . . . . . . . . . . . . . . . . . . . 50

3.2 .1 Pre-Event Selection . . . . . . . . . . . . . . . . . 50

3.2 .2 Lepton Identification . . . . . . . . . . . . . . . . . 50

3.2 .3 Jet Reconstruction . . . . . . . . . . . . . . . . . . . . 55

3.2.4 Missing Transverse Energy . . . . . . . . . . . . . . . . 58

3.3 Like-Sign Dilepton Event Selection _. . . . . . . . . . . . . . 59

4 Background Estimation $\quad 67$

4.1 Residual Photon-Conversions . . . . . . . . . . . . . . . . . 67

4.1.1 Photon-Conversion Detection Efficiency . . . . . . . . . . . 67

4.1 .2 Data Samples . . . . . . . . . . . . . . . . . . . 68

4.1 .3 Monte Carlo Tuning . . . . . . . . . . . . . . . . . . 70

4.1.4 Relative Efficiency in Low $p_{T}$ Region $\ldots \ldots \ldots \ldots$. . . . . 70

4.1.5 Absolute Efficiency on the Plateau . . . . . . . . . . . 76

4.1.6 Overall Efficiency and Residual-Conversion Ratio . . . . . . . 79

4.1.7 Application of Residual-Conversion Ratio . . . . . . . . . . . 80

4.2 Fake Leptons . . . . . . . . . . . . . . . . . . . . . . . . . . . . 92

4.2.1 Fake-lepton Backgrounds . . . . . . . . . . . . . . . . . . . 92

4.3 Physics Backgrounds . . . . . . . . . . . . . . . . . . . . 100

4.3 .1 Monte Carlo Scale Factors . . . . . . . . . . . . . . . . 100

4.3 .2 Data Samples . . . . . . . . . . . . . . . . . . . . . . . 101 
4.3 .3 Trigger Efficiency . . . . . . . . . . . . . . . . . . . . . 101

4.3.4 Primary-Vertex Cut Efficiency and Scale Factor . . . . . . . . . 102

4.3.5 Lepton Identification Efficiency and Scale Factor . . . . . . . . . 103

4.3 .6 OSLS Fake Ratio . . . . . . . . . . . . . . . . . . . 103

4.3.7 Low- $E_{T}$ Electron Selection Efficiency and Scale Factor . . . . . 103

4.3.8 High- $E_{T}$ Electron Selection Efficiency and Scale Factor . . . . . 104

4.3.9 Muon Selection Efficiency and Scale Factor . . . . . . . . . . . . 104

4.3.10 Muon Reconstruction Efficiency and Scale Factor . . . . . . . . 104

4.3.11 $Z / \gamma^{*} \rightarrow \ell^{+} \ell^{-}$Cross Section . . . . . . . . . . . . . 104

4.4 Expected Backgrounds ........................ 113

5 Search for the Higgs Boson $\quad \mathbf{1 1 5}$

5.1 Expected Number of Events for the Higgs Boson . . . . . . . . . . . . . 115

5.2 Boosted Decision Trees Discriminant . . . . . . . . . . . . . 116

5.2.1 Decision Tree . . . . . . . . . . . . . . . . . . . . 117

5.2 .2 Boosting Algorithm . . . . . . . . . . . . . . . . . . . . . . 118

5.2 .3 BDT Training Samples . . . . . . . . . . . . . . . . . . . . 120

5.2 .4 BDT Input Variables . . . . . . . . . . . . . . . . . . . . . . . 120

5.2.5 BDT Output and Cross Check . . . . . . . . . . . 126

6 Systematic Uncertainty 143

6.1 Rate Systematic Uncertainty . . . . . . . . . . . . . . . . . . . . . . 143

6.2 Shape Systematic Uncertainty . . . . . . . . . . . . . . . 145

$\begin{array}{lll}7 & \text { Results } & 151\end{array}$

7.1 Likelihood Function . . . . . . . . . . . . . . . . . . . 151

7.2 Upper Limit at a $95 \%$ Confidence Level . . . . . . . . . . . . . . . . . 152

$\begin{array}{llr}8 & \text { Conclusion } & 157\end{array}$

$\begin{array}{lr}\text { A Efficiency and Scale Factor for Event Selection } & 159\end{array}$

A.1 Trigger Efficiency . . . . . . . . . . . . . . . . . . . . . . 159

A.2 Primary-Vertex Cut Efficiency and Scale Factor . . . . . . . . . . . . 163

A.3 Lepton Selection Efficiency and Scale Factor . . . . . . . . . . . . . . . 166

A.3.1 Electron Selection Efficiency and Scale Factor . . . . . . . . . . 166

A.3.2 Muon Selection Efficiency and Scale Factor . . . . . . . . . . . . 173

A.3.3 Muon Reconstruction Efficiency and Scale Factor . . . . . . . . 194

$\begin{array}{ll}\text { B Event Display } & 199\end{array}$ 



\section{List of Figures}

1.1 The potential $V(\phi)$ of the scalar field $\phi$ in the case $\mu^{2}>0$ (left) and

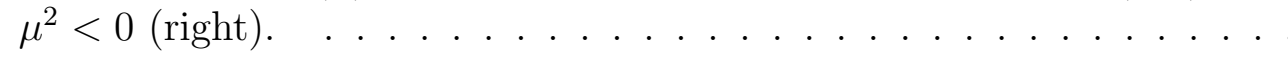

1.2 Feynman diagrams for the Higgs boson production mechanism (from left to right: the gluon-gluon fusion, the associated production with vector boson, the vector boson fusion, and the associated production with heavy quarks). . . . . . . . . . . . . . . .

1.3 Production cross-section of the SM Higgs boson in several processes at the Tevatron. . . . . . . . . . . . . . . . 16

1.4 The branching fraction on the minimal SM Higgs boson (left-side) and the Fermiophobic Higgs boson in 2HDM Type-I (right-side). . . . . . . 18

1.5 Feynman diagrams for the tree-level and one-loop Higgs self-coupling.

1.6 Feynman diagrams for the one-loop contribution of fermion and gauge boson to the Higgs coupling $\lambda . \ldots \ldots \ldots \ldots$

1.7 The triviality (upper) bound and the vacuum stability (lower) bound on the Higgs boson mass as a function of the cut-off scale $\Lambda$ for a top quark mass $M_{t}=175 \pm 6 \mathrm{GeV} / c^{2}$ and $\alpha_{s}\left(M_{Z}\right)=0.118 \pm 0.002 . \quad \ldots . . .20$

1.8 Radiative loop correction for electroweak processes. . . . . . . . . . . . 22

1.9 The $\Delta \chi^{2}$ of the fit to the electroweak precision data as a function of SM Higgs mass. The solid line results by including all data, and the blue band is the estimated theoretical error from missing higher-order corrections. . . . . . . . . . . . . . . . . .

1.10 Confidence Level $\mathrm{CL}_{\mathrm{s}}$ for the signal+background hypothesis in Higgs production at LEP2. The yellow green and yellow band around the median expected line correspond to the $68 \%$ and $95 \%$ probability bands,

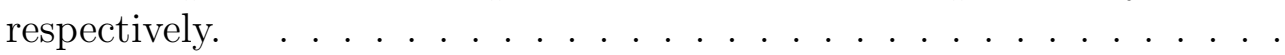

1.11 Combined LEP experiment upper limits for the Higgs bosons decaying into di-photons $B\left(h^{0} \rightarrow \gamma \gamma\right) \times \sigma\left(e^{+} e^{-} \rightarrow h^{0} Z\right) / \sigma(\mathrm{SM})$ as a function of Higgs mass at $95 \%$ confidence level.

1.12 Tevatron combined upper limit on the ratios to the SM Higgs boson production as a function of the Higgs mass at 95\% confidence level. The green and yellow bands indicate the $68 \%$ and $95 \%$ probability regions, respectively. . . . . . . . . . . . . . . . . 
1.13 ATLAS combined upper limit on the ratios to the SM Higgs boson production as a function of the Higgs mass at 95\% confidence level. The green and yellow bands indicate the $68 \%$ and $95 \%$ probability regions, respectively. . . . . . . . . . . . . . . . .

1.14 CMS combined upper limit on the ratios to the SM Higgs boson production as a function of the Higgs mass at 95\% confidence level. The green and yellow bands indicate the $68 \%$ and $95 \%$ probability regions, respectively. . . . . . . . . . . . . . . . . .

2.1 A diagram (left) and aerial photograph (right) of the Fermilab accelerator chain. . . . . . . . . . . . . . . . . 30

2.2 Integrated luminosity as a function of store number between February 2002 and September 2011. . . . . . . . . . . . . . . . . . . . . . . . . . . 33

2.3 Cut away view of the CDF II detector. . . . . . . . . . . . . . . 34

2.4 Elevation view of the CDF II detector. . . . . . . . . . . . . . 35

2.5 End view of Layer 00 (colored), also showing a part of SVX-II (un-colored). 36

$2.63 \mathrm{D}$ view of the three barrels (left) and $r-\phi$ view of the barrel showing the 12 wedges with the 5 layers. . . . . . . . . . . . . 37

2.73 D veiw of the ISL spaceframe. . . . . . . . . . . . . . . . . . . . . 38

$2.8 r-\phi$ veiw (left) and $r-z$ view (right) of the silicon detectors. . . . . 38

2.9 East endplate slots sense and field planes are at the clock-wise edge of each slot. . . . . . . . . . . . . . . . . . . . . . . . . . . . . . . . . . . . . . 49

2.10 Nominal cell layout for SL2. . . . . . . . . . . . . . . . . . . . . . . 40

2.11 Cross section of the plug calorimeter (PEM and PHA) . . . . . . . . 42

2.12 Muon detector coverage in $\eta-\phi$ plane. . . . . . . . . . . . . . . . . . . . . . . . . . . . . . . . 44

2.13 CMX detector in $r-\phi$ plane. . . . . . . . . . . . . . . . . . . 45

2.14 Book diagram of the trigger pass for Level 1 and Level 2. . . . . . . . . 46

2.15 Schamatic diagram of the trigger and DAQ . . . . . . . . . . . . 47

3.1 Normalized distributions of the identification variables for electrons from $Z$ decays compared to those for electron candidates in jet samples (top left: $E / p$, top right: $\mathrm{HAD} / \mathrm{EM}$, bottom left: $\chi_{\text {strip }}^{2}$, bottom right: $\left.E_{\mathrm{CES}} / p^{*}\right)$. 56

3.2 Normalized distributions of the identification variables for electrons from $Z$ decays compared to those for electron candidates in jet samples (top left: $Q \times \Delta x_{\mathrm{CES}}$, top right: $\Delta z_{\mathrm{CES}}$, bottom left: $L_{\mathrm{shr}}$, bottom right:

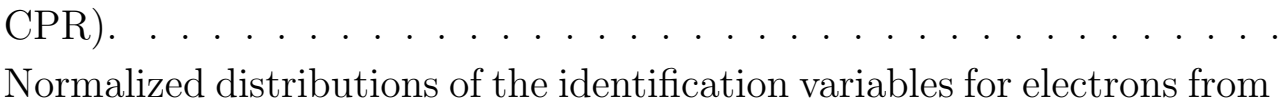

3.3 Normalized distributions of the identification variables for electrons from
$Z$ decays compared to those for electron candidates in jet samples (CP2).
3.4 Normalized distributions of the identification variables for muons from $Z$ decays compared to those for muon candidates in jet samples. . . . 58 
3.5 Normalized distributions of the identification variables for muons from $Z$ decays compared to those for muon candidates in jet samples (top left: $\Delta x_{\mathrm{CMU}}$, top right: $\Delta x_{\mathrm{CMP}}$, bottom: $\left.\Delta x_{\mathrm{CMX}}\right) . \ldots \ldots$

4.1 A theoretical calculation of the relative probability of energy sharing in the photon conversion as a function of fractional electron $\left(e^{-}\right)$en-

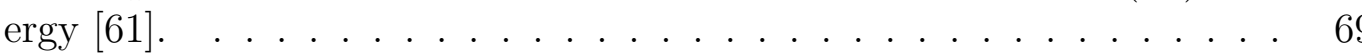

4.2 Distribution of $E / p$ for the seed electron of conversions. . . . . . . . . 71

4.3 Reduced $\chi^{2}$ between the data and the Monte Carlo distributions of reconstructed photon- $p_{T}$ as a function of Monte Carlo slope-parameters. 71

4.4 The reconstructed photon- $p_{T}$ spectrum. . . . . . . . . . . . . 72

4.5 Distribution of the radius of conversion points from the beam line. . . 72

4.6 Conversion-electron $p_{T}$ spectrum. . . . . . . . . . . . . . 73

4.7 Conversion-electron $E_{T}$ spectrum. . . . . . . . . . . . . . . 73

4.8 Partner-track $p_{T}$ spectrum for conversions. The Monte Carlo spectrum is shown at the generator (OBSP) level and fitted to the data in the $p_{T}$ region $2<p_{T}<5 \mathrm{GeV} / c . \ldots \ldots 76$

4.9 Relative conversion-detection efficiency as a function of partner-track $p_{T}$. Only statistical uncertainties are shown. . . . . . . . . . . . 77

4.10 Relative uncertainty of the relative conversion-detection efficiency originating from different sources. . . . . . . . . . . . . .

4.11 Comparison of photon- $p_{T}$ spectra between like-sign lepton + conversion events and Monte Carlo tuned by conversions in inclusive electron data.

4.12 Relative conversion-detection efficiency as a function of seed-electron $E_{T}$ for two cases of slope parameters in MC tuning. . . . . . . . . .

4.13 Relative conversion-detection efficiency as a function of photon $p_{T}$. The uncertainties are statistical only. . . . . . . . . . . . 81

4.14 Comparison of $E / p$ distributions for conversions in like-sign lepton+conversion events, the $\gamma$ Monte Carlo, and the $\pi^{0}$ Monte Carlo. The $p_{T^{-}}$-slope parameters of the Monte Carlo data are tuned by the like-sign lepton+conversion events. The Monte Carlo distributions are normalized to the data. The uncertainties of the real data are statistical only. . .

4.15 Relative uncertainty, shown for different sources, of the relative conversiondetection efficiency as a function of photon $p_{T} . \ldots \ldots 83$

4.16 Illustration of the conversion tagging by means of CES strip information. 83

4.17 Distribution of absolute detector $\eta$ for conversion electrons with the partner track hitting the tower with an $\eta$-index different from the electron.

4.18 Distribution of $z$ position difference on the CES plane between the seed electron and the highest-energy CES cluster in the nearest three towers of each $\phi$ side (right side and wrong side). . . . . . . . . . . . . .

4.19 Distribution of $z$ position difference before the conversion tagging cut (top) and after (bottom). . . . . . . . . . . . . . 
4.20 Conversion-detection efficiency as a function of photon $p_{T}$. The uncertainties are statistical only.

4.21 Residual ratio as a function of photon $p_{T}$. The uncertainties are statistical only.

4.22 Relative uncertainty, shown for different sources, of the residual ratio as a function of photon $p_{T} \ldots \ldots \ldots \ldots \ldots$

4.23 Residual ratio (top) and its relative uncertainties (bottom) as a function of photon $p_{T}$ for the case of our high- $p_{T}$ cut, $E_{T}>20 \mathrm{GeV}$ and $p_{T}>10$ $\mathrm{GeV} / c$. The uncertainties in the top plot are statistical only.

4.24 Fraction of residual conversions with partner-track $p_{T}$ less than $1 \mathrm{GeV} / c$ as a function of photon $p_{T}$ for the case of low lepton- $p_{T}$ cut. . . . . . .

4.25 Fraction of residual conversions with partner-track $p_{T}$ less than $1 \mathrm{GeV} / c$ as a function of photon $p_{T}$ for the case of high lepton- $p_{T}$ cut. . . . . .

4.26 Ratio between the calorimeter isolation and the partner-track $p_{T}$ for conversions. . . . . . . . . . . . . . . . . . 9 91

4.27 Bias due to $\mathrm{W}$ veto in jet samples. . . . . . . . . . . . . . . . . . 94

4.28 Transverse mass distribution. . . . . . . . . . . . . . . . . . 95

$4.29 p_{T}$ distributions in each jet sample. . . . . . . . . . . . . . . . 96

$4.30 p_{T}$ distributions in each jet sample. . . . . . . . . . . . . . . 97

4.31 Fake rates of electrons. The variables are lepton $p_{T}$, pseudorapidity $\eta$, isolation, impact parameter $d_{0}$, the offset angle of one calorimeter $(\mathrm{cph})$, and the difference angle between lepton and missing $E_{T} . \ldots$. . . . .

4.32 Fake rates of muons. The variables are lepton $p_{T}$, pseudorapidity $\eta$, isolation, impact parameter $d_{0}$, the offset angle of one calorimeter (cph), and the difference angle between lepton and missing $E_{T}$. . . . . . . .

4.33 Z $/ \gamma^{*} \rightarrow e^{+} e^{-}$Cross Section to validate the trigger efficiency and scale factors. . . . . . . . . . . . . . . . . . 110

$4.34 Z / \gamma^{*} \rightarrow \mu^{+} \mu^{-}$Cross Section to validate the trigger efficiency and scale factors. The left upper plots is CMUP-CMUP combination, the right upper plots is CMUP-CMX combination, the left lower plots is CMXCMUP combination, and the right upper plots is CMX-CMX combination.111

$4.35 Z / \gamma^{*} \rightarrow \mu^{+} \mu^{-}$Cross Section to validate the trigger efficiency and scale factors. The left upper plots is CMUP-(CMIOCES or CMIOPES) combination, the right upper plots is CMUP-(CMU, CMP, or BMU) combination, the left lower plots is CMX-(CMIOCES or CMIOPES) combination, and the right upper plots is $\mathrm{CMX}-(\mathrm{CMU}, \mathrm{CMP}$, or BMU) combination. . . . . . . . . . . . . . . . . 112

5.1 Schematic view of a decision tree. . . . . . . . . . . . . . . . 117

5.2 1st lepton $E_{T}$, 1st lepton $p_{T}$, and 2nd lepton $p_{T}$ distributions in the signal region (left: linear, right: $\log$ ). . . . . . . . . . . . . . . . 122

5.3 Dilepton mass, dilepton system $p_{T}$, and dilepton system $p_{z}$ in the signal region (left: linear, right: $\log ) \ldots \ldots$. . . . . . . . . . 123 
5.4 Jet multiplicity, missing $E_{T}$, and MetSpec in the signal region (left: linear, right: $\log )$. . . . . . . . . . . . . . . . . . . 124

5.5 $H_{T}$, Sphericity, and Aplanarity in the signal region (left: linear, right: $\log ) \ldots \ldots \ldots \ldots \ldots \ldots \ldots$

5.6 1st lepton $E_{T}$, 1st lepton $p_{T}$, and 2nd lepton $p_{T}$ distributions in the lepton ID side-band region (left: linear, right: log). . . . . . . . . . . . 127

5.7 Dilepton mass, dilepton system $p_{T}$, and dilepton system $p_{z}$ in the lepton ID side-band region (left: linear, right: $\log$ ). . . . . . . . . . . . . 128

5.8 Jet multiplicity, missing $E_{T}$, and MetSpec in the lepton ID side-band region (left: linear, right: $\log ) \ldots \ldots \ldots \ldots$

$5.9 H_{T}$, Sphericity, and Aplanarity in the lepton ID side-band region (left: linear, right: $\log ) \ldots \ldots \ldots$. . . . . . . . . . . . . . 130

5.101 st lepton $E_{T}$, 1st lepton $p_{T}$, and 2nd lepton $p_{T}$ distributions in the zero-silicon region (left: linear, right: $\log$ ).

5.11 Dilepton mass, dilepton system $p_{T}$, and dilepton system $p_{z}$ in the zerosilicon region (left: linear, right: log).

5.12 Jet multiplicity, missing $E_{T}$, and MetSpec in the zero-silicon region (left: linear, right: $\log ) \ldots \ldots \ldots \ldots \ldots$

$5.13 H_{T}$, Sphericity, and Aplanarity in the zero-silicon region (left: linear, right: $\log ) \ldots \ldots \ldots \ldots \ldots \ldots$

5.14 1st lepton $E_{T}$, 1st lepton $p_{T}$, and 2nd lepton $p_{T}$ distributions in the opposite-sign region (left: linear, right: $\log$ ). . . . . . . . . . . . . 135

5.15 Dilepton mass, dilepton system $p_{T}$, and dilepton system $p_{z}$ in the oppositesign region (left: linear, right: log).

5.16 Jet multiplicity, missing $E_{T}$, and MetSpec in the opposite-sign region (left: linear, right: $\log ) \ldots \ldots \ldots$. . . . . . . . . . 137

$5.17 H_{T}$, Sphericity, and Aplanarity in the opposite-sign region (left: linear, right: $\log ) \ldots \ldots \ldots \ldots \ldots$

5.18 BDT output on $m_{H}=110,160\left(\mathrm{GeV} / c^{2}\right)$ in the side-band region (left: linear, right: $\log ) \ldots \ldots \ldots \ldots \ldots$

5.19 BDT output on $m_{H}=110,160\left(\mathrm{GeV} / c^{2}\right)$ in the zero-silicon region (left: linear, right: $\log ) \ldots \ldots \ldots$. . . . . . . . . . . . 140

5.20 BDT output on $m_{H}=110,160\left(\mathrm{GeV} / c^{2}\right)$ in the OS region (left: linear, right: $\log ) \ldots \ldots \ldots \ldots \ldots 14 \ldots \ldots \ldots$

5.21 BDT output on $m_{H}=110,160\left(\mathrm{GeV} / c^{2}\right)$ in the signal region (left: linear, right: $\log ) \ldots \ldots \ldots \ldots$. . . . . . . . . . . . . . 142

6.1 Shape systematic uncertainties due to jet energy scale for backgrounds trained on $m_{H}=110 \mathrm{GeV} / c^{2}$. (Fake\&ResCo means fake lepton and residual-photon conversion backgrounds.) . . . . . . . . . . . . . . 146

6.2 Shape systematic uncertainties due to jet energy scale for Higgs Mass

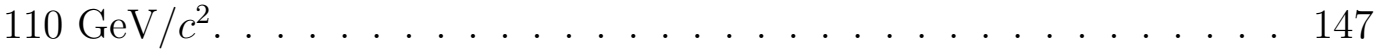


6.3 Shape systematic uncertainties due to jet energy scale for backgrounds trained on $m_{H}=160 \mathrm{GeV} / c^{2}$. (Fake\&ResCo means fake lepton and residual-photon conversion backgrounds.) . . . . . . . . . . . . . . . 148

6.4 Shape systematic uncertainties due to jet energy scale for Higgs Mass $160 \mathrm{GeV} / c^{2} \ldots \ldots \ldots \ldots \ldots \ldots$

7.1 Limits on the production cross-section times branching fraction $\sigma(p \bar{p} \rightarrow V h) \times$ $B_{F}\left(h \rightarrow W^{*+} W^{*-}\right)$ to fermiophobic higgs Prediction at the $95 \%$ C.L.

7.2 Limits on the production cross-section times branching fraction $\sigma(p \bar{p} \rightarrow V h) \times$ $B_{F}\left(h \rightarrow W^{*+} W^{*-}\right)$ to the Standard Model higgs Prediction at the $95 \%$ C.L. . . . . . . . . . . . . . . . . . . . 156

B.1 Event Display of the $W Z$ candidate event with BDT Output $=0.89$ trained by the Higgs boson mass of $110 \mathrm{GeV} / c^{2}$. (top left: COT, top right: Calorimeter, bottom left: SVX, bottom right: Calorimter $\left.E_{T}\right)$. . . . . 199

B.2 Event Display of the fake candidate event with BDT Output $=-0.90$ trained by the Higgs boson mass of $110 \mathrm{GeV} / c^{2}$. (top left: COT, top right: Calorimeter, bottom left: SVX, bottom right: Calorimter $E_{T}$ ).

B.3 Event Display of the $W Z$ Monte Carlo simulation with BDT Ouftput $=0.92$ trained by the Higgs boson mass of $110 \mathrm{GeV} / c^{2}$. (top left: COT, top right: Calorimeter, bottom left: SVX, bottom right: Calorimter $\left.E_{T}\right) \ldots \ldots \ldots \ldots \ldots \ldots$

B.4 Event Display of the Higgs Monte Carlo simulation $\left(m_{H}=110 \mathrm{GeV} / c^{2}\right)$ with BDT Ouftput $=0.92$ trained by the Higgs boson mass of $110 \mathrm{GeV} / c^{2}$. (top left: COT, top right: Calorimeter, bottom left: SVX, bottom right: Calorimter $E_{T}$ ) . . . . . . . . . . . . . . 202 


\section{List of Tables}

1.1 List of the leptons and quarks and its properties in the Standard Model [1]. 3

1.2 Summry of the forces and gauge bosons in the Standard Model. . . . . 3

1.3 The neutral Higgs boson couplings to gauge bosons $\left(V=W^{ \pm}, Z\right)$ and fermions in 2HDM Type-I. . . . . . . . . . . . . . . . . . 14

2.1 Accelerator parameters for Run II configurations. . . . . . . . . . . . . 32

2.2 Design parameters of the Silicon Vertex Detector. . . . . . . . . . . . . . 37

2.3 Design parameters of the Central Outer Tracker. . . . . . . . . . . . . . 39

2.4 Design parameters of the calorimeter. . . . . . . . . . . . . . . . . . . . . . . . . . 43

2.5 Design parameters of the moun detector. . . . . . . . . . . . . . . 44

3.1 List of the datasets. . . . . . . . . . . . . . . . . . 61

3.2 Main features of the ELECTRON_CENTRAL_18_V* triggers. . . . . . . . . . 62

3.3 Main features of the CMUP_MUON18_* triggers. . . . . . . . . . . . . . . 62

3.4 Main features of the CMX_MUON18_* triggers. . . . . . . . . . . . . . . 63

3.5 Event pre-selection and lepton selection cuts. . . . . . . . . . . . . 64

3.6 Event pre-selection and lepton selection cuts. . . . . . . . . . . . . 64

3.7 Dilepton selection cuts. . . . . . . . . . . . . . . . . . . 64

3.8 Physics objects used to identify and remove $Z$ bosons. . . . . . . . . 65

4.1 Monte Carlo samples. . . . . . . . . . . . . . . . . . . . 69

4.2 Event pre-selection and the denominator track selection. . . . . . . . . 95

4.3 Summary of the Monte Carlo samples. . . . . . . . . . . . . . . . . . . 101

4.4 The number of $Z / \gamma^{*}\left(66<M_{\ell \ell}<116 \mathrm{GeV}\right)$ event and the cross section for CEM-CEM pair. (Note : Quote NNLO cross section $(\sigma=251.3 \pm 5$ $\mathrm{pb})$ to estimate the significance for cross section.) . . . . . . . . 106

4.5 The number of $Z / \gamma^{*}\left(66<M_{\ell \ell}<116 \mathrm{GeV}\right)$ event and the cross section for CMUP-CMUP pair. (Note : Quote NNLO cross section $(\sigma=251.3$ $\pm 5 \mathrm{pb})$ to estimate the significance for cross section.) . . . . . . 106

4.6 The number of $Z / \gamma^{*}\left(66<M_{\ell \ell}<116 \mathrm{GeV}\right)$ event and the cross section for CMX-CMUP pair. (Note : Quote NNLO cross section $(\sigma=251.3 \pm$ $5 \mathrm{pb})$ to estimate the significance for cross section.) . . . . . . . . . 106 
4.7 The number of $Z / \gamma^{*}\left(66<M_{\ell \ell}<116 \mathrm{GeV}\right)$ event and the cross section for CMUP-CMX pair. (Note: Quote NNLO cross section $(\sigma=251.3 \pm$ $5 \mathrm{pb})$ to estimate the significance for cross section.) . . . . . . . . 107

4.8 The number of $Z / \gamma^{*}\left(66<M_{\ell \ell}<116 \mathrm{GeV}\right)$ event and the cross section for CMX-CMX pair. (Note : Quote NNLO cross section $(\sigma=251.3 \pm$ $5 \mathrm{pb})$ to estimate the significance for cross section.) . . . . . . . . . 107

4.9 The number of $Z / \gamma^{*}\left(66<M_{\ell \ell}<116 \mathrm{GeV}\right)$ event and the cross section for CMUP-(CMIOCES or CMIOPES) pair. (Note : Quote NNLO cross section ( $\sigma=251.3 \pm 5 \mathrm{pb})$ to estimate the significance for cross section.) 107

4.10 The number of $Z / \gamma^{*}\left(66<M_{\ell \ell}<116 \mathrm{GeV}\right)$ event and the cross section for CMX-(CMIOCES or CMIOPES) pair. (Note : Quote NNLO cross section $(\sigma=251.3 \pm 5 \mathrm{pb})$ to estimate the significance for cross section.) 108

4.11 The number of $Z / \gamma^{*}\left(66<M_{\ell \ell}<116 \mathrm{GeV}\right)$ event and the cross section for CMUP-(CMU, CMP, or BMU) pair. (Note : Quote NNLO cross section $(\sigma=251.3 \pm 5 \mathrm{pb})$ to estimate the significance for cross section.) 108

4.12 The number of $Z / \gamma^{*}\left(66<M_{\ell \ell}<116 \mathrm{GeV}\right)$ event and the cross section for CMX-(CMU, CMP, or BMU) pair. (Note : Quote NNLO cross section $(\sigma=251.3 \pm 5 \mathrm{pb})$ to estimate the significance for cross section.) 108

4.13 Summary of observed events, expected events, and Significance of $Z \rightarrow \ell^{ \pm} \ell^{\mp}$ pairs in period 0 through 38. Note: CMIO means CMIO-CES or CMIOPES, OM means CMU, CMP, or BMU. . . . . . . . . . . . . . . 109

4.14 Background expectation and observed number of events for side-band, zero-silicon, opposite-sign and like-sign(signal) dilepton events. . . . . 114

5.1 Theoretical cross sections and branching fractions on each mass of the Higgs boson. . . . . . . . . . . . . . . . . 116

5.2 Expected number of events for signal passing baseline event selections (FP: Fermiophobic Higgs). . . . . . . . . . . . . . . . . . . . 116

6.1 Systematic uncertainties for the $W h$ Monte Carlo samples. . . . . . . . 144

6.2 Systematic uncertainties for the $Z h$ Monte Carlo samples. . . . . . . . . 145

6.3 Systematic uncertainties for backgrounds. . . . . . . . . . . . . 145

7.1 The $95 \%$ C.L. limit on the fermiophobic higgs. Including both $W h$ and Zh signals. . . . . . . . . . . . . . . . . . . . 154

7.2 The $95 \%$ C.L. limit on the Standard Model higgs. Including both Wh and $Z h$ signals. . . . . . . . . . . . . . . . . . 154

A.1 ELECTRON_CENTRAL_18 trigger efficiency for each period. . . . . . . . . . 160

A.2 MUON_CMUP18 trigger efficiency for each period. . . . . . . . . . . . . 161

A.3 MUON_CMX18 trigger efficiency for each period. . . . . . . . . . . . 162

A.4 Primary vertex cut efficiency and scale factor for od data set. . . . . . 163

A.5 Primary vertex cut efficiency and scale factor for $\mathrm{Oh}$ data set. . . . . . 163

A.6 Primary vertex cut efficiency and scale factor for $0 i$ data set. . . . . . 163 
A.7 Primary vertex cut efficiency and scale factor for $0 \mathrm{j}$ data set. . . . . . 164

A.8 Primary vertex cut efficiency and scale factor for 0k data set. . . . . . 164

A.9 Primary vertex cut efficiency and scale factor for 0m data set. . . . . . 164

A.10 Primary vertex cut efficiency and scale factor for 0p data set. . . . . . 165

A.11 Low $E_{T}$ electron selection cut efficiencies and scale factors for Od data set.

A.12 High $E_{T}$ electron selection cut efficiencies and scale factors for od data set. . . . . . . . . . . . . . . . .

A.13 Low $E_{T}$ electron selection cut efficiencies and scale factors for $0 \mathrm{~h}$ data set.

A.14 High $E_{T}$ electron selection cut efficiencies and scale factors for $0 \mathrm{~h}$ data set. . . . . . . . . . . . . . . .

A.15 Low $E_{T}$ electron selection cut efficiencies and scale factors for $0 i$ data set.

A.16 High $E_{T}$ electron selection cut efficiencies and scale factors for $0 \mathrm{~h}$ data set. . . . . . . . . . . . . . . . .

A.17 Low $E_{T}$ electron selection cut efficiencies and scale factors for $0 \mathrm{j}$ data set.

A.18 High $E_{T}$ electron selection cut efficiencies and scale factors for $0 \mathrm{j}$ data set.

A.19 Low $E_{T}$ electron selection cut efficiencies and scale factors for $0 \mathrm{k}$ data set.

A.20 High $E_{T}$ electron selection cut efficiencies and scale factors for 0k data set.

A.21 Low $E_{T}$ electron selection cut efficiencies and scale factors for 0m data set.

A.22 High $E_{T}$ electron selection cut efficiencies and scale factors for 0m data set.

A.23 Low $E_{T}$ electron selection cut efficiencies and scale factors for $0 \mathrm{p}$ data set.

A.24 High $E_{T}$ electron selection cut efficiencies and scale factors for $0 \mathrm{p}$ data set.

A.25 Muon selection cut efficiencies and scale factors from CMUP-CMUP for Od data set.

A.26 Muon selection cut efficiencies and scale factors from CMX-CMUP for Od data set.

A.27 Muon selection cut efficiencies and scale factors from CMUP-CMX for od data set.

A.28 Muon selection cut efficiencies and scale factors from CMX-CMX for 0d data set.

A.29 Muon selection cut efficiencies and scale factors from CMUP-(CMIOCES or CMIOPES) for od data set. 
A.30 Muon selection cut efficiencies and scale factors from CMX-(CMIOCES or CMIOPES) for Od data set. . . . . . . . . . . . . . . 175

A.31 Muon selection cut efficiencies and scale factors from CMUP-(CMU, CMP, or BMU) for od data set. . . . . . . . . . . . . . 175

A.32 Muon selection cut efficiencies and scale factors from CMX-(CMU, CMP, or BMU) for Od data set. . . . . . . . . . . . . . . .

A.33 Muon selection cut efficiencies and scale factors from CMUP-CMUP for Oh data set.

A.34 Muon selection cut efficiencies and scale factors from CMX-CMUP for Oh data set.

A.35 Muon selection cut efficiencies and scale factors from CMUP-CMX for Oh data set.

A.36 Muon selection cut efficiencies and scale factors from CMX-CMX for $\mathrm{Oh}$ data set.

A.37 Muon selection cut efficiencies and scale factors from CMUP-(CMIOCES or CMIOPES) for Oh data set.

A.38 Muon selection cut efficiencies and scale factors from CMX-(CMIOCES or CMIOPES) for Oh data set.

A.39 Muon selection cut efficiencies and scale factors from CMUP-(CMU, $\mathrm{CMP}$, or BMU) for $\mathrm{Oh}$ data set. . . . . . . . . . . . . . . .

A.40 Muon selection cut efficiencies and scale factors from CMX-(CMU, CMP, or BMU) for Oh data set.

A.41 Muon selection cut efficiencies and scale factors from CMUP-CMUP for

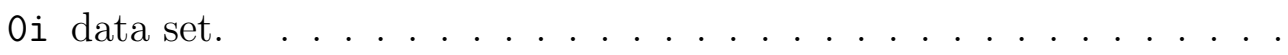

A.42 Muon selection cut efficiencies and scale factors from CMX-CMUP for 0 i data set.

A.43 Muon selection cut efficiencies and scale factors from CMUP-CMX for Oi data set.

A.44 Muon selection cut efficiencies and scale factors from CMX-CMX for $0 i$ data set.

A.45 Muon selection cut efficiencies and scale factors from CMUP-(CMIOCES or CMIOPES) for 0i data set.

A.46 Muon selection cut efficiencies and scale factors from CMX-(CMIOCES or CMIOPES) for 0i data set.

A.47 Muon selection cut efficiencies and scale factors from CMUP-(CMU, CMP, or BMU) for $0 i$ data set.

A.48 Muon selection cut efficiencies and scale factors from CMX-(CMU, CMP, or BMU) for 0i data set.

A.49 Muon selection cut efficiencies and scale factors from CMUP-CMUP for $0 \mathrm{j}$ data set.

A.50 Muon selection cut efficiencies and scale factors from CMX-CMUP for $0 j$ data set. 
A.51 Muon selection cut efficiencies and scale factors from CMUP-CMX for

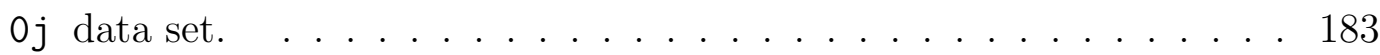

A.52 Muon selection cut efficiencies and scale factors from CMX-CMX for $0 j$ data set.

A.53 Muon selection cut efficiencies and scale factors from CMUP-(CMIOCES or CMIOPES) for $0 \mathrm{j}$ data set. . . . . . . . . . . . . . 183

A.54 Muon selection cut efficiencies and scale factors from CMX-(CMIOCES or CMIOPES) for $0 \mathrm{j}$ data set. . . . . . . . . . . . . . . . 184

A.55 Muon selection cut efficiencies and scale factors from CMUP-(CMU, CMP, or BMU) for $0 j$ data set.

A.56 Muon selection cut efficiencies and scale factors from CMX-(CMU, CMP, or BMU) for $0 \mathrm{j}$ data set. . . . . . . . . . . . . . . . . . . . 184

A.57 Muon selection cut efficiencies and scale factors from CMUP-CMUP for Ok data set.

A.58 Muon selection cut efficiencies and scale factors from CMX-CMUP for ok data set.

A.59 Muon selection cut efficiencies and scale factors from CMUP-CMX for Ok data set.

A.60 Muon selection cut efficiencies and scale factors from CMX-CMX for 0k data set.

A.61 Muon selection cut efficiencies and scale factors from CMUP-(CMIOCES or CMIOPES) for Ok data set.

A.62 Muon selection cut efficiencies and scale factors from CMX-(CMIOCES or CMIOPES) for $0 \mathrm{k}$ data set. . . . . . . . . . . . . . 187

A.63 Muon selection cut efficiencies and scale factors from CMUP-(CMU, CMP, or BMU) for 0k data set. . . . . . . . . . . . . . 187

A.64 Muon selection cut efficiencies and scale factors from CMX-(CMU, CMP, or BMU) for $0 \mathrm{k}$ data set. . . . . . . . . . . . . . . .

A.65 Muon selection cut efficiencies and scale factors from CMUP-CMUP for Om data set.

A.66 Muon selection cut efficiencies and scale factors from CMX-CMUP for Om data set.

A.67 Muon selection cut efficiencies and scale factors from CMUP-CMX for Om data set.

A.68 Muon selection cut efficiencies and scale factors from CMX-CMX for 0m data set.

A.69 Muon selection cut efficiencies and scale factors from CMUP-(CMIOCES or CMIOPES) for Om data set.

A.70 Muon selection cut efficiencies and scale factors from CMX-(CMIOCES or CMIOPES) for Om data set.

A.71 Muon selection cut efficiencies and scale factors from CMUP-(CMU, CMP, or BMU) for 0m data set. 
A.72 Muon selection cut efficiencies and scale factors from CMX-(CMU, CMP, or BMU) for $0 \mathrm{~m}$ data set. . . . . . . . . . . . . . . . . . 190

A.73 Muon selection cut efficiencies and scale factors from CMUP-CMUP for Op data set.

A.74 Muon selection cut efficiencies and scale factors from CMX-CMUP for Op data set.

A.75 Muon selection cut efficiencies and scale factors from CMUP-CMX for Op data set.

A.76 Muon selection cut efficiencies and scale factors from CMX-CMX for 0p data set.

A.77 Muon selection cut efficiencies and scale factors from CMUP-(CMIOCES or CMIOPES) for Op data set. . . . . . . . . . . . . . . . . . 192

A.78 Muon selection cut efficiencies and scale factors from CMX-(CMIOCES or CMIOPES) for Op data set.

A.79 Muon selection cut efficiencies and scale factors from CMUP-(CMU, CMP, or BMU) for Op data set.

A.80 Muon selection cut efficiencies and scale factors from CMX-(CMU, CMP, or BMU) for $0 p$ data set.

A.81 Muon reconstruction efficiencies and scale factors in CMUP-triggered events for Od data set. (AR : Arch, MS : Miniskirt, KS : Keystone) . . 194

A.82 Muon reconstruction efficiencies and scale factors in CMX-triggered events for Od data set. (AR : Arch, MS : Miniskirt, KS : Keystone) . . . . . .

A.83 Muon reconstruction efficiencies and scale factors in CMUP-triggered events for Oh data set. (AR : Arch, MS : Miniskirt, KS : Keystone) . . 194

A.84 Muon reconstruction efficiencies and scale factors in CMX-triggered events for Oh data set. (AR : Arch, MS : Miniskirt, KS : Keystone) . . . . . . 195

A.85 Muon reconstruction efficiencies and scale factors in CMUP-triggered events for 0i data set. (AR : Arch, MS : Miniskirt, KS : Keystone) . . 195

A.86 Muon reconstruction efficiencies and scale factors in CMX-triggered events for 0i data set. (AR : Arch, MS : Miniskirt, KS : Keystone) . . . . . . 195

A.87 Muon reconstruction efficiencies and scale factors in CMUP-triggered events for $0 \mathrm{j}$ data set. (AR : Arch, MS : Miniskirt, KS : Keystone) . . 196

A.88 Muon reconstruction efficiencies and scale factors in CMX-triggered events for $0 \mathrm{j}$ data set. (AR : Arch, MS : Miniskirt, KS : Keystone) . . . . . . 196

A.89 Muon reconstruction efficiencies and scale factors in CMUP-triggered events for 0k data set. (AR : Arch, MS : Miniskirt, KS : Keystone) . . 196

A.90 Muon reconstruction efficiencies and scale factors in CMX-triggered events for Ok data set. (AR : Arch, MS : Miniskirt, KS : Keystone) . . . . . . 197

A.91 Muon reconstruction efficiencies and scale factors in CMUP-triggered events for Om data set. (AR : Arch, MS : Miniskirt, KS : Keystone) . . 197

A.92 Muon reconstruction efficiencies and scale factors in CMX-triggered events for Om data set. (AR : Arch, MS : Miniskirt, KS : Keystone) . . . . . . 
A.93 Muon reconstruction efficiencies and scale factors in CMUP-triggered events for 0p data set. (AR : Arch, MS : Miniskirt, KS : Keystone) . . 198

A.94 Muon reconstruction efficiencies and scale factors in CMX-triggered events for Op data set. (AR : Arch, MS : Miniskirt, KS : Keystone) . . . . 198 



\section{Chapter 1}

\section{Introduction}

People have been tried to understand the nature. Here, the nature means not only around people on the earth, but also extremely large or small scales. Some physicists study the nature in the large scale, that is, the universe. Other physicists, particle physicists, investigate the small scale by colliding high-energy particles to look at the internal structures and their dynamics. In both sides, the goal is the same: "to understand the beginning of the universe."

In particle physics, the standard model has been explained many results from various experiments. However, in the standard model, the Higgs boson has not been discovered yet.

In this section, the standard model, the Higgs boson and current constraints from various experiments are explained.

\subsection{The Standard Model}

The standard model (SM) is one of the theories in particle physics based on the gauge field theory which is invariant under the gauge transformation. The SM describes the phenomena and properties of the elementary particles which have been tested by various experiments. The SM consists of three gauge field theories: "quantum electrodynamics (QED)", "quantum chromodynamics (QCD)" and "weak theory". The QED is based on local $U(1)$ symmetry, and describes the electromagnetic interaction between charged particles. The QCD describes the strong interaction between quarks and gluons based on the local $S U(3)_{C}$ symmetry. The weak theory describes weak interactions in the nuclei based on the local $S U(2)$ symmetry. The QED and the weak theory are unified in the SM framework as $S U(2)_{L} \otimes U(1)_{Y}$ gauge theory. In addition, the "Higgs mechanism" plays a key roll to give a "Mass" to a particle with keeping the gauge invariance in the theory. If the Higgs mechanism is occured, at least one additional particle is expected, which is called Higgs boson. The Higgs boson is not discovered yet. 


\subsubsection{Elementary Particles in the Standard Model}

In the SM, there are mainly 2 types elementary particles, so-called "Fermion" and "Boson", respectively. The Fermions construct matters in the universe, while the Bosons mediate forces between the elementary particles. The visible complex matters in this world are made up of them. This following section describes the elementary particles in some detail.

\section{Fermion}

A particle called Fermion obeys the "Pauli Exclusion Principle", i.e. it has half-integral spin. In the SM, the Fermions are classified into six leptons and six quarks. The three of the six leptons are charged lepton, which are "electron", "muon", and "tau", they have different mass, respectively, however its spin, weak isospin, and electric charge are same. The remaining three leptons have no electric charge so-called "neutrino", they have a lepton flavor (lepton number) same as corresponding charged lepton, when electron has +1 electron number, the corresponding neutrino so-called "electron neutrino" has +1 electron number.

The three of the six quarks have $2 / 3$ electric charge, so-called "up", "charm", and "top" quark respectively, they are collectively called "up-type quark" while the other three quarks is $-1 / 3$ electric charge called "down", "strange", and "bottom", collectively "down-type quark". They also have color charge which is source of the strong interaction.

In addition, there are antiparticle for each fermion, which have opposite quantum numbers corresponding to the each fermion. Table 1.1 shows the list of the leptons and the quarks.

\section{$\underline{\text { Boson }}$}

The Bosons play a role in mediating force between the elementary particles corresponding to type of forces. Such bosons are especially called "gauge boson". In the present, it is believed that there are at least 4 kind of force, "Electromagnetic", "Weak", "Strong", and "Gravity", however the Gravity force is excluded in the SM due to normalization problem, and its extremely small affect in the particle world. The electromagnetic forces are propagated via "photon" by feeling electric charge which is gauge boson in the electromagnetic field. The photon has spin 1, and massless means that the force caries to infinity. The weak force interactions are mediated by $W^{ \pm}$and $Z^{0}$ bosons have 80 and $91 \mathrm{GeV} / c^{2}$ mass, respectively, unlike electromagnetic force, it can effect within short range $\left(\sim 10^{-16} \mathrm{~cm}\right)$. The strong force interactions are occurred by exchanging gauge boson so-called "gluon" via color charge, which can propagate within finite range due to "asymptotic freedom". The color charge are conventionally expressed as 3 colors, "red $(r)$ ", "blue $(b)$ ", and "green $(g)$ ", which is introduced by taking Pauli Exclusion Principle in Hadrons (Baryons and Mesons) into account, for example $\Delta^{++}$ 
particle is constructed by 3 up-quarks, it can be expressed as $\left(u_{r}, u_{b}, u_{g}\right)$. The force mediating particles, i.e. gauge bosons, are shown in Table 1.2 [1].

\begin{tabular}{lcccccc}
\hline \hline Name & Symbol & \multicolumn{2}{c}{ Mass } & $\begin{array}{c}\text { Charge } \\
(Q /|e|)\end{array}$ & Spin & Weak Isospin \\
& \multicolumn{6}{c}{ Leptons } \\
\hline electron & $e$ & 0.5109 & $\mathrm{MeV} / c^{2}$ & -1 & $1 / 2$ & $+1 / 2$ \\
electron neutrino & $\nu_{e}$ & $<225(95 \% \mathrm{CL})$ & $\mathrm{eV} / c^{2}$ & 0 & $1 / 2$ & $-1 / 2$ \\
muon & $\mu$ & 105.7 & $\mathrm{MeV} / c^{2}$ & -1 & $1 / 2$ & $+1 / 2$ \\
muon neutrino & $\nu_{\mu}$ & $<0.19(90 \% \mathrm{CL})$ & $\mathrm{MeV} / c^{2}$ & 0 & $1 / 2$ & $-1 / 2$ \\
tau & $\tau$ & 1776.8 & $\mathrm{MeV} / c^{2}$ & -1 & $1 / 2$ & $+1 / 2$ \\
tau neutrino & $\nu_{\tau}$ & $<18.2(95 \% \mathrm{CL})$ & $\mathrm{MeV} / c^{2}$ & 0 & $1 / 2$ & $-1 / 2$ \\
\hline \multicolumn{7}{c}{$\mathrm{Quarks}$} \\
up & $u$ & $2.55_{-1.05}^{+0.75}$ & $\mathrm{MeV} / c^{2}$ & $+2 / 3$ & $1 / 2$ & $+1 / 2$ \\
down & $d$ & $5.04_{-1.54}^{+0.96}$ & $\mathrm{MeV} / c^{2}$ & $-1 / 3$ & $1 / 2$ & $-1 / 2$ \\
charm & $c$ & $1.27_{-0.11}^{+0.07}$ & $\mathrm{GeV} / c^{2}$ & $+2 / 3$ & $1 / 2$ & $+1 / 2$ \\
strange & $s$ & $104_{-34}^{+26}$ & $\mathrm{MeV} / c^{2}$ & $-1 / 3$ & $1 / 2$ & $-1 / 2$ \\
top & $t$ & $171.2 \pm 2.1$ & $\mathrm{GeV} / c^{2}$ & $+2 / 3$ & $1 / 2$ & $+1 / 2$ \\
bottm & $b$ & $4.20_{-0.07}^{+0.17}$ & $\mathrm{GeV} / c^{2}$ & $-1 / 3$ & $1 / 2$ & $-1 / 2$ \\
\hline \hline
\end{tabular}

Table 1.1: List of the leptons and quarks and its properties in the Standard Model [1].

\begin{tabular}{lccccc}
\hline \hline Interaction & $\begin{array}{c}\text { Gauge boson } \\
(\text { symbol })\end{array}$ & $\begin{array}{c}\text { Mass } \\
\left(\mathrm{GeV} / \mathrm{c}^{2}\right)\end{array}$ & $\begin{array}{c}\text { Effective } \\
\text { coupling }\end{array}$ & $\begin{array}{c}\text { Range } \\
{[\mathrm{cm}]}\end{array}$ & $\begin{array}{c}\text { Typical time } \\
{[\mathrm{s}]}\end{array}$ \\
\hline Electromagnetic & photon $(\gamma)$ & 0 & $1 / 137$ & $\infty$ & $10^{-20}$ \\
Weak & $W^{ \pm}, Z^{0}$ & $80.4,91.2$ & $10^{-5}$ & $10^{-16}$ & $10^{-10}$ \\
Strong & gluon $(g)$ & 0 & $\sim 1$ & $10^{-13}$ & $10^{-23}$ \\
\hline \hline
\end{tabular}

Table 1.2: Summry of the forces and gauge bosons in the Standard Model.

\subsubsection{Quantum Electrodynamics: $U(1)$}

Quantum Electrodynamics (QED) is relativistic quantum field theory of the classical electromagnetism [2]. QED has the structure of an Abelian gauge theory with a $U(1)$ gauge group. The gauge field, which mediates the interaction between the charged $1 / 2$ spin fields, is the electromagnetic field. An electron is described by a complex field and the Lagrangian is written as follows,

$$
\mathscr{L}=i \bar{\psi} \gamma_{\mu} \partial^{\mu} \psi-m \psi \bar{\psi}
$$


The Lagrangian is invariant under the phase transformation,

$$
\psi \rightarrow e^{i \alpha} \psi
$$

where $\alpha$ is a real constant. The family of phase transformations $U(\alpha) \equiv e^{i \alpha}$ forms a unitary Abelian group known as the $U(1)$ group. Using Neother's theorem, this invariant implies the existence of a conserved current and charge,

$$
\partial_{\mu} j^{\mu}=0, j^{\mu}=-e \bar{\psi} \gamma^{\mu} \psi, Q=\int d^{3} x j^{0}
$$

In addition, the local gauge transformation is generalized as

$$
\psi \rightarrow e^{i \alpha(x)} \psi
$$

where $\alpha(x)$ depends on space and time in a completely arbitrary way. Now, the Lagrangian (1.1) is not invariant under such phase transformation. Using (1.4),

$$
\bar{\psi} \rightarrow e^{-i \alpha(x)} \bar{\psi}
$$

the last term of the Lagrangian is invariant, however the term of derivative $\psi$ is not as follows,

$$
\partial_{\mu} \psi \rightarrow e^{i \alpha(x)} \partial_{\mu} \psi+i e^{i \alpha(x)} \psi \partial_{\mu} \alpha
$$

and the $\partial_{\mu} \alpha$ term breaks the invariant of the Lagrangian. To impose invariance of the Lagrangian under local gauge transformation, the derivative $\partial_{\mu}$ is modified as $D_{\mu}$, the treatment covariantly transforms the Lagrangian under the phase transformation,

$$
\begin{aligned}
D_{\mu} \psi & \rightarrow e^{i \alpha(x)} D_{\mu} \psi, \\
D_{\mu} & \equiv \partial_{\mu}-i e A_{\mu},
\end{aligned}
$$

where a vector field $A_{\mu}$ is introduced to cancel the unwanted term in (1.6), and the vector field transforms as,

$$
A_{\mu} \rightarrow A_{\mu}+\frac{1}{e} \partial_{\mu} \alpha
$$

Invariance of the Lagrangian (1.1) under the local gauge transformation (1.4) is achieved by replacing $\partial_{\mu}$ by $D_{\mu}$,

$$
\begin{aligned}
\mathscr{L} & =i \bar{\psi} \gamma_{\mu} D^{\mu} \psi-m \psi \bar{\psi} \\
& =\bar{\psi}\left(i \gamma^{\mu} \partial_{\mu}-m\right) \psi+e \bar{\psi} \gamma^{\mu} \psi A_{\mu} .
\end{aligned}
$$

By demanding local phase invariance, it forces to introduce a vector field $A_{\mu}$, i.e. gauge field in QED. If the additional field is regarded as the physical photon field, the Lagrangian is added a term corresponding to its kinetic energy. Since the kinetic term 
must be invariant under (1.9), it can only involve the gauge invariant field strength tensor

$$
F_{\mu \nu}=\partial_{\mu} A_{\nu}-\partial_{\nu} A_{\mu}
$$

Finally, the Lagrangian of QED is expressed as follows,

$$
\mathscr{L}=\bar{\psi}\left(i \gamma^{\mu} \partial_{\mu}-m\right) \psi+e \bar{\psi} \gamma^{\mu} \psi A_{\mu}-\frac{1}{4} F_{\mu \nu} F^{\mu \nu}
$$

The addition of a mass term $(1 / 2) m^{2} A_{\mu} A^{\mu}$ is prohibited by gauge invariance. The gauge particle must be massless and the gauge field can propagate to an infinite range.

\subsubsection{Quantum Chromodynamics: $S U(3)_{C}$}

Quantum Chromodynamics (QCD) is the gauge theory for strong interactions [2]. QCD is based on the extension of the QED idea, however it has a gauge transformation invariant under $S U(3)$ group on quark color fields. The Lagrangian is written in the following,

$$
\mathscr{L}=\bar{q}_{j}\left(i \gamma^{\mu} \partial_{\mu}-m\right) q_{j}
$$

where $q_{j}(j=1,2,3)$ denotes the three color fields. The Lagrangian (1.13) is to be invariant under local phase transformations as follows,

$$
q(x) \rightarrow U q(x) \equiv e^{i \alpha_{a}(x) T_{a}} q(x)
$$

where $U$ is an arbitrary $3 \times 3$ unitary matrix, it has the summation over the repeated suffix $a$. $T_{a}(a=1, \cdots, 8)$ is a set of linearly independent traceless $3 \times 3$ matrices, and $\alpha_{a}$ are the group parameters. The group is non-Abelian since the generators $T_{a}$ do not commute with each other,

$$
\left[T_{a}, T_{b}\right]=i f_{a b c} T_{c}
$$

where $f_{a b c}$ are real constants called the structure constants of the group. To impose $S U(3)$ local gauge invariance on the Lagrangian (1.13), the infinitesimal phase transformation is introduced,

$$
\begin{aligned}
q(x) & \rightarrow\left[1+i \alpha_{a}(x) T_{a}\right] q(x), \\
\partial_{\mu} q & \rightarrow\left(1+i \alpha_{a} T_{a}\right) \partial_{\mu} q+i T_{a} q \partial_{\mu} \alpha_{a} .
\end{aligned}
$$

The last term spoils the invariance of Lagrangian. The 8 gauge fields $G_{\mu}^{a}$ are constructed by requiring the invariance of the Lagrangian under the local gauge transformation,

$$
G_{\mu}^{a} \rightarrow G_{\mu}^{a}-\frac{1}{g} \partial_{\mu} \alpha_{a}-f_{a b c} \alpha_{b} G_{\mu}^{c}
$$


and form a covariant derivative,

$$
D_{\mu}=\partial_{\mu}+i g T_{a} G_{\mu}^{a}
$$

The gauge invariant QCD Lagrangian is formed by the replacement $\partial_{\mu} \rightarrow D_{\mu}$ in the Lagrangian (1.13), and adding a gauge invariant kinetic energy term for each of the $G_{\mu}^{a}$ fields,

$$
\begin{aligned}
\mathscr{L} & =\bar{q}\left(i \gamma^{\mu} \partial_{\mu}-m\right) q-g\left(\bar{q} \gamma^{\mu} T_{a} q\right) G_{\mu}^{a}-\frac{1}{4} G_{\mu \nu}^{a} G_{a}^{\mu \nu}, \\
G_{\mu \nu}^{a} & =\partial_{\mu} G_{\nu}^{a}-\partial_{\nu} G_{\mu}^{a}-g f_{a b c} G_{\mu}^{b} G_{\nu}^{c},
\end{aligned}
$$

(1.20) is the Lagrangian for interacting colored quarks $q$ and vector gluons $G_{\mu}$, with coupling specified by $g$. The local gauge invariance requires the gluons to be massless. The field strength $G_{\mu \nu}^{a}$ has a remarkable new property as shown in the last term in (1.21). Imposing the gauge symmetry has required that the kinetic energy term in Lagrangian is not purely kinetic but includes an induced self-interaction between the gauge bosons and reflects the fact that gluons themselves carry color charge.

\subsubsection{Electroweak Theory: $S U(2)_{L} \otimes U(1)_{Y}$}

The electroweak theory is a gauge theory unified the electromagnetic $U(1)$ and weak interactions $S U(2)$. The weak interaction typically occurs in $\beta$ decay in nuclei $(n \rightarrow$ $\left.p+\ell+\nu_{\ell}\right)$ via a $W$ boson which is weak gauge boson. The weak interaction acts only left-handed fermions, so-called $V-A$ structure, and based on $S U(2)$ isospin group with three vector bosons. The electroweak theory is suggested by Glashow [3], Weinberg [4], and Salam [5].

By demanding weak interaction, the quark fields are expressed as follows,

$$
\psi_{L}=\left(\begin{array}{l}
q_{u} \\
q_{d}
\end{array}\right)_{L}, \psi_{R}=q_{R} .
$$

The left-handed quark fields can be expressed in doublets, while the right-handed quark fields in singlets, where $q_{u}$ is up-type quarks $(u, c, t), q_{d}$ is down-type quarks $(d, s, b)$, and $q_{R}$ is six quark flavours $(u, d, c, s, t, b)$. The lepton fields are also expressed by,

$$
\psi_{L}=\left(\begin{array}{c}
\nu_{\ell} \\
\ell^{-}
\end{array}\right)_{L}, \psi_{R}=\ell_{R},
$$

where $\ell$ means three lepton flavours i.e. $e, \mu$, and $\tau$. Note that there are no righthanded neutrino fields due to satisfying $V-A$ structure in the weak interaction. Here, the free Lagrangian for the lepton and the quark fields is written in,

$$
\mathscr{L}=\sum_{j=L, R} i \bar{\psi}_{j} \gamma^{\mu} \partial_{\mu} \psi_{j}
$$


The Lagrangian (1.24) is invariant under global transformation,

$$
\begin{aligned}
& \psi_{L} \rightarrow e^{i \alpha_{a} T^{a}+i \beta Y} \psi_{L}, \\
& \psi_{R} \rightarrow e^{i \beta Y} \psi_{R},
\end{aligned}
$$

where the parameter $Y$ is hypercharge for $U(1)_{Y}$ phase transformation, the $T^{a}$ is defined by using Pauli matrices as follows,

$$
T^{a}=\frac{\tau^{a}}{2}, \tau^{1}=\left(\begin{array}{cc}
0 & 1 \\
1 & 0
\end{array}\right), \tau^{2}=\left(\begin{array}{cc}
0 & -i \\
i & 0
\end{array}\right), \tau^{3}=\left(\begin{array}{cc}
1 & 0 \\
0 & -1
\end{array}\right)
$$

and it is under $S U(2)_{L}$ transformation. The Lagrangian should be invariant under local $S U(2)_{L} \otimes U(1)_{Y}$ gauge transformation,

$$
\begin{aligned}
& \psi_{L} \rightarrow e^{i \alpha_{a}(x) T^{a}+i \beta(x) Y} \psi_{L}, \\
& \psi_{R} \rightarrow e^{i \beta(x) Y} \psi_{R} .
\end{aligned}
$$

To achieve the local gauge invariance in the Lagrangian, the derivative is replaced by covariant derivatives,

$$
\begin{aligned}
D_{\mu L} & \equiv \partial_{\mu}+i g T_{a} W_{\mu}^{a},+i \frac{g^{\prime}}{2} B_{\mu} Y \\
D_{\mu R} & \equiv \partial_{\mu}+i \frac{g^{\prime}}{2} B_{\mu} Y
\end{aligned}
$$

$D_{\mu L}\left(D_{\mu R}\right)$ is for the left(right)-handed fermion fields, $g$ is the coupling constant of $S U(2)_{L}$ and $g^{\prime}$ is of $U(1)_{Y}$. The covariant derivatives have gauge fields, $W_{\mu}^{a}(a=1,2,3)$ for $S U(2)_{L}$, and $B_{\mu}$ for $U(1)_{Y}$. The gauge fields also transform as,

$$
\begin{aligned}
B_{\mu} & \rightarrow B_{\mu}-\frac{1}{g^{\prime}} \partial_{\mu} \beta \\
\boldsymbol{W}_{\mu} & \rightarrow \boldsymbol{W}_{\mu}-\frac{1}{g} \partial_{\mu} \boldsymbol{\alpha}-\boldsymbol{\alpha} \times \boldsymbol{W}_{\mu} .
\end{aligned}
$$

In addition, the gauge field strength tensors are introduced by requiring the local gauge invariant,

$$
\begin{aligned}
B_{\mu \nu} & \equiv \partial_{\mu} B_{\nu}-\partial_{\nu} B_{\mu} \\
W_{\mu \nu}^{a} & \equiv \partial_{\mu} W_{\nu}^{a}-\partial_{\nu} W_{\mu}^{a}-g \varepsilon_{a b c} W_{\mu}^{b} W_{\nu}^{c} .
\end{aligned}
$$

Finally, the Lagrangian under local gauge invariant in electroweak interaction can be written as,

$$
\mathscr{L}=\sum_{j=L, R} i \bar{\psi}_{j} \gamma^{\mu} D_{\mu, j} \psi_{j}-\frac{1}{4} W_{\mu \nu}^{a} W_{a}^{\mu \nu}-\frac{1}{4} B_{\mu \nu} B_{\mu \nu}
$$


Although the weak and electromagnetic interactions coexist in the $S U(2)_{L} \otimes U(1)_{Y}$ gauge symmetry, it describes no realistic world, because there are no mass terms for fermions and weak gauge bosons which are known that they are massive, and weak interaction only affects in short range. However introducing the mass terms such as $\frac{1}{2} M_{W}^{2} W_{\mu} W^{\mu}$ in the Lagrangian breaks the gauge symmetry. The fermion terms also break due to different transformation between the left-handed and right-handed fermion fields,

$$
m_{f} \bar{f} f=m_{f}\left(\bar{f}_{R} f_{L}+\bar{f}_{L} f_{R}\right)
$$

using the left-handed and right handed relation equations,

$$
f_{L}=\frac{1}{2}\left(1-\gamma^{5}\right) f, f_{R}=\frac{1}{2}\left(1+\gamma^{5}\right) f .
$$

Fortunately, the nature have a solution(mechanism) to be invariant under gauge transformation when the Lagrangian has a mass terms for fermion and weak gauge boson, so-called "Spontaneous symmetry breaking".

\subsubsection{Spontaneous Symmetry Breaking}

To give mass to the gauge bosons and fermions, the electroweak gauge symmetry are hidden. Here let us start by introducing the scalar real field $\phi$ as simple example, and its Lagrangian is written by,

$$
\begin{aligned}
\mathscr{L} & =\frac{1}{2} \partial_{\mu} \phi \partial^{\mu} \phi-V(\phi), \\
V(\phi) & =\frac{1}{2} \mu^{2} \phi^{2}+\frac{1}{4} \lambda \phi^{4},
\end{aligned}
$$

where $\lambda>0$, the Lagrangian is invariant under the symmetry operation: $\phi \rightarrow-\phi$.

If $\mu^{2}>0$, it can be regarded that the Lagrangian describes a scalar fields with mass $\mu$, the $\phi^{4}$ terms means self-interaction with coupling $\lambda$, and the minimum of the potential $V(\phi)$ is,

$$
\langle 0|\phi| 0\rangle \equiv \phi_{0}=0
$$

as shown in the left side of Figure 1.1. On the other hand, if $\mu^{2}<0$, the potential $V(\phi)$ has a minimum when,

$$
\begin{gathered}
\frac{\partial V}{\partial \phi}=\mu^{2} \phi+\lambda \phi^{3}=0, \\
\left\langle 0\left|\phi^{2}\right| 0\right\rangle \equiv \phi_{0}^{2}=-\frac{\mu^{2}}{\lambda} \equiv v^{2},
\end{gathered}
$$


as shown in the right side of Figure 1.1. The value $v=\sqrt{\mu^{2} / \lambda}$ is called "vacuum expectation value" of the scalar field $\phi$. Here the field $\phi$ is expanded around the minimum value $v$ with the quantum fluctuation $\eta$,

$$
\phi=v+\eta
$$

From this, the Lagrangian (1.40) becomes

$$
\mathscr{L}=\frac{1}{2} \partial_{\mu} \eta \partial^{\mu} \eta-\lambda v^{2} \eta^{2}-\lambda v \eta^{3}-\frac{1}{4} \eta^{4}+\text { const }
$$

where a scalar field $\eta$ with mass $m_{\eta}=\sqrt{-2 \mu^{2}}$ appears in the Lagrangian (1.45), and there are self-interaction terms $\eta^{3}$ and $\eta^{4}$, in particular, the cubic term breaks the symmetry in the Lagrangian without external operation, it is called "Spontaneous Symmetry Breaking (SSB)". Next step, let us introduce the SSB to electroweak symmetry to get the true world picture, that is, the weak bosons and the fermions are massive.
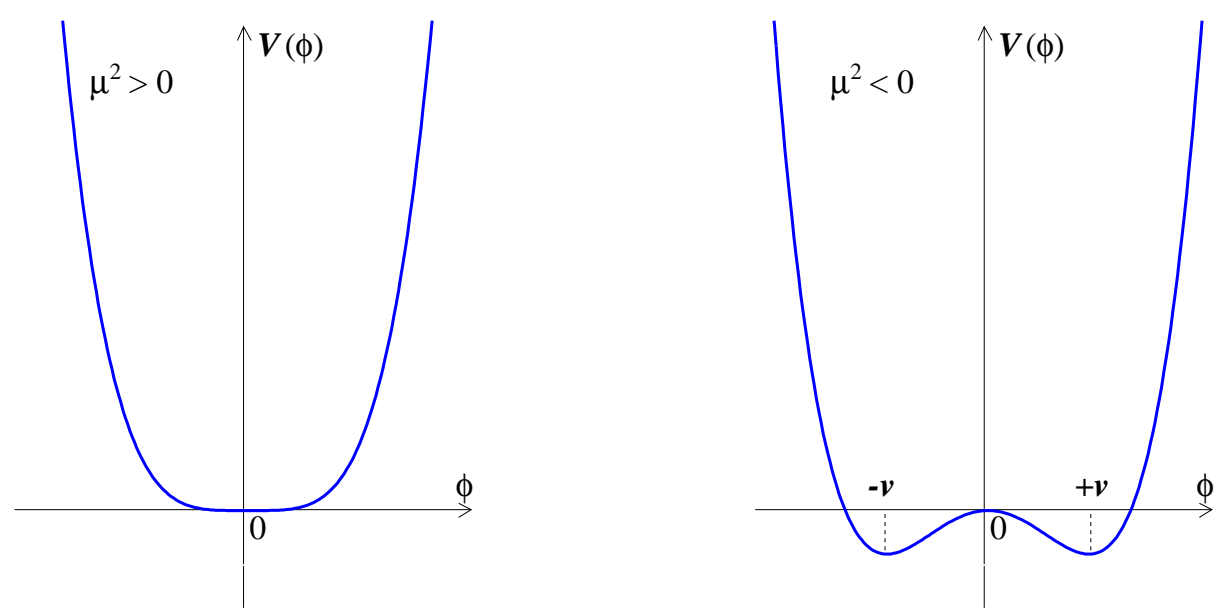

Figure 1.1: The potential $V(\phi)$ of the scalar field $\phi$ in the case $\mu^{2}>0$ (left) and $\mu^{2}<0$ (right).

\subsubsection{Higgs Mechanism}

As described in $\$ 1.1 .4$, however the Lagrangian (1.36) is invariant under local gauge invariant, the Lagrangian describes the no real world picture because the weak gauge bosons and fermions have no mass in the Lagrangian. But the Lagrangian is broken by including the mass terms. Now, let us show that the Lagrangian becomes the real 
world Lagrangian by using the symmetry breaking $[6,7,8]$. By introducing complex scalar doublet,

$$
\phi=\left(\begin{array}{c}
\phi^{+} \\
\phi^{0}
\end{array}\right)=\frac{1}{\sqrt{2}}\left(\begin{array}{c}
\phi_{1}+i \phi_{2} \\
\phi_{3}+i \phi_{4}
\end{array}\right), Y_{\phi}=+1,
$$

where the hypercharge is 1 for the scalar fields, the Lagrangian can be written by

$$
\mathscr{L}=\left(\partial_{\mu} \phi\right)^{\dagger}\left(\partial^{\mu} \phi\right)-\mu^{2} \phi^{\dagger} \phi-\lambda\left(\phi^{\dagger} \phi\right)^{2}
$$

In this case, if $\mu^{2}<0$, the vacuum expectation value and the scalar field after the symmetry breaking with the real scalar field $h$ become as follows,

$$
\begin{aligned}
\phi^{\dagger} \phi & =\frac{\phi_{1}^{2}+\phi_{2}^{2}+\phi_{3}^{2}+\phi_{4}^{2}}{2}=\frac{-\mu^{2}}{2 \lambda} \equiv \frac{v^{2}}{2}, \\
\phi & =\frac{1}{\sqrt{2}}\left(\begin{array}{c}
0 \\
v+h
\end{array}\right),
\end{aligned}
$$

where the scalar fields are chosen as $\phi^{1}=\phi^{2}=\phi^{4}=0$, and $\phi^{3}=v$. Let us expand the first term of the Lagrangian (1.47), i.e. the kinematical terms,

$$
\begin{aligned}
\left|D_{\mu} \phi\right|^{2} & =\left|\left(\partial_{\mu}-i g T^{a} W_{\mu}^{a}+i \frac{g^{\prime}}{2} B_{\mu}\right) \phi\right|^{2} \\
& =\frac{1}{2}\left(\partial_{\mu} h\right)^{2}+\frac{g^{2} v^{2}}{4}\left|\frac{W_{\mu}^{1}+i W_{\mu}^{2}}{2}\right|^{2}+\frac{v^{2}}{8}\left|g W_{\mu}^{3}-g^{\prime} B_{\mu}\right|^{2}+\text { interaction terms }
\end{aligned}
$$

where the derivative is replaced to covariant derivative (1.31), and define the field $W_{\mu}^{ \pm}$, $Z_{\mu}$ and $A_{\mu}$ written as follows,

$$
\begin{aligned}
W_{\mu}^{ \pm} & =\frac{1}{\sqrt{2}}\left(W_{\mu}^{1} \pm i W_{\mu}^{2}\right), \\
Z_{\mu} & =W_{\mu}^{3} \cos \theta_{W}-B_{\mu} \sin \theta_{W}, \\
A_{\mu} & =W_{\mu}^{3} \sin \theta_{W}+B_{\mu} \cos \theta_{W},
\end{aligned}
$$

where weak mixing angle $\theta_{W}$ is defined as $g^{\prime}=g \tan \theta_{W}$, the $A_{\mu}$ field is the orthogonal field to the $Z_{\mu}$ field, and the masses of fields can be expressed as respectively,

$$
M_{W}=\frac{1}{2} v g, M_{Z}=\frac{1}{2} v \sqrt{g^{2}+g^{\prime 2}}, M_{A}=0 .
$$

Note that the $W_{\mu}$ and the $Z_{\mu}$ fields become massive, while the $A_{\mu}$ field is still massless, that is, the weak gauge bosons can have desirable mass by introducing the SSB, in particular, it is called "Higgs Mechanism". By using the weak mixing angle $\theta_{W}$, the $W_{\mu}$ field is related to $Z_{\mu}$ field as follows,

$$
M_{W}=M_{Z} \cos \theta_{W}
$$


The fermion fields should be massive to achieve the true world in the electroweak Lagrangian. The Higgs mechanism also gives a mass to the fermions under the local gauge invariant. The Lagrangian with fermion fields is written by,

$$
\mathscr{L}_{\text {Yukawa }}=-G_{f} \bar{\psi}_{L} \phi \psi_{R}-G_{f} \bar{\psi}_{R} \phi^{\dagger} \psi_{L}
$$

where $G_{f}$ is arbitrary constant for each fermion. First, the lepton sector Lagrangian becomes,

$$
\begin{aligned}
\mathscr{L}_{\text {lepton }} & =-G_{\ell}\left[\left(\bar{\nu}_{\ell}, \bar{\ell}\right)_{L}\left(\begin{array}{c}
\psi^{+} \\
\psi^{0}
\end{array}\right) \ell_{R}+\bar{\ell}_{R}\left(\psi^{-}, \bar{\psi}^{0}\right)\left(\begin{array}{c}
\nu_{\ell} \\
\ell
\end{array}\right)_{L}\right] \\
& =-\frac{G_{\ell}}{\sqrt{2}} v\left(\bar{\ell}_{L} \ell_{R}+\bar{\ell}_{R} \ell_{L}\right)-\frac{G_{\ell}}{\sqrt{2}}\left(\bar{\ell}_{L} \ell_{R}+\bar{\ell}_{R} \ell_{L}\right) h \\
& =-m_{\ell} \bar{\ell} \ell-\frac{m_{\ell}}{v} \bar{\ell} \ell h,
\end{aligned}
$$

using (1.38) and $m_{\ell}=G_{\ell} v / \sqrt{2}$ is defined as the lepton mass. The lepton sector Lagrangian (1.57) then keeps the gauge symmetry under the local transformation. Let us show that the quark sector Lagrangian also becomes the invariant. In the quark sector, the new higgs doublet must be introduced by using $\phi$ to give the the up-type quark mass,

$$
\phi_{c}=i \tau_{2} \phi=\left(\begin{array}{c}
-\bar{\phi}^{0} \\
\phi^{-}
\end{array}\right)
$$

the higgs doublet is chosen the following after the symmetry breaking,

$$
\phi_{c}=\frac{1}{\sqrt{2}}\left(\begin{array}{c}
v+h \\
0
\end{array}\right)
$$

The quark sector Lagrangian is formed by

$$
\begin{aligned}
\mathscr{L}_{\text {quark }} & =-G_{d}(\bar{u}, \bar{d})_{L}\left(\begin{array}{c}
\psi^{+} \\
\psi^{0}
\end{array}\right) d_{R}-G_{u}(\bar{u}, \bar{d})_{L}\left(\begin{array}{c}
-\bar{\psi}^{0} \\
\psi^{-}
\end{array}\right) u_{R}+h . c . \\
& =-m_{d} \bar{d} d-m_{u} \bar{u} u-\frac{m_{d}}{v} \bar{d} d h-\frac{m_{u}}{v} \bar{u} u h,
\end{aligned}
$$

where the down-type and the up-type quark masses are defined as $m_{d}=G_{d} v / \sqrt{2}$ and $m_{u}=G_{u} v / \sqrt{2}$ respectively. The quark sector Lagrangian also preserves the gauge invariant after the symmetry breaking.

The Higgs Mechanism gives us the electroweak Lagrangian with relevant field mass terms under the gauge symmetry. However the fermions masses are not predicted in the Lagrangian because of general fermion coupling $G_{f}$ 's are arbitrary constant, and the Higgs mass and its potential structure are also unknown. They need to be revealed by a experiment. 


\subsubsection{Two Higgs Doublet Model}

In the previous section, the minimal SM higgs is considered, however the scalar field doublet can be introduced as two, and more doublets to break the electroweak symmetry. Then, let us introduce the two complex doublet scalar fields $\phi_{1}$ and $\phi_{2}$ so-called "Two Higgs Doublet Model (2HDM)",

$$
\phi_{1}=\left(\begin{array}{c}
\phi_{1}^{+} \\
\phi_{1}^{0}
\end{array}\right), \phi_{2}=\left(\begin{array}{c}
\phi_{2}^{+} \\
\phi_{2}^{0}
\end{array}\right)
$$

The 2HDM have mainly two scenarios, named "Type-I" and "Type-II", respectively. In the "Type-I", the scalar fields $\phi_{1}$ do not couple to any quarks and leptons, while the other scalar fields $\phi_{2}$ couple to them. In the "Type-II", the first scalar fields $\phi_{1}$ couple only to down-type quarks and leptons, while the second scalar fields $\phi_{2}$ couple only to up-type quarks. In this thesis, the Type-I scenario is noticed $[10,11]$.

First, let us start to introduce the most general potential written as,

$$
\begin{aligned}
V\left(\phi_{1}, \phi_{2}\right)= & \lambda_{1}\left(\phi_{1}^{\dagger} \phi_{1}-v_{1}^{2}\right)^{2}+\lambda_{2}\left(\phi_{2}^{\dagger} \phi_{2}-v_{2}^{2}\right)^{2} \\
& +\lambda_{3}\left[\left(\phi_{1}^{\dagger} \phi_{1}-v_{1}^{2}\right)+\left(\phi_{2}^{\dagger} \phi_{2}-v_{2}^{2}\right)\right]^{2} \\
& +\lambda_{4}\left[\left(\phi_{1}^{\dagger} \phi_{1}\right)\left(\phi_{2}^{\dagger} \phi_{2}\right)-\left(\phi_{1}^{\dagger} \phi_{2}\right)\left(\phi_{2}^{\dagger} \phi_{1}\right)\right] \\
& +\lambda_{5}\left[\operatorname{Re}\left(\phi_{1}^{\dagger} \phi_{2}\right)-v_{1} v_{2} \cos \xi\right]^{2}+\lambda_{6}\left[\operatorname{Im}\left(\phi_{1}^{\dagger} \phi_{2}\right)-v_{1} v_{2} \sin \xi\right]^{2} \\
& +\lambda_{7}\left[\operatorname{Re}\left(\phi_{1}^{\dagger} \phi_{2}\right)-v_{1} v_{2} \cos \xi\right]\left[\operatorname{Im}\left(\phi_{1}^{\dagger} \phi_{2}\right)-v_{1} v_{2} \sin \xi\right]
\end{aligned}
$$

where $\lambda_{i}$ are real parameters, and the potential has a discrete symmetry, $\phi_{1} \rightarrow-\phi_{1}$, only broken softly. The last term with $\lambda_{7}$ can be eliminated by defining the phases of the scalar fields or demanding the CP-conservation which is assumed in this section.

The vacuum expectation values for the two scalar fields after symmetry breaking are formed as follows,

$$
\left\langle\phi_{1}\right\rangle=\left(\begin{array}{c}
0 \\
v_{1}
\end{array}\right),\left\langle\phi_{2}\right\rangle=\left(\begin{array}{c}
0 \\
v_{2}
\end{array}\right)
$$

where $v_{1,2}$ are real, in addition, the vacuum expectation values are defined by,

$$
\tan \beta \equiv \frac{v_{2}}{v_{1}}, v^{2} \equiv v_{1}^{2}+v_{2}^{2}=\frac{2 m_{W}^{2}}{g^{2}}=(173 \mathrm{GeV})^{2},
$$

as discussed below, the parameter $\beta$ serves as key role in the model. In this model, there are five Higgs boson $\left(h^{0}, H^{0}, A^{0}\right.$, and $\left.H^{ \pm}\right)$and three Goldstone bosons $\left(G^{0}\right.$ and $G^{ \pm}$) which give a mass to weak bosons. The charged Goldstone boson $G^{ \pm}$is orthogonal to the charged Higgs boson $H^{ \pm}$, and the charged sector are expressed by,

$$
\begin{aligned}
G^{ \pm} & =\phi_{1}^{ \pm} \cos \beta+\phi_{2}^{ \pm} \sin \beta, \\
H^{ \pm} & =-\phi_{1}^{ \pm} \sin \beta+\phi_{2}^{ \pm} \cos \beta .
\end{aligned}
$$


By demanding the CP-conservation, the imaginary parts and the real parts of the neutral scalar fields decouple. The neutral Goldstone boson is orthogonal to the one of the neutral higgs bosons, the imaginary sector (CP-odd) are obtained as,

$$
\begin{aligned}
G^{0} & =\sqrt{2}\left[\operatorname{Im}\left(\phi_{1}^{0}\right) \cos \beta+\operatorname{Im}\left(\phi_{2}^{0}\right) \sin \beta\right], \\
A^{0} & =\sqrt{2}\left[-\operatorname{Im}\left(\phi_{1}^{0}\right) \sin \beta+\operatorname{Im}\left(\phi_{2}^{0}\right) \cos \beta\right],
\end{aligned}
$$

while the real sector (CP-even) are expressed by as follows,

$$
\begin{aligned}
H^{0} & =\sqrt{2}\left[\left(\operatorname{Re}\left(\phi_{1}^{0}\right)-v_{1}\right) \cos \alpha+\left(\operatorname{Re}\left(\phi_{2}^{0}\right)-v_{2}\right) \sin \alpha\right], \\
h^{0} & =\sqrt{2}\left[-\left(\operatorname{Re}\left(\phi_{1}^{0}\right)-v_{1}\right) \sin \alpha+\left(\operatorname{Re}\left(\phi_{2}^{0}\right)-v_{2}\right) \cos \alpha\right],
\end{aligned}
$$

where the neutral higgs scalars are related with the mixing angle $\alpha$ which is defined as,

$$
\begin{aligned}
\sin 2 \alpha & =\frac{2 \mathcal{M}_{12}}{\sqrt{\left(\mathcal{M}_{11}-\mathcal{M}_{22}\right)^{2}+4 \mathcal{M}_{12}^{2}}}, \\
\cos 2 \alpha & =\frac{\mathcal{M}_{11}-\mathcal{M}_{22}}{\sqrt{\left(\mathcal{M}_{11}-\mathcal{M}_{22}\right)^{2}+4 \mathcal{M}_{12}^{2}}},
\end{aligned}
$$

then, the scalar masses can be obtained by diagonalizing the Higgs boson matrix, $M_{i j}^{2}=\frac{\partial^{2} V}{\partial \phi_{i} \partial \phi_{j}}$, the Higgs masses are respectively written as,

$$
\begin{aligned}
M_{H^{0}, h^{0}}^{2} & =\frac{1}{2}\left[\mathcal{M}_{11}+\mathcal{M}_{22} \pm \sqrt{\left(\mathcal{M}_{11}-\mathcal{M}_{22}\right)^{2}+4 \mathcal{M}_{12}^{2}}\right], \\
M_{H^{ \pm}}^{2} & =\lambda_{4}\left(v_{1}^{2}+v_{2}^{2}\right), M_{A^{0}}^{2}=\lambda_{6}\left(v_{1}^{2}+v_{2}^{2}\right),
\end{aligned}
$$

where using the mass matrices defined as follows,

$$
\mathcal{M}=\left(\begin{array}{cc}
\mathcal{M}_{11} & \mathcal{M}_{12} \\
\mathcal{M}_{12} & \mathcal{M}_{22}
\end{array}\right)=\left(\begin{array}{cc}
4 v_{1}^{2}\left(\lambda_{1}+\lambda_{3}\right)+v_{2}^{2} \lambda_{5} & \left(4 \lambda_{3}+\lambda_{5}\right) v_{1} v_{2} \\
\left(4 \lambda_{3}+\lambda_{5}\right) v_{1} v_{2} & 4 v_{2}^{2}\left(\lambda_{2}+\lambda_{3}\right)+v_{1}^{2} \lambda_{5}
\end{array}\right) .
$$

The neutral Higgs boson couplings relate to the vacuum expectation value ratio $\beta$ and the mixing angle $\alpha$, the coupling can be repressed by,

$$
\frac{g_{h^{0} V V}}{g_{\phi^{0} V V}}=\sin (\beta-\alpha), \frac{g_{H^{0} V V}}{g_{\phi^{0} V V}}=\cos (\beta-\alpha),
$$

where $\phi^{0}$ is minimal SM Higgs boson, and $V=W^{ \pm}, Z$. Note that the remaining neutral Higgs boson $A^{0}$ couples to no gauge boson.

Next, let us show the Higgs-fermion interaction in the Type-I. The interaction is formed as,

$$
\begin{aligned}
\mathscr{L}_{\text {fermion }}= & -\frac{g}{2 M_{W} \sin \beta} \bar{D} M_{D} D\left(H^{0} \sin \alpha+h^{0} \cos \alpha\right)-\frac{i g \cot \beta}{2 M_{W}} \bar{D} M_{D} \gamma^{5} D A^{0} \\
& -\frac{g}{2 M_{W} \sin \beta} \bar{U} M_{U} U\left(H^{0} \sin \alpha+h^{0} \cos \alpha\right)+\frac{i g \cot \beta}{2 M_{W}} \bar{U} M_{U} \gamma^{5} U A^{0} \\
& +\frac{g \cot \beta}{2 \sqrt{2} M_{W}}\left(\bar{U}\left[M_{U} K\left(1-\gamma^{5}\right)-K M_{D}\left(1+\gamma^{5}\right)\right] D H^{+}+\text {h.c. }\right)+\text { leptonic sector. }
\end{aligned}
$$


where $M_{U}$ and $M_{D}$ are diagonal quark matrices, $K$ is Kobayashi-Maskawa mixing matrix. The Higgs-lepton couplings can be expressed by replacing $U, D$ and the quark mass matrices with the corresponding lepton fields and lepton matrices and setting $K=1$. The neutral Higgs boson couplings to gauge bosons and fermions are shown in Table 1.3. In particular, the "fermiophobic Higgs $\left(h_{F}\right)$ " appears in the 2HDM Type-I by setting the mixing angle,

$$
\alpha=\frac{\pi}{2}
$$

as seen in (1.77), so-called "fermiophobia". The "fermiophobic Higgs" becomes only coupling to the bosons.

\begin{tabular}{cccc}
\hline \hline$\phi$ & $H^{0}$ & $h^{0}$ & $A^{0}$ \\
\hline$g_{\phi V V}$ & $\cos (\beta-\alpha)$ & $\sin (\beta-\alpha)$ & 0 \\
$g_{\phi \bar{u} u}$ & $\frac{\sin \alpha}{\sin \beta}$ & $\frac{\cos \alpha}{\sin \beta}$ & $\cot \beta$ \\
$g_{\phi \bar{d} d}$ & $\frac{\sin \alpha}{\sin \beta}$ & $\frac{\cos \alpha}{\sin \beta}$ & $\cot \beta$ \\
$g_{\phi \bar{e} e}$ & $\frac{\sin \alpha}{\sin \beta}$ & $\frac{\cos \alpha}{\sin \beta}$ & $\cot \beta$ \\
\hline \hline
\end{tabular}

Table 1.3: The neutral Higgs boson couplings to gauge bosons $\left(V=W^{ \pm}, Z\right)$ and fermions in 2HDM Type-I.

\subsection{Production of The Higgs Boson at Tevatron}

As mentioned before, the SM Higgs boson is that particle give a mass to any particles except for weak gauge boson photon and neutrinos, that is, the higgs boson couples to the massive particles with its production and decay. The strength of the process depend on higgs mass and relevant particle masses.

The main production mechanisms for the SM Higgs at hadron collider uses coupling to the heavy particles, i.e. $W, Z$ bosons, and top quark. There are 4 dominant SM Higgs boson production modes in the Tevatron, the gluon-gluon fusion production, the associated production with the weak boson, the vector boson fusion (VBF) production, and the associated production with top quark, as shown in Figure 1.2. However there are also several mechanisms for the pair Higgs boson production, the mechanism is not useful production in the Tevatron because the production cross-section will extremely become small by the additional coupling.

In this section, the production mechanisms are described, and also its cross-sections in the Tevatron are mentioned which are taken QCD correction on NLO into account $[12,13]$. 

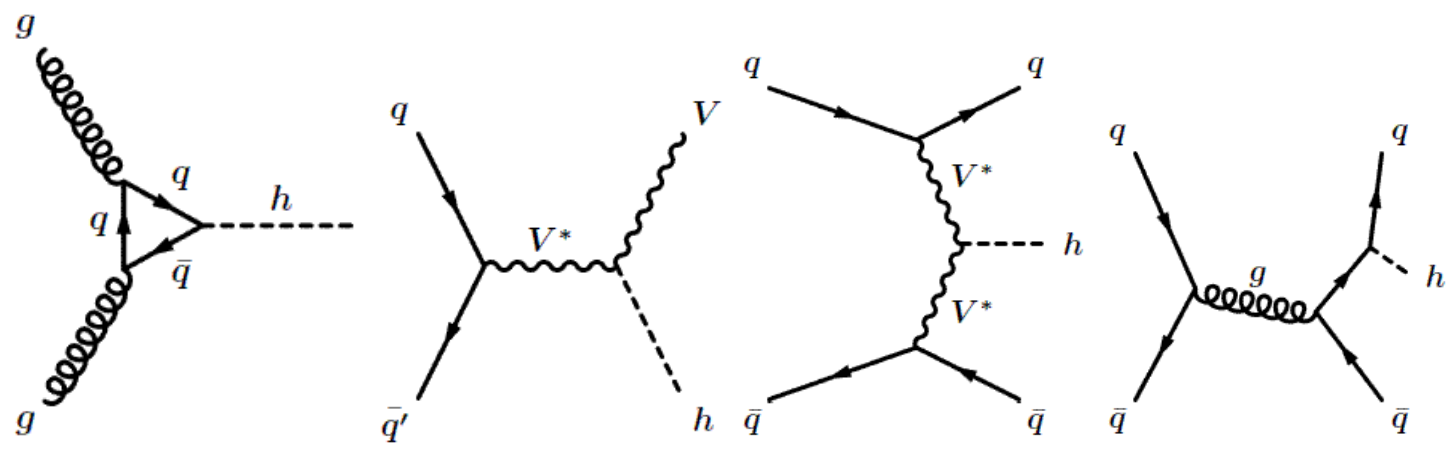

Figure 1.2: Feynman diagrams for the Higgs boson production mechanism (from left to right: the gluon-gluon fusion, the associated production with vector boson, the vector boson fusion, and the associated production with heavy quarks).

\subsubsection{The gluon-gluon Fusion Production}

The Higgs boson production in the gluon-gluon fusion mechanism is mediated by triangular loop of the heavy quarks, in the SM, the top quarks mostly contribute the process, while the bottom quark loop is also non-negligible contribution. In the single Higgs production, the two loop QCD radiative corrections enhance the cross-section 60-100\%. The production process is the largest cross-section in the Tevatron, as shown in Figure 1.3, 1.0-0.2 pb from 100 to $200 \mathrm{GeV} / c^{2}$.

\subsubsection{The Associated Production with Vector Boson}

The associated production process goes on with $q \bar{q}$ annihilation into a virtual vector boson $V^{*}$, and then, do the "Higgs-strahlung". The QCD corrections increases the cross-section $30 \%$. The cross-section for a virtual $W^{*}$ process, $q \bar{q} \rightarrow W h$, is roughly a factor of two higher than for a virtual $Z$ ones, as shown in Figure 1.3. The cross-section for Wh mechanism is $0.3-0.02 \mathrm{pb}$ from 100 to $200 \mathrm{GeV} / c^{2}$, and for $Z h, 0.2-0.01 \mathrm{pb}$. The production mechanism is most promising discovery ones for $M_{h}<130 \mathrm{GeV} / c^{2}$.

\subsubsection{The Vector Boson Fusion Production}

The vector boson fusion mechanism is that the quark and anti-quark both radiate virtual bosons, and then the bosons annihilate to produce the Higgs boson. The QCD corrections enhance the cross-section by about $10 \%$. The cross-section is $0.1-0.02 \mathrm{pb}$ from 100 to $200 \mathrm{GeV} / c^{2}$. In this production process, there are two forward jets, it can somewhat suppress the QCD backgrounds by using the distinct kinematics.

\subsubsection{The Associated Production with Heavy Quarks}

The production process where Higgs is produced association with heavy quark pairs with the final state quarks being either the top or the bottom quark. At tree-level, it 
originates from $q \bar{q}$ annihilation into heavy quarks with the Higgs boson emitted from the quarks lines which is the mains source at the Tevatron. Figure 1.3 shows of the top quark pairs, the QCD corrections are taken the limit of $M_{h} \ll M_{t}$ into account.

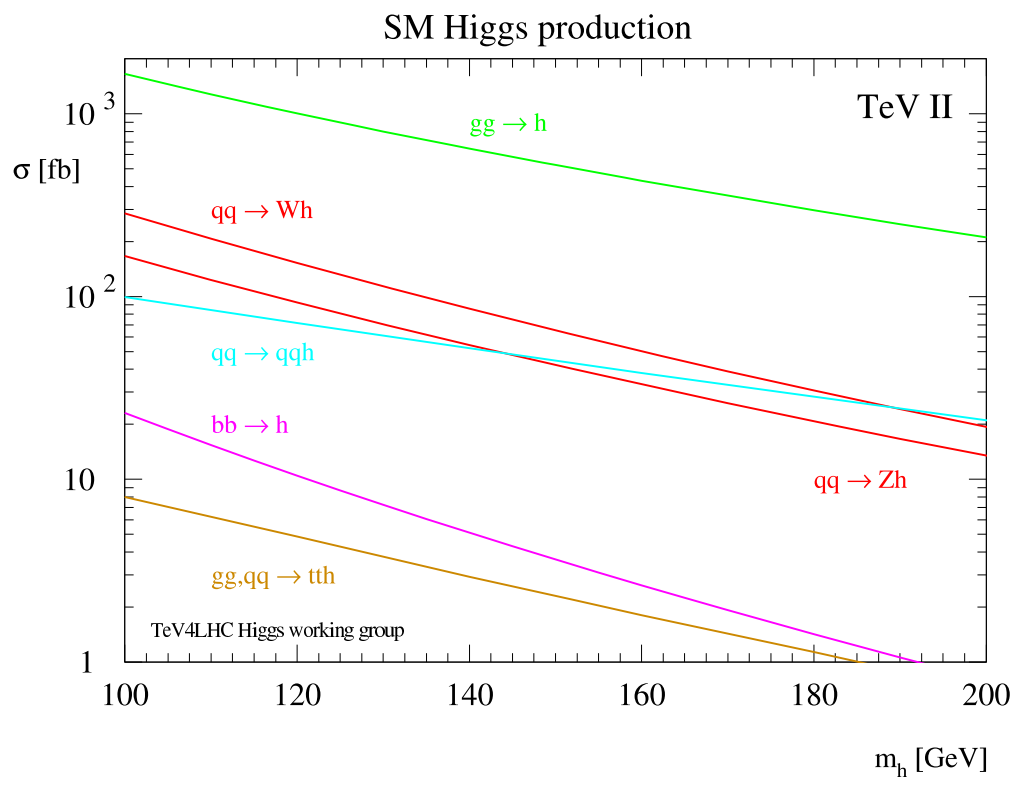

Figure 1.3: Production cross-section of the SM Higgs boson in several processes at the Tevatron.

\subsection{Decay of The Higgs Boson at Tevatron}

In the decay process, the Higgs boson has the tendency to decay into the heaviest particles allowed by phase space, because the Higgs couplings to gauge bosons and fermions are proportional to the masses of the particles.

The branching fractions for the dominant decay modes of the Higgs boson are varied by the Higgs-self mass. In the Tevatron, the Higgs hunters chose the best set of the Higgs decay mode and production mechanism as mentioned previous section to maximally get the discovery chance.

In this below section, the several decay modes are discussed, which are used in the Higgs search at the Tevatron. Figure 1.4 shows the branching fraction of the dominant decay modes of the minimal Standard Model Higgs boson and the fermiophobic Higgs in 2HDM Type-I. 


\subsection{1 $h \rightarrow f \bar{f}$ Mode}

The decay mode $h \rightarrow b \bar{b}$ dominates for the minimal SM Higgs boson below about 130 $\mathrm{GeV} / c^{2}$. The $b \bar{b}$ decay mode is ubiquitous employed in the Higgs search at the Tevatron, in particular, in the associated production with vector boson channel, because there are distinctive signatures in the final state, i.e. leptons plus two-bjets, which lead to not too large backgrounds, and the valid feature can reconstructs the $b \bar{b}$ invariant mass peak using flavour tagging (b-tagging) to reject the background such as $W b \bar{b}$ and $t \bar{t}$ production event. The decay mode $h \rightarrow \tau \tau$ also is somewhat valid with respect to its high branching fraction for low mass minimal SM Higgs boson if enough luminosity is available, however needs a significant improvement of $\tau$ identification.

\subsection{2 $h \rightarrow W W^{(*)}$ Mode}

The decay mode has one of the most promising detection and the potential discovery final state $\ell^{+} \nu \ell^{-} \nu$ for the minimal SM Higgs boson $M_{h}>135 \mathrm{GeV} / c^{2}$ combination using gluon-gluon fusion production in the Tevatron. The dominant background source is $W W$ and $t \bar{t}$ production. The decay process in the latter involves $t \rightarrow b W$, i.e. become 2-lepton plus 2-bjets final state, although 2-bjets non-requirement can greatly removes the background. The former can be removed by using the characteristic spincorrelations in the Higgs channel. With associated production with vector boson, the decay mode will be significance detection channel using like-sign dilepton event in the final state, which excellently remove the QCD and electroweak backgrounds. The combination channel are employed in this thesis, and also useful to search for the fermiophobic Higgs from its low mass region due to its feature, as shown in the right-hand side of Figure 1.4.

\subsection{3 $\quad h \rightarrow \gamma \gamma$ Mode}

Although the two photon decay mode is extremely rare for the minimal SM Higgs boson at the Tevatron, for low mass fermiophobic Higgs will be useful, which will be dominant mode, as shown in Figure 1.4. And the mode give a narrow mass peak which can effectively reject the backgrounds.

\subsection{Higgs Boson Mass Constraints}

As mentioned before, the Higgs boson mass are not given in the SM framework while predicted the existence of the Higgs boson. However the Higgs boson mass can be constraint by taking into account adaptive limit for perturbation theory. In additional, the passed experiment results have constrained the Higgs boson mass. In this section, the constraints on the Higgs boson mass are discussed in both theoretical and experimental point of view. 

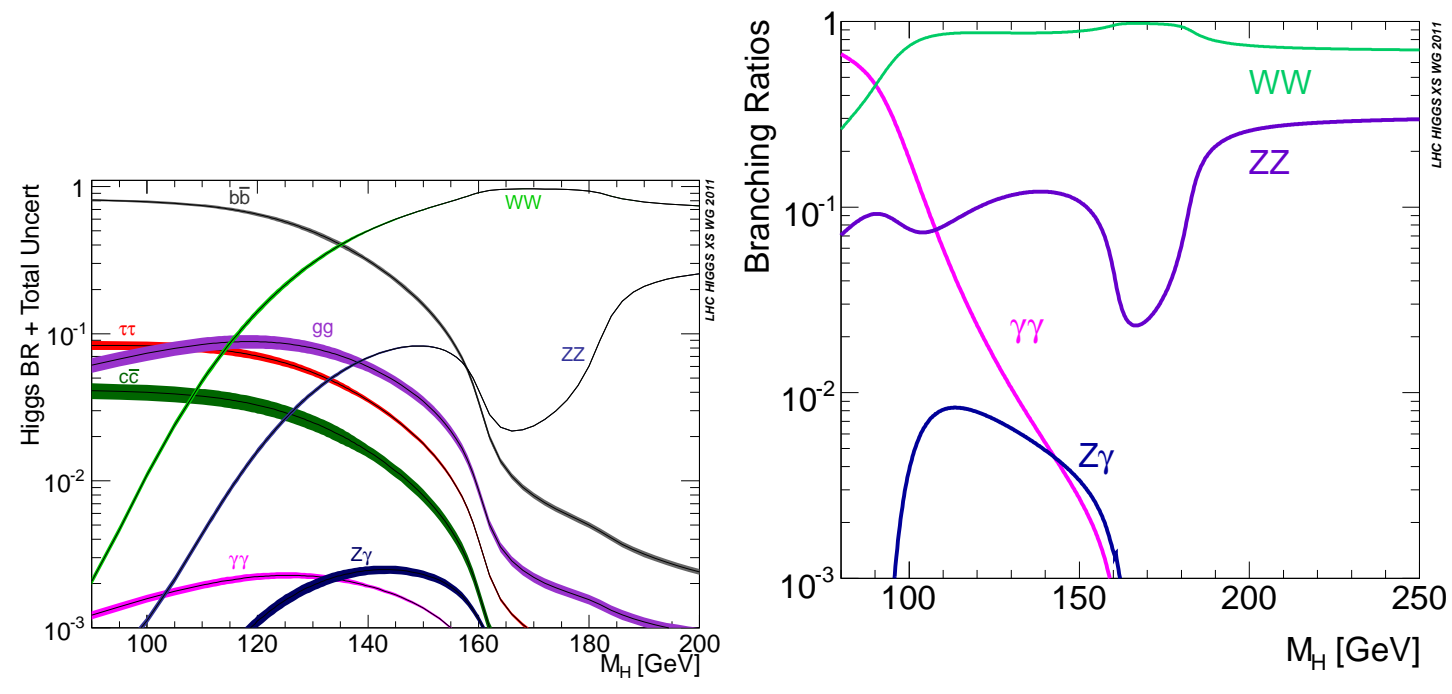

Figure 1.4: The branching fraction on the minimal SM Higgs boson (left-side) and the Fermiophobic Higgs boson in 2HDM Type-I (right-side).

\subsubsection{Theoretical Higgs Boson Mass Constraints}

The Higgs mass and its coupling depend on the considered energy because of quantum (radiative) corrections. The Higgs mass can be limited by taking into account the energy scale from upper side (triviality bound) and lower side (vacuum stability bound) where the SM is valid within perturbation theory. In this section, let us see the theoretical constraint of the Higgs boson mass. Figure 1.7 shows the upper bound and the lower bound on the Higgs boson mass as a function of the energy scale $\Lambda_{c}$.

\section{Triviality Bound}

First, let us take the one-loop radiative correction to the Higgs boson quartic coupling for the contributions to the Higgs boson. The Feynman diagrams for the tree-level and the one-loop corrections to the Higgs boson self-coupling are shown in Figure 1.5. The variation of the quartic Higgs coupling with the energy scale $Q$ is described by the Renormalization Group Equation (RGE),

$$
\frac{d \lambda\left(Q^{2}\right)}{d Q^{2}}=\frac{3}{4 \pi^{2}} \lambda^{2}\left(Q^{2}\right)+\text { higher order. }
$$

The solution of the equation by selecting a energy point to be the electroweak symmetry breaking scale $Q_{0}=v$ can be written by,

$$
\lambda\left(Q^{2}\right)=\lambda\left(v^{2}\right)\left[1-\frac{3}{4 \pi^{2}} \lambda\left(v^{2}\right) \log \frac{Q^{2}}{v^{2}}\right]^{-1} .
$$

The quartic couplings varies logarithmically with the squared energy $Q^{2}$. From (1.80), if the energy is much smaller than the electroweak breaking scale, $Q^{2} \ll v^{2}$, the quartic 
coupling becomes extremely small and eventually vanishes, i.e. the theory is trivially non-interacting, $\lambda\left(Q^{0}\right) \sim 0$. While when the energy is much higher than electroweak scale, $Q^{2} \gg v^{2}$, the quartic coupling grows and eventually becomes infinite $\lambda\left(Q^{2}\right) \gg 1$ which is called Landau Pole, i.e. the coupling becomes infinite at the energy,

$$
\Lambda_{c}=v \exp \left(\frac{4 \pi^{2} v^{2}}{M_{h}^{2}}\right)
$$

The energy point $\Lambda_{c}$ is corresponding to the Higgs mass upper limit to avoid the Landau pole as seen in (1.81). For instance, if the energy $\Lambda_{c} \sim 10^{16}$, the Higgs boson mass need to be light, $M_{h}<200 \mathrm{GeV} / c^{2}$, while when the energy $\Lambda_{c} \sim 10^{3}$, the Higgs boson mass is allowed to be the order of $1 \mathrm{TeV} / c^{2}$.
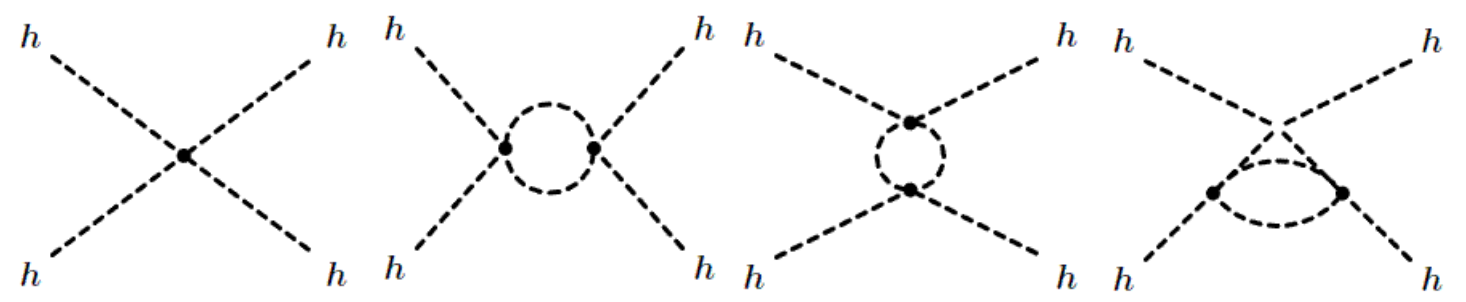

Figure 1.5: Feynman diagrams for the tree-level and one-loop Higgs self-coupling.

\section{Stability Bound}

Next, the Higgs boson coupling needs to also include the contribution from fermion and gauge bosons. In this case, only the contribution of top quark and massive gauge bosons are taken into account because the Higgs boson coupling are proportional to the particle masses. The Feynman diagrams for the top quark and gauge boson contribution are shown in Figure 1.6. The one-loop RGE for the quartic coupling including the additional contribution can be obtained by,

$$
\frac{d \lambda}{d \log Q^{2}} \simeq \frac{1}{16 \pi^{2}}\left[12 \lambda^{2}-\frac{12 M_{t}^{4}}{v^{4}}+\frac{3}{16}\left(2 g_{2}^{4}+\left(g^{2}+g^{1}\right)^{2}\right)\right],
$$

where the top quark Yukawa coupling is $\lambda_{t}=\sqrt{2} m_{t} / v$. The solution taking the electroweak breaking scale same as the case of Higgs self-coupling,

$$
\lambda\left(Q^{2}\right)=\lambda\left(v^{2}\right)+\frac{1}{16 \pi^{2}}\left[-\frac{12 M_{t}^{4}}{v^{4}}+\frac{3}{16}\left(2 g_{2}^{4}+\left(g_{2}^{2}+g_{1}^{2}\right)^{2}\right)\right] \log \frac{Q^{2}}{v^{2}} .
$$

If the coupling $\lambda$ is too small, the top quark contribution can be dominant and could result a negative value $\lambda\left(Q^{2}\right)<0$ which is eventually leading to a scalar potential $V\left(Q^{2}\right)<V(v)$, and it can say that the vacuum is stable since it has no minimum. 
From the stability perspective, that is, to keep $\lambda\left(Q^{2}\right)>0$, the Higgs boson mass need to be larger than a value as written in the following,

$$
M_{h}^{2}>\frac{v^{2}}{8 \pi^{2}}\left[-\frac{12 M_{t}^{4}}{v^{4}}+\frac{3}{16}\left(2 g_{2}^{4}+\left(g_{2}^{2}+g_{1}^{2}\right)^{2}\right)\right] \log \frac{Q^{2}}{v^{2}}
$$

The lower constraint on Higgs boson mass depends on the value of the energy scale $\Lambda_{c}$, if the value $\Lambda_{c} \sim 10^{3}$, the Higgs boson mass should be larger than $70 \mathrm{GeV} / c^{2}$, and if $\Lambda_{c} \sim 10^{16}$, the Higgs boson mass is larger than $130 \mathrm{GeV} / c^{2}$.

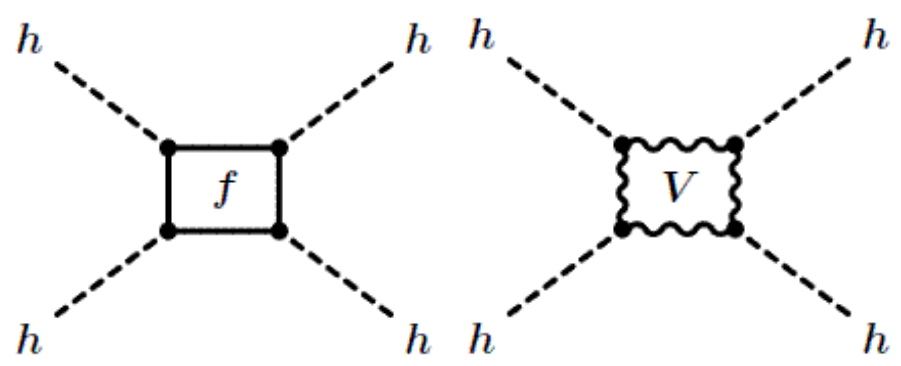

Figure 1.6: Feynman diagrams for the one-loop contribution of fermion and gauge boson to the Higgs coupling $\lambda$.

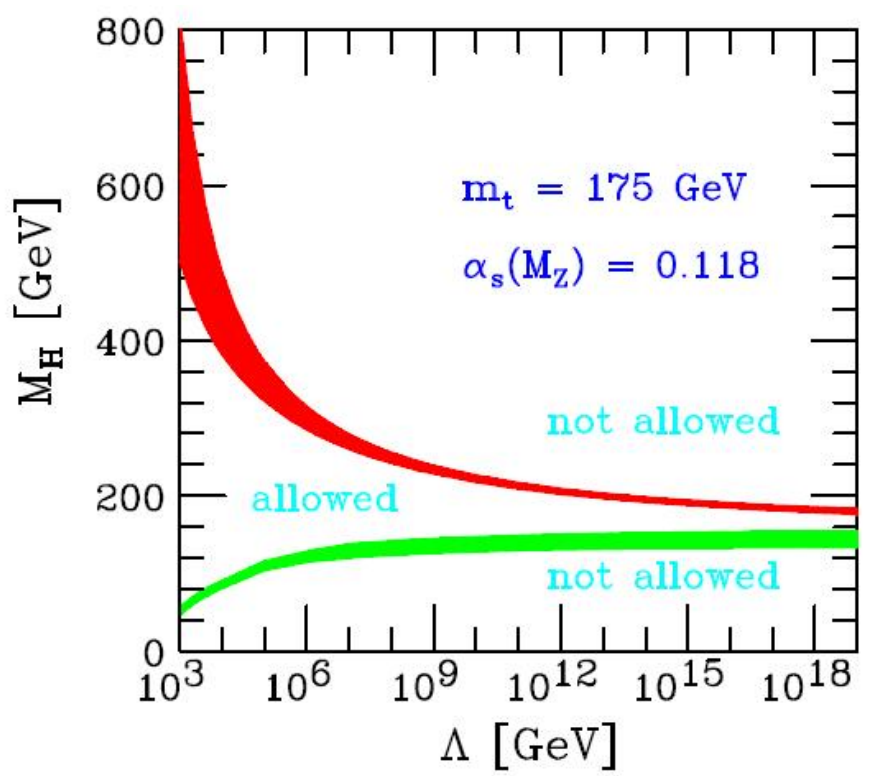

Figure 1.7: The triviality (upper) bound and the vacuum stability (lower) bound on the Higgs boson mass as a function of the cut-off scale $\Lambda$ for a top quark mass $M_{t}=175 \pm 6$ $\mathrm{GeV} / c^{2}$ and $\alpha_{s}\left(M_{Z}\right)=0.118 \pm 0.002$. 


\subsubsection{Experimental Higgs Boson Mass Constraints}

The Higgs searches have performed and are going on with the various experiments in the whole world. The results from various experiments give us the constraints on the mass of the Higgs boson, directly and indirectly. The four LEP collaborations performed the direct searches for the Higgs boson at LEP1 and LEP2 from 1989 to 2000. The collaborations reported the combined lower limits on the mass of SM Higgs boson, also set the lower limit on the mass of the fermiophobic Higgs boson. In this section, the constraints on the mass of the Higgs boson by experimental results are discussed.

\section{$\underline{\text { The Electroweak Precision Measurements }}$}

The electroweak parameters, the vector boson and top quark mass and its width, forward-backward asymmetry, and so on, are precisely measured by various experiments (ALEPH, DELPHI, L3, OPAL, SLD, CDF, DØ and NuTeV) in the world. The precise electroweak measurements leads to the constraint on the mass of the Higgs boson using the masses of the top and the Higgs boson through radiative (loop) corrections as shown in Figure 1.8. The parameter indicating the relation of the $W$ boson and the $Z$ boson mass with weak mixing angle at one loop is,

$$
\rho=\frac{M_{W}^{2}}{M_{Z}^{2}\left(1-\sin ^{2} \theta_{W}\right)} \equiv 1+\Delta r,
$$

and a radiative correction are written by,

$$
\Delta r=\frac{3 G_{F}}{8 \pi^{2} \sqrt{2}} m_{t}^{2}+\frac{\sqrt{2} G_{F}}{16 \pi^{2}} M_{W}^{2}\left[\frac{11}{3} \ln \left(\frac{M_{h}^{2}}{M_{W}^{2}}\right)+\cdots\right]+\cdots,
$$

which is quadratic in the top quark mass, while the dependence on the mass of the Higgs boson is only logarithmic, therefore the top quark mass, especially if large, is the dominant parameter in the correction to electroweak processes [14].

The electroweak precision measurements allow the constraint on the SM Higgs boson mass [15]. The Figure 1.9 shows the $\Delta \chi^{2}$ of the fit to all electroweak measurements as a function of SM Higgs Mass. From the fitting, the constraint SM Higgs mass with the experiment uncertainties are obtained as,

$$
M_{h}=84_{-26}^{+34} \mathrm{GeV} / c^{2},
$$

the shaded band represents the uncertainty due to uncalculated higher-order corrections. And the $95 \%$ one-sided confidence level upper limits on the SM Higgs mass is,

$$
M_{h}<154 \mathrm{GeV} / c^{2}
$$

when the $95 \%$ confidence level lower limits on the SM Higgs mass $114.4 \mathrm{GeV} / c^{2}$ from direct searches as discussed in the following section is included, the upper limit increases to $185 \mathrm{GeV} / c^{2}$. 


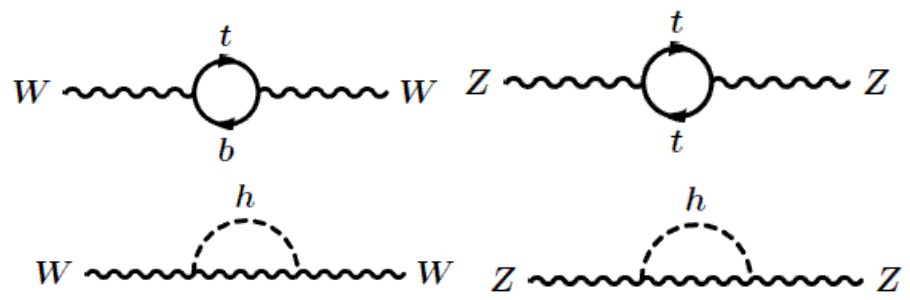

Figure 1.8: Radiative loop correction for electroweak processes.

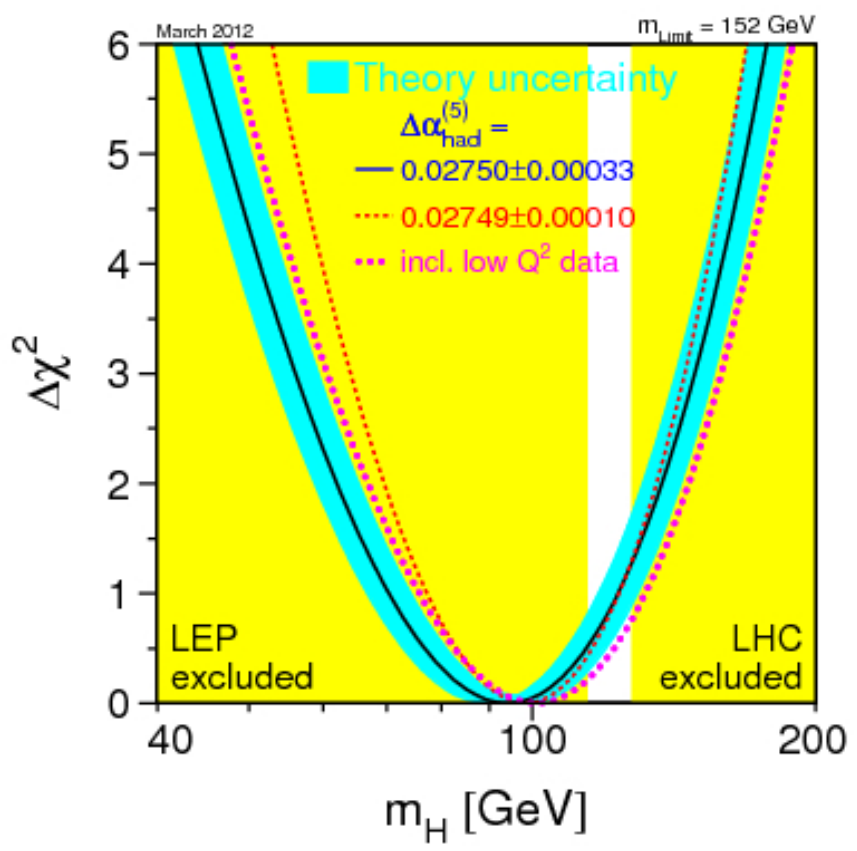

Figure 1.9: The $\Delta \chi^{2}$ of the fit to the electroweak precision data as a function of SM Higgs mass. The solid line results by including all data, and the blue band is the estimated theoretical error from missing higher-order corrections.

\section{The SM Higgs Boson Searches at LEP}

The four LEP collaborations, ALEPH, DELPHI, L3, and OPAL set a lower bound of the SM Higgs bosons at 95\% confidence level using the combined result [16]. The LEP collaborations have collected a total of $2461 \mathrm{pb}^{-1}$ of $e^{+} e^{-}$collision data at $\sqrt{s}=$ $189-209 \mathrm{GeV}$ which are used to search for the SM Higgs boson. The four results are combined and examined in a likelihood test for their consistency with two hypotheses, the background hypothesis and the signal plus background hypothesis.

The SM Higgs boson is expected to be produced mainly in association with $Z$ as $e^{+} e^{-} \rightarrow Z h$, and the SM Higgs boson is expected to decay mainly into $b \bar{b}$ pairs. The target final state are the 4 -jets event $(Z h \rightarrow q \bar{q} b \bar{b})$, the missing energy event 
$(Z h \rightarrow \nu \bar{\nu} b \bar{b})$, the leptonic event $\left(Z h \rightarrow \ell^{+} \ell^{-} b \bar{b}, \ell=e, \mu\right)$, and the tau lepton event $\left(Z h \rightarrow \tau^{+} \tau^{-} b \bar{b}\right)$.

The ratio $\mathrm{CL}_{\mathrm{s}}=\mathrm{CL}_{\mathrm{s}+\mathrm{b}} / \mathrm{CL}_{\mathrm{b}}$ is used to drive a lower bound on the $\mathrm{SM}$ Higgs boson mass, where $\mathrm{CL}_{\mathrm{s}+\mathrm{b}}$ means the compatibility for the observation and signal + background hypothesis, and $\mathrm{CL}_{\mathrm{b}}$ is the compatibility for the observation and background hypothesis. Using The quantity for setting exclusion limits by taken a mass hypothesis into account to be excluded at the $95 \%$ confidence level if the corresponding value of $\mathrm{CL}_{\mathrm{s}}$ is less than 0.05. The combined the final results from the four LEP experiments is a lower bound of $114.4 \mathrm{GeV} / c^{2}$ on the mass of the SM Higgs boson at the $95 \%$ confidence level as shown in Figure 1.10.

\section{The Fermiophobic Higgs Boson Searches at LEP}

The LEP collaborations also set a lower bound on the "benchmark" fermiophobic Higgs boson at the 95\% confidence level assuming Standard Model production rates [17]. The four experiments searched for hadronic, leptonic, and missing energy decay mode of the associated $Z$ boson in the production $Z h^{0}$ with $h^{0} \rightarrow \gamma \gamma$. For the combined data from the four experiments, the $95 \%$ confidence level lower mass limit for a benchmark fermiophobic Higgs boson is set at $109.7 \mathrm{GeV} / c^{2}$. Figure 1.11 shows the combined upper limit on $B\left(h^{0} \rightarrow \gamma \gamma\right) \times \sigma\left(e^{+} e^{-} \rightarrow h^{0} Z\right) / \sigma(\mathrm{SM})$ at $95 \%$ confidence level.

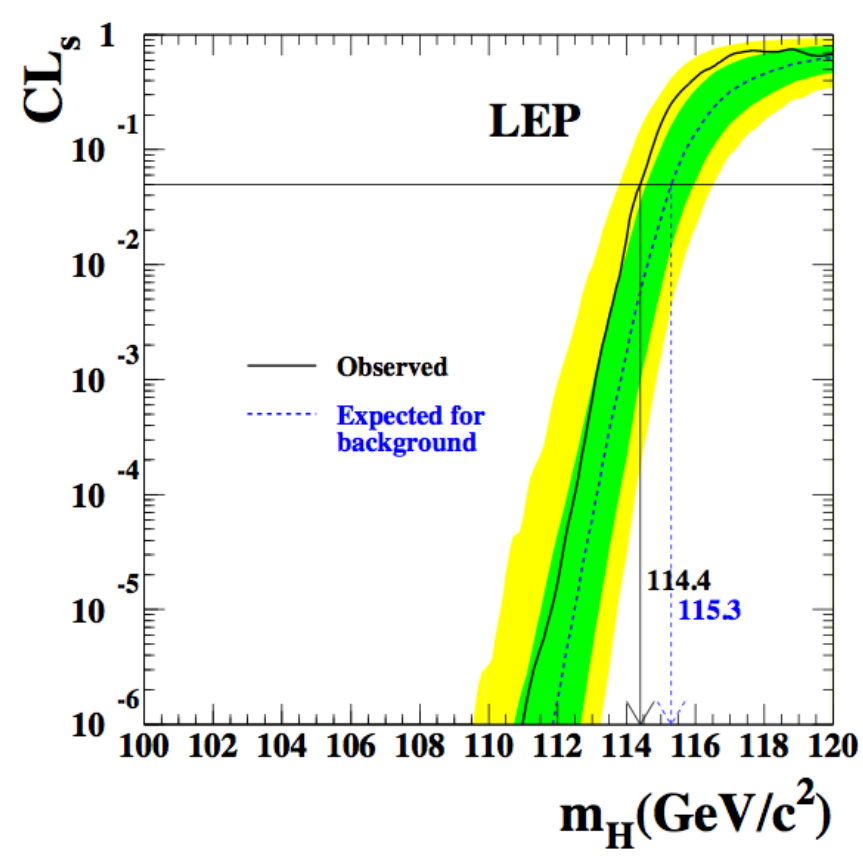

Figure 1.10: Confidence Level $\mathrm{CL}_{\mathrm{s}}$ for the signal+background hypothesis in Higgs production at LEP2. The yellow green and yellow band around the median expected line correspond to the $68 \%$ and $95 \%$ probability bands, respectively. 


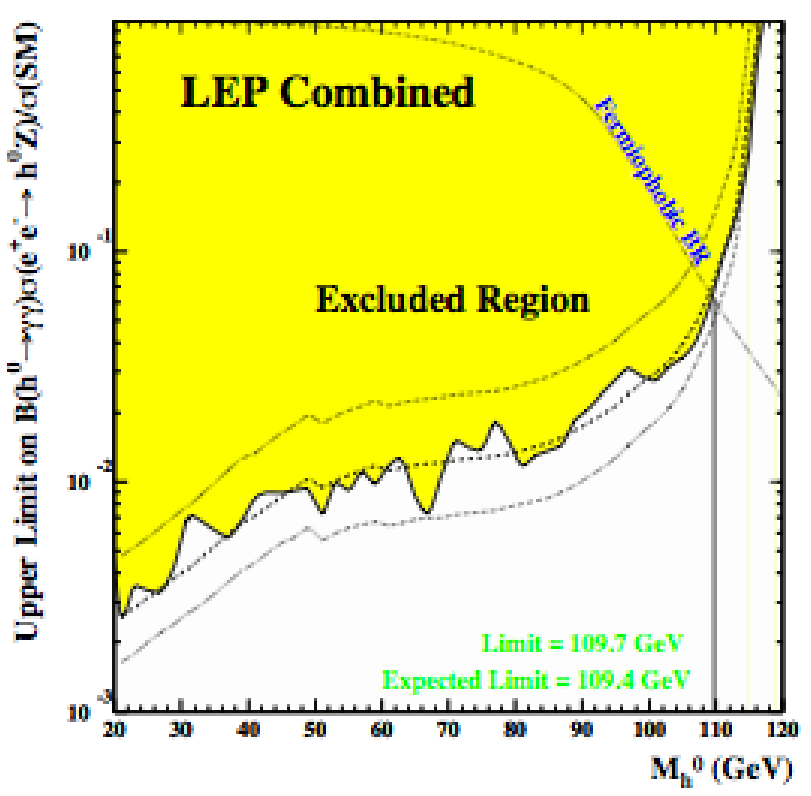

Figure 1.11: Combined LEP experiment upper limits for the Higgs bosons decaying into di-photons $B\left(h^{0} \rightarrow \gamma \gamma\right) \times \sigma\left(e^{+} e^{-} \rightarrow h^{0} Z\right) / \sigma(\mathrm{SM})$ as a function of Higgs mass at $95 \%$ confidence level.

\subsection{Higgs Boson Searches at Tevatron}

The two Tevatron collaboration, CDF and D $\varnothing$, are performing not only the SM Higgs boson searches but also the extended SM Higgs searches. The Tevatron has studied and announced many results related to the Higgs boson. In this section, the current constrains of the Higgs boson for both SM and the fermiophobic scenarios are discussed.

\section{The SM Higgs Boson Searches}

The CDF and the DØ collaborations are searching the SM Higgs boson by looking at several Higgs boson production and decay modes as described in $\S 1.2$ and $\S 1.3$. The CDF experiment searches for the SM Higgs Boson using many Higgs boson channels $[18,19,20,21,22,23,24,26,27,28,29]$. the four of them are sensitive to low mass SM Higgs $\left(M_{h}<135 \mathrm{GeV} / c^{2}\right)$ because of the looking for $H \rightarrow b \bar{b}, \tau^{+} \tau^{-}$, and $H \rightarrow \gamma \gamma$ decay mode $\left(W H \rightarrow \ell \nu b \bar{b}, Z H \rightarrow \ell \ell b \bar{b}, V H \rightarrow \nu \bar{\nu} b \bar{b}\right.$, and $H \rightarrow \tau^{+} \tau^{-}, g g \rightarrow H \rightarrow \gamma \gamma$, $\left.t \bar{t} H \rightarrow\left(b \ell \overline{\nu_{\ell}}\right)\left(\bar{b} q \bar{q}^{\prime}\right)(b \bar{b}), H q q^{\prime} \rightarrow(b \bar{b}) q q^{\prime}\right)$, while the remaining channels are sensitive to the high mass SM Higgs boson $\left(M_{h}>135 \mathrm{GeV} / c^{2}\right)$ because of the looking for $H \rightarrow W W$ $\left(g g \rightarrow H \rightarrow W W \rightarrow \ell^{ \pm} \nu \ell^{\mp} \nu, H \rightarrow Z Z \rightarrow \ell^{ \pm} \ell^{ \pm} \ell^{ \pm} \ell^{ \pm}, V H \rightarrow V W W \rightarrow \ell^{ \pm} \nu \ell^{ \pm} \nu+X\right)$, they contribute to the combined Tevatron (CDF and D $\varnothing$ ) upper limits on the SM Higgs boson production. Also the several SM Higgs search channels in the DØ experiment $[30,31,32,33,34,35,36,37,38]$ contribute to the Tevatron results.

The combination result of CDF and D $\varnothing$ Higgs boson searches is shown in Fig- 
ure 1.12 using up to $10.0 \mathrm{fb}^{-1}$ of data. The Tevatron result excluded in the mass region of the SM Higgs boson $147<m_{H}<180 \mathrm{GeV} / c^{2}$ at $95 \%$ confidence level. [39].

In 2012, we had a big progress in the Higgs studies. CDF and D $\varnothing$ announced that the evidence for a particle produced in the combination of $W H \rightarrow \ell \nu b \bar{b}, Z H \rightarrow \ell \ell b \bar{b}$, and $W H+Z H \rightarrow \operatorname{missing} E_{T}+b \bar{b}$ channels using the full Tevatron dataset, with up to 9.7 $\mathrm{fb}^{-1}$ of data analyzed [40]. An excess of data over the background is seen, which is the most significant in the mass range $120 \leq m_{H} \leq 135 \mathrm{GeV} / c^{2}$. The global significance in the range $m_{H}$ between 115 and $150 \mathrm{GeV} / c^{2}$ is 3.1 standard deviations (s.d.).

\section{The Fermiophobic Higgs Boson Searches}

The Tevatron experiments, CDF and D $\varnothing$ also search for the fermiophobic Higgs boson. The search is done by using mainly $h_{f} \rightarrow \gamma \gamma$ and $V h_{f} \rightarrow V W W \rightarrow \ell^{ \pm} \ell^{ \pm}+X$ channels. In the diphoton channel, CDF has excluded in the region of $m_{h_{f}}<114 \mathrm{GeV} / c^{2}$ [27], and DØ excluded in the region of $m_{h_{f}}<111.4 \mathrm{GeV} / c^{2}$ [41]. In this thesis, the search for the fermiophobic Higgs boson in like-sign dilepton channel is also described.

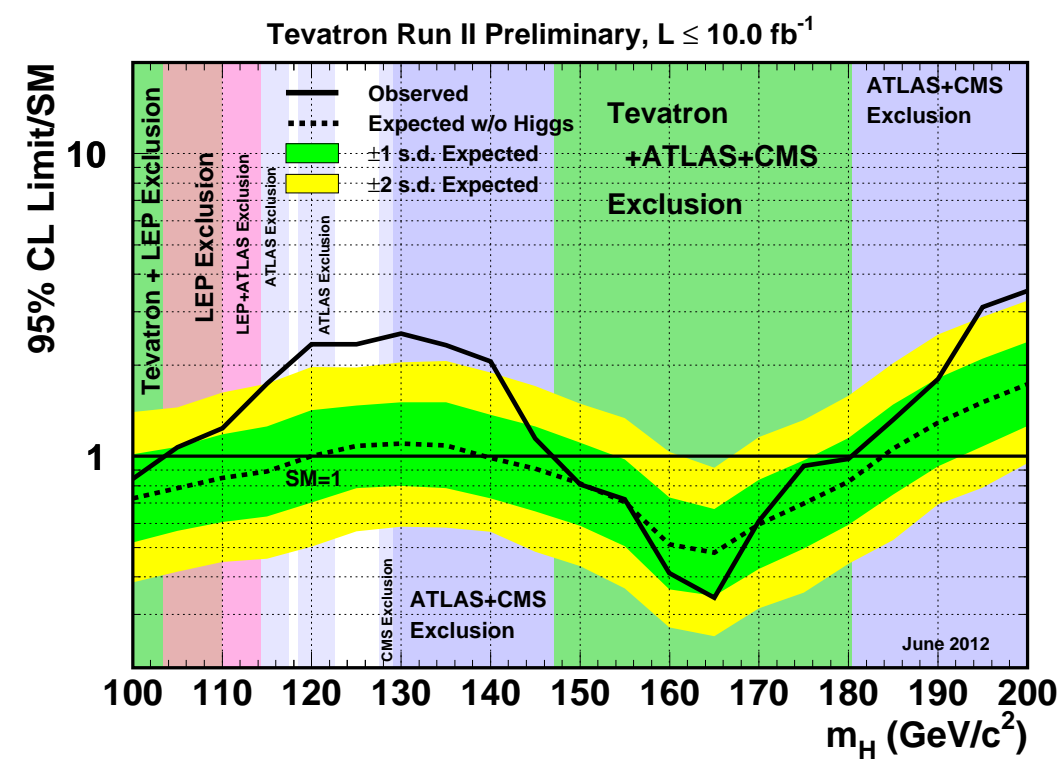

Figure 1.12: Tevatron combined upper limit on the ratios to the SM Higgs boson production as a function of the Higgs mass at 95\% confidence level. The green and yellow bands indicate the $68 \%$ and $95 \%$ probability regions, respectively.

\subsection{Observation of a New Particle at the LHC}

At the Large Hadron Collider (LHC), ATLAS and CMS made a impact statement of the observation of a new particle in the search for the standard model Higgs boson, 
respectively $[42,43]$. The mass of a new particle is around $125 \mathrm{GeV} / c^{2}$ and compatible with the production and decay of the standard model Higgs boson. Furthermore, both experiments constrained the mass of the Higgs boson strongly. ATLAS excluded 111$122 \mathrm{GeV} / c^{2}$ and $131-559 \mathrm{GeV} / c^{2}$, and CMS excluded 110-122.5, 127-600 GeV/c $c^{2}$ at 95\% confidence level, respectively. Both results are shown in Figures 1.13 and 1.14. However, if it is the Higgs boson, we have to investigate by looking at various production and decay channels and the nature of the particle (e.g. spin 0 or not).

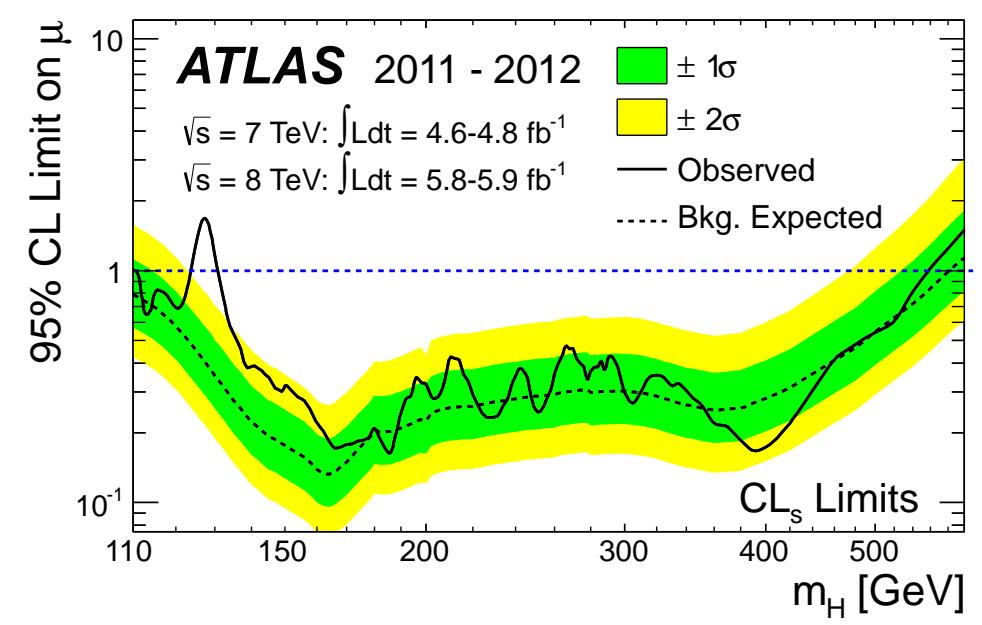

Figure 1.13: ATLAS combined upper limit on the ratios to the SM Higgs boson production as a function of the Higgs mass at 95\% confidence level. The green and yellow bands indicate the $68 \%$ and $95 \%$ probability regions, respectively. 


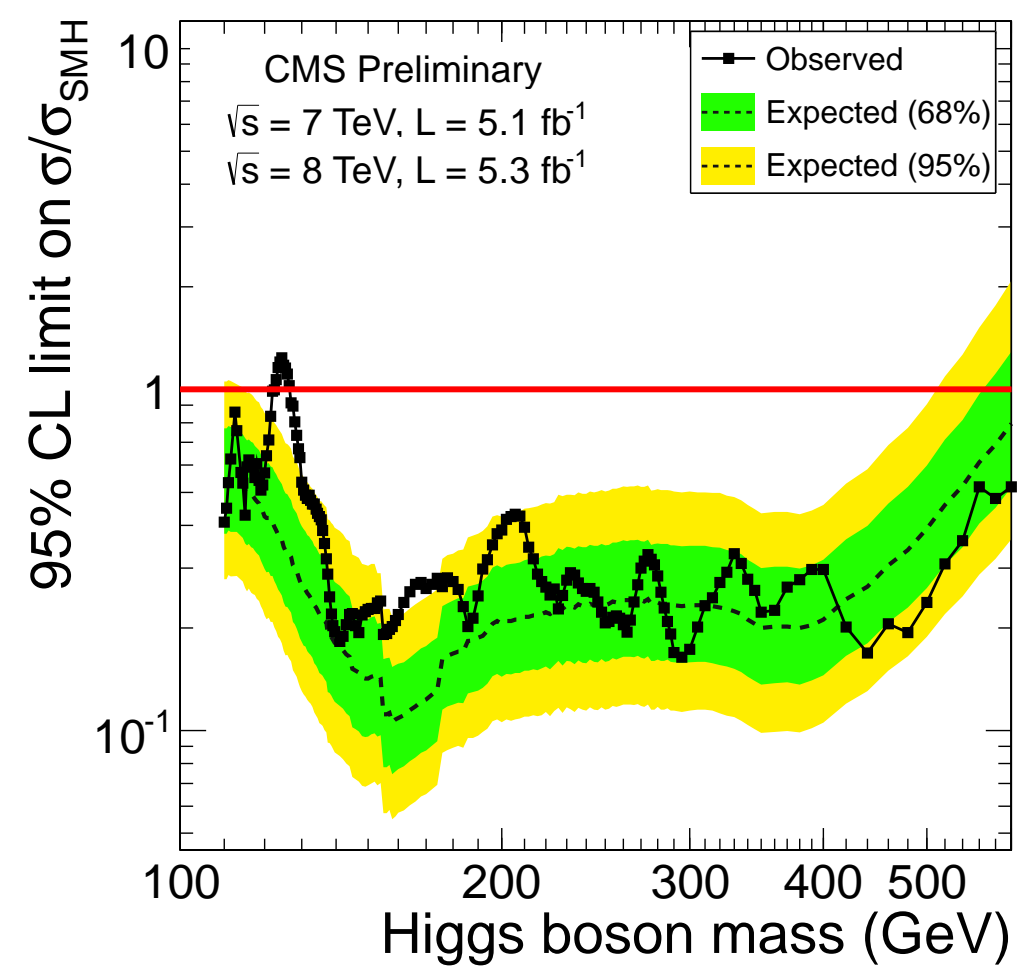

Figure 1.14: CMS combined upper limit on the ratios to the SM Higgs boson production as a function of the Higgs mass at 95\% confidence level. The green and yellow bands indicate the $68 \%$ and $95 \%$ probability regions, respectively. 



\section{Chapter 2}

\section{Experimental Apparatus}

The Tevatron Collider is one of the high energy frontier accelerators in particle physics. The Tevatron is located at Fermilab in Batavia, Illinois USA. It is currently providing highest energy proton-antiproton collisions with $\sqrt{s}=1.96 \mathrm{TeV}$. The collisions occur at two points in Tevatron ring which has a radius of about $1 \mathrm{~km}$. In each collision point, a detector is instrumented: One is the Collider Detector at Fermilab (CDF II) and the other is DØ. This study uses data collected with the CDF II. The CDF II is a general purpose solenoidal detector which combines precision charged particle tracking, scintillator based calorimetry, and muon detection chambers and scintillators. This chapter describes the beam production and acceleration system, and the CDF II detector design.

\subsection{The Accelerator Complex}

The Tevatron is a chain of accelerators that gradually increase the energy of protons and antiprotons. The protons are abundant and readily in nature, while antiprotons must be produced and stored. In addition, a single accelerator cannot bring particles from rest to very high energies because no magnets have the dynamic range necessary. Consideration of these requirements led to the design of a chain of accelerators at Fermilab. Figure 2.1 shows a diagram and aerial photograph of the chain of the Fermilab accelerator chain.

\subsubsection{Proton Production and Boosting}

The Cockcroft-Walton pre-accelerator provides the first stage of the acceleration. The accelerator ionizes the hydrogen gas to $\mathrm{H}^{+}$ions, which are accelerated to $750 \mathrm{keV}$ of kinetic energy.

The ionized hydrogen gases $\left(\mathrm{H}^{+}\right)$enter a liner accelerator (Linac), approximately $150 \mathrm{~m}$ long, and the ions are accelerated to $400 \mathrm{MeV}$. The acceleration in the Linac is done by a series of "kicks" from Radio Frequency (RF) cavities. 

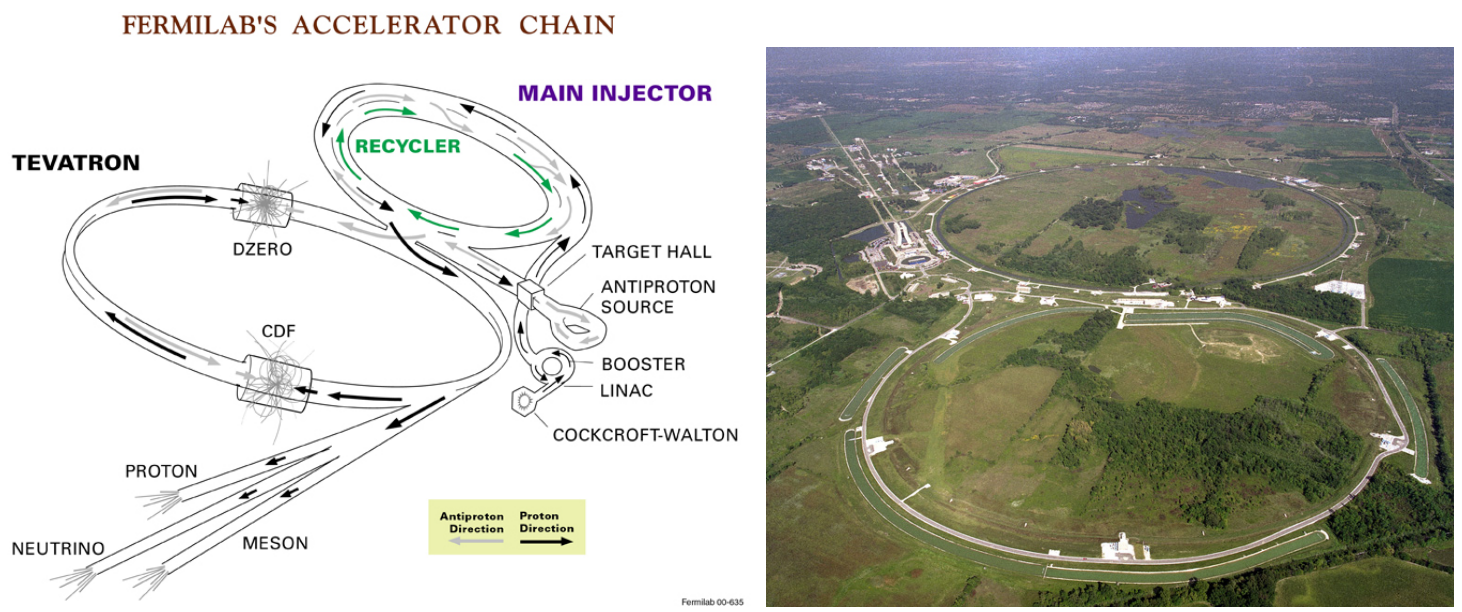

Figure 2.1: A diagram (left) and aerial photograph (right) of the Fermilab accelerator chain.

The $\mathrm{H}^{+}$ions with $400 \mathrm{MeV}$ are injected into the Booster. The Booster is a circular synchrotron, approximately $150 \mathrm{~m}$ in diameter. A carbon foil strips the electrons from the $\mathrm{H}^{+}$ions, leaving behind protons. The intensity of the proton beam is increased by injecting new protons into the same orbits as the circulating ones. The protons are accelerated from $400 \mathrm{MeV}$ to $8 \mathrm{GeV}$ with a series of magnets arranged around a $75 \mathrm{~m}$ radius circle, with $18 \mathrm{RF}$ cavities interspersed.

\subsubsection{Main Injector}

The Main Injector (MI) is a circular synchrotron seven times the circumference of the Booster and slightly more than half the circumference of the Tevatron. Main Injector has 18 accelerating cavities. It can accelerate $8 \mathrm{GeV}$ protons from the Booster to either $120 \mathrm{GeV}$ or $150 \mathrm{GeV}$, depending on their destination. When used to stack antiprotons, the final energy is $120 \mathrm{GeV}$. When used to inject into the Tevatron, the final beam energy is $150 \mathrm{GeV}$. As well as accepting protons from Booster, the Main Injector can accept antiprotons from the Antiproton Source. The Main Injector can accelerate beam as fast as every 2.2 seconds.

\subsubsection{Antiproton Source}

In order to produce antiprotons, the protons with $120 \mathrm{GeV}$ are extracted from the MI and strike a nickel target at the Antiproton source. These high-energy protons striking the target produce a spray of all sorts of secondary particles. Using magnets to choose which momentum and charge we can collect $8 \mathrm{GeV}$ antiprotons from this spray. Approximately one antiproton is produced per $10^{5}$ protons. These antiprotons are directed into the Debuncher. 
The Debuncher is a rounded triangular-shaped synchrotron with a mean radius of $90 \mathrm{~m}$. It can accept $8 \mathrm{GeV}$ antiprotons from the target station, and maintain the beam at a energy of $8 \mathrm{GeV}$. Its primary purpose is to efficiently capture the high momentum spread antiprotons coming from the target using a RF manipulation called bunch rotation which reduce the antiproton momentum spread. The reduction is done to improve the Debuncher to Accumulator transfer because of the limited momentum aperture of the Accumulator at injection.

The Accumulator is also triangular-shaped synchrotron and is mounted in the same tunnel as the Debuncher. It is the storage ring for antiprotons, all of the antiprotons made are stored here at $8 \mathrm{GeV}$ and cooled until need.

\subsubsection{Recycler}

The Recycler is an antiproton storage ring installed in the same tunnel as the MI. The proposed purpose of the Recycler was to recycle the antiproton from a Tevatron store, cooling them and storing them alongside those sent from the Antiproton Source. This was abandoned after early problems in RunII. The Recycler now accepts transfers only from the Antiproton Source and cools them further than the antiprotons Accumulator is capable. The Recycler uses both a stochastic cooling system and an electron cooling system. Stochastic cooling is used to cool the beam in Recycler, but loses its effectiveness with higher intensities. Once above $2 \times 10^{12}$ antiprotons in the Recycler, electron cooling is required. Electron cooling works on the principle of momentum transfer between electrons and antiprotons, a highly concentrated, cool beam of electrons is driven at the same energy as the antiprotons and laid overtop of the antiprotons. The resulting glancing collisions between electrons and antiprotons transfer some of the momentum from the "hot" antiprotons to the "cool" electrons. With enough electrons, a substantial longitudinal cooling force is produced by absorbing momenta from the antiprotons allowing for more compact, brighter bunches to send to the Tevatron.

\subsubsection{Tevatron}

The Tevatron is the largest part of the Fermilab accelerators, with a circumference of approximately $6 \mathrm{~km}$ long. It is a circular synchrotron with eight accelerating cavities. The Tevatron can accept both protons and antiprotons from MI and accelerate them from $150 \mathrm{GeV}$ to $980 \mathrm{GeV}$. In Collider mode, the Tevatron can store beam for hours at a time. Because the Tevatron is a primarily storage ring, the length of time between acceleration cycles is widely variable. The Tevatron is the cryogenically cooled accelerator. The magnets used in the Tevatron are made up of a superconducting niobium/titanium alloy that needs to be kept extremely cold $(\sim 4 \mathrm{~K})$ to remain a superconductor. The benefit of having superconducting magnets is the increased magnetic fields possible when high currents can be run through thin wires without fear of damage related to excessive resistive heating. 


\subsubsection{Luminosity}

The luminosity of collisions can be expressed as:

$$
\mathcal{L}=\frac{f N_{B} N_{p} N_{\bar{p}}}{2 \pi\left(\sigma_{p}^{2}+\sigma_{\bar{p}}^{2}\right)} F\left(\frac{\sigma_{l}}{\beta^{*}}\right)
$$

where $f$ is the revolution frequency, $N_{B}$ is the number of bunched, $N_{p(\bar{p})}$ is the number of protons (antiprotons) per bunch, and $\sigma_{p(\bar{p})}$ is the protrons (antiprotons) RMS beam size at the interaction point. $F$ is a form factor which corrects for the bunch shape and depends on the ratio of $\sigma_{l}$, the bunch length to $\beta^{*}$, the beta function, at the interaction point. The beta function is a measure of the beam width, and it is proportional to the beam's $x$ and $y$ extent in phase space. Table 2.1 shows the accelerator parameter in the current run (Run II). The current peak luminosity is $\sim 4.4 \times 10^{32} \mathrm{~cm}^{-2} \mathrm{~s}^{-1}$. The delivered luminosity is $12 \mathrm{fb}^{-1}$ and actual recorded luminosity is $10 \mathrm{fb}^{-1}$, which is collected between February 2002 and September 2011. Figure 2.2 shows the integrated luminosity measured by CDF.

\begin{tabular}{lc}
\hline \hline Parameter & Run II \\
\hline Number of bunhes $\left(N_{B}\right)$ & 36 \\
Bunch length $[\mathrm{m}]$ & 0.37 \\
Bunch spacing [ns] & 396 \\
Protons/bunch $\left(N_{p}\right)$ & $2.7 \times 10^{11}$ \\
Antiprotons/bunch $\left(N_{\bar{p}}\right)$ & $3.0 \times 10^{10}$ \\
Total antiprotons & $1.1 \times 10^{12}$ \\
$\beta^{*}[\mathrm{~cm}]$ & 35 \\
Interactions/crossing & 2.3 \\
\hline \hline
\end{tabular}

Table 2.1: Accelerator parameters for Run II configurations.

\subsection{The Collider Detector at Fermilab}

The CDF II detector [44] is a general purpose solenoidal detector which combines precision charged particle tracking with the fast projective calorimetry and fine grained muon detection. Figure 2.3 and Figure 2.4 show a cut away view and elevation view of the CDFII detector for each. Tracking systems are made up Silicon Trackers, Central Outer Tracker (COT), and Superconducting Solenoid which measures precise trajectories and momenta of charged particles and reconstruct vertices. The solenoid surrounded the Silicon Trackers and COT, has $1.5 \mathrm{~m}$ in radius and $4.8 \mathrm{~m}$ long, and generates a $1.4 \mathrm{~T}$ magnetic field parallel to the beam axis. Calorimetry Systems measure the energy of particles. Muon Chambers detect the particles penetrating both Tracking Systems and Calorimetry Systems. Muons deposit small amount of ionization energy 
Yeฉf002 $20032004200800 Q 00720082009$

Month 47101471047147071010147

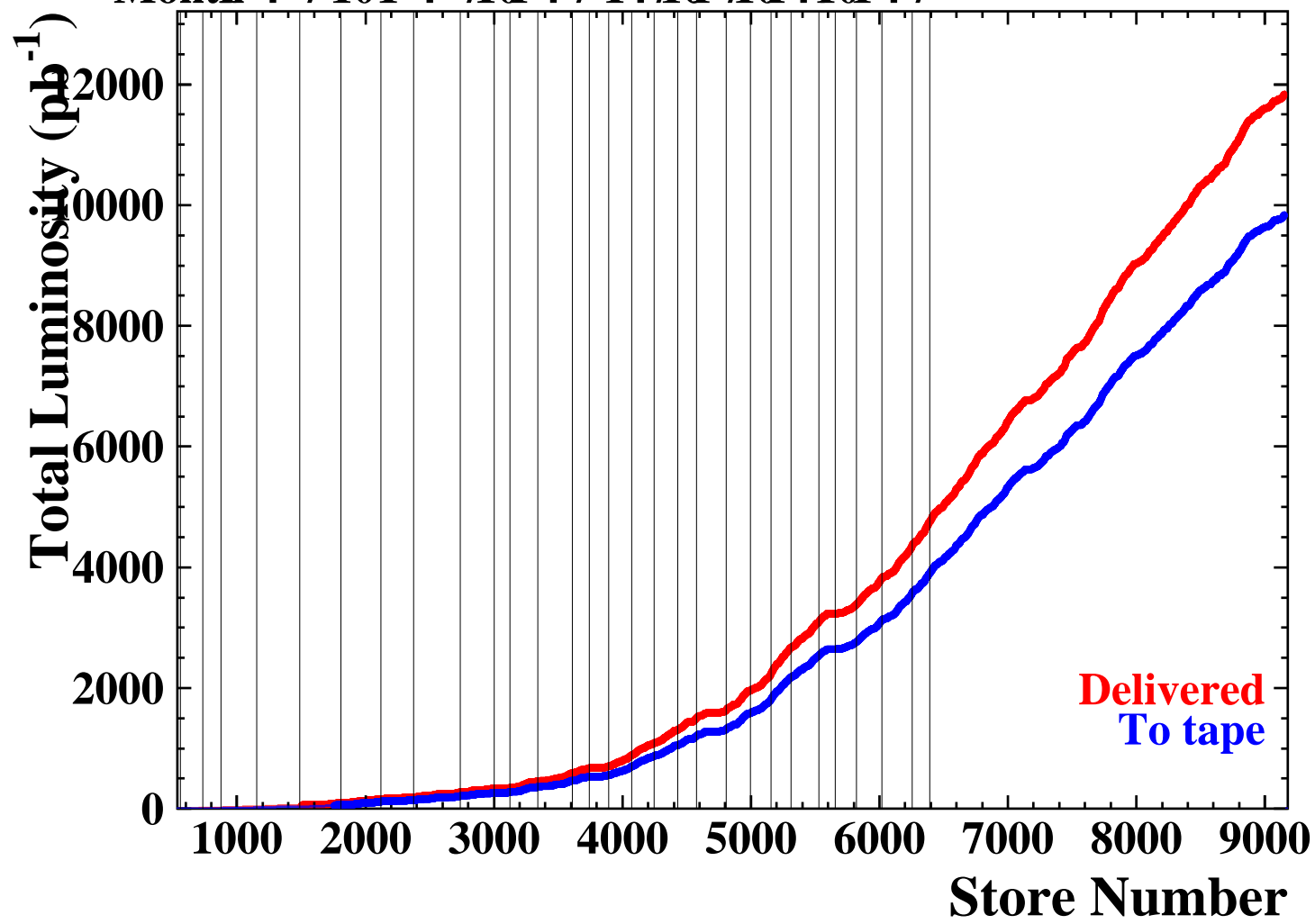

Figure 2.2: Integrated luminosity as a function of store number between February 2002 and September 2011.

in the material because they act as minimally ionizing particles (MIP), that is, the penetrating particles are mostly muons.

\subsection{Coordinate System in the CDF}

The standard coordinate system used in the CDF is the right-handed coordinate system. The $z$-axis is oriented to the direction of the proton beam. The $x$-axis points horizontally away from the detector and the $y$-axis is vertical pointing up-wards. It is helpful to use the cylindrical coordinate. The azimuthal angle $\phi$ is $x-y$ plane angle around the beam line. The polar angle $\theta$ is measured starting from the $z$-axis. The rapidity of a particle is defined as,

$$
y \equiv \frac{1}{2} \ln \left(\frac{E+p_{z}}{E-p_{z}}\right)
$$




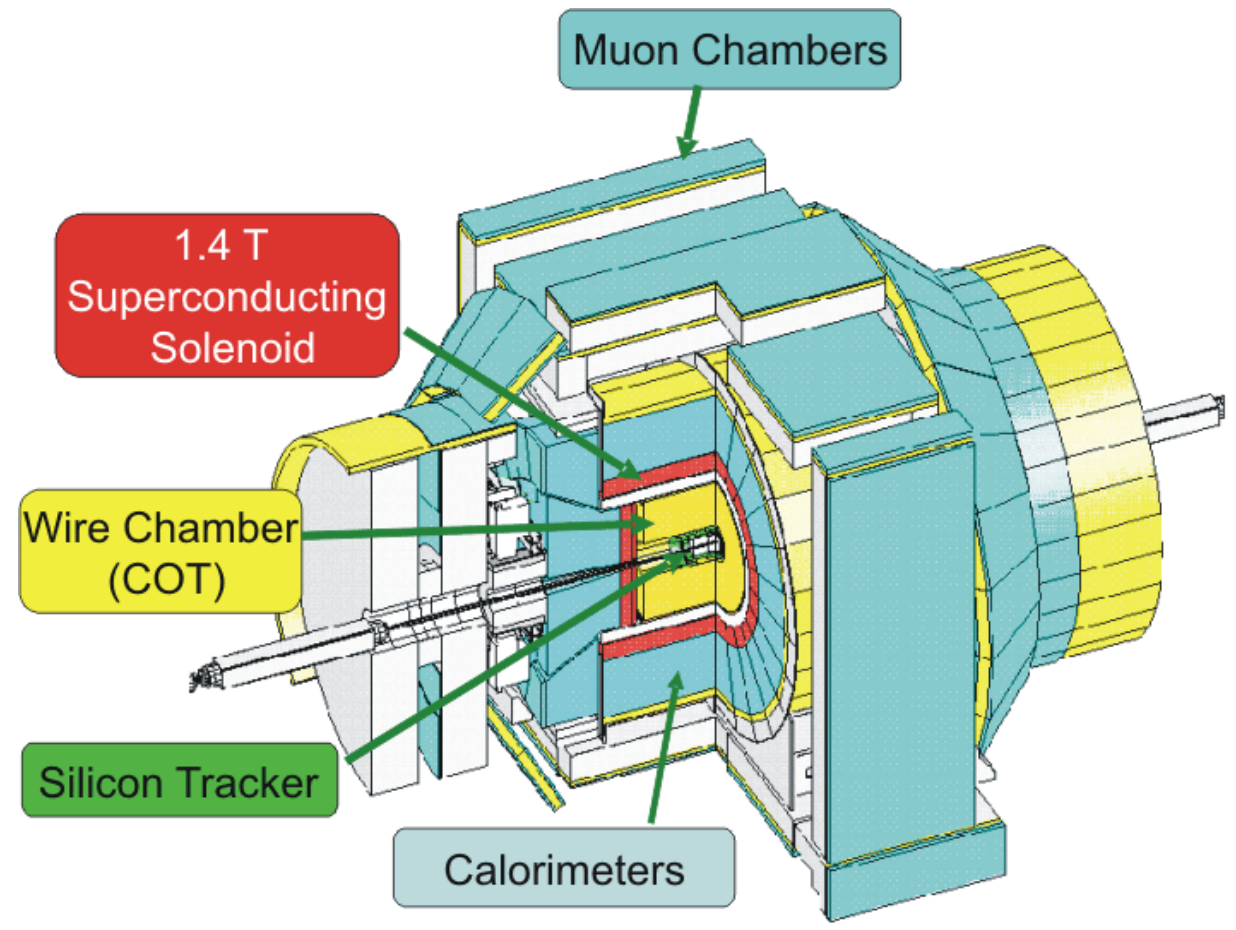

Figure 2.3: Cut away view of the CDF II detector.

where $E$ is the energy of the particle and $p_{z}$ is its longitudinal momentum. For highly boosted particles, $E \sim p$ and $p_{z}=p \cos \theta$, that is, the rapidity can be approximated by pseudorapidity,

$$
\eta=-\ln \left(\tan \frac{\theta}{2}\right)
$$

\subsection{Tracking Systems}

For CDF analysis technique, precision charged particle tracking is very important. CDF II detector has an open cell drift chamber, the Central Outer Tracker (COT) covers the region $|\eta| \leq 1.0$. Inside the COT, a silicon "inner tracker" is built from three components. Layer 00 (L00) is mounted on the beam pipe, very close to the beam line. Its primary purpose is to improve the impact parameter resolution. A micro-vertex detector at very small radii, so-called Silicon Vertex Detector (SVX-II), establishes the ultimate impact parameter resolution. Two additional silicon layers at intermediate radii, so-called Intermediate Silicon Layers (ISL), provides $p_{T}$ resolution and b-tagging in the forward region $1.0 \leq|\eta| \leq 2.0$, and stand-alone silicon tracking over the full region $|\eta| \leq 2.0$. The stand-alone silicon segments allow integrated tracking algorithms which maximize tracking performance over the whole region $\eta \leq 2.0$. In the central region $(\eta \leq 1.0)$, the stand-alone silicon segment can be linked to the fill 


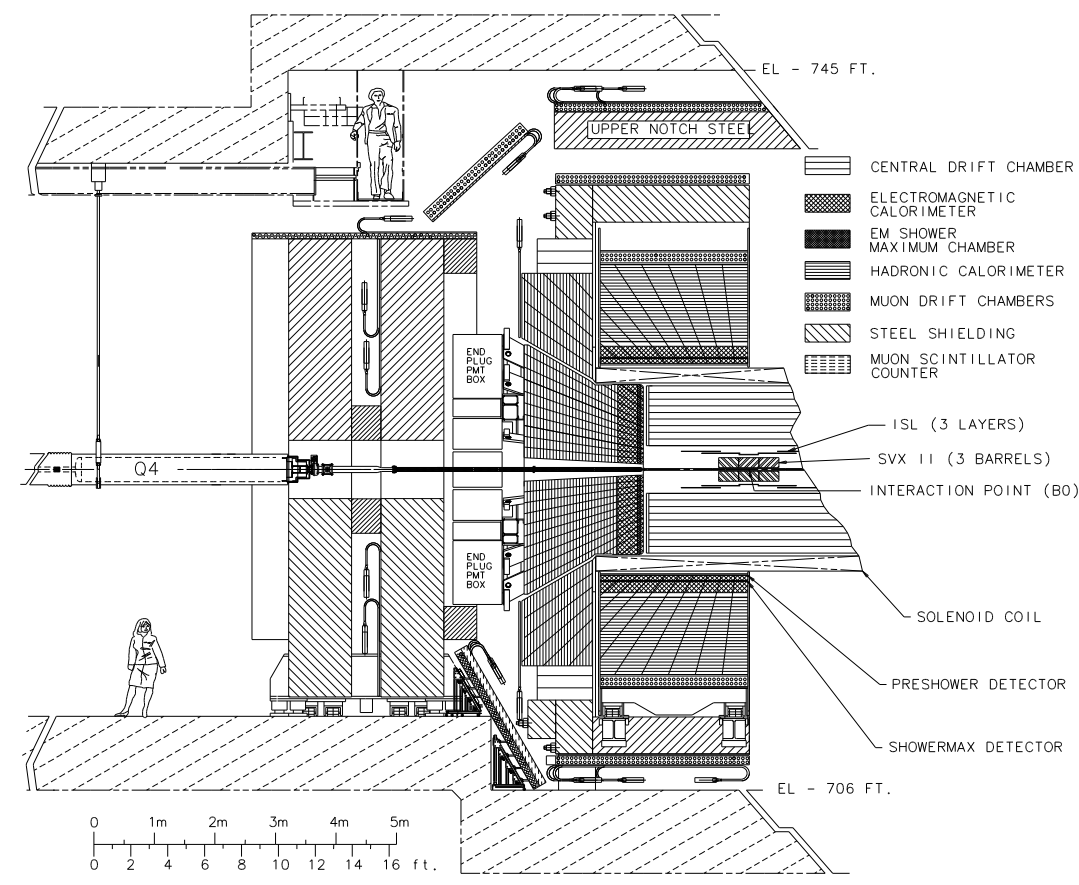

Figure 2.4: Elevation view of the CDF II detector.

COT track to give excellent $p_{T}$ and impact parameter resolution.

\subsubsection{Layer 00}

Layer $00[45,46]$ is installed directly in the beam pipe. L00 was added at beginning of RunII for two reasons. As the first reason, it is to improve the impact parameter resolution of the CDF detector. Placement of a minimal material silicon layer at a smaller radius provides a precise measurement. Secondly, L00 was installed to extend the useful lifetime of the silicon system. The inner layers SVX-II will have a limited lifetime due to radiation damage. The design has six narrow (128 channels) and six wide (256 channels) groups in $\theta$ at $r=1.35 \mathrm{~cm}$ and $r=1.62 \mathrm{~cm}$ respectively. There are six readout modules in $z$, with two sensors bounded together in each module for a total length of $95 \mathrm{~cm}$. The sensors are single-sided $p$-in- $n$ silicon with a $25(50) \mu \mathrm{m}$ implant(readout) pitch. These have been produced by Hamamatsu Photonics (HPK), SGS-Thompson (ST) and Micron. These sensors can be biased up to 500V, limited by the maximum range of the power supplies. Figure 2.5 shows the end view of L00 and a part of SVX-II (L0 and L1).

\subsubsection{Silicon Vertex Detector}

Silicon Vertex Detector (SVX, SVX-II) [47] is the core detector for the silicon tracking and for the trigger on tracks with a large impact parameter with respect to an interac- 


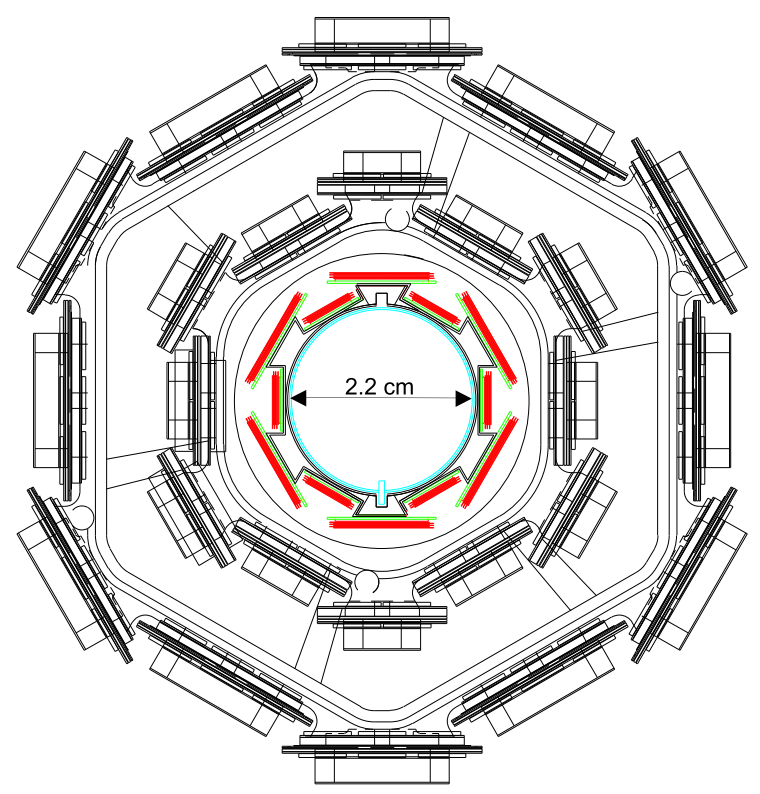

Figure 2.5: End view of Layer 00 (colored), also showing a part of SVX-II (un-colored).

tion point [48]. The SVX-II detector has 5 layers of double-sided sensors surround the L00 at radii from 2.5 to $10.6 \mathrm{~cm}$. Three layers (L0, L1, and L3) are made of Hamamatsu silicon with the $n$ strips perpendicular to the $p$ strips. The remaining two layers (L2 and L4) are Micron sensors with a stereo angle of $1.2^{\circ}$ between the $n$ and $p$ strips. The strip pitch varies between 60 to $140 \mu \mathrm{m}$, depending on the layer radius. The maximum bias voltages that can be applied to Hamamatsu and Micron sensors are $170 \mathrm{~V}$ and 70 $\mathrm{V}$ respectively, limited by the breakdown voltage of the integrated coupling capacitors and subtle sensor effects. The SVX-II can provide track information to $|\eta|<2.0$. Table 2.2 shows the design parameters of the SVX-II. Figure 2.6 shows $3 \mathrm{D}$ view and $r-\phi$ view for SVX-II.

\subsubsection{Intermediate Silicon Layers}

Intermediate Silicon Layers (ISL) $[49,50]$ provides an extended forward coverage and links tracks between the COT and the SVX-II, and also can provide stand-alone 3D track information in the forward region. The ISL detector has one central layer at radius of $22 \mathrm{~cm}$ covering $|\eta|<1.0$, and two forward layers at radii of $22 \mathrm{~cm}$ and $28 \mathrm{~cm}$ covering $1<|\eta|<2$, with total length of $3 \mathrm{~m}$. It is made of double-sided silicon with strips at a stereo angle of $1.2^{\circ}$, and a strip of $112 \mu \mathrm{m}$. The breakdown voltage of the sensors is $100 \mathrm{~V}$ limited by the breakdown voltage of the coupling capacitors. 


\begin{tabular}{lccccc}
\hline \hline Parameter & $\begin{array}{c}\text { Layer 0 } \\
(\mathrm{L} 0)\end{array}$ & $\begin{array}{c}\text { Layer 1 } \\
(\mathrm{L} 1)\end{array}$ & $\begin{array}{c}\text { Layer 2 } \\
(\mathrm{L} 2)\end{array}$ & $\begin{array}{c}\text { Layer 3 } \\
(\mathrm{L} 3)\end{array}$ & $\begin{array}{c}\text { Layer 4 } \\
(\mathrm{L} 4)\end{array}$ \\
\hline Number of $\phi$ strips & 256 & 384 & 640 & 768 & 896 \\
Number of $z$ strips & 512 & 576 & 640 & 512 & 896 \\
stereo angle (degree) & 90 & 90 & +1.2 & 90 & -1.2 \\
$\phi$ strip pitch $[\mu \mathrm{m}]$ & 60 & 62 & 60 & 60 & 65 \\
$z$ strip pitch [ $\mu \mathrm{m}]$ & 141 & 125.5 & 60 & 141 & 65 \\
Total width [mm] & 171.140 & 25.594 & 40.300 & 47.860 & 60.170 \\
Total length [mm] & 74.3 & 74.3 & 74.3 & 74.3 & 74.3 \\
Active width [mm] & 15.300 & 23.746 & 38.340 & 46.020 & 58.175 \\
Active length [mm] & 72.43 & 72.43 & 72.38 & 72.43 & 72.38 \\
Number of sensors & 144 & 144 & 144 & 144 & 144 \\
\hline \hline
\end{tabular}

Table 2.2: Design parameters of the Silicon Vertex Detector.
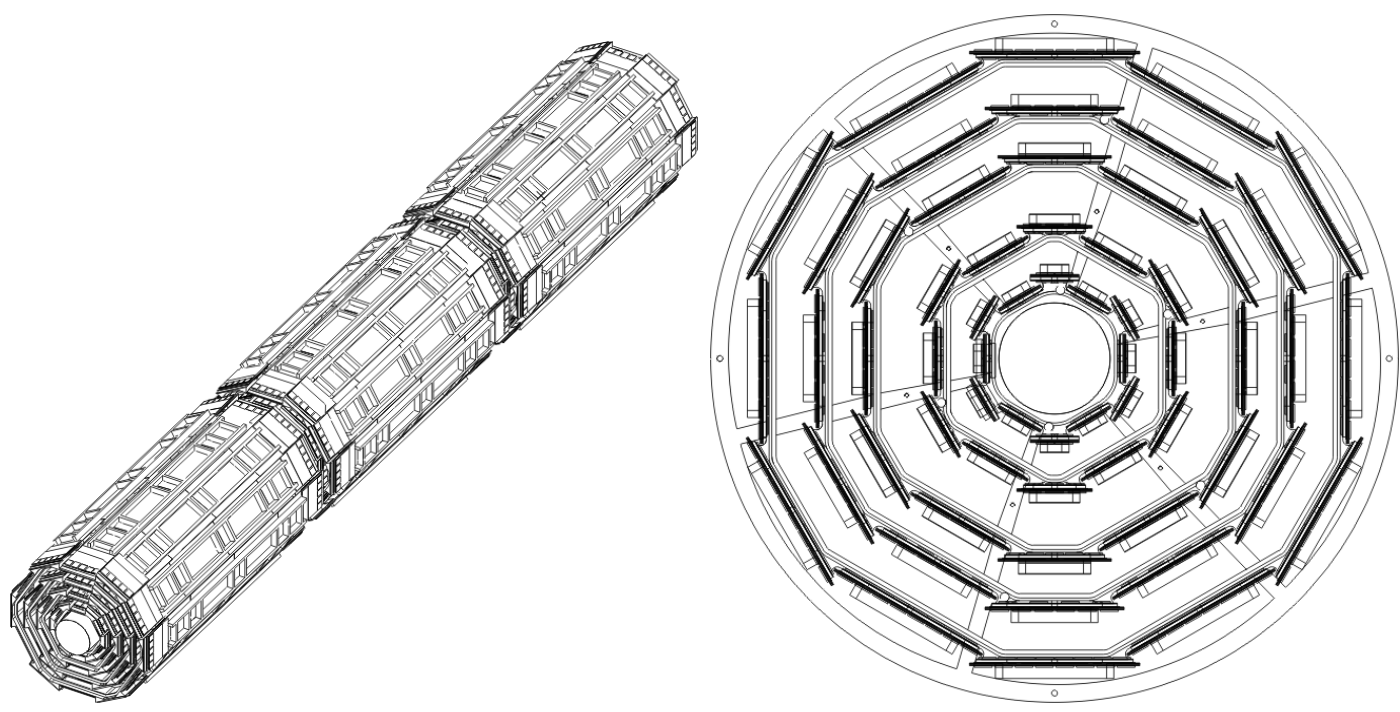

Figure 2.6: 3D view of the three barrels (left) and $r-\phi$ view of the barrel showing the 12 wedges with the 5 layers.

\subsubsection{Central Outer Tracker}

The Central Outer Tracker (COT) [51] is a cylindrical open-cell drift chamber spanning from 44 to $132 \mathrm{~cm}$ in radii, and $310 \mathrm{~cm}$ long. It operates inside a 1.4 Tesla solenoidal magnetic field and is designed to find charged tracks in the region $|\eta| \leq 1.0$. The resolution of a hit position is approximately $140 \mu \mathrm{m}$ and the momentum resolution $\sigma\left(p_{T}\right)$ $=0.0015(\mathrm{GeV} / c)^{-1}$. The COT is segmented into 8 super-layers alternating stereo and axial, with a stereo angle of $\pm 2^{\circ}$. Each super-layer contains 12 sense wires alternated with 13 potential wires which provide the field shaping within the cell yielding a total 


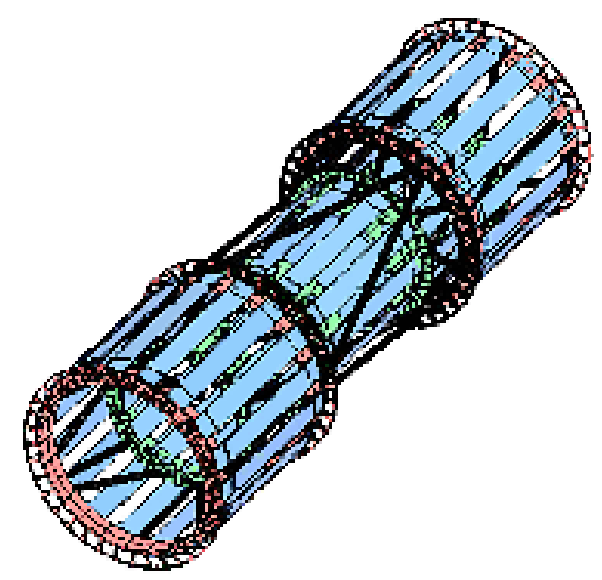

Figure 2.7: 3D veiw of the ISL spaceframe.

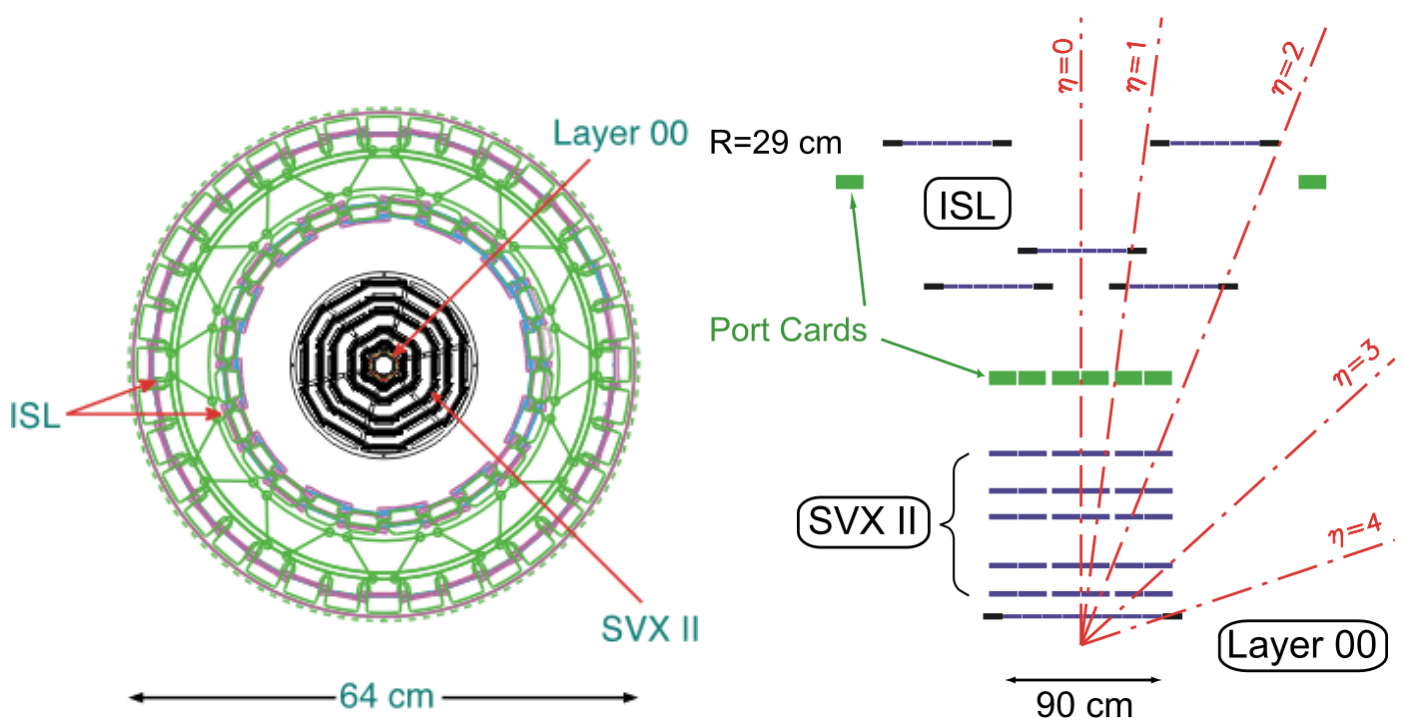

Figure 2.8: $r-\phi$ veiw (left) and $r-z$ view (right) of the silicon detectors.

of 96 measurement layers. For the entire cell chamber, there are 30,240 sense wires and 32,760 potential wires. Operating with an Argon-Ethane (50:50) gas mixture the maximum drift time is approximately $180 \mathrm{~ns}$. The cells ate tilted at $35^{\circ}$ to account for the Lorentz angle such that the drift direction is azimuthal. Tracks originating from the interaction point which have $|\eta|<1$ pass through all 8 superlayers of the COT. Tracks which have $|\eta|<1.3$ pass through 4 or more superlayers. Table 2.3 shows a mechanical summary of the COT. Figure 2.9 shows the cell layout for super-layer 2 (SL2). Figure 2.10 shows the east endplate slots sense and field planes. 


\begin{tabular}{lr}
\hline \hline Parameter & $(50: 50)$ \\
\hline Gas (Argon:Ethane) & 96 \\
Number of Layers & 8 \\
Number of Super-layers & $+2,0,-2,0,+2,0,-2,0$ \\
Stereo Angle (degree) & $168,192,240,288,336,384,432,480$ \\
Cells/Layers & $12,12,12,12,12,12,12,12$ \\
Sense Wires/Cell & $46,58,70,82,94,106,117,129$ \\
Radius at Center of SL (cm) & $35^{\circ}$ \\
Tilt Angle & $1.6 \% X_{0}$ \\
Material Thickness & $1.9 \mathrm{kV} / \mathrm{cm}$ \\
Drift Field & $0.88 \mathrm{~cm}$ \\
Maximum Drift Distance & $177 \mathrm{~ns}$ \\
Maximun Drift Time & 30,240 \\
Number of Channels & \\
\hline \hline
\end{tabular}

Table 2.3: Design parameters of the Central Outer Tracker.

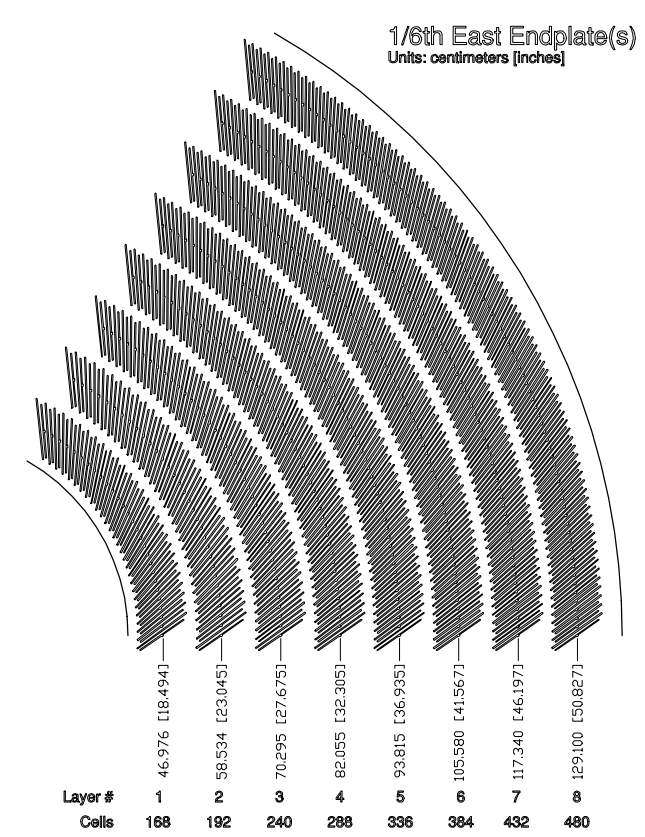

Figure 2.9: East endplate slots sense and field planes are at the clock-wise edge of each slot.

\subsection{Calorimeter Systems}

Segmented electromagnetic and hadron sampling calorimeters surround the tracking system and measure the energy flow of interacting particles in the region of $|\eta|<3.6$. 


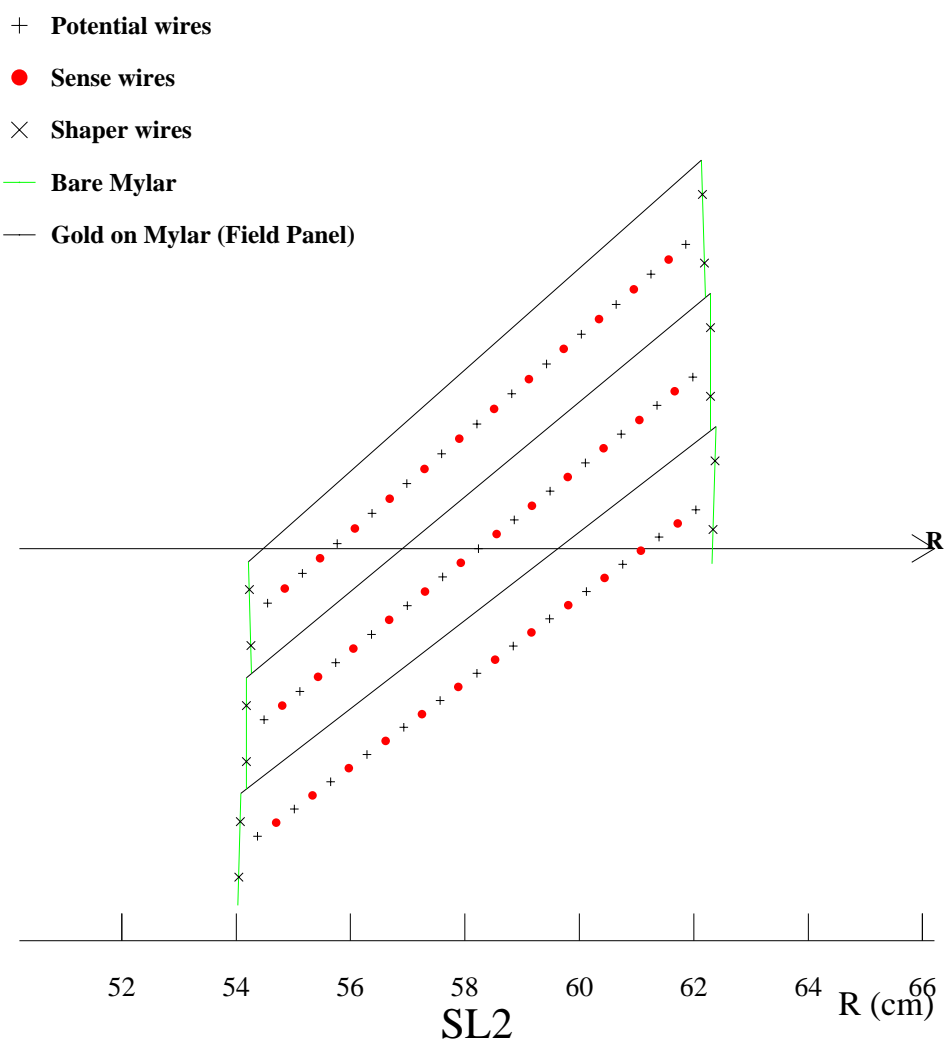

Figure 2.10: Nominal cell layout for SL2.

The calorimeter systems are divided into 2 systems with respect to the pseudo-rapidity range, central and plug(forward) region. The Central Electromagnetic Calorimeter (CEM) covers the $|\eta|<1.1$, which uses lead sheets interspersed with polystyrene scintillator as the active medium and employs phototube readout. The Central Hadronic Calorimeter (CHA) covers the region of $|\eta|<0.9$, which uses steel absorber interspersed with acrylic scintillator as the active medium. The plug calorimeters, Plug Electromagnetic Calorimeter (PEM) and Plug hadron calorimeter (PHA), cover the $1.1<|\eta|<3.6$. They are sampling scintillator calorimeters which are read out with plastic fibers and phototubes.

\subsubsection{Central Calorimeter}

The Central Electromagnetic Calorimeter [52] detects electrons and photons and measures their energy. It consists of a lead-scintillator sampling system with tower segmentation. Each tower has an angle of $15^{\circ}$ in $r-\phi$ plane. The CEM total thickness of CEM is 18 radiation length $(32 \mathrm{~cm})$, to make sure that $99.7 \%$ of the electrons energy 
will be deposited. The energy resolution of CEM is

$$
\frac{\sigma_{E}}{E}=\frac{13.5 \%}{\sqrt{E_{T}}} \oplus 2 \%
$$

where $E_{T}$ is the transverse energy in $\mathrm{GeV}, \oplus$ symbol means that the constant term is added in quadrature to the resolution, and position resolution is typically $2 \mathrm{~mm}$ for 50 $\mathrm{GeV} / c$ electrons.

The Central Electromagnetic Showermax Chamber (CES) is used to identify electrons and photons using the position measurement to match with tracks, the transverse shower profile to separate photons from $\pi^{0} \mathrm{~s}$, and pulse height to help identify electromagnetic showers. The CES is located at approximately 6 radiation lengths deep at the expected shower maximum of particles in the EM calorimeter. The CES module is a multi-wire proportional chamber with 64 anode wires parallel to the beam axis.

The Central Preshower Detector (CPR) [53] is located between the front face of the EM calorimeter and the magnet coil. The CPR can be useful in the $\pi$-photon separation and electron identification. The CPR was replaced the slow gas chamber with a faster scintillator version which has a better segmentation during RunII in 2004. The new CPR is used to improve the jet energy resolution.

The Central Hadronic Calorimeter [54] is an iron-scintillator sampling calorimeter, covering in the range $|\eta|<0.9$, approximately $4.5 \lambda_{0}$ interaction length, and the energy resolution is

$$
\frac{\sigma_{E}}{E}=\frac{50.0 \%}{\sqrt{E_{T}}} \oplus 3 \% \text {. }
$$

The Wall Hadronic Calorimeter (WHA) is also an iron-scintillator sampling calorimeter, covering in the range $0.7<|\eta|<1$.3. The WHA is $4.5 \lambda_{0}$ interaction length, and the energy resolution is

$$
\frac{\sigma_{E}}{E}=\frac{75.0 \%}{\sqrt{E_{T}}} \oplus 4 \%
$$

\subsubsection{Plug Calorimeter}

The plug calorimeter covers $1.1<|\eta|<3.6$, corresponding to polar angles $3^{\circ}<\theta<$ $37^{\circ}$ as shown in Figure 2.11. Each plug wedge spans $15^{\circ}$ in azimuth, however from $1.1<|\eta|<2.11\left(37^{\circ}\right.$ to $\left.14^{\circ}\right)$ the segmentation in $\phi$ is doubled, and each tower spans only $7.5^{\circ}$. There is an electromagnetic section (PEM) with a shower position detector (PES), followed by a hadronic section (PHA).

The PEM [55] is lead-scintillator sampling calorimeter, with unit layers composed of $4.5 \mathrm{~mm}$ lead and $4 \mathrm{~mm}$ scintillator. There are 23 layers in depth for a total thickness of about $21 X_{0}$ radiation length at a normal incidence. The PEM has an energy resolution of

$$
\frac{\sigma_{E}}{E}=\frac{16 \%}{\sqrt{E_{T}}} \oplus 1 \%
$$


The PHA is an iron-scintillator sampling calorimeter, approximately $7 \lambda_{0}$ in depth, and has an energy resolution of

$$
\frac{\sigma_{E}}{E}=\frac{80 \%}{\sqrt{E_{T}}} \oplus 5 \%
$$

The PEM shower maximum detector is located about $6 \lambda_{0}$ deep within the PEM, and is constructed by two layers of scintillating strips. The strips are $5 \mathrm{~mm}$ wide, and roughly square in cross section. Position resolution of the PES is about $1 \mathrm{~mm}$. The summaries of design parameters for the calorimeter are shown in Table 2.4.

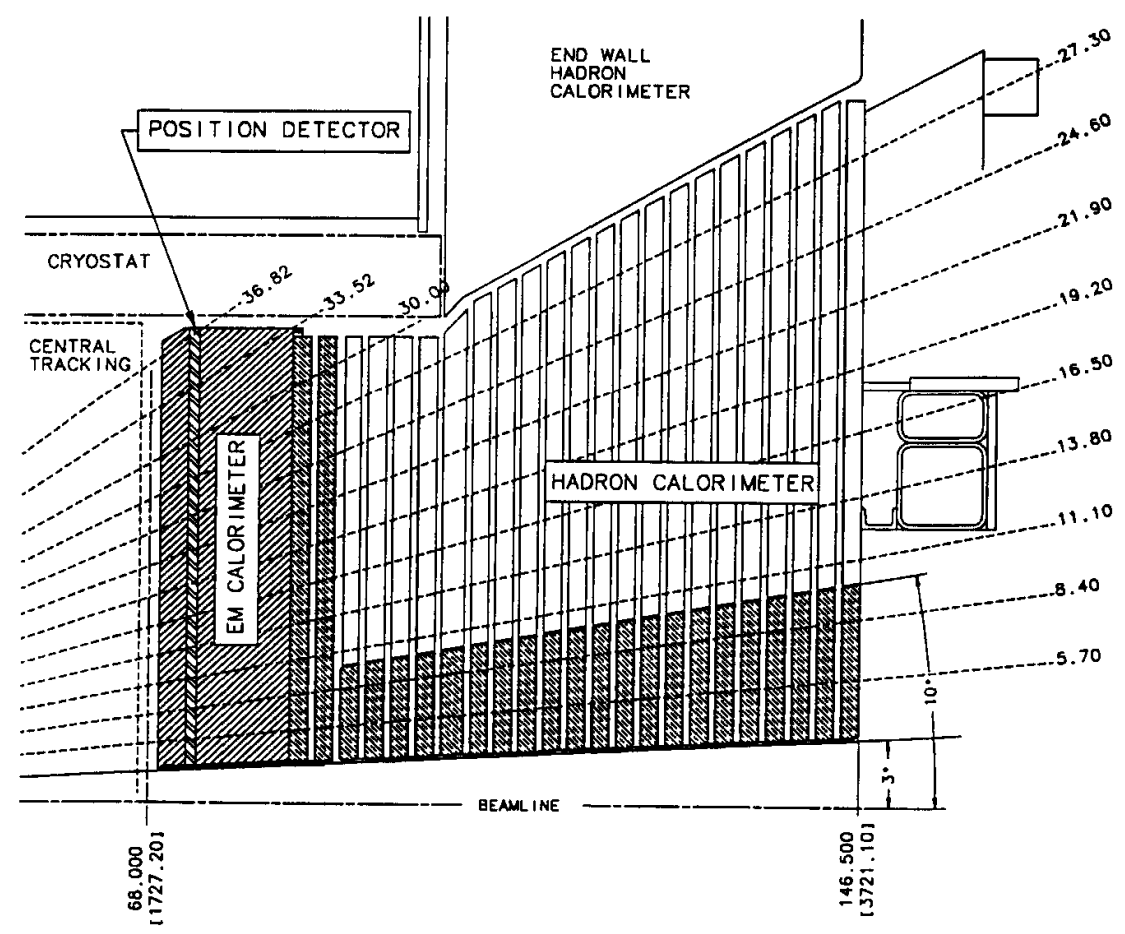

Figure 2.11: Cross section of the plug calorimeter (PEM and PHA).

\subsection{Muon Detectors}

Muons penetrate the tracking systems and the calorimeters leaving very little energy. The reason is that muons produce much less bremsstrahlung than electrons, and therefore do not produce electromagnetic showers, due to their larger mass. The CDF muon systems [44] use this property by placing detectors behind enough materials. Muons deposit minimum ionizing energy in the calorimeters matched with a track in the COT. 


\begin{tabular}{lcccc}
\hline \hline Calorimeter & Coverage & Energy Resolution $(\%)$ & Thickness & Absorber \\
\hline CEM & $|\eta|<1.1$ & $13.5 / \sqrt{E_{T}} \oplus 2$ & $18 X_{0}$ & $3.18 \mathrm{~mm} \mathrm{lead}$ \\
PEM & $1.1<|\eta|<3.6$ & $16.0 / \sqrt{E_{T}} \oplus 1$ & $21 X_{0}$ & $4.5 \mathrm{~mm}$ lead \\
CHA & $|\eta|<0.9$ & $50.0 / \sqrt{E_{T}} \oplus 3$ & $4.5 \lambda$ & $2.5 \mathrm{~cm}$ iron \\
WHA & $0.7<|\eta|<1.3$ & $75.0 / \sqrt{E_{T}} \oplus 4$ & $4.5 \lambda$ & $5.0 \mathrm{~cm}$ iron \\
PHA & $1.3<|\eta|<3.6$ & $80.0 / \sqrt{E_{T}} \oplus 5$ & $7.0 \lambda$ & $5.08 \mathrm{~cm}$ iron \\
\hline \hline
\end{tabular}

Table 2.4: Design parameters of the calorimeter.

The momentum of these muons is measured by their bending in the solenoidal field using the COT. The central muon system is capable of detecting with transverse momentum $p_{T} \geq 1.4 \mathrm{GeV}$, through their interaction with the gas and subsequent drift on the produced electrons toward the anode wires. The muon detectors consist of four separate subsystems: the central muon chambers (CMU), the central upgrade (CMP), the central muon extension (CMX), and the barrel muon detector (BMU). Table 2.5 shows design parameters of the muon detector. Figure 2.12 shows the effective muon detector coverage in $\eta-\phi$ plane.

The CMU detector locates at $35 \mathrm{~m}$ from the interaction point and outside of the central hadron calorimeter. It covers the region of $|\eta| \leq 0.6$. It is divided into 24 east and 24 west $15^{\circ}$ wedges. Each wedge contains three muon chambers and each muon chamber consists of four layers of four rectangular drift cells staggered in order to eliminate hit position ambiguities. A stainless steel sense wire a diameter of $50 \mu \mathrm{m}$ is located in the center of each cell. A muon object is created by forming a "stub" from hits in the muon chambers matching it to an extrapolated COT tracks.

The CMP consists of a second set of muon chambers behind additional $60 \mathrm{~cm}$ of steel in the region $55^{\circ} \leq \phi \leq 90^{\circ}$. The chambers are fixed length in $z$ and form box around the central detector. The coverage in terms of pseudorapidity varies with azimuth as shown in Figure 2.12.

The central extension consist of conical section of drift tubes (CMX) in polar angle from $42^{\circ}$ to $55^{\circ}(0.6 \leq|\eta| \leq 1.0)$. The top two wedges (Wedge 5 and 6$)$ of the west CMX is called "Keystone". There are no top 2 wedge on the east CMX due to cryogenic utilities servicing the solenoid. The bottom 6 wedges (Wedge 15-20) are called "Miniskirt". The design parameters of the muon detector are shown in Table 2.5.

\subsection{Luminosity Monitor}

The beam luminosity has been measured using the process of inelastic $p \bar{p}$ scattering. The cross section $\sigma_{\text {in }}$ is $\sim 60 \mathrm{mb}$. The rate of inelastic $p \bar{p}$ interaction is given by

$$
\mu f_{\mathrm{BC}}=\sigma_{\mathrm{in}} L
$$




\begin{tabular}{lccc}
\hline \hline Muon detector & CMU & CMP & CMX \\
\hline Coverage & $|\eta|<0.6$ & $|\eta|<0.6$ & $0.6<|\eta|<1.0$ \\
Drift tube length $[\mathrm{cm}]$ & 226 & 640 & 180 \\
Max drift time $[\mu \mathrm{s}]$ & 0.8 & 1.4 & 1.4 \\
Total drift tubes & 2304 & 1076 & 2208 \\
Pion interation length $(\lambda)$ & 5.5 & 7.8 & 6.2 \\
Minimum detectable muon $p_{T}(\mathrm{GeV} / c)$ & 1.4 & 2.2 & 1.4 \\
\hline \hline
\end{tabular}

Table 2.5: Design parameters of the moun detector.

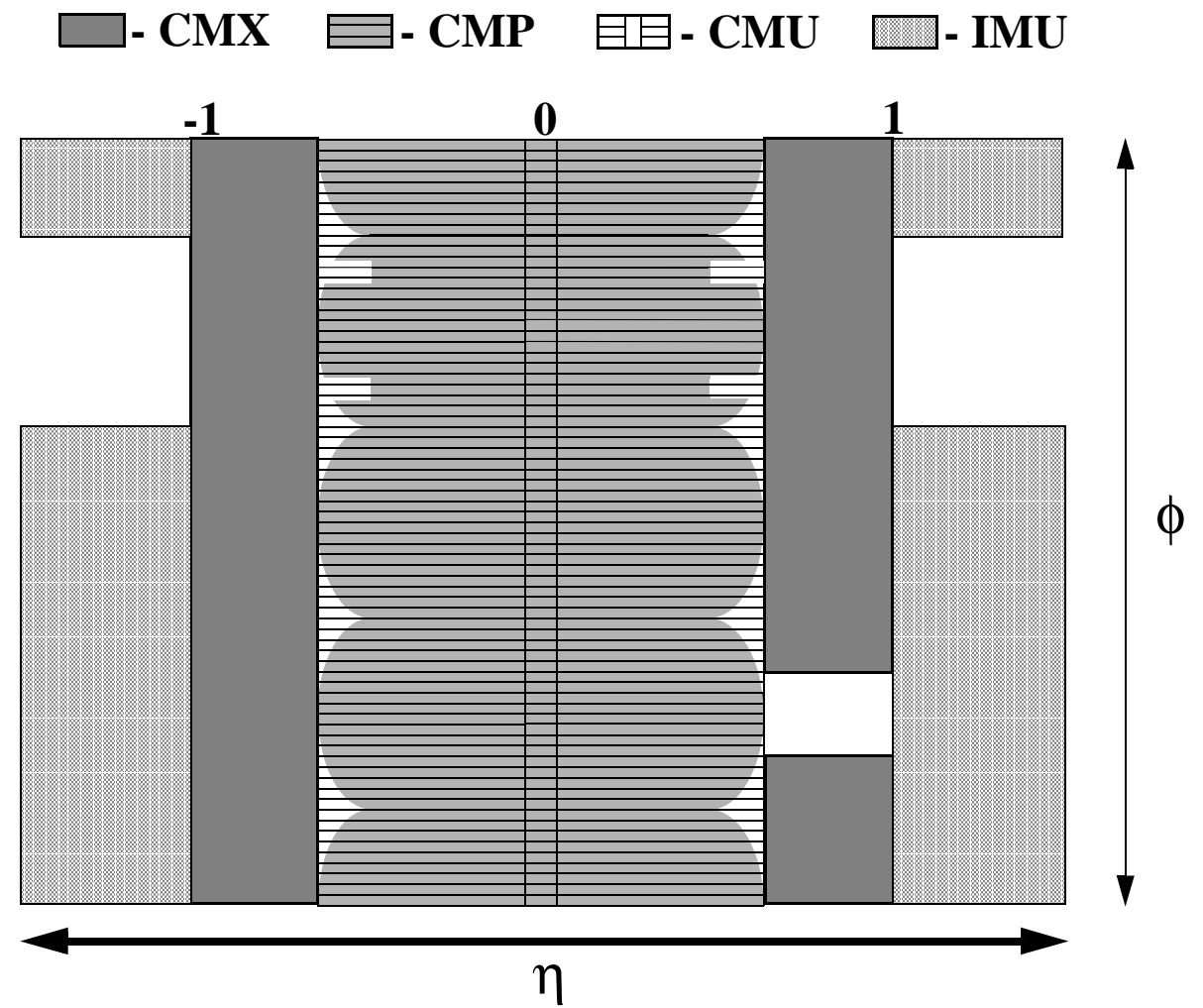

Figure 2.12: Muon detector coverage in $\eta-\phi$ plane.

where $L$ is the instantaneous luminosity, $f_{\mathrm{BC}}$ is the rate of bunch crossing in the Tevatron and $\mu$ is the average number of $p \bar{p}$ interaction per bunch crossing. In CDF Run II, Cherenkov luminosity counters (CLC) $[56,57]$ is used to measure the luminosity by counting the number of $p \bar{p}$ interaction $\mu$ accurately.

The detector consists of two modules. They are located in the " 3 degree holes" inside the end-plug calorimeter in the forward and backward region and which cover $3.7<|\eta|<4.7$ range. Each CLC detector module consists of 48 thin, long, conical, gas- 


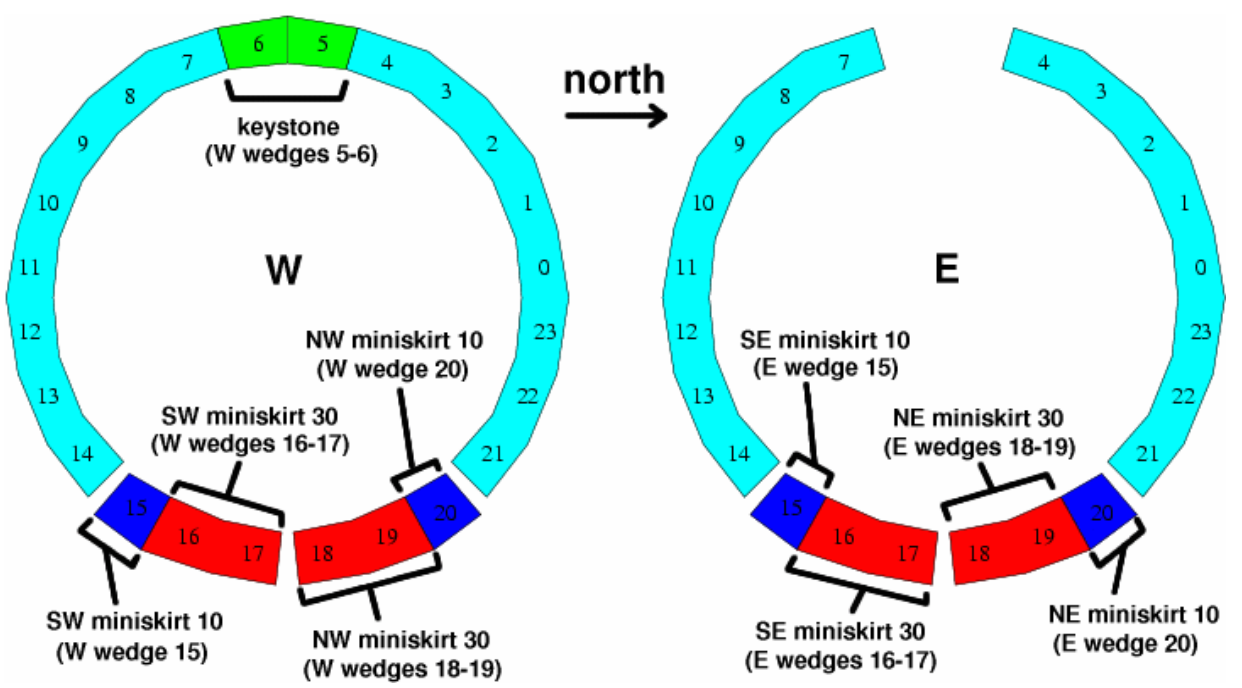

Figure 2.13: CMX detector in $r-\phi$ plane.

filled Cherenkov counters. The counters are arranged around the beam pipe in three concentric layers, with 16 counters each, and pointing to the center of the interaction region. They are built with reflective aluminized mylar sheets of $0.1 \mathrm{~mm}$ thick and have a conical shape. The cones in two outer layers are about $180 \mathrm{~cm}$ long and the inner layer counters have the length of $110 \mathrm{~cm}$. The Cherenkov light is detected with fast, $2.5 \mathrm{~cm}$ diameter, photomultiplier tubes. The tubes have a concave-convex, $1 \mathrm{~mm}$ thick, quartz window for efficient collection of the ultra-violet part of Cherenkov spectra and operate at a gain of $2 \times 10^{5}$. The counters are mounted inside a thin pressure vessel made of aluminum and filled with isobutane. The systematic uncertainty of the luminosity measurement is dominantly coming from the uncertainty of the inelastic $p \bar{p}$ cross section $(\sim 3 \%)$, the CLC acceptance $(\sim 2 \%)$, and the non-lineality of the CLC acceptance due to CLC occupancy saturates as growing luminosity due to the finite number of counters $(<2 \%)$.

\subsection{Trigger Systems}

The trigger plays an important role on hadron collider experiment because the collision rate is much higher than the rate as which data can be stored on tape. The crossing rate of the Tevatron under 36 on 36 bunch operation is $7.6 \mathrm{MHz}$, corresponding to $396 \mathrm{~ns}$ collision separation. The role of the trigger is to effectively extract the most interesting physics events from the large number of minimumally biased events. For Run II, CDF employs a three-level trigger system. The levels are denoted simply as "L1", "L2" and "L3", with each subsequent level making more complicated decisions and requiring successively longer processing times. Figure 2.14 shows a schematic illustration of the CDF trigger system. 
RUN II TRIGGER SYSTEM

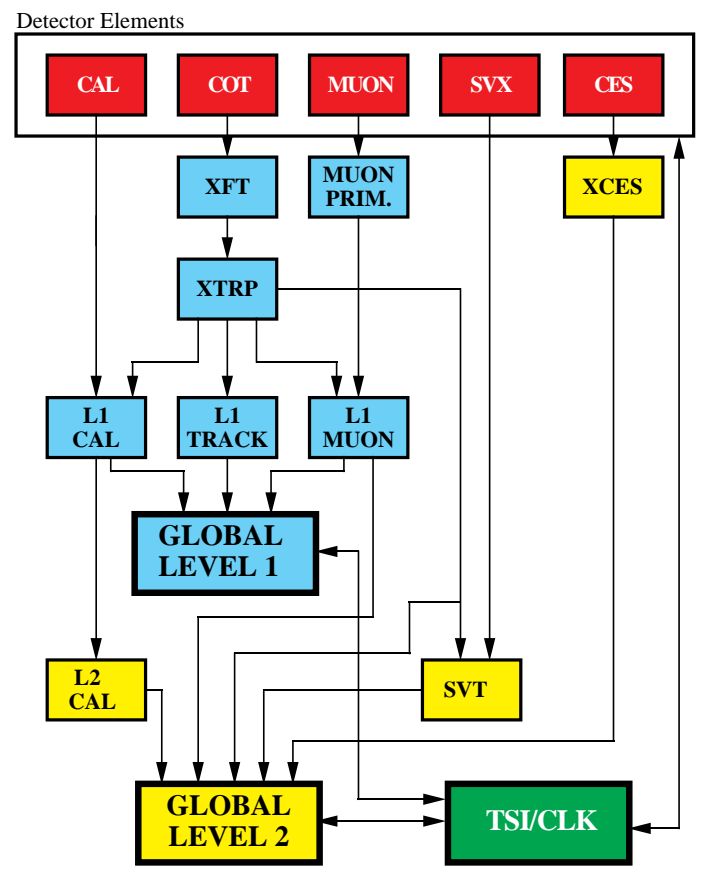

Figure 2.14: Book diagram of the trigger pass for Level 1 and Level 2.

\subsubsection{Level-1}

The first level of trigger selection Level-1 (L1) uses custom designed hardware to find physics objects based on a subset of the detector information and then makes a decision based on simple counting of these objects. The input to the L1 hardware comes from the calorimeters, tracking chambers and muon detectors. The decision to retain an event for further processing is based on the number and energies of the electron, jet and muon candidates as well as the missing energy in the event, or on the kinematic properties of few of these objects. The L1 hardware consists of three parallel synchronous processing streams which feed inputs of the single Global Level-1 decision unit. One stream finds calorimeter objects, another finds muons and the third finds tracks in the central region. The L1 trigger can be formed using these streams singularly as well as AND or OR combinations of them. All elements of the L1 trigger are synchronized to the same 132 ns clock, with a decision made every $132 \mathrm{~ns}$ by Global L1. In the period of the data taking considered in this analysis the accelerator was the two intermediate clock cycles automatically rejected. The maximum L1 accept rate is $20 \mathrm{kHz}$, while the typical one is $12 \mathrm{kHz}$. 


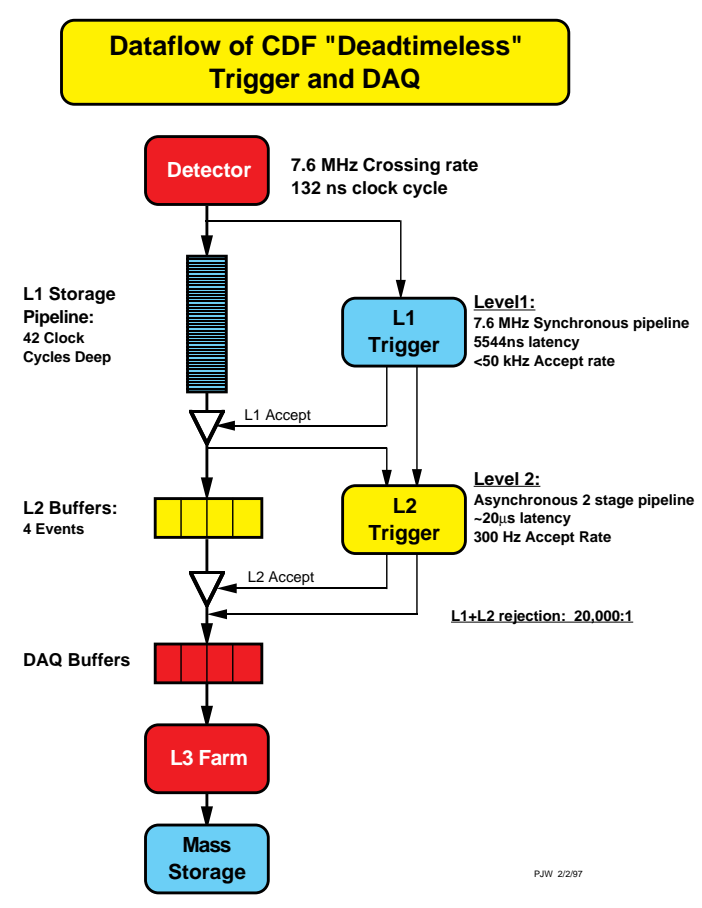

Figure 2.15: Schamatic diagram of the trigger and DAQ.

\subsubsection{Level-2}

Events accepted by L1 are processed by the second level of trigger Level-2 (L2), which is composed of several asynchronous subsystems. These provide input data to programmable L2 processors on the Global L2 crate. The L2 processors determine if any of the L2 trigger are satisfied. Processing for L2 trigger decision starts after the event written into one of the four L2 buffers by a L1 accept. When L2 is analyzing the event in one of the buffers, that buffer cannot be used additional L1 accept. If all the four are full, the deadtime of the data acquisition is increased. It follows that the time required for a L2 decision needs to be less than about $80 \%$ of the average time between L1 accepts in order to keep the deadtime as low as possible. For this purpose L2 has been pipelined into two stages each taking approximately $10 \mu \mathrm{s}$, which is sufficient to keep the deadtime at a minimum, even if L1 had an accept-rate of 50kHz. The L2 buffers perform a limited event reconstruction using essentially all the information used in L1, but with higher precision. In addition, at L2, data from the central shower-max detector and the SVX are available, which improve respectively the identification of electrons and photons and the reconstruction of the secondary vertices. Furthermore, a jet reconstruction algorithm is provided by the L2 cluster finder. After all of the data are stored in the processors, the event is examined to check if the criteria of any of the L2 triggers have been satisfied. This operation can be performed while the new events are being loaded into memory, thus not affecting the dead time. The typical L2 accept rate, as of this writing, is between 100 and $300 \mathrm{~Hz}$, depending on the initial luminosity. 


\subsubsection{Level-3}

The Level-3 (L3) trigger subsystem is composed of two main components, the Event Builder (EVB) and the Level-3 Farm. Level-1 and Level-2 systems need to make their decisions at very high rate which makes it impossible to fully reconstruct each event. While Level-1 and Level-2 algorithms use small predefined pieces of event data to make their decision, the event pieces are stored in the buffers of the 140 Front End crates which constitute the EVB. After a L2 decision is made, the Event Builder assembles all event fragments from the Front End crates into one data block.

The 16 subfarms which compose the L3 Farm receive event fragments from the EVB and build complete events into the appropriate data structure for analysis. Since it takes about one second for one computer unit to make a trigger decision on one event, it takes a large farm of 250 Dual Pentiun Linux personal 5computers (called "processors") to ensure the required input rate. Each subfarm contains between 14 and 18 processor nodes and one "converter" node, which acts as "farm input" distributing the data flow coming from the EVB.

The events are then passed to a trigger algorithm (a different one for each processor) that categorizes the event and makes the decision as to whether or not to permanently store it. The selected event are passed to the Data Logger subsystem. During the building processing, the event integrity is checked. The L3 algorithms take advantage of the full detector information and improved resolution unavailable to lower trigger levels. This includes full three-dimensional track reconstruction and tight matching of tracks to calorimeter and muon-system information. Results from the lower level are used or drive the algorithms, which are based on the off-line analysis packages. This is a modular and separated filter modules for specific triggers. L3 accept events with a rate of approximately $75 \mathrm{~Hz}$. 


\section{Chapter 3}

\section{Dataset and Event Selection}

Physics objective in this thesis is to search for the neutral Higgs boson associated with a vector boson using like-sign dilepton events $(e e, e \mu$, and $\mu \mu)$, and a such event occurs in the following process,

$$
q q^{\prime} \rightarrow V h \rightarrow V W^{*} W^{*} \rightarrow \ell^{ \pm} \ell^{ \pm}+X, \quad V=W, Z .
$$

The interesting events are collected by using trigger systems as described in the previous chapter.After that, the events are applied in a series of selections. This chapter describes the triggers and the selections.

\subsection{Dataset and Triggers}

The event of the Higgs boson, eq. (3.1), has typically one high- $p_{T}$ lepton. Therefore, it is desirable to select the data which is collected by an inclusive high- $p_{T}$ lepton (electron and muon) trigger.

The inclusive high- $p_{T}$ electron trigger requires at least one electron satisfied the series of electron selections and some large $E_{T}$ requirement. Concretely, the trigger selects events having an object which deposit some energy to electromagnetic calorimeter $\left(E_{T}>18 \mathrm{GeV}\right)$, the ratio of the energy deposition of EM calorimeter to the hadron calorimeter (HAD/EM) is less than 0.124, some requirement of the lateral shower profile $\left(L_{\mathrm{shr}}\right)$, and the position matching on $z$ direction between CES and extrapolated track $\left(\Delta z_{\mathrm{CES}}<8 \mathrm{~cm}\right)$. The criteria is applied to events step by step, i.e. Level 1, Level 2 , and Level 3, to reduce the data taking rate due to the capability limit for the trigger system. The trigger path is named ELECTRON_CENTRAL_18_v*, where asterisk symbol means the version number because the trigger criteria are changed in run by run due to the performance and condition of CDF detector and Tevatron accelerator.

The inclusive high- $p_{T}$ muon trigger requires at least one muon satisfied the series of muon selections and some large $p_{T}$ requirement. The muon trigger is mainly classified into a CMUP muon trigger and a CMX muon trigger. The CMUP muon means a track object points to both CMU and CMP detector, while CMX muon points to 
CMX detector. The CMUP muon trigger is named MUON_CMUP18_*, which requires CMUP muon with XFT track $p_{T}>18 \mathrm{GeV} / c$ and the position matching in $x$ direction between the position on muon detectors, both CMU and CMP, and track. And the CMX muon trigger (named MUON_CMX18_*) requires CMX muon with $p_{T}>18 \mathrm{GeV} / c$ and the position matching same as the case of CMUP muon trigger, but in this case, CMX detector.

The collected data are achieved during from February 4th in 2002 to September 30 th in 2011 corresponding to an integrated luminosity $9.4 \mathrm{fb}^{-1}$ data after run filtering for good detector condition and desirable, so-called "good run filtering". In CDF RunII, the data is categorized into some dataset with respect to data taking span, e.g. Od, or Period 0 , to calibrate the data take into account the varying taking data condition due to the long range operation. The categorized dataset and the run range are shown in Table 3.1. The main features of the triggers are shown in Tables 3.2, 3.3, and 3.4. The method how to estimate the trigger efficiency is explained in Section 4.3.3 and the efficiency in each period is shown in A.1.

\subsection{Event Selection}

CDF-II has well-defined algorithm to identify electrons or muons by using track reconstruction, energy clustering, and characteristic reactions to detectors. These efficiencies are basically estimated by using $Z \rightarrow \ell \ell$ control samples. The detail how to estimate the efficiencies is explained in Section 4.3.4 and 4.3.5. The selection efficiency in each dataset is shown in A.2 and A.3.

\subsubsection{Pre-Event Selection}

The pre-event selections are first applied. The selections consists of two parts. One is to ensure well-defined measurement of collisions with the detector. It requires the vertex with the highest $p_{T}$-sum of associated tracks, so-called "primary vertex", within the region of the $z$-axis $\left|z_{\mathrm{pv}}\right|<60 \mathrm{~cm}$.

The other is a veto of the cosmic rays. The cosmic rays can contaminate physics events, coming from collision, by mimicking muons or electrons. The cosmic rays are coming from outside of the detector, while the muon with collisions are coming from center of detector. Furthermore, the cosmic rays cross the detector at any time with respect to the beam crossing. The veto is possible by looking at the direction of the trajectory and the crossing timing.

\subsubsection{Lepton Identification}

In this thesis, central electrons (CEM), central muons (CMUP, CMX, CMU, CMP), forward muons (BMU), and stubless muons (CMIO-CES for central region and CMIOPES for forward region) are used to search for the Higgs boson. The selections are 
categorized into 3 parts, "geometrical and kinematics cuts", "track quality cuts", and "identification cuts (ID cuts)".

\section{Geometrical and kinematical cuts}

- Electron Fiducial:

This variable ensures that one electron is reconstructed in the region of the detector instrumented well. A position of the electron in the CEM is determined using a value determined by the CES or an extrapolated track. It must satisfy the following requirements:

- The electron must lie within $21 \mathrm{~cm}$ of the tower center in the $r-\phi$ view in order for the shower to be fully contained in the active region $\left|z_{\mathrm{CES}}\right|<21 \mathrm{~cm}$.

- The electron should not be in the regions of $\left|z_{\mathrm{CES}}\right|<9 \mathrm{~cm}$, where the two halves of the central calorimeter meet, and $\left|z_{\mathrm{CES}}\right|>230 \mathrm{~cm}$, which corresponds to outer half of the last CEM tower. This region is prone to leak the energy into the hadronic part of the calorimeter.

- The electron should not be in the region close to the point of penetration of the cryogenic connections to the solenoidal magnet, where is uninstrumented. This corresponds to $0.77<\eta<1.0,75<\phi<90$ degree, and $\left|z_{\mathrm{CES}}\right|<193 \mathrm{~cm}$.

- Muon Fiducial:

Muons are identified using the information of hits in the muon chambers with a reconstructed track and energy in the calorimeter. Muons pass through the muon chambers, then the muon tracking is formed using the hit information and fitting algorithm (Muon (stub) reconstruction). The muon stub has at least three hits associated to it. This stub requirement is for CMUP, CMX, CMU, CMP and BMU muons.

- The fiducial distance of the tracks extrapolated to muon chambers in the $r-\phi$ plane and $z$-direction.

- COT exit radius $\rho$ :

To ensure that CMX muon pass through all eight COT superlayers, CMX muons require COT exit radius $\rho$ of the track. $\rho$ is defined as,

$$
\rho=\frac{\eta}{|\eta|} \cdot \frac{z_{\mathrm{COT}}-z_{0}}{\tan (\pi / 2-\theta)}
$$

where $z_{\mathrm{COT}}$ is used for the length of the $\mathrm{COT}(155 \mathrm{~cm})$.

- High transverse energy $\left(E_{T}\right)$ :

The transverse electromagnetic energy deposited by electron is calculated as the electromagnetic cluster energy multiplied by $\sin \theta$, where $\theta$ is the polar angle provided by the best COT track pointing to the EM cluster. 
- High transverse momentum $\left(p_{T}\right)$ :

The transverse momentum of the COT track is measured by using the track curvature in the COT.

\section{Track quality cuts}

- COT hits requirement:

It ensures that a track associated with one electron or muon is a well-reconstructed track. It requires that the track has been reconstructed in the COT in 3 axial and 3 stereo superlayers with at least 7 hits in each.

- The relative position to the primary vertex in the $z$ plane $\left(z_{0}-z_{\mathrm{pv}}\right)$ :

Separation between $z$ coordinate of the closest approach point with respect to run average beam line $\left(z_{0}\right)$ and primary vertex $z$ position $\left(z_{\mathrm{pv}}\right)$.

- Silicon hits requirement:

A track is satisfied with the hits at some SVX layers ( $>3)$. This requirement plays a roll of rejecting the residual photon conversion events, which are considerable background in the LS dilepton events.

- Impact parameter $\left(d_{0}\right)$ :

This variable is recalculated to take the $x$ coordinate of the primary vertex. The cuts is the most powerful for rejecting backgrounds of cosmic rays.

\section{Isolation cut}

- Isolation ( $\left.\mathrm{ISO}_{0.4}^{\mathrm{cal}}\right)$ :

The leptons are required to be isolated in terms of the calorimeter cone-isolation with a cone size of $\Delta R=0.4\left(\Delta R=\sqrt{\Delta \eta^{2}+\Delta \phi^{2}}\right)$. The calorimeter isolation is defined for track objects. It is

$$
\mathrm{ISO}_{0.4}^{\text {cal }}=\sum_{\Delta R<0.4} E_{T}^{(i)}-\left(E_{T}^{(\mathrm{seed})}+E_{T}^{(\eta+1)}+E_{T}^{(\eta-1)}\right),
$$

where $E_{T}^{(i)}$ is the tower $E_{T}$ summed over the electromagnetic and hadronic calorimeter, $E_{T}^{(\text {seed })}$ is the $E_{T}$ of the tower that the track is pointing, and $E_{T}^{(\eta \pm 1)}$ is the same quantities for the towers in the same wedge but with the $\eta$ index off by the 1 with respect to the seed tower.

\section{Conversion removal}

A photon traveling through material converts into an electron-positron pair. However the electron is a real electron, it does not a prompt electron, that is, not coming from a hard scattering event. The electron is vetoed if oppositely charged electron is found satisfied with the condition of

$$
|\Delta \cot \theta|<0.04 \text {, and }\left|\delta_{x y}\right|<0.2,
$$


$\cot \theta$ is the difference of the cotangents of the polar angles between these tracks, $\delta_{x y}$ is the separation between the tracks in the $r-\phi$ plane.

\section{Likelihood-Based Lepton Identification}

We know that fake-lepton backgrounds can be reduced by LS requirement, but it is still remains at a considerable level [58]. To reduce fake-lepton backgrounds significantly, we employ likelihood-based lepton identification (LLID), which combines information from several variables and outputs a single value. As control samples for real and fake leptons, we choose leptons from $Z$ candidate in high- $p_{T}$ triggered data and fake leptons in jet samples. Both are applied geometrical and kinematical cuts, track quality cuts, and isolation cuts. Then, probability distribution functions (PDFs) are constructed in each variable to be used as a lepton identification in each control sample. PDFs are created by means of histograms, not functions. Therefore, PDFs satisfy

$$
\sum_{i=0}^{\text {nbins }}(\mathrm{PDF})_{i} \times d_{i}=1,
$$

where $d_{i}$ is the bin width in $i$ bin. We define a likelihood function that a lepton candidate is indeed a real lepton to be

$$
\mathcal{S}=\prod_{i=1}^{n} S_{i},
$$

where $S_{i}$ is the PDF of real leptons for the $i$-th ID variable, with ignoring any correlations between ID variables. The likelihood that the lepton candidate is a fake lepton is to be given by

$$
\mathcal{B}=\prod_{i=1}^{n} B_{i}
$$

Then, we construct the likelihood estimator as

$$
\mathcal{L}=\frac{\mathcal{S}}{\mathcal{S}+\mathcal{B}}
$$

so as to be $0 \leq \mathcal{L} \leq 1$. We set the likelihood cut value on 0.70 for electrons, and 0.20 for CMUP and CMX muons, and 0.90 for other muon types. In the following, variables used as PDFs are shown. The PDFs are shown in Figures 3.1.

\section{Electron Identification Variables}

- Ratio of hadronic and electromagnetic energy (HAD/EM):

This quantity is the ratio of 2-tower hadronic energy deposition in the CHA/WHA to the 2-tower electromagnetic energy deposition in the CEM. The ratio should be small, that is, energy deposition in electromagnetic calorimeter is much higher than energy deposition in hadronic calorimeter. 
- EM shower shape $\left(L_{\text {shr }}\right)$ :

The purpose of this quantity is to provide some discrimination of electrons and photons from hadronic showers faking these particles in the CEM. This is done by comparing the observed energy in CEM towers adjacent to the seed tower to expected electromagnetic shower taken with test beam data:

$$
L_{\mathrm{shr}}=0.14 \sum_{i} \frac{E_{i}^{(\mathrm{adj})}-E_{i}^{(\exp )}}{\sqrt{(0.14 \sqrt{E})^{2}+\left(\Delta E_{i}^{\exp }\right)^{2}}},
$$

where $E_{i}^{\text {adj }}$ is the measured energy in the tower adjacent to the seed tower, $E_{i}^{\text {exp }}$ is the expected energy in the adjacent tower from test beam data, $\Delta E_{i}^{\exp }$ is the error on the energy estimation.

- Ratio of the cluster energy to the momentum $(E / p)$ :

This is defined by the ratio of the cluster energy to the beam-constrained COTtrack momentum. To clarify, $E / p$ in the electron selection is not $E_{T} / p_{T}$. If a object pointing calorimeter cluster is electron, its momentum measured by COT track matches to the energy in the calorimeter cluster, i.e. $E / p \sim 1$.

- The pulse height shape in CES $\left(\chi_{\text {strip }}^{2}\right)$ :

The pulse height shape in the strip view $(r-z)$ of the CES detector is compared to the same profile extracted from the test-beam data of electrons using $\chi^{2}$ test.

- Track matching to CES cluster $\left(Q \times \Delta x_{\mathrm{CES}}\right)$ :

$\Delta x_{\mathrm{CES}}$ is the distance, in units of $\mathrm{cm}$, in the $r-\phi$ plane between the track extrapolated to the radius of the CES and the actual cluster position measured by the CES. If the sign of charge and $\Delta x$ is opposite, the track traverses a larger part of the calorimeter in adjacent towers, which results in more radiation and a less precise position.

- $\Delta z_{\mathrm{CES}}$ :

This variable is the distance, in units of $\mathrm{cm}$, in the $r-z$ plane between the track extrapolated to the CES radius and the actual cluster position measured by the CES.

- $E_{\mathrm{CES}} / p^{*}$ :

This is the ratio of the wire cluster pulse height measured in the CES, corrected by chamber wraps, to $p^{*}=10(p / 10)^{\alpha}$, where $p$ is the track momentum in $\mathrm{GeV} / c$, and $\alpha$ is

$$
\alpha=0.85+0.15 \exp \left(-\frac{p}{15}\right)-\frac{p}{1000},
$$

as defined in [59]. The value for a real electron is expected to be around 1. The CES responses are sensitive to the shower development which is expected to be different between electrons and interactive pions. 
- $\mathrm{CPR}$ or CP2:

The response of the CPR or CP2 detector corresponding to an EM-calorimeter cluster in units of the number of minimum-ionizing-particles (MIPs). The peak position corresponding to one MIP is identified using muons. The CPR/CP2 responses are also sensitive to the shower-development as well as the CES responses. For electrons, the energy deposition in CPR tends to be greater than hadronic fake leptons.

\section{Muon Identification Variables}

- calorimeter energy deposition (EM and HAD):

Muons deposit small energy in the calorimeters due to minimum ionizations. Therefore, it is useful variable to distinguish real muons from fake muons coming from punch-through hadrons.

- Track-stub matching in $r-\phi$ plane $(r \times \Delta \phi)$ :

The track is required to match the muon stub in $r-\phi$ plane.

\subsubsection{Jet Reconstruction}

Quarks and gluons are observed as "jet" objects due to its fragmentation and radiation effects, as a result of collimated shower of particles. The energy of a jet is calculated from the energy deposited in the calorimeter towers using a cone clustering algorithm with a fixed cone size in which the center of the jet is defined as $\left(\eta^{\text {jet }}, \phi^{j e t}\right)$ and the size of the jet cone as $R=\sqrt{\left(\eta^{\text {tower }}-\eta^{\text {jet }}\right)^{2}+\left(\phi^{\text {tower }}-\phi^{\text {jet }}\right)^{2}}=0.4$. The algorithm of a jet clustering groups calorimeter towers with $E_{T i}<1 \mathrm{GeV}$. The algorithm is performed by first defining "Seed towers" has largest $E_{T i}$. The seed tower is used to build "clusters" with the size of $R=0.4$. The Transverse energy and position of the cluster is calculated by

$$
E_{T}^{j e t}=\sum_{i=0}^{N} E_{T i}, \phi^{j e t}=\sum_{i=0}^{N} \frac{E_{T i} \phi_{i}}{E_{T}^{j e t}}, \eta^{j e t}=\sum_{i=0}^{N} \frac{E_{T i} \eta_{i}}{E_{T}^{j e t}}
$$

where $N$ is the number of towers inside the radius $R$ with $E_{T i}>1 \mathrm{GeV}$. This procedure is repeated until the cluster centroid is stable. Overlapping jets are merged if their overlap is greater than $50 \%$. If the overlap is smaller than $50 \%$, each tower in the overlap region is assigned to the nearest jet. The measured jets are corrected to particle jet level or parent parton level by taking into account for the detector effects, radiation, and fragmentation effects. The corrected transverse momentum of the jet is expressed as follows,

$$
\begin{aligned}
p_{T}^{\text {parton }} & =\left(p_{T}^{\text {jet }} \times C_{\eta}-C_{\mathrm{MI}}\right) \times C_{\mathrm{Abs}}-C_{\mathrm{UE}}+C_{\mathrm{OOC}} \\
& =p_{T}^{\text {particle }}-C_{\mathrm{UE}}+C_{\mathrm{OOC}},
\end{aligned}
$$




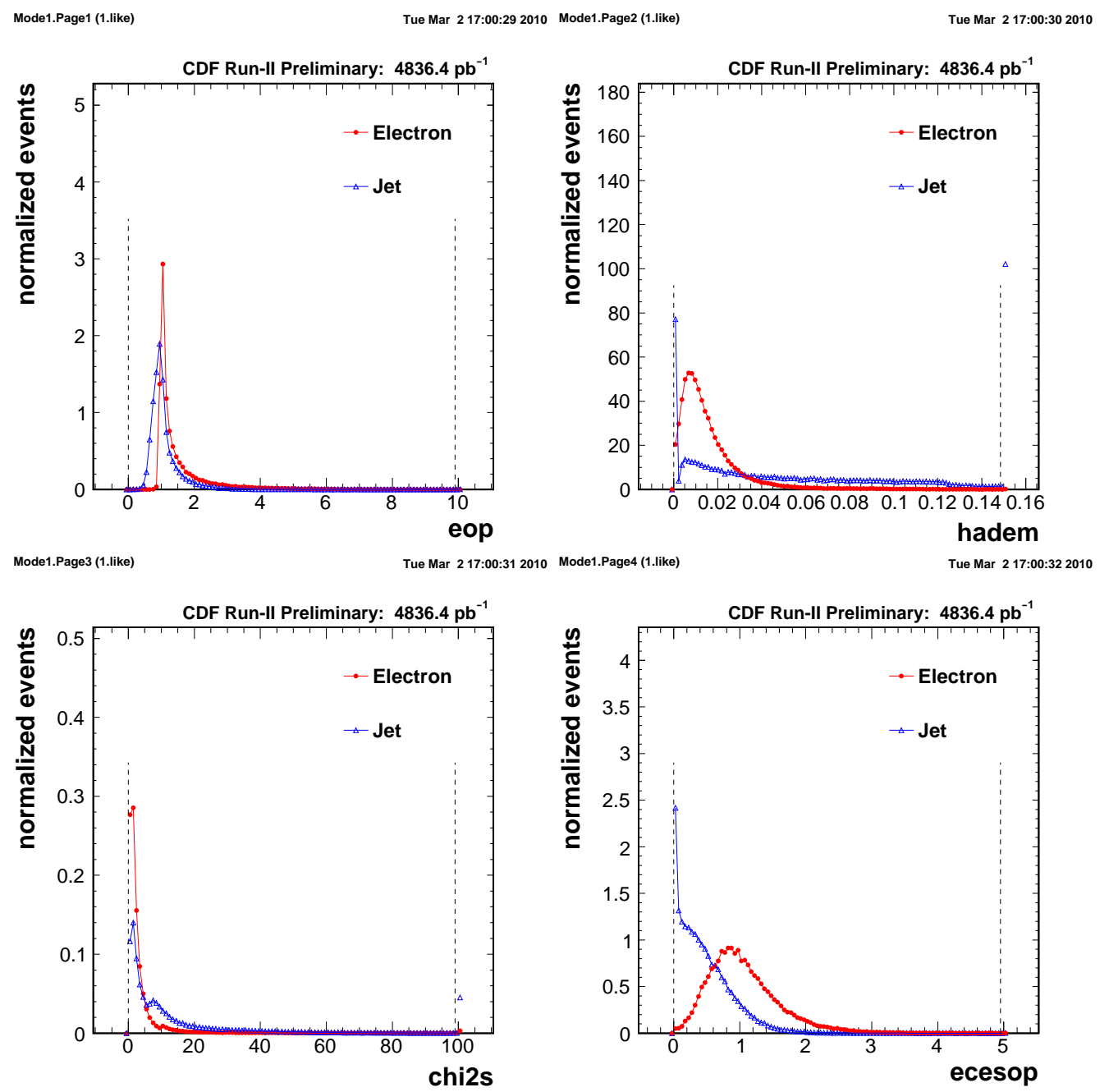

Figure 3.1: Normalized distributions of the identification variables for electrons from $Z$ decays compared to those for electron candidates in jet samples (top left: $E / p$, top right: $\mathrm{HAD} / \mathrm{EM}$, bottom left: $\chi_{\text {strip }}^{2}$, bottom right: $\left.E_{\mathrm{CES}} / p^{*}\right)$.

where $p_{T}^{\text {parton }}$ is the transverse momentum of the parent parton, which is taken into account for all effects, $p_{T}^{\text {jet }}$ is the transverse momentum measured in the calorimeter, $p_{T}^{\text {particle }}$ is the transverse momentum of the particle jet, which is corrected for detector effects, and

- $C_{\eta}$ is " $\eta$-dependent" correction. The correction takes into account variations in calorimeter response and gain as a function of jet $\eta$

- $C_{\mathrm{MI}}$ is "Multiple Interaction" correction, which is the energy coming from multiple $p \bar{p}$ interaction in the same bunch crossing to subtract from the jet

- $C_{\mathrm{Abs}}$ is "Absolute correction", is the correction of the calorimeter response to the momentum of the particle jet 


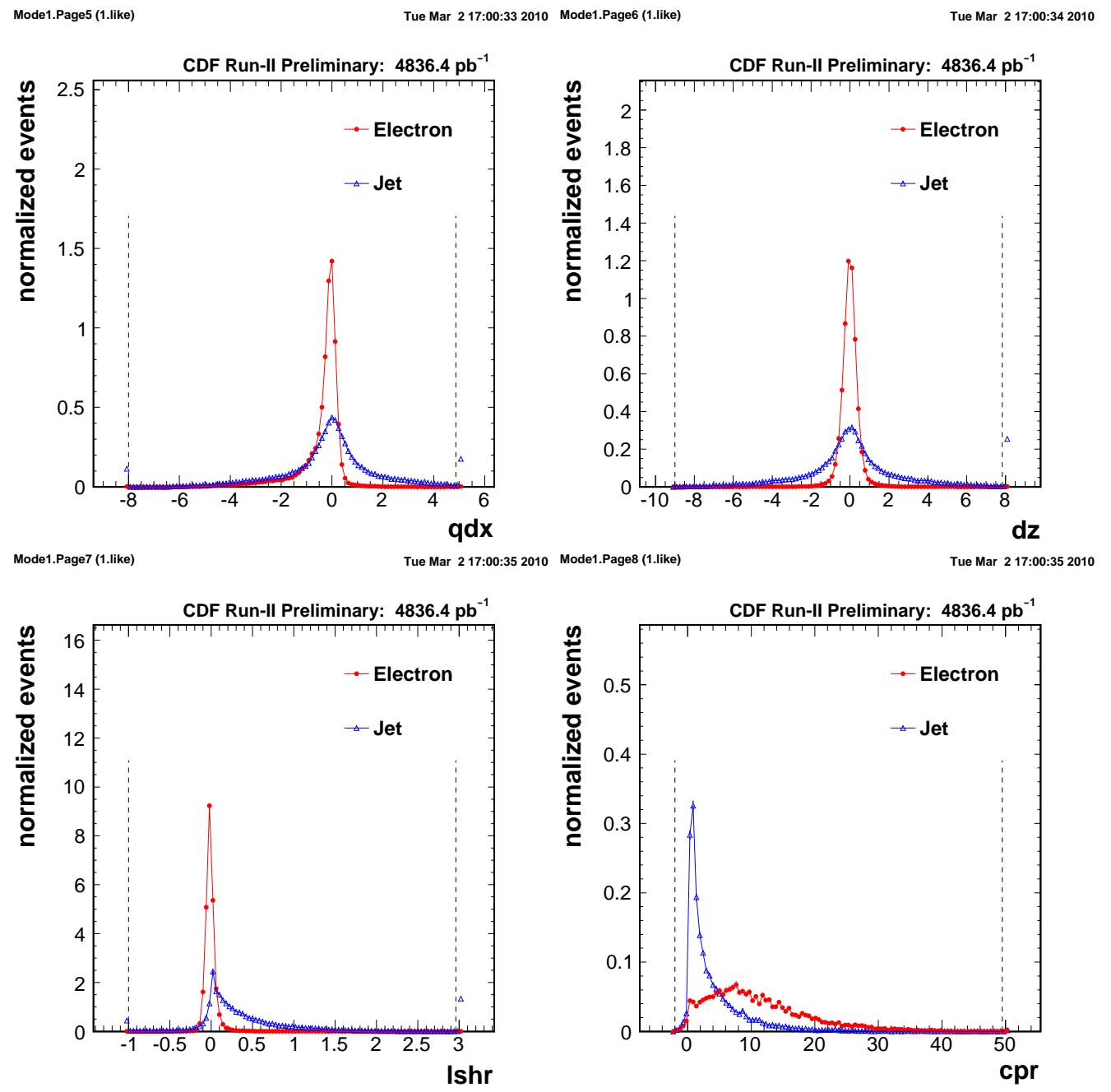

Figure 3.2: Normalized distributions of the identification variables for electrons from $Z$ decays compared to those for electron candidates in jet samples (top left: $Q \times \Delta x_{\mathrm{CES}}$, top right: $\Delta z_{\mathrm{CES}}$, bottom left: $L_{\mathrm{shr}}$, bottom right: CPR).

- $C_{\mathrm{UE}}$ is "Underlying Event correction", to remove energy coming from underlying event such as initial state radiation and beam-beam remnant

- $C_{\mathrm{OOC}}$ is "Out-of-Cone correction" is correction of parton radiation and hadronization effects due to the finite size of the jet cone algorithm

The corrections are performed by using the generic jet samples and MC samples generated by several generators (PYTHIA and HERWIG), and systematic uncertainties coming from these collections are also estimated. The contribution mainly comes from the absolute jet energy collection due to difference between data and MC for calorimeter response $(2 \%)$. The total systematic uncertanity is decreasing $\sim 8 \%$ to $\sim 2 \%$ as the jet energy increases in the energy from 0 to $80 \mathrm{GeV}$ [60]. 


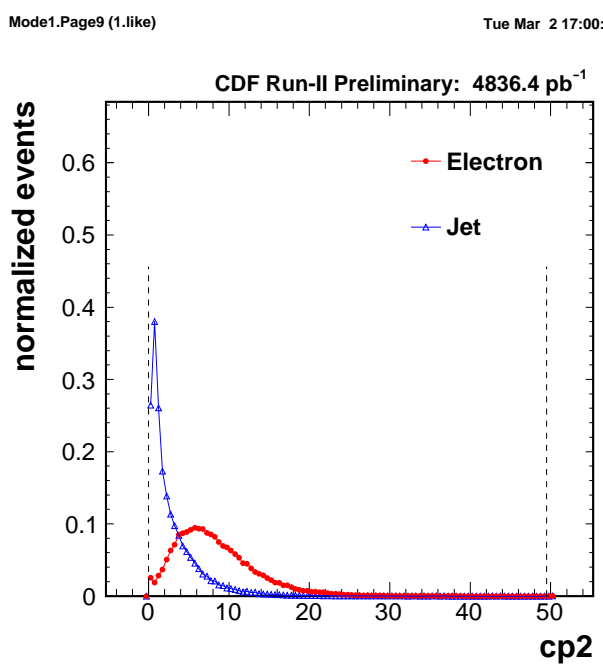

Figure 3.3: Normalized distributions of the identification variables for electrons from $Z$ decays compared to those for electron candidates in jet samples (CP2).
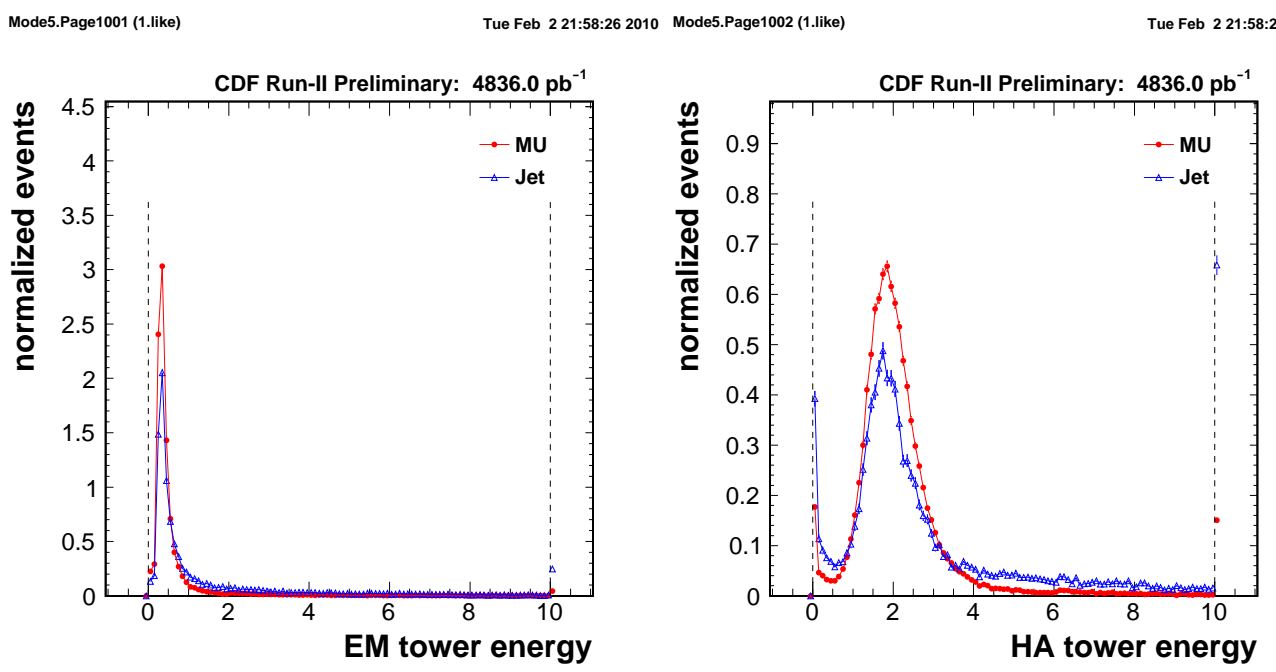

Figure 3.4: Normalized distributions of the identification variables for muons from $Z$ decays compared to those for muon candidates in jet samples.

\subsubsection{Missing Transverse Energy}

While neutrinos cannot be detected with the CDF detector, its energy will manifest as a missing energy. CDF uses "missing transverse energy $\left(\mathbb{E}_{T}\right)$ " taking into account for transverse energy imbalance because of the missing energy, the vector sum of transverse energies should be ideal null. Transverse momenta of initial particles can be neglected compared to the momenta in $z$-direction. The missing transverse energy is measured 

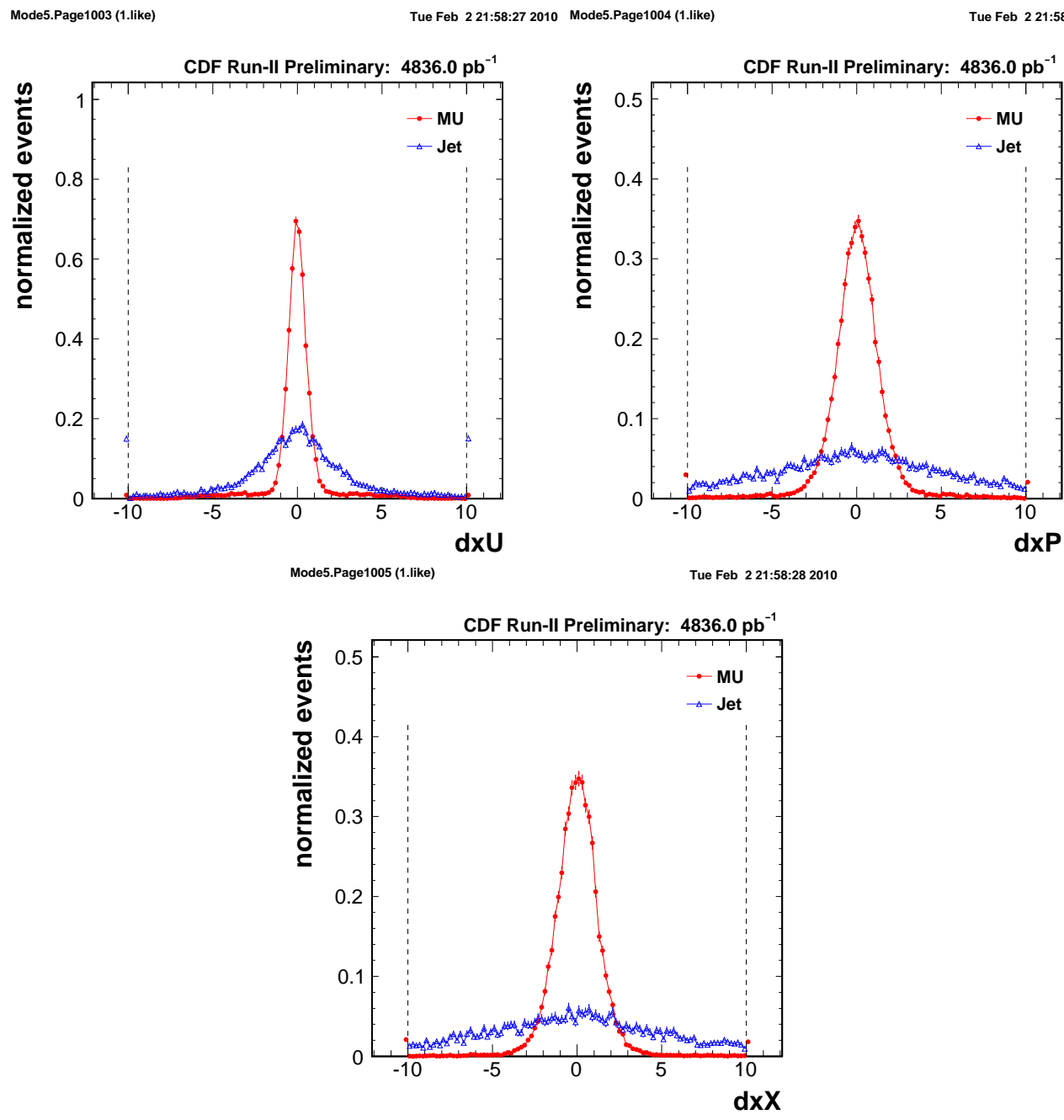

Figure 3.5: Normalized distributions of the identification variables for muons from $Z$ decays compared to those for muon candidates in jet samples (top left: $\Delta x_{\mathrm{CMU}}$, top right: $\Delta x_{\mathrm{CMP}}$, bottom: $\left.\Delta x_{\mathrm{CMX}}\right)$.

using the transverse energy imbalance,

$$
E_{T}=-\sum_{i} E_{T}^{(i)}
$$

where $E_{T}^{(i)}$ is the transverse energy of the $i$-th calorimeter tower. It needs a correction of the minimum ionization energy of a muon to the momentum measured by the track.

\subsection{Like-Sign Dilepton Event Selection}

We require asymmetric $p_{T}\left(E_{T}\right)$ requirement. The 1st lepton should have 
- electron, $E_{T}>20 \mathrm{GeV}$ and $p_{T}>10 \mathrm{GeV} / c$

- muon, $p_{T}>20 \mathrm{GeV} / c$

while, the 2nd lepton has

- electron, $E_{T}>6 \mathrm{GeV}$ and $p_{T}>6 \mathrm{GeV} / c$

- muon, $p_{T}>6 \mathrm{GeV} / c$

where the 1st lepton type is required to match trigger path, that is, when the 1st lepton is electron, the trigger path should be ELECTRON_CENTRAL_18_V*. The reason for the asymmetrical energy requirement is the trigger requirement for the 1st lepton, and 2nd lepton is much smaller than the 1st to maximize the candidate events as possible. The event selections are listed in Table 3.6.

The selected dilepton events are applied more selection cuts to clean up the sample. The additional selections are listed in Table 3.7. The dilepton is required to be consistent with coming from the same vertex, which is an important requirement for dilepton and multi-lepton signatures. The dilepton mass cut is to reject onia events such as $J / \psi$ or $\Upsilon$ (particle-antiparticle bound states). The $Z$ removal $\left(81<M_{\ell \ell}<101\right.$ $\left.\mathrm{GeV} / c^{2}\right)$ is applied to reduce $W Z$ and $Z Z$ events which potentially can be like-sign dilepton events in the final state. The $Z$-leg candidates are not the lepton passing lepton selection but also other object listed in Table 3.8 to catch $Z$ events as many as possible. Finally, like-sign charge combination requires to the dilepton events.

The number of desirable LS-dilepton events passing all event selection cuts are 624 events using the data with $9.4 \mathrm{fb}^{-1}$. 


\begin{tabular}{|c|c|c|c|}
\hline Dataset & Period & Run range & Data taking time \\
\hline Od & 0 & $138425-186598$ & 04/Feb/02 - 22/Aug/04 \\
\hline \multirow[t]{4}{*}{$\mathrm{Oh}$} & 1 & $190697-195408$ & 07/Dec/04 - 18/Mar/05 \\
\hline & 2 & $195409-198379$ & 19/Mar/05 - 20/May/05 \\
\hline & 3 & $198380-201349$ & 21/May/05 - 19/Jul/05 \\
\hline & 4 & $201350-203799$ & 20/Jul/05-04/Sep/05 \\
\hline \multirow[t]{5}{*}{$0 \mathrm{i}$} & 5 & $203819-206989$ & $05 / \mathrm{Sep} / 05-09 / \mathrm{Nov} / 05$ \\
\hline & 6 & $206990-210011$ & 10/Nov/05 - 14/Jan/06 \\
\hline & 7 & $210012-212133$ & 14/Jan/06 - 22/Feb/06 \\
\hline & 8 & $217990-222426$ & 09/Jun/06 - 01/Sep/06 \\
\hline & 9 & $222529-228596$ & 01/Sep/06 - 22/Nov/06 \\
\hline \multirow[t]{4}{*}{$0 j$} & 10 & $228664-233111$ & $24 /$ Nov/06 - 31/Jan/07 \\
\hline & 11 & $233133-237795$ & 31/Jan/07 - 30/Mar/07 \\
\hline & 12 & $237845-241664$ & 01/Apr/07 - 13/May/07 \\
\hline & 13 & $241665-246231$ & 13/May/07 - 04/Aug/07 \\
\hline \multirow[t]{4}{*}{ Ok } & 14 & $252836-254683$ & $28 /$ Oct/07 - 03/Dec/07 \\
\hline & 15 & $254800-256824$ & 05/Dec/07 - 27/Jan/08 \\
\hline & 16 & $256840-258787$ & $27 / \operatorname{Jan} / 08-27 / \mathrm{Feb} / 08$ \\
\hline & 17 & $258880-261005$ & 28/Feb/08 - 16/Apr/08 \\
\hline \multirow[t]{11}{*}{ Om } & 18 & $261119-264071$ & 18/Apr/08-01/Jul/08 \\
\hline & 19 & $264101-266513$ & 01/Jul/08 - 24/Aug/08 \\
\hline & 20 & $266528-267718$ & 24/Aug/08 - 04/Oct/08 \\
\hline & 21 & $268155-271047$ & 12/Oct/08 - 01/Jan/09 \\
\hline & 22 & $271072-272214$ & 02/Jan/09 - 10/Feb/09 \\
\hline & 23 & $272470-274055$ & 15/Feb/09 - 21/Mar/09 \\
\hline & 24 & $274123-275848$ & 22/Mar/09 - 04/May/09 \\
\hline & 25 & $275873-277511$ & 05/May/09 - 13/Jun/09 \\
\hline & 26 & $282976-284843$ & 15/Sep/09-25/Oct/09 \\
\hline & 27 & $284858-287261$ & $25 /$ Oct/09-05/Jan/10 \\
\hline & 28 & $287294-289197$ & 06/Jan/10 - 25/Feb/10 \\
\hline \multirow[t]{10}{*}{$0 p$} & 29 & $289273-291025$ & $26 / \mathrm{Feb} / 10-13 / \mathrm{Apr} / 10$ \\
\hline & 30 & $291294-293800$ & 13/Apr/10 - 19/Jun/10 \\
\hline & 31 & $293826-294777$ & 20/Jun/10 - 17/Jul/10 \\
\hline & 32 & $294778-299367$ & $21 / \mathrm{Aug} / 10-01 /$ Nov/10 \\
\hline & 33 & $299368-301303$ & 01/Nov/10 - 24/Dec/10 \\
\hline & 34 & $301952-303854$ & 06/Jan/11 - 06/Mar/11 \\
\hline & 35 & $304266-306762$ & 06/Mar/11 - 13/May/11 \\
\hline & 36 & $306791-308554$ & 13/May/11 - 04/Jul/11 \\
\hline & 37 & $308570-310441$ & 04/Jul/11 - \\
\hline & 38 & $310472-312510$ & $-30 / \operatorname{Sep} / 11$ \\
\hline
\end{tabular}

Table 3.1: List of the datasets. 


\begin{tabular}{|c|c|c|c|}
\hline ELECTRON_CENTRAL_18_v* & $\begin{array}{l}\text { L1_CEM8_PT8 } \\
\quad \cdot \text { Tower } E_{T}>8 \mathrm{GeV} \\
\quad \cdot \mathrm{HAD} / \mathrm{EM}<0.125 \\
\cdot \text { XFT } p_{T}>8.34 \mathrm{GeV} / c\end{array}$ & $\begin{array}{l}\text { L2_AUT0_L1_CEM8_PT8 } \\
\cdot \text { Auto accept. } \\
\text { L2_CEM16_PT8 } \\
\cdot \text { Cluster } E_{T}>16 \mathrm{GeV} \\
\cdot \text { HAD } / \mathrm{EM}<0.125 \\
\cdot \text { XFT } p_{T}>8 \mathrm{GeV} / c \\
\text { Added during the } 0 \mathrm{j} . \\
\cdot|\eta|<1.317 \\
\text { L2_CEM18_PT8 } \\
\cdot \text { Cluster } E_{T}>18 \mathrm{GeV}\end{array}$ & $\begin{array}{l}\text { L3_ELECTRON_CENTRAL_18 } \\
\cdot E_{T}>18 \mathrm{GeV} \\
\cdot \mathrm{HAD} / \mathrm{EM}<0.125 \\
\cdot p_{T}>9 \mathrm{GeV} / c \\
\text { Added during the 0d. } \\
\cdot L_{\text {shr }}<0.4 \\
\cdot\left|\Delta z_{\mathrm{CES}}\right|<8 \mathrm{~cm}\end{array}$ \\
\hline
\end{tabular}

Table 3.2: Main features of the ELECTRON_CENTRAL_18_v* triggers.

\begin{tabular}{|c|c|c|c|}
\hline MUON_CMUP18_v* & $\begin{array}{l}\text { L1_CMUP6_PT4 } \\
\text {. CMU } p_{T}>6 \mathrm{GeV} / c \\
\cdot \text { CMP } p_{T}>3 \mathrm{GeV} / c \\
\rightarrow \text { CMP stub (0d) } \\
\cdot \text { XFT } p_{T}>4 \mathrm{GeV} / c \\
\rightarrow 4.09 \mathrm{GeV} / c(\text { od) }\end{array}$ & $\begin{array}{l}\text { L2_AUT0_L1_CMUP6_PT4 } \\
\cdot \text { Auto accept. } \\
\text { L2_TRK8_L1_CMUP6_PT4 }(0 \mathrm{~d}) \\
\cdot \text { XFT } p_{T}>8.34 \mathrm{GeV} / c \\
\text { L2_CMUP6_PT8 (0d) } \\
\text { L2_CMUP6_PT15_3D }(0 \mathrm{j}) \\
\cdot \text { XFT } p_{T}>14.77 \mathrm{GeV} / c \\
\cdot \text { Stereo XFT }=1 \\
\text { L2_CMUP6_PT15_3DMATCH (0k) } \\
\cdot \text { Stereo XFT }=3\end{array}$ & $\begin{array}{c}\text { L3_MUON_CMUP_18 } \\
\cdot\left|d_{x}\right|_{\mathrm{U}}<10 \mathrm{~cm} \\
\cdot\left|d_{x}\right|_{\mathrm{P}}<10 \mathrm{~cm} \\
\rightarrow 20 \mathrm{~cm}(0 \mathrm{~d}) \\
\cdot p_{T}>18 \mathrm{GeV} / \mathrm{c}\end{array}$ \\
\hline MUON_CMUP18_L2_PT15_v* & L1_CMUP6_PT4 & $\begin{array}{l}\text { L2_CMUP6_PT15 } \\
\quad \cdot \text { XFT } p_{T}>14.77 \mathrm{GeV} / c\end{array}$ & L3_MUON_CMUP_18 \\
\hline MUON_CMUP18_L2_LOOSE_LUMI_260_v* & L1_CMUP6_PT4 & $\begin{array}{l}\text { L2_CMUP6_PT15_LUMI_260 } \\
\quad \cdot \text { XFT } p_{T}>14.77 \mathrm{GeV} / c \\
\quad \cdot \mathcal{L}<260 \times 10^{30} \mathrm{~cm}^{-2} \mathrm{~s}^{-1}\end{array}$ & L3_MUON_CMUP_18 \\
\hline MUON_CMUP18_L2_LOOSE_LUMI_240_v* & L1_CMUP6_PT4 & $\begin{array}{l}\text { L2_CMUP6_PT15_LUMI_240 } \\
\quad \cdot \text { XFT } p_{T}>14.77 \mathrm{GeV} / c \\
\quad \cdot \mathcal{L}<240 \times 10^{30} \mathrm{~cm}^{-2} \mathrm{~s}^{-1}\end{array}$ & L3_MUON_CMUP_18 \\
\hline MUON_CMUP18_L2_LOOSE_DPS_v* & L1_CMUP6_PT4 & $\begin{array}{l}\text { L2_CMUP6_PT15_DPS } \\
\quad \cdot \text { XFT } p_{T}>14.77 \mathrm{GeV} / c \\
\quad \text { DPS } 1-30\end{array}$ & L3_MUON_CMUP_18 \\
\hline $\begin{array}{c}\text { MUON_CMUP18_L2_PS10_L3TAG_v* } \\
\text { (p13 only) }\end{array}$ & L1_CMUP6_PT4 & $\begin{array}{l}\text { L2_CMUP6_PT15_3D_ROLXFT_PS10 } \\
\cdot \text { XFT } p_{T}>14.77 \mathrm{GeV} / c \\
\cdot \text { Stereo XFT }=1 \\
\cdot \mathrm{PS}=10\end{array}$ & L3_NULL \\
\hline
\end{tabular}

Table 3.3: Main features of the CMUP_MUON18_* triggers. 


\begin{tabular}{|c|c|c|c|}
\hline MUON_CMX18_v* & $\begin{array}{l}\text { L1_CMX6_PT8_PS1 } \\
\cdot \text { CMX } p_{T}>6 \mathrm{GeV} / c \\
\cdot \text { XFT } p_{T}>8 \mathrm{GeV} / c \\
\text { L1_CMX6_PT8_CSX_PS1 (0d) } \\
\cdot \text { CMX } p_{T}>6 \mathrm{GeV} / c \\
\cdot \text { XFT } p_{T}>8.34 \mathrm{GeV} / c \\
\cdot \text { CSX muon } \\
\text { L1_CMX6_PT8_CSX (0d) }\end{array}$ & $\begin{array}{l}\text { L2_AUT0_L1_CMX6_PT8 } \\
\text { · Auto accept. } \\
\text { L2_AUT0_L1_CMX6_PT8_CSX } \\
\text { L2_CMX6_PT10 (0d) } \\
\cdot \text { XFT } p_{T}>10.1 \mathrm{GeV} / c \\
\text { L2_CMX6_PT15_3DMATCH } \\
\cdot \text { XFT } p_{T}>14.77 \mathrm{GeV} / c \\
\text {. Stereo XFT }=3\end{array}$ & $\begin{array}{l}\text { L3_MUON_CMX18 } \\
\quad \cdot\left|d_{x}\right| \mathrm{X}<10 \mathrm{~cm} \\
\quad \cdot p_{T}>18 \mathrm{GeV} / c\end{array}$ \\
\hline MUON_CMX18_L2_PT15_v* & L1_CMX6_PT8_CSX & $\begin{array}{l}\text { L2_CMX6_PT15 } \\
\quad \cdot \text { XFT } p_{T}>14.77 \mathrm{GeV} / c\end{array}$ & L3_MUON_CMX18 \\
\hline $\begin{aligned} & \text { MUON_CMX18_L2_PT15_LUMI_200_v* } \\
& \Downarrow(0 \mathrm{i}) \\
& \text { MUON_CMX18_L2_LOOSE_LUMI_200_v* }\end{aligned}$ & L1_CMX6_PT8_CSX & $\begin{array}{l}\text { L2_CMX6_PT15_LUMI_200 } \\
\quad \cdot \text { XFT } p_{T}>14.77 \mathrm{GeV} / c \\
\quad \cdot \mathcal{L}<200 \times 10^{30} \mathrm{~cm}^{-2} \mathrm{~s}^{-1}\end{array}$ & L3_MUON_CMX18 \\
\hline MUON_CMX18_L2_LOOSE_DPS_v* & L1_CMX6_PT8_CSX & $\begin{array}{l}\text { L2_CMX6_PT15_DPS } \\
\quad \cdot \text { XFT } p_{T}>14.77 \mathrm{GeV} / c \\
\quad \text {. DPS } 1-80\end{array}$ & L3_MUON_CMX18 \\
\hline MUON_CMX18_LUMI_250_v* & L1_CMX6_PT8_CSX & $\begin{array}{l}\text { L2_CMX6_PT15_3D_LUMI_250 } \\
\cdot \text { XFT } p_{T}>14.77 \mathrm{GeV} / c \\
\cdot \text { Stereo XFT } \\
\cdot \mathcal{L}<250 \times 10^{30} \mathrm{~cm}^{-2} \mathrm{~s}^{-1}\end{array}$ & L3_MUON_CMX18 \\
\hline MUON_CMX18_DPS_v* & L1_CMX6_PT8_CSX & $\begin{array}{l}\text { L2_CMX6_PT15_3D_DPS } \\
\cdot \text { XFT } p_{T}>14.77 \mathrm{GeV} / c \\
\cdot \text { Stereo XFT } \\
\text {. DPS } 1-10\end{array}$ & L3_MUON_CMX18 \\
\hline MUON_CMX18_L2_PS100_L3TAG_V* & L1_CMX6_PT8_CSX & $\begin{array}{l}\text { L2_CMX6_PT15_3D_ROLXFT_PS100 } \\
\cdot \text { XFT } p_{T}>14.77 \mathrm{GeV} / c \\
\cdot \text { Stereo XFT }=1 \\
\quad \cdot \mathrm{PS}=100\end{array}$ & L3_MUON_CMX18 \\
\hline MUON_CMX18_L2_PS10_v* & L1_CMX6_PT8_CSX & $\begin{array}{l}\text { L2_CMX6_PT15_3D_PS10 } \\
\quad \cdot \text { XFT } p_{T}>14.77 \mathrm{GeV} / c \\
\cdot \text { Stereo XFT }=1 \\
\quad \cdot \mathrm{PS}=10\end{array}$ & L3_MUON_CMX18 \\
\hline MUON_CMX18_L2_PS100_v* & L1_CMX6_PT8_CSX & $\begin{array}{l}\text { L2_CMX6_PT15_3D_PS100 } \\
\cdot \text { XFT } p_{T}>14.77 \mathrm{GeV} / c \\
\cdot \text { Stereo XFT }=1 \\
\quad \cdot \mathrm{PS}=100\end{array}$ & L3_MUON_CMX18 \\
\hline
\end{tabular}

Table 3.4: Main features of the CMX_MUON18_* triggers. 


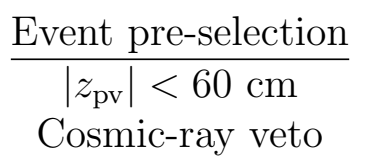

Geometrical and kinematical cuts

CEM

Fiducial

$E_{T}^{\ell_{1}}>20 \mathrm{GeV}\left(p_{T}>10 \mathrm{GeV} / c\right)$

$E_{T}^{\ell_{2}}>6 \mathrm{GeV}\left(p_{T}>6 \mathrm{GeV} / c\right) \quad p_{T}^{\ell_{2}}>6 \mathrm{GeV} / c$
CMUP, CMX, CMU, CMP, BMU,

CMIO-CES, or CMIO-PES

Fiducial, $\rho_{\mathrm{COT}}>140 \mathrm{~cm}(\mathrm{CMX})$

Blue-beam veto, keystone veto, miniskirt veto

$p_{T}^{\ell_{1}}>20 \mathrm{GeV} / c$

$$
\begin{gathered}
\text { Axial } \geq 3 \text { and stereo } \geq 3(\geq 7 \text { hits }) \\
\left|z_{0}-z_{\text {pv }}\right|<2 \mathrm{~cm} \\
\text { Silicon hits } \geq 3 \\
\left|d_{0}\right|<0.02 \mathrm{~cm}
\end{gathered}
$$

Isolation cut

$\mathrm{ISO}_{0.4}^{\mathrm{cal}}<2 \mathrm{GeV}$

(Likelihood value) $\geq 0.70$

$\underline{\text { Identification cuts }}$

(Likelihood value) $\geq 0.20$ (CMUP and CMX),

(Likelihood value) $\geq 0.90$ (other muons)

Conversion removal

Other cuts

Table 3.6: Event pre-selection and lepton selection cuts.

\begin{tabular}{l}
\hline \hline Exactly two leptons \\
$\left|z_{0}^{\ell_{1}}-z_{0}^{\ell_{2}}\right|<2 \mathrm{~cm}$ \\
Dilepton mass $>12 \mathrm{GeV} / c^{2}$ \\
$Z$ removal \\
At least one like-sign pair \\
\hline \hline
\end{tabular}

Table 3.7: Dilepton selection cuts. 


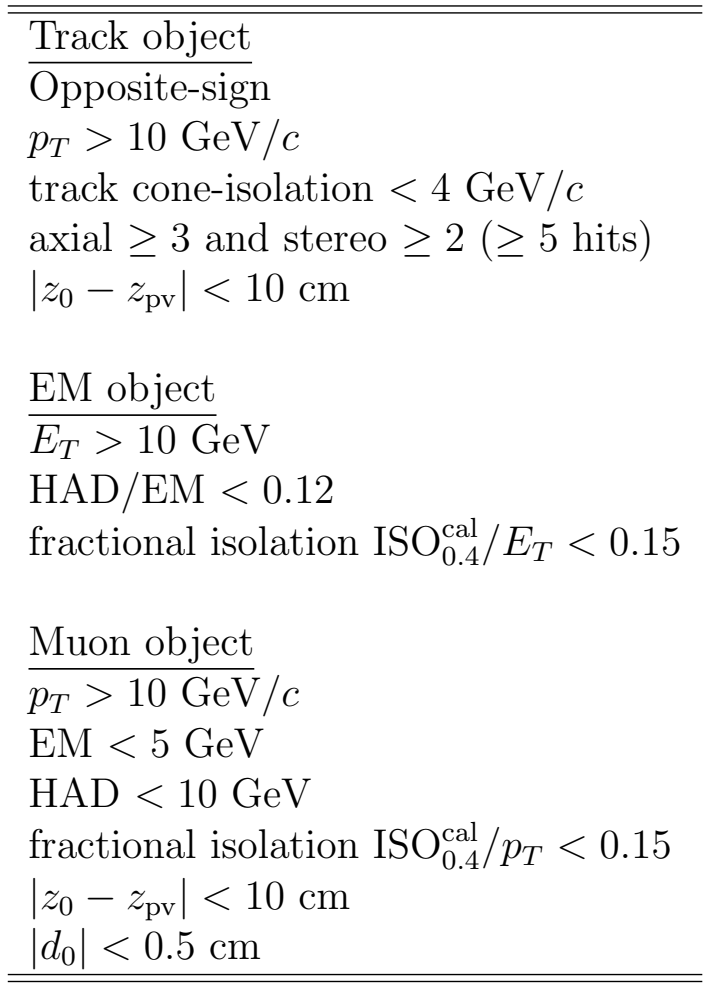

Table 3.8: Physics objects used to identify and remove $Z$ bosons. 



\section{Chapter 4}

\section{Background Estimation}

Although the like-sign requirement is quite effective to 164 suppress QCD and known electroweak processes, we expect that fake-lepton backgrounds and residual photon conversions still remain at a considerable level. They are estimated by data-driven methods, while other backgrounds containing prompt real-leptons (physics backgrounds) are estimated using Monte Carlo samples.

\subsection{Residual Photon-Conversions}

\subsubsection{Photon-Conversion Detection Efficiency}

Once we know the detection efficiency, $\varepsilon_{\text {con }}$, we can calculate a ratio of the residual conversions to the identified conversions, $R_{\text {res }}$, by

$$
R_{\mathrm{res}}=\frac{1-\varepsilon_{\mathrm{con}}}{\varepsilon_{\mathrm{con}}}
$$

The amount of residual conversions in samples of our interest can be basically estimated by multiplying $R_{\text {res }}$ to the number of identified conversions. The detection efficiency is defined by taking the denominator to be conversions with one leg passing our electron selection criteria except the isolation cut and conversion veto, which we refer to as the seed electron. The reason for turning off the isolation cut is rather technical. We use fake-event Monte Carlo (MC) samples created by generating single $\gamma$ or $\pi^{0}$ to compare conversions with those in the real data, and the isolation variable in such fake-events is not usable in this respect.

The base efficiency is estimated as a function of partner-track $p_{T}$ and decomposed into two factors in our analysis as

$$
\varepsilon_{\mathrm{con}}\left(p_{T}\right)=\varepsilon_{\mathrm{rel}}\left(p_{T}\right) \cdot \varepsilon_{\mathrm{pla}},
$$

where $\varepsilon_{\text {rel }}\left(p_{T}\right)$ is the $p_{T}$ dependent efficiency covering low- $p_{T}$ regions and defined relative to the efficiency on the plateau, and $\varepsilon_{\text {pla }}$ is the absolute efficiency on the plateau. 
These efficiencies could be further broken down to the track-finding efficiency and the efficiency of the conversion identification criteria given by

$$
\left|\delta_{x y}\right|<0.2 \mathrm{~cm} \text { and }|\Delta(\cot \theta)|<0.04,
$$

where $\left|\delta_{x y}\right|$ is the distance of the closest approach points between a pair of tracks of conversion candidates. We need to clarify that our conversions are those for which the finder function returns the flag to 1, and the so-called tridents which have a third track with the same charge as the seed electron are not included since they are likely to be prompt electrons.

The inefficiency of detecting conversions is dominated by that of reconstructing low momentum partner-tracks and manifests itself in $\varepsilon_{\text {rel }}\left(p_{T}\right)$. It is not negligible even for high- $E_{T}$ seed electrons because the kinematics of conversions relatively favors asymmetric energy sharing as shown in Figure 4.1 which is a theoretical calculation of the relative probability of energy sharing as a function of fractional electron energy [61]. Its asymmetric nature enhances the residual conversion events.

Our first goal is to measure the base efficiency, $\varepsilon_{\text {rel }}\left(p_{T}\right)$, by comparing observed $p_{T}$ spectrum of identified conversions to a perfect, or generator-level, MC spectrum. It turns out, however, that direct uses of this efficiency would not be useful in actual applications because it includes very low values at low $p_{T}$ region. From the form of the residual conversion ratio, $(1-\varepsilon) / \varepsilon$, too-low efficiencies in a certain parametrization results in a significant loss of statistical power of predicting residual conversions. In other words, we are forced to estimate the most important part of residual conversions using the identified conversions with the least statistics. In order to avoid this problem, we transform the base efficiency to a function of different parameter, and we choose the parent-photon $p_{T}$ for such parametrization. The base efficiency as a function of partner-track $p_{T}$ is convoluted with the perfect $p_{T}$ spectra of the $\mathrm{MC}$ for a given $p_{T}$ bin of the parent photon to get $\varepsilon_{r e l}^{\gamma}\left(p_{T}\right)$.

The next is to measure the plateau efficiency, $\varepsilon_{\text {pla }}$, by using conversions in the real data identified with a method independent from our nominal identification algorithm.

Finally, the $\varepsilon_{c o n}^{\gamma}\left(p_{T}\right)$ is obtained by multiplying this absolute efficiency to the relative efficiency:

$$
\varepsilon_{\mathrm{con}}^{\gamma}\left(p_{T}\right)=\varepsilon_{\mathrm{rel}}^{\gamma}\left(p_{T}\right) \cdot \varepsilon_{\mathrm{pla}} .
$$

\subsubsection{Data Samples}

\section{$\underline{\text { Real Data }}$}

We use the bhel* dataset. We apply a pre-selection including bad-run removal using a list of the version vhiggs with "no Si" and "CMX ignored" options, and a trigger bit selection of ELECTRON_CENTRAL_18_v*. The sample corresponds to an integrated luminosity of $9.7 \mathrm{fb}^{-1}$. From these samples, we select events with at least one good electron satisfying our high- $p_{T}$ electron selection $\left(E_{T}>20 \mathrm{GeV}\right.$ and $\left.p_{T}>10 \mathrm{GeV} / c\right)$ except for the isolation cut and the conversion veto. 


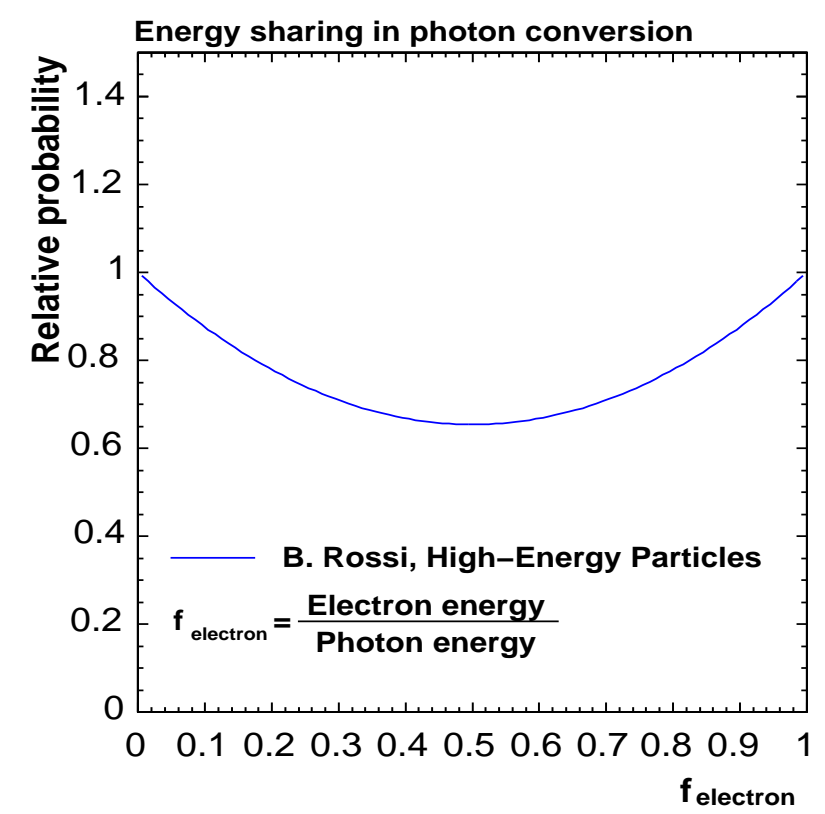

Figure 4.1: A theoretical calculation of the relative probability of energy sharing in the photon conversion as a function of fractional electron $\left(e^{-}\right)$energy [61].

\section{Conversion Monte Carlo Samples}

There are four MC-samples used in this study. The two of them are made by generating events of single $\pi^{0}$ using the FAKE_EVENT module: one with a flat $p_{T}$ spectrum and the other with a certain $p_{T}$ slope. The rest of the samples are made by generating single- $\gamma$ events instead of $\pi^{0}$. The generation parameters are listed in Table 4.1. The "Good" in the table means that there is at least one high- $p_{T}$ good electron in the event. Simulations of $\pi^{0}$ decays and succeeding conversions of $\gamma$ themselves are left to the CDF detector simulation package, cdfSim, of the offline version 5.3.3. The simulated probability of conversion is about $18 \%$ per photon independent of its $p_{T}$.

\begin{tabular}{cccccccr}
\hline \hline Sample & Particle & $\begin{array}{c}\min -p_{T} \\
(\mathrm{GeV} / c)\end{array}$ & $\begin{array}{c}\max -p_{T} \\
(\mathrm{GeV} / c)\end{array}$ & $\begin{array}{c}\text { Slope } \alpha \\
\left(p_{T}^{-\alpha}\right)\end{array}$ & max- $|\eta|$ & $\begin{array}{c}\text { Generated } \\
\text { (events) }\end{array}$ & $\begin{array}{c}\text { Good } \\
\text { (events) }\end{array}$ \\
\hline 1. & $\pi^{0}$ & 3 & 150 & 0 (flat) & 1.3 & 1520000 & 227151 \\
2. & $\pi^{0}$ & 15 & 150 & 6.3 & 1.3 & 1600000 & 188012 \\
3. & $\gamma$ & 3 & 150 & 0 (flat) & 1.3 & 1580000 & 140580 \\
4. & $\gamma$ & 15 & 150 & 4.8 & 1.3 & 1580000 & 136945 \\
\hline \hline
\end{tabular}

Table 4.1: Monte Carlo samples. 
Since $p_{T}$ spectra of partner tracks are correlated with $p_{T}$ of parent photons, we need to match MC slopes with those we observe in the data. We note here that the slopes in Table 4.1 are not necessarily the optimized ones but the values we pick up for reasons nothing more than historical. In the $\mathrm{MC}$ tuning, we try to describe conversions found in the data with a mixture of the $\pi^{0}$ and $\gamma$ MCs with optimized slopes. In order to improve the statistical power of the MCs in the optimization, the "flat samples" (the samples 1 and 3 in Table 4.1) are used to optimize the slopes, while the samples generated with the slopes in the table are combined appropriately, after re-weighting with the tuned slopes, to find out the optimum relative ratio $\gamma / \pi^{0}$.

\subsubsection{Monte Carlo Tuning}

Tuning the MC samples is done by using identified conversions collected with an additional requirement of partner-track $p_{T}>2 \mathrm{GeV} / c$ which is expected to be high enough to avoid the non-plateau region. The slope parameter $\alpha$ is optimized by matching the weighted $p_{T}$ spectra of reconstructed photon with what is observed in the real data, where the photon $p_{T}$ is formed by the scalar- $p_{T}$ sum of the seed-electron track and the partner track. On the other hand, we use $E / p$ distributions to constrain the mixture of the $\pi^{0}$ and $\gamma$. The actual optimization of the three parameters, two slopes for the $\pi^{0}$ and $\gamma$, and the relative ratio $\gamma / \pi^{0}$, is carried out by an iterative way. Namely, 1) find a relative ratio for a given set of two slopes; 2) by adding the $\pi^{0}$ and $\gamma$ MCs with the obtained ratio, we look for the best set of two slopes that describes photon $p_{T}$ of the data using $\left.\chi^{2} ; 3\right)$ go back to the first step and iterate. The optimized parameters are

$$
\alpha_{\pi^{0}}=5.5, \quad \alpha_{\gamma}=5.0, \quad \gamma / \pi^{0}=0.68 \pm 0.04
$$

Figure 4.2 shows the optimized $E / p$ distribution compared to the data. The reduced $\chi^{2}$ as a function of slope parameters is shown in Figure 4.3. Figure 4.4 shows a comparison of reconstructed photon- $p_{T}$ spectra between the $\mathrm{MC}$ and the data. We also demonstrate that our MC samples simulate the conversion points reasonably well in Figure 4.5, and that the detailed $p_{T}$ and $E_{T}$ spectra of seed electrons are in good agreement with the data as shown in Figures 4.6 and 4.7. The jump at $50 \mathrm{GeV}$ seen in Figure 4.7 is due to the $E / p$ cut turned off at this $E_{T}$. From these figures, we say that our MC events reproduce observed conversions well in a wide kinematical range.

\subsubsection{Relative Efficiency in Low $p_{T}$ Region}

Figure 4.8 compares the $p_{T}$ spectrum of conversion partner-tracks observed in the real data with the one obtained at the generator level of MC, where the MC spectrum is fit to the data in the region of $2<p_{T}<5 \mathrm{GeV} / c$. We clearly see losses of tracks in very low- $p_{T}$ region. The efficiency relative to the plateau, $\varepsilon_{\text {rel }}\left(p_{T}\right)$, is obtained by dividing the observed partner-track distribution by the fitted MC distribution, and is shown in Figure 4.9, where the uncertainties in the figure are only statistical. The efficiency 
Mode103.Page16 (1.conv)

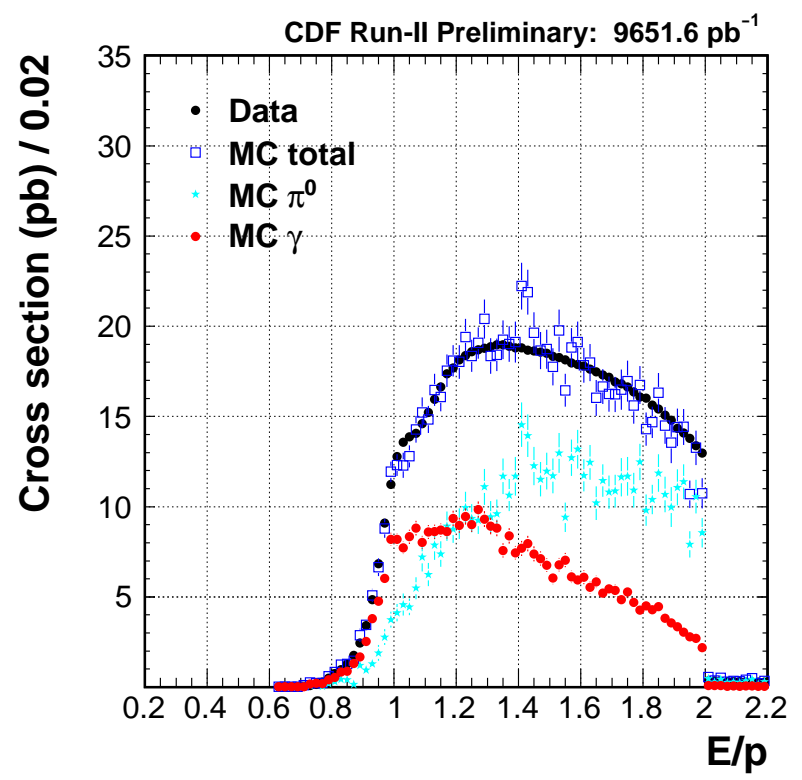

Figure 4.2: Distribution of $E / p$ for the seed electron of conversions.

Mode103.Page8 (1.conv)

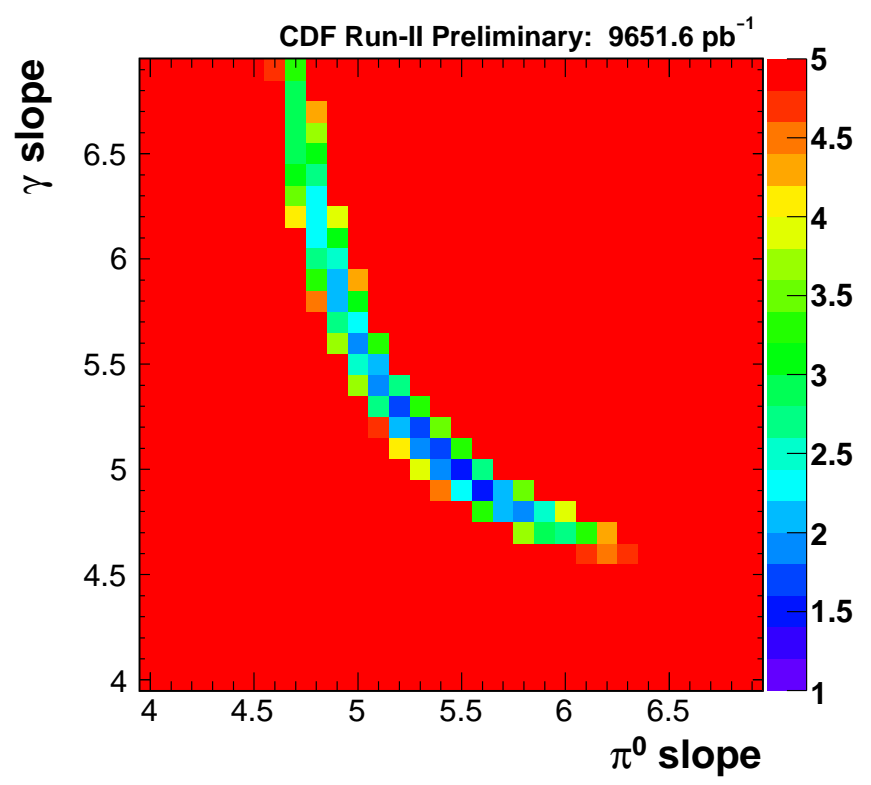

Figure 4.3: Reduced $\chi^{2}$ between the data and the Monte Carlo distributions of reconstructed photon- $p_{T}$ as a function of Monte Carlo slope-parameters. 
Mode103.Page12 (1.conv)

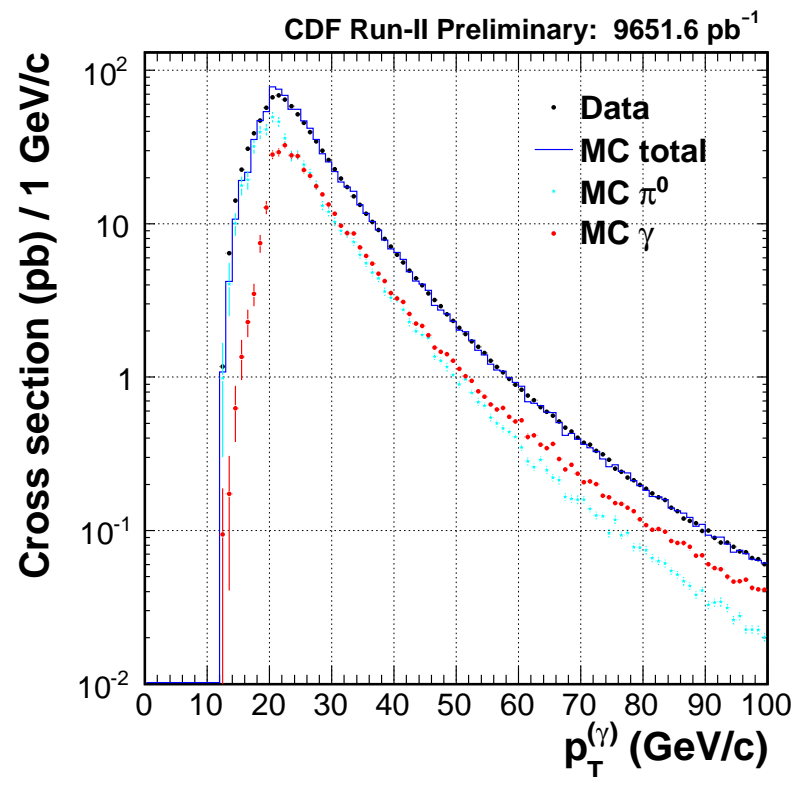

Figure 4.4: The reconstructed photon- $p_{T}$ spectrum.

Mode107.Page7 (1.conv)

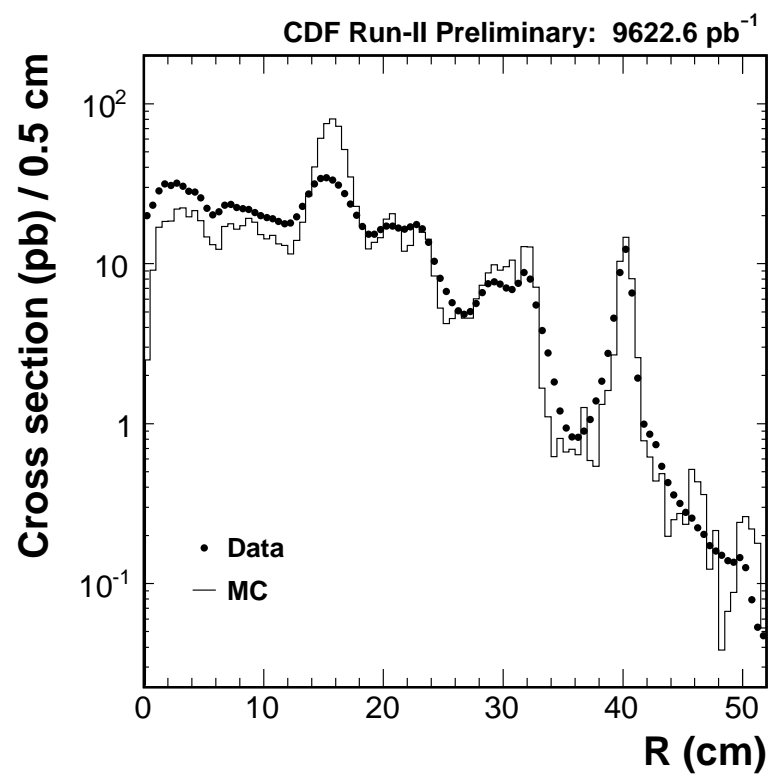

Figure 4.5: Distribution of the radius of conversion points from the beam line. 
Mode103.Page28 (1.conv)

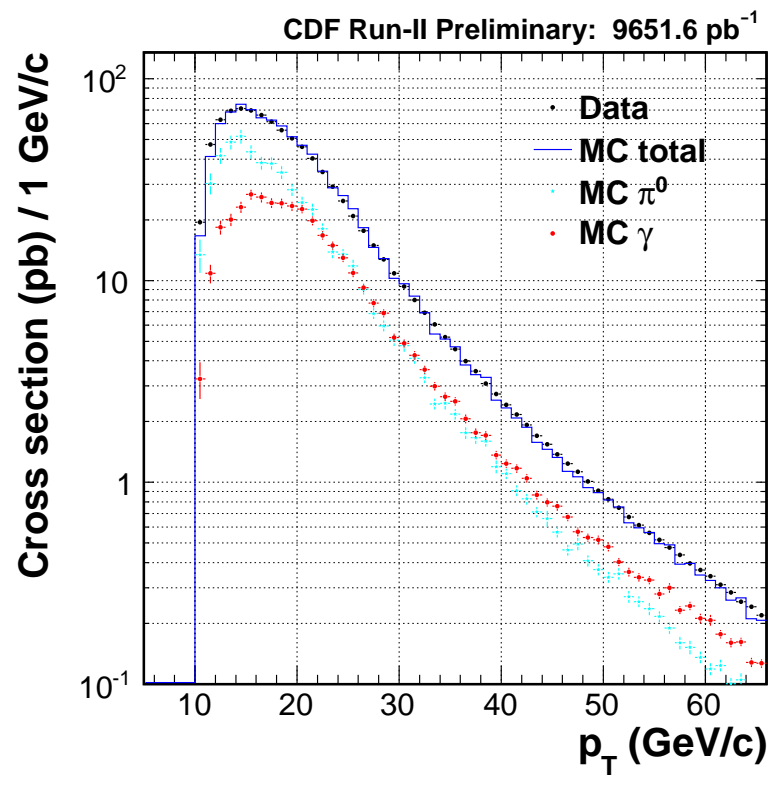

Figure 4.6: Conversion-electron $p_{T}$ spectrum.

Mode103.Page9 (1.conv)

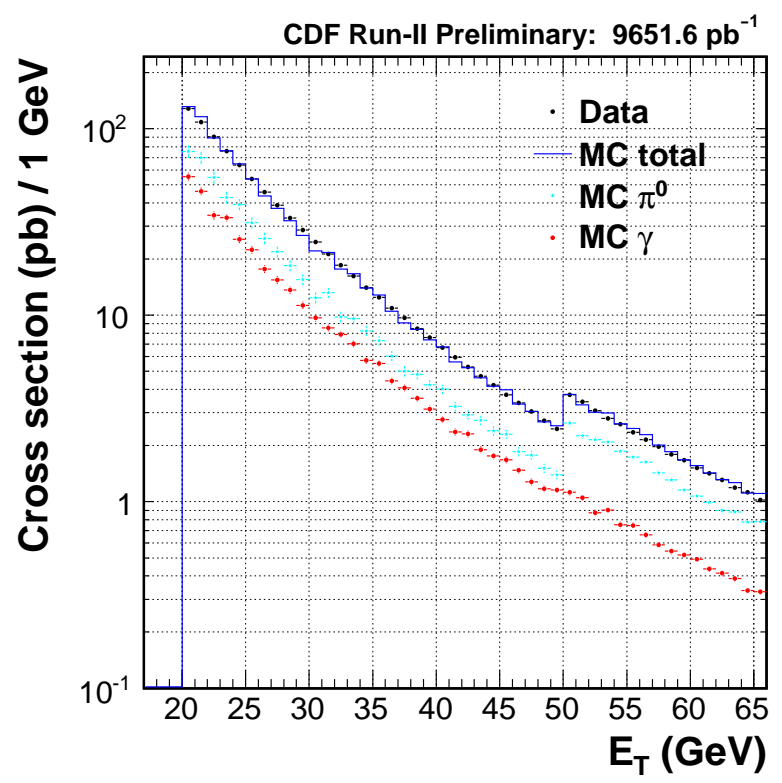

Figure 4.7: Conversion-electron $E_{T}$ spectrum.

reaches its plateau at $p_{T} \simeq 1 \mathrm{GeV} / c$. Figure 4.9 alsoshows the $\mathrm{MC}$ prediction of the efficiency. The MC performance is actually good in reproducing the $p_{T}$ dependence of 
the conversion-detection efficiency.

We investigate systematic uncertainties of the relative efficiency coming from the MC tuning parameters. This is done by shifting the parameters by the quoted uncertainties given in Eq. 4.5, and the result is summarized in Figure 4.10 in terms of relative uncertainties. The uncertainty is dominated by $\mathrm{MC}$ statistics and at a level of $5 \%$. The dependence on the MC tuning is small; only the slope dependence becomes non-negligible at very low $p_{T}$.

The relative efficiency as a function of partner-track $p_{T}$ is an important and the most basic efficiency, but it is not useful in actual applications. Residual conversions result from low efficiencies of detecting conversions, which in turn means that the statistical power of identified conversions is low as well. If we use the parametrization of the partner-track $p_{T}$, we would suffer from it to a considerable extent because there is an extremely low efficiency point, and have to predict the most contributing residual conversions from the least statistics of identified conversions. A solution is to choose a different parametrization so that low and high efficiency regions are reasonably mixed and smeared out.

One might think that natural choices would be the $E_{T}$ or $p_{T}$ of seed electrons because the seed electron is a physics object we identify primarily in physics analyses. However, we found that such parametrizaitons had sample-to-sample dependences through different photon- $p_{T}$ spectra. As an example, we consider the parametrization using the seed-electron $E_{T}$.

Before proceeding to an investigation of parametrization, we note that the transformation to a different parametrization is done by using only the base relative efficiency which has been just measured and the generator-level MC information regarding the partner-track $p_{T}$ spectrum. The $p_{T}$ cut on the seed-electron no longer has to be high in this situation because the generated $\mathrm{MC}$ particles cover low- $p_{T}$ regions down to 3 $\mathrm{GeV} / c$ as given in Table 4.1. The kinematical cut in the actual like-sign dilepton analysis is asymmetric, where the low- $p_{T}$ cut is defined to be $E_{T}>6 \mathrm{GeV}$ and $p_{T}>6$ $\mathrm{GeV} / c$, and the primary estimation of residual conversion backgrounds in the like-sign dilepton analysis will be carried out for this low- $p_{T}$ side of dilepton pairs. Given this, we mainly discuss the relative efficiency with different parametrizations by imposing the low- $p_{T}$ cut in what follows.

For getting the relative efficiency as a function of seed-electron $E_{T}$, we convolute the base efficiency as a function of partner-track $p_{T}$ with the generator-level spectrum of partner-track $p_{T}$ in a given seed-electron $E_{T}$ bin. In this case, the partner-track $p_{T}$ spectrum is inevitably dependent on the parent photon- $p_{T}$ spectrum which has basically a sample dependence. In fact, a comparison of photon- $p_{T}$ spectra between the $\mathrm{MC}$ that is tuned by the inclusive electron data and like-sign lepton + conversion events shown in Figure 4.11 demonstrates such a dependence. We see that a broad $p_{T}$ spectrum is favored in the (lepton + conversion) events. Our analysis shows that the $p_{T}$ spectrum of $\propto p_{T}^{-2.5}$ of the $\gamma \mathrm{MC}$ describes the observed spectrum better, which should be compared to $p_{T}^{-4.9}$ tuned by the inclusive electron sample. The relative efficiency as a function of electron $E_{T}$ for each case is shown in Figure 4.12. There is a $\simeq 15 \%$ 
difference at a lower $E_{T}$ regions, which translates to about a $40 \%$ change in terms of the residual conversion ratio as calculated from

$$
\frac{\Delta R_{\mathrm{res}}}{R_{\mathrm{res}}}=-\frac{1}{1-\varepsilon} \frac{\Delta \varepsilon}{\varepsilon} .
$$

We could measure the slopes for samples of interest but it is not always possible due to, for example, limited statistics.

In order to obtain more stable efficiencies over different samples, we choose to use the parent-photon $p_{T}$ itself to parametrize them. The convolution is expressed by

$$
\varepsilon_{\mathrm{rel}}^{\gamma}\left(p_{T}\right)=\frac{\int f\left(\tilde{p}_{T} \mid p_{T}\right) \cdot \varepsilon_{\mathrm{rel}}\left(\tilde{p}_{T}\right) d \tilde{p}_{T}}{\int f\left(\tilde{p}_{T} \mid p_{T}\right) d \tilde{p}_{T}}
$$

where $f\left(\tilde{p}_{T} \mid p_{T}\right)$ represents the true (i.e. generator level) $p_{T}$ spectrum of partner tracks for a given $p_{T}$ of the parent photon. In actual calculation, the $\varepsilon_{\text {rel }}\left(p_{T}\right)$ for the tracks with $p_{T}>2 \mathrm{GeV} / c$ (normalization region) is set to 1 . The obtained relative efficiency as a function of photon $p_{T}$ is shown in Figure 4.13, where the uncertainties are statistical only. The drop of the efficiency for the $p_{T}$ less than $15 \mathrm{GeV} / c$ is a threshold effect and it does not mean that the intrinsic efficiency is low in this $p_{T}$ region. In the figure, efficiencies from $\gamma$ and $\pi^{0}$ MCs are separately shown, and they are consistent with each other. This gives us an advantage that the application is not sensitive to the composition of $\gamma$ and $\pi^{0}$ in samples. Related to this, we show a comparison of $E / p$ distributions for conversions in the (like-sign lepton + conversion) events, the $\gamma \mathrm{MC}$, and the $\pi^{0} \mathrm{MC}$ in Figure 4.14, where the MC slope-parameters are tuned by the (likesign lepton + conversion) events themselves and the $\mathrm{MC}$ distributions are normalized to the data. We should comment that the conversions found in the (like-sign lepton + conversion) events are more likely to be originating from photons, not from $\pi^{0}$. In fact, if we try to fit the data with the two MC distributions, a fitter returns answers of negligible contributions from the $\pi^{0} \mathrm{MC}$. From now on, we use the efficiency obtained by using the $\gamma$ MC.

We mention here one complication in the calculation of statistical uncertainties included in Figure 4.13. For a given $p_{T}$ bin of the parent photon, the denominator shows a certain $p_{T}$ distribution of partner tracks. This denominator is a sum of weights related to the MC slope. The numerator accepts another weight which is the relative efficiency $\varepsilon_{\text {rel }}\left(p_{T}\right)$. The efficiency for a given $p_{T}$ bin is thus symbolically written as

$$
\varepsilon=\frac{\sum w_{i}^{\prime} w_{i} x_{i}}{\sum w_{i} x_{i}}
$$

where $x_{i}$ is actually 1 (event) for all $i$. The statistical uncertainty of $\varepsilon$ is basically calculated by propagating the uncertainty of $x_{i}$, namely $\Delta x_{i}=1$ :

$$
\Delta \varepsilon=\frac{\sum\left(w_{i}^{\prime} w_{i}-\varepsilon w_{i}\right) \Delta x_{i}}{\sum w_{i} x_{i}}=\frac{\sum\left(w_{i}^{\prime} w_{i}-\varepsilon w_{i}\right)}{D}
$$


where $D=\sum w_{i} x_{i}$ is the integral of entries in the denominator. From this equation, we see that there arises a cross term proportional to $w^{\prime} w^{2}$ when we compute $\left\langle(\Delta \varepsilon)^{2}\right\rangle$. We thus need to accumulate the square of weights of the form $\sqrt{w^{\prime}} w$ in order to calculate the statistical uncertainty properly. As the systematic uncertainty of the relative efficiency as a function of photon $p_{T}$, we consider the input relative efficiency. Shifting them by $\pm \sigma$, we look at resulting changes of the final efficiency. A summary of the uncertainty calculations is shown in Figure 4.15, where the total uncertainty is obtained by adding all uncertainties in quadrature.

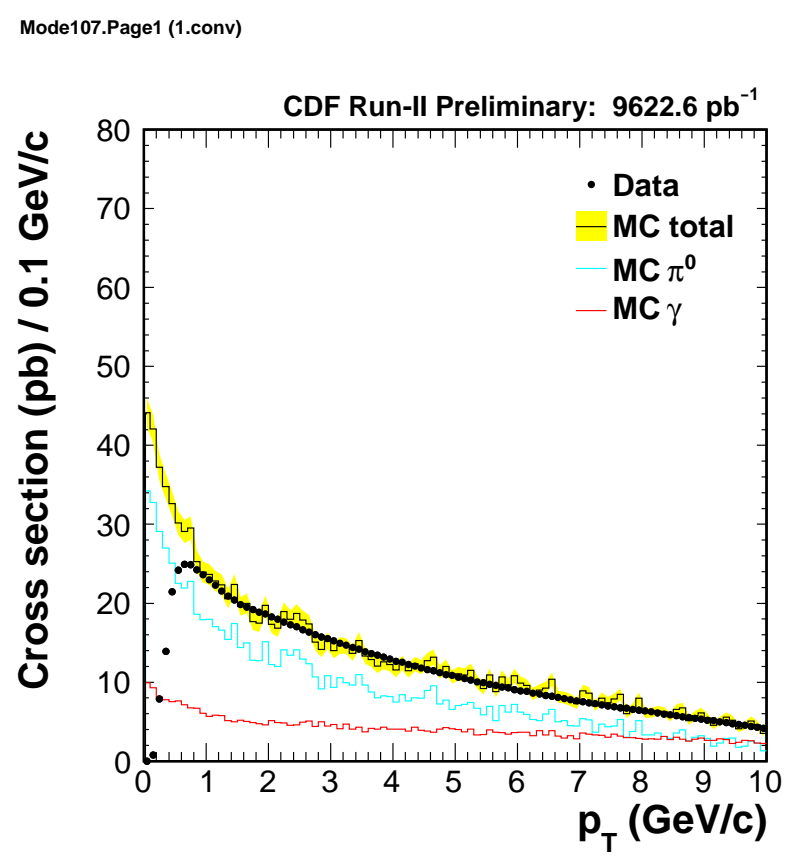

Figure 4.8: Partner-track $p_{T}$ spectrum for conversions. The Monte Carlo spectrum is shown at the generator (OBSP) level and fitted to the data in the $p_{T}$ region $2<p_{T}<5$ $\mathrm{GeV} / c$.

\subsubsection{Absolute Efficiency on the Plateau}

We need to identify conversions with a method independent from the nominal identification algorithm to measure the absolute efficiency on the plateau region. By the same way as the previous analysis, we use hit information of the CES strips for this purpose. It is based on a distinct feature of the conversion pair that the electron and the positron have approximately the same $z$ position at any radius. When the electron and the positron separate enough so that they reach different $\phi$ wedges as illustrated in Figure 4.16, we can measure each $z$ position of the electron and the positron on the CES plane, and will find that the $z$ position difference, $\Delta z$, has a peak around zero in the "right" side (RS) wedge as expected from the charge combination. We refer 


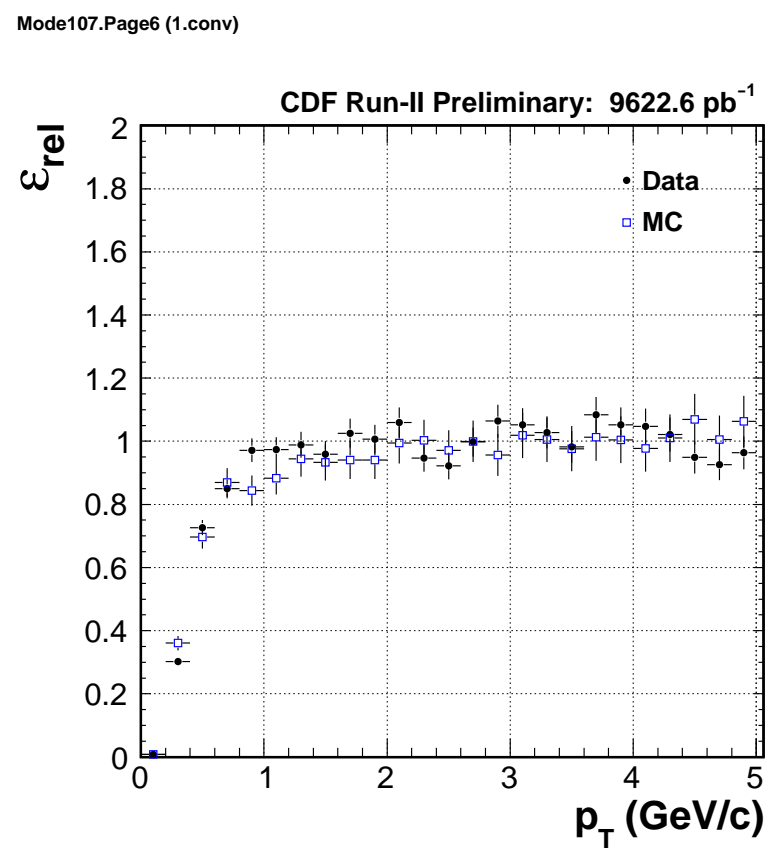

Figure 4.9: Relative conversion-detection efficiency as a function of partner-track $p_{T}$. Only statistical uncertainties are shown.

to this identification of conversions as the CES method. In our analysis, we look for the highest energy CES cluster in the nearest three towers along the $\eta$ direction of the wedges next to the seed electron (see Figure 4.17).

We use high- $p_{T}$ electron samples described in Section 4.1.2 to measure the plateau efficiency. In order to make the geometrical configuration of conversions clear, we here impose a set of "fiducial" cuts on the electrons, which essentially selects only the electrons located in the central region inside a tower. One requirement is that the local $x$ coordinate of the extrapolated track at the CES plane satisfy $|x|<15 \mathrm{~cm}$. Another cuts is for the $z$ direction to ensure that the partner track of a conversion is hitting the tower with the same $\eta$-index as the seed-electron tower. The appropriate definition of the $z$ fiducial is obtained by looking again at identified conversions, but this time, only those with the $\eta$ indices being different between the seed-electron tower and the tower the partner track is pointing. Figure 4.17 shows a distribution of the un-signed detector $\eta$ for the seed electrons with such mismatching tower indices, where the partner-track $p_{T}$ is required to be larger than $1 \mathrm{GeV} / c$, that is, in the plateau region. We avoid the $\eta$ regions seen as peaks in the figure. Then, it is natural and relevant to require the CES cluster that we have found belong to the tower with the same $\eta$-index as the electron, that is, exactly next to the electron tower and in the RS wedge. The last of our "fiducial" cuts is to require the number of tracks associated with the electron be just one in order to suppress the conversions with the partner track passing through the same tower as the seed electron, which ensures better configuration we desire. 


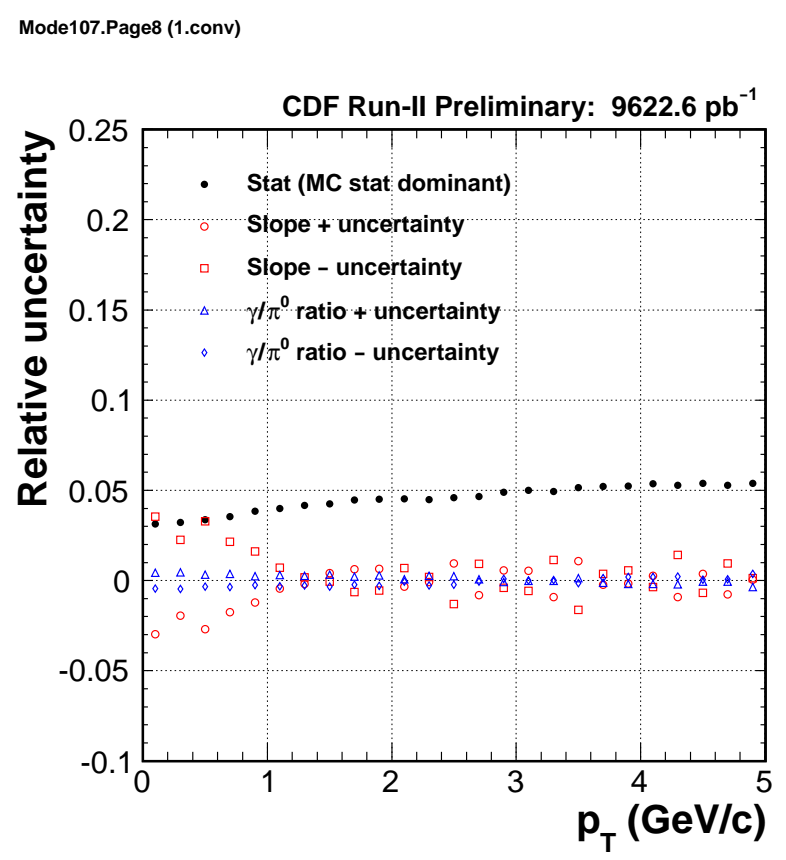

Figure 4.10: Relative uncertainty of the relative conversion-detection efficiency originating from different sources.

Finally, we demand a certain energy deposition in the RS tower to purify the sample: $E_{\text {strip }} / p>0.5$ and $E_{\mathrm{CEM}} / p>0.7$, where the track momentum $p$ is calculated from the assumed $p_{T}$ of $2 \mathrm{GeV} / c$ and the $z$ position of the CES cluster. The requirements introduced above are applied only to the information of the electrons and the RS tower, and none is explicitly required on anything of partner tracks.

A demonstration of the $\Delta z$ peaks for the RS and the wrong side (WS) wedge is given in Figure 4.18 in which we see a clear separation between what we observe in the RS and in the WS. The bremsstrahlung emitted off an electron could produce a false $\Delta z$ peak in the RS tower but the probability of such cases is confirmed to be very small, less than $0.1 \%$, by using a large MC sample of single electrons.

The distribution is fit with Gaussian plus constant. The absolute efficiency is measured by looking at the reduction of the fitted Gaussian functions before and after our nominal conversion identification algorithm. The result is shown in Figure 4.19. The ratio of the background to the signal (Gaussian) part is about $20 \%$ and $10 \%$ before and after the conversion identification, respectively. The efficiency is obtained to be $\varepsilon_{\text {pla }}=0.926 \pm 0.002$. Instead of using the fitted Gaussian functions, we also try to count the excess events over the fitted background, which gives $\simeq 2 \%$ larger counts. The efficiency in this case is, however, still stable and found to be $0.94 \pm 0.01$. The contamination of low-momentum partner-tracks with $p_{T}<1 \mathrm{GeV} / c$ in the $\Delta z$ peak is checked using the sample after the conversion tagging cut and found to be small (1\%). We do not observe any large systematics in the $E_{T}$ dependence but just fluctuations 


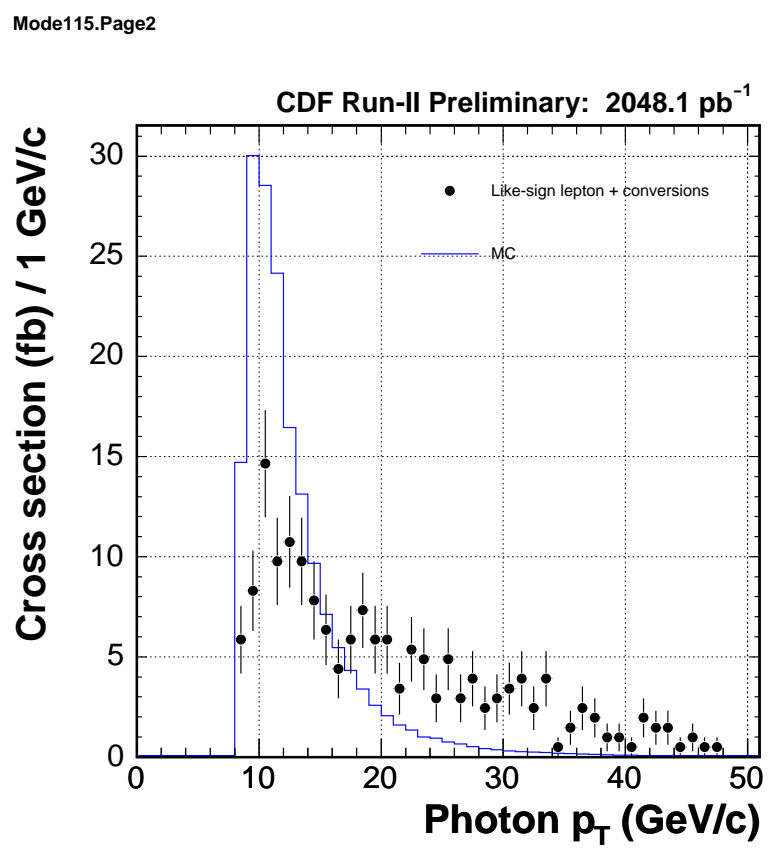

Figure 4.11: Comparison of photon- $p_{T}$ spectra between like-sign lepton + conversion events and Monte Carlo tuned by conversions in inclusive electron data.

due to statistics. From these, we believe that the systematic uncertainty is about $1 \%$ (absolute) at most, and we quote

$$
\varepsilon_{\text {pla }}=0.926 \pm 0.002 \text { (stat) } \pm 0.010 \text { (syst) } .
$$

\subsubsection{Overall Efficiency and Residual-Conversion Ratio}

We calculate the overall conversion-detection efficiency, $\varepsilon_{\text {con }}^{\gamma}\left(p_{T}\right)=\varepsilon_{\text {rel }}^{\gamma}\left(p_{T}\right) \cdot \varepsilon_{\text {pla }}$. The result is shown in Figure 4.20. We see that the $\varepsilon_{\text {con }}^{\gamma}\left(p_{T}\right)$ increases from $30 \%$ to $90 \%$ as the photon $p_{T}$ increases. Figure 4.21 shows the $R_{\text {res }}$ as a function of photon $p_{T}$.

Since the $\varepsilon_{\text {con }}^{\gamma}\left(p_{T}\right)$ is obtained by merely rescaling $\varepsilon_{\text {rel }}^{\gamma}\left(p_{T}\right)$, the relative uncertainty from MC statistics is the same as that of $\varepsilon_{\text {rel }}^{\gamma}\left(p_{T}\right)$. We then propagate it according to Eq. 4.1 to the uncertainty of the $R_{\text {res }}$. The other uncertainty of the $\varepsilon_{\text {rel }}^{\gamma}\left(p_{T}\right)$ is separately propagated to the uncertainty of the $R_{\text {res }}$. The systematic uncertainty originating from the plateau efficiency is obtained by a similar propagation which is straightforward. A summary of the relative uncertainties of the $R_{\text {res }}$ is given in Figure 4.22. Figure 4.23 shows the result for the case of the high- $p_{T}$ cut to be applied to conversions in the high- $p_{T}$ side of dileptons. 


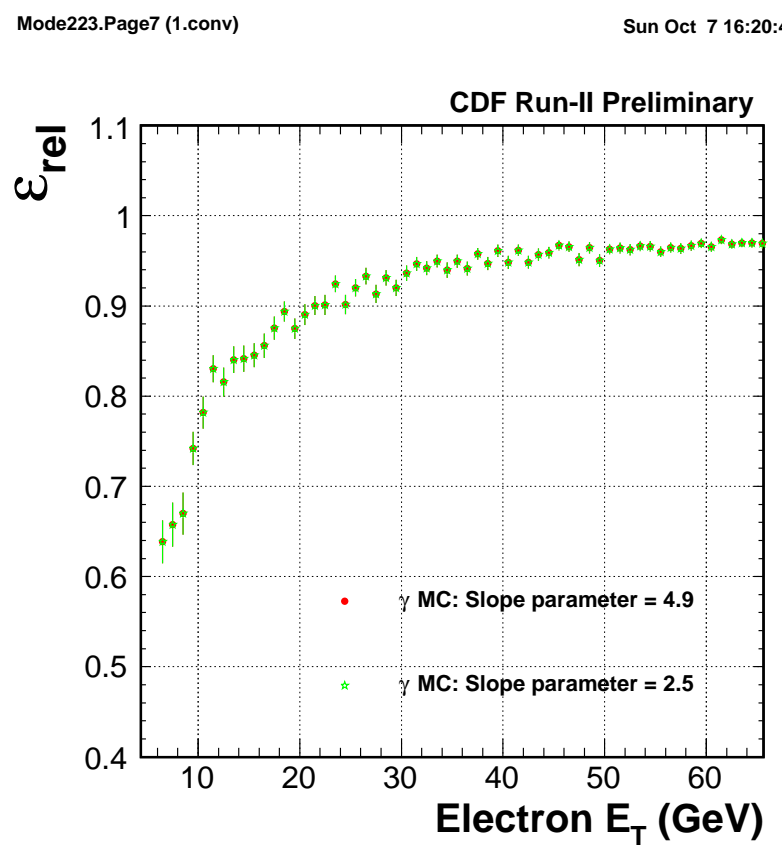

Figure 4.12: Relative conversion-detection efficiency as a function of seed-electron $E_{T}$ for two cases of slope parameters in MC tuning.

\subsubsection{Application of Residual-Conversion Ratio}

For a given conversion, we reconstruct the parent-photon $p_{T}$ from the seed-electron and its partner track, then multiply the corresponding residual conversion ratio. By summing these over all the conversions found in a sample of interest, we obtain an expectation of the total number of residual conversions. However, we usually need the kinematical information of expected residual conversions such as $p_{T}$, while we only know the $p_{T}$ of parent photons because the residual ratios is parametrized by the photon $p_{T}$. A handle of predicting kinematical information of residual conversions is the fact that they are most likely to have very low partner-track $p_{T}$, less than $1 \mathrm{GeV} / c$, as seen from the base relative efficiency shown in Figure 4.9. We, thus, approximate the partner-track $p_{T}$ of residual conversions, expected from a given conversion, to be $(0.5 \pm 0.5) \mathrm{GeV} / c$, and re-assign the difference from the original partner-track $p_{T}$ to the seed-electron $p_{T}$ :

$$
p_{T}{ }^{\text {(part) }} \rightarrow 0.5 \mathrm{GeV} / c, \quad p_{T}{ }^{(\text {seed })} \rightarrow\left(p_{T}{ }^{(\text {seed })}+p_{T}{ }^{(\text {part })}-0.5\right) \mathrm{GeV} / c .
$$

By this way, we can predict the kinematical information of residual conversions with an uncertainty of $\Delta p_{T} \simeq 0.5 \mathrm{GeV} / c$. For the conversions that already have partner-track $p_{T}$ less than $1 \mathrm{GeV} / c$, we do not perform this $p_{T}$ re-assignment.

Yet we have a problem due to the fact that the plateau region is not fully efficient. It means that conversions with partner-track $p_{T}$ of large values can still become residual 


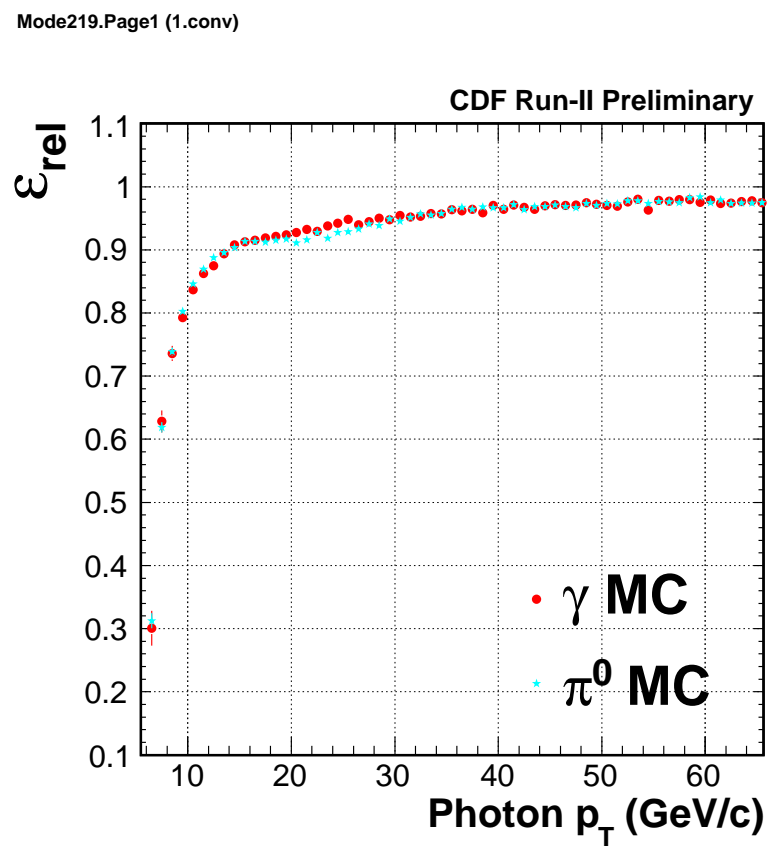

Figure 4.13: Relative conversion-detection efficiency as a function of photon $p_{T}$. The uncertainties are statistical only.

conversions at small probabilities. Performing the above $p_{T}$ re-assignment all the time is, thus, not really accurate, but using the original $p_{T}$ is also a valid thing to do reflecting the overall inefficiency of conversion detection. Let us call residual conversions with the partner-track $p_{T}$ less than $1 \mathrm{GeV} / c$ "proper" and those with $p_{T}$ larger than 1 $\mathrm{GeV} / c$ "non-proper". More precise predictions are then given by splitting residual conversions into the proper and non-proper part. The fraction of proper residualconversions for each photon $p_{T}$ bin, the "splitting" factor, can be constructed from the $\mathrm{MC}$ by weighting the generator-level spectrum of partner-track $p_{T}$ with $1-\varepsilon_{\text {abs }}\left(p_{T}\right)$, where the $\varepsilon_{\mathrm{abs}}\left(p_{T}\right)$ is the conversion detection efficiency as a function of parter-track $p_{T}$ as given in (??). The result is shown in Figure 4.24. We form a function through fitting to the plot for the $\gamma \mathrm{MC}$, which is given by

$$
\min \left(1, f_{L}\right), \quad f_{L} \equiv(2.6 \pm 0.1) \times p_{T}^{-(0.501 \pm 0.008)},
$$

where the $L$ reminds us that the parametrization corresponds to the low- $p_{T}$ cut. A similar fraction for the case of high- $p_{T}$ cut is given in Figure 4.25. The parametrized function is

$$
\min \left(1, f_{H}\right), \quad f_{H} \equiv(5.0 \pm 0.1) \times p_{T}^{-(0.663 \pm 0.009)} .
$$

The procedure for predicting residual conversions now includes one more step after obtaining the residual ratio $w$ : we further get a splitting factor $f$ using the functions above. Then the weight corresponding to the kinematics given by the $p_{T}$ re-assignment 


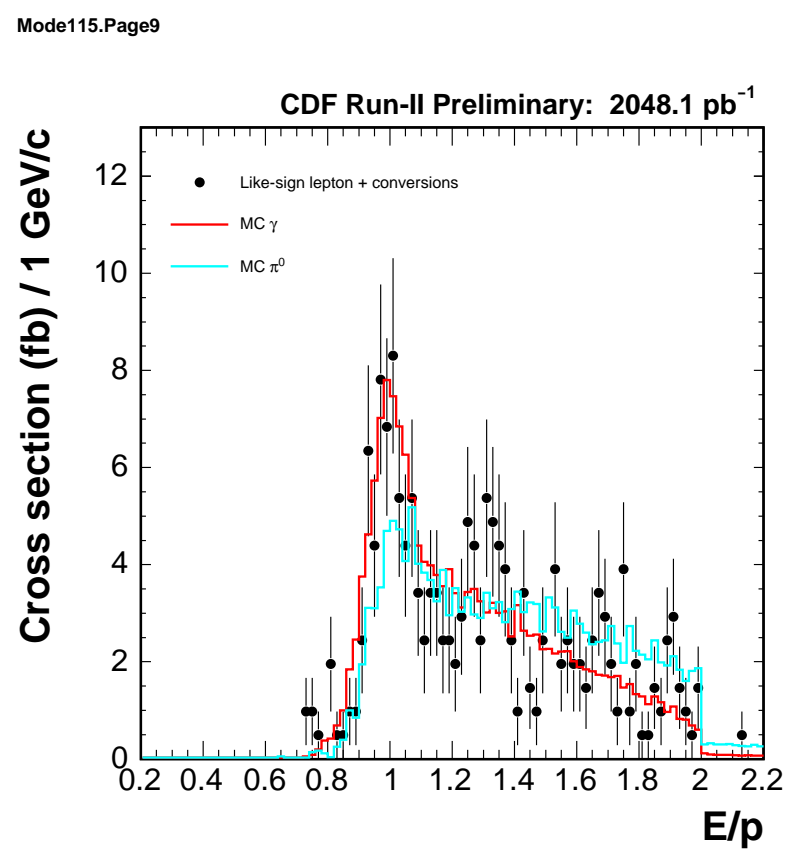

Figure 4.14: Comparison of $E / p$ distributions for conversions in like-sign lepton+conversion events, the $\gamma$ Monte Carlo, and the $\pi^{0}$ Monte Carlo. The $p_{T}$-slope parameters of the Monte Carlo data are tuned by the like-sign lepton+conversion events. The Monte Carlo distributions are normalized to the data. The uncertainties of the real data are statistical only.

is $w f$, while the weight $w(1-f)$ is applied when the original seed-electron $p_{T}$ is used as the $p_{T}$ of predicted residual conversion.

Finally, we consider another detail regarding the isolation cut. We impose a calorimeter isolation cut in the actual LS analysis. The residual conversions, therefore, must pass this cut too. But our conversion studies so far are carried out without applying the isolation cut because the MC samples of fake-events are not sufficient to simulate such information. What we need here is to introduce effects of the isolation cut specific to residual conversions that are not common to identified conversions. As we expect, residual conversions tend to pass the isolation cut more than identified conversions because the partner-track activity is less harmful. Our approach to introduce such effects is to subtract partner-track $p_{T}$ from the original isolation value, then apply the nominal isolation cut. This subtraction is coordinated with the prediction of kinematic information for expected residual conversions just described in the previous paragraphs, i.e. when the partner-track $p_{T}$ is assumed to be $0.5 \mathrm{GeV} / c$, the difference from the original value is subtracted from the isolation, and when the residual conversion is considered to be non-proper, we do not do anything to the isolation but just apply the cut as usual. Further we require the following conditions to trigger the subtraction in order to pick up only the case where the original isolation value is certainly 
Mode219.Page2 (1.conv)

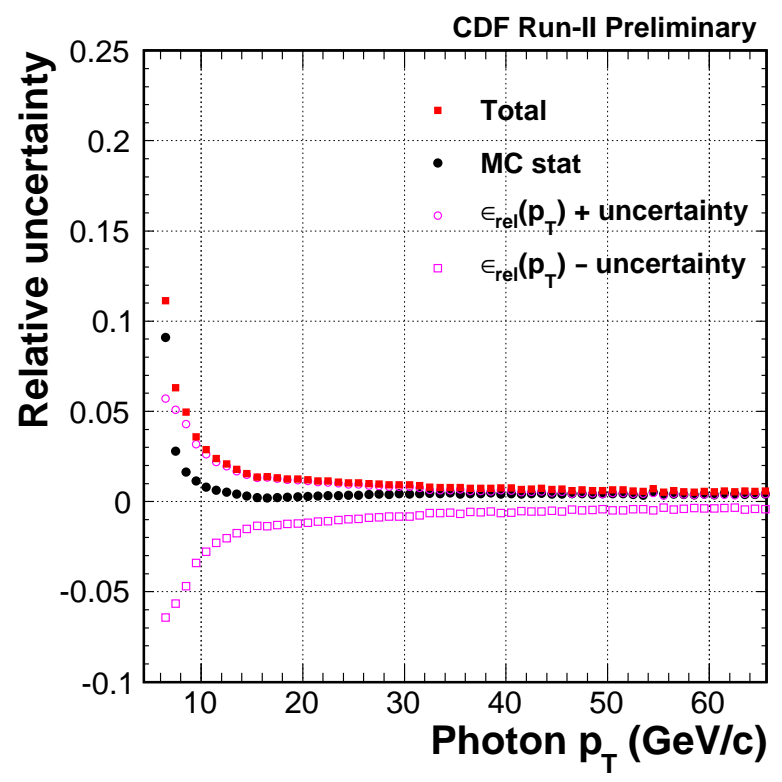

Figure 4.15: Relative uncertainty, shown for different sources, of the relative conversiondetection efficiency as a function of photon $p_{T}$.

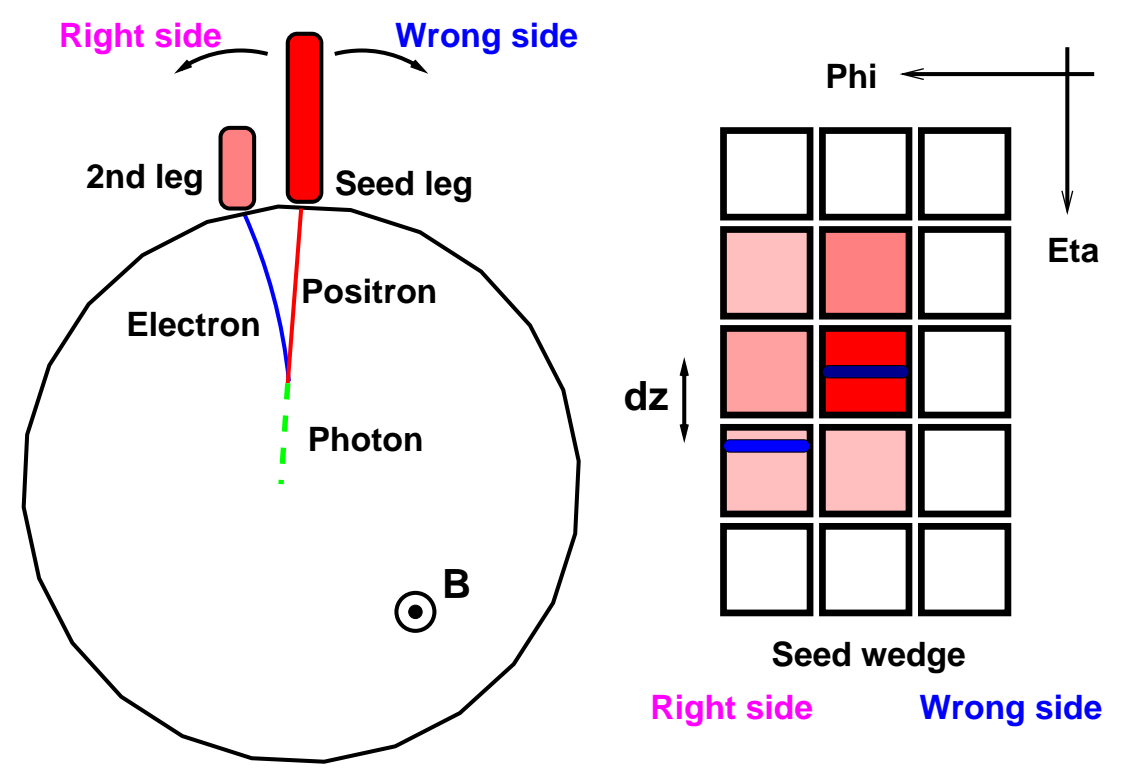

Figure 4.16: Illustration of the conversion tagging by means of CES strip information.

affected by the partner track: 


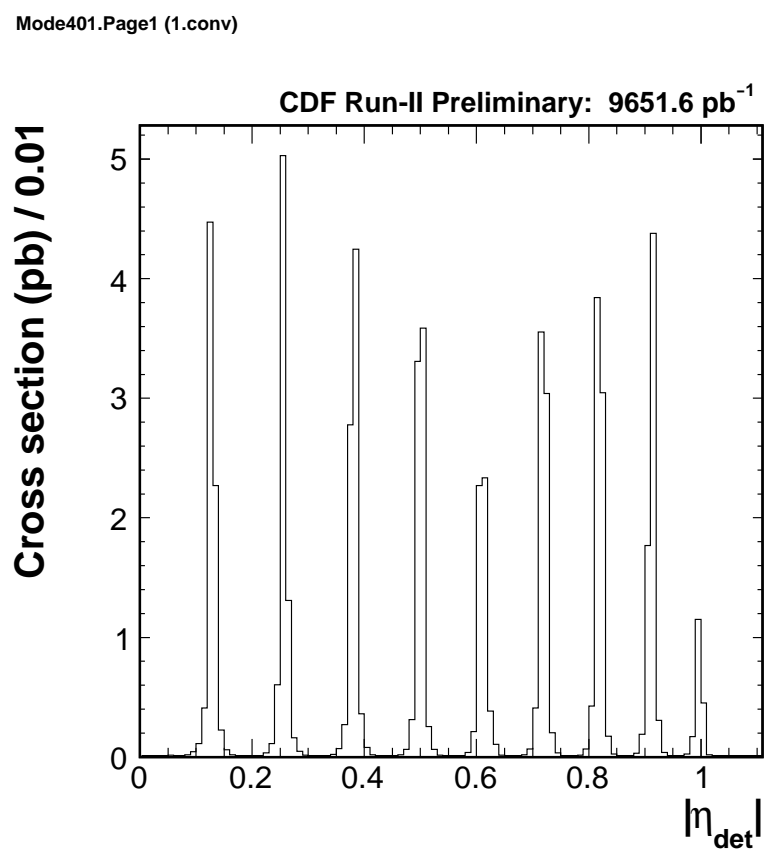

Figure 4.17: Distribution of absolute detector $\eta$ for conversion electrons with the partner track hitting the tower with an $\eta$-index different from the electron.

- the partner track is pointing a different wedge from the one of the seed electron;

- the partner track is within the isolation cone radius of 0.4 ;

- the partner track is passing through the local $x$ coordinate at the CES plane in the region $|x|<21 \mathrm{~cm}$ to avoid $\phi$ cracks.

The contribution of partner-track $p_{T}$ to the isolation is checked by looking at the ratio between the two (just like $E / p$ for electron clusters but for the isolation cone in this case) and shown in Figure 4.26. We confirm a clear peak around unity in the plot. The left side of the peak can be considered to show detector-resolution effects, and the tail in the right side can be attributed to the effects from activities around the conversions, i.e. what the isolation is meant to measure. We argue that this scheme would be capable of respecting sample dependences of the isolation, if any, because what we are trying to subtract is the activity only from the conversion partner-track, while underlying events are intact. 

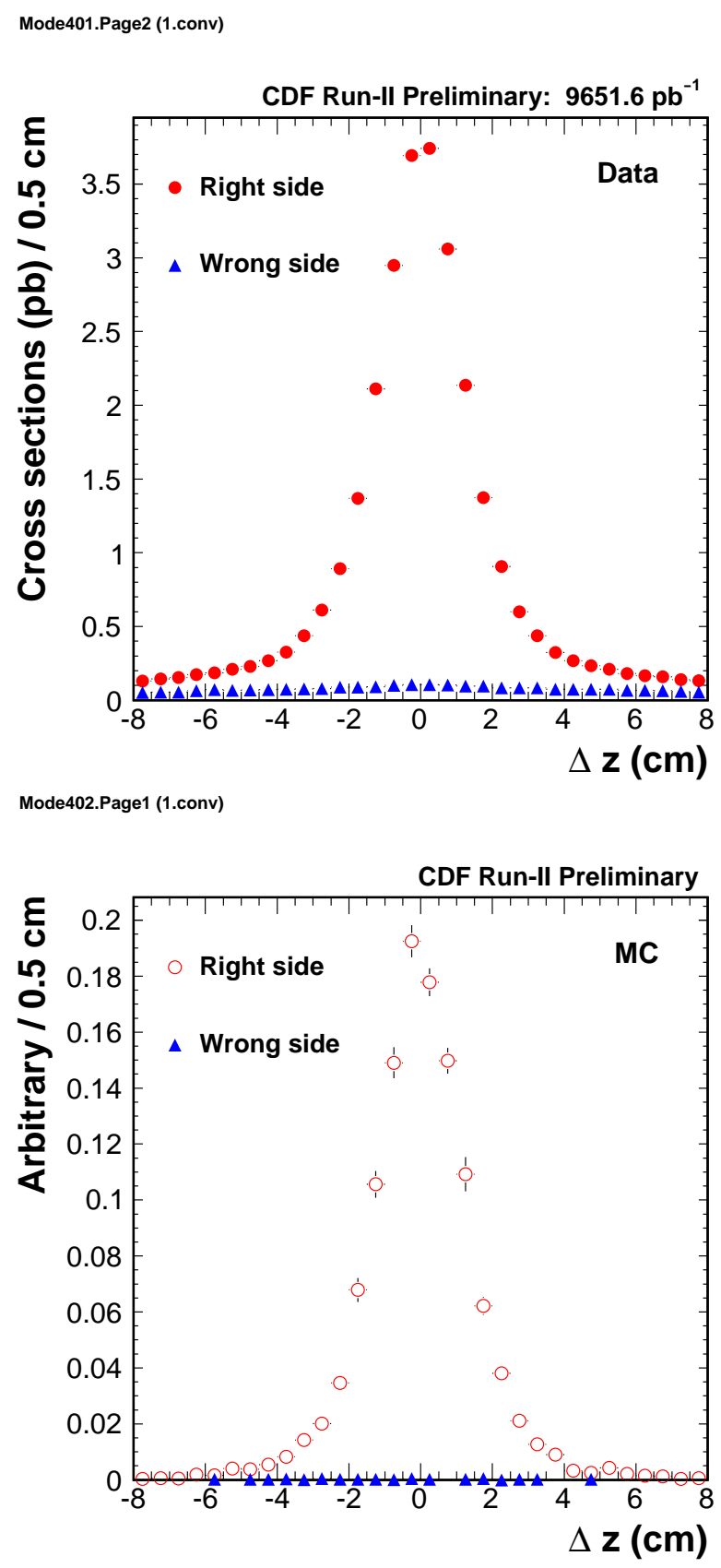

Figure 4.18: Distribution of $z$ position difference on the CES plane between the seed electron and the highest-energy CES cluster in the nearest three towers of each $\phi$ side (right side and wrong side). 
Mode401.Page5 (1.conv)
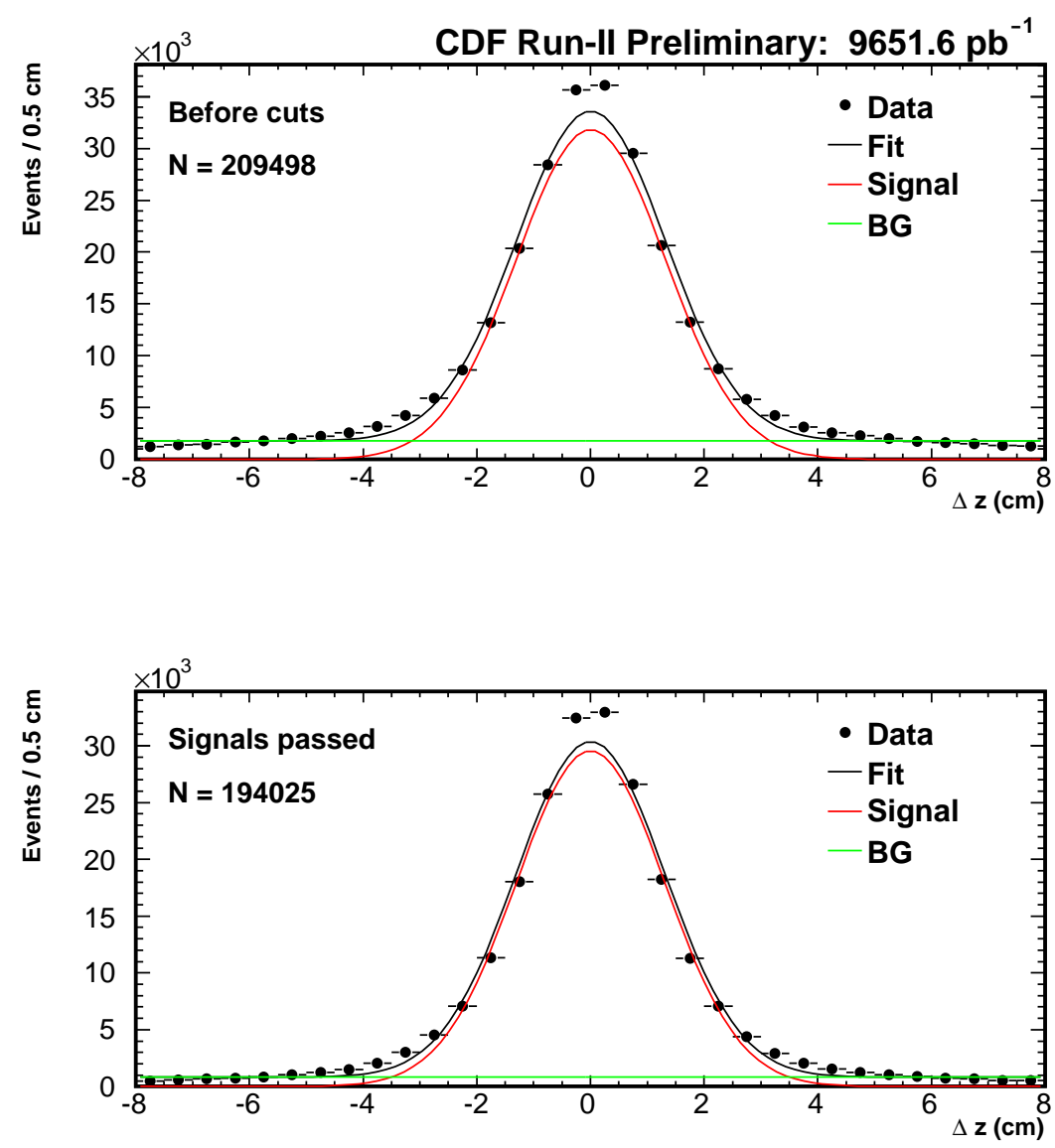

Figure 4.19: Distribution of $z$ position difference before the conversion tagging cut (top) and after (bottom). 
Mode401.Page207 (1.conv)

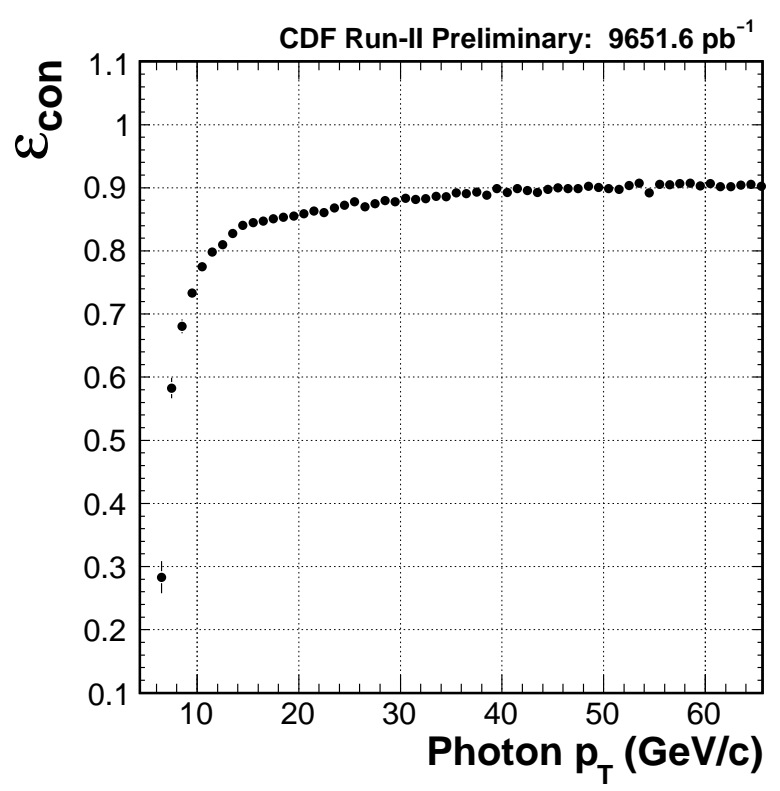

Figure 4.20: Conversion-detection efficiency as a function of photon $p_{T}$. The uncertainties are statistical only.

Mode401.Page209 (1.conv)

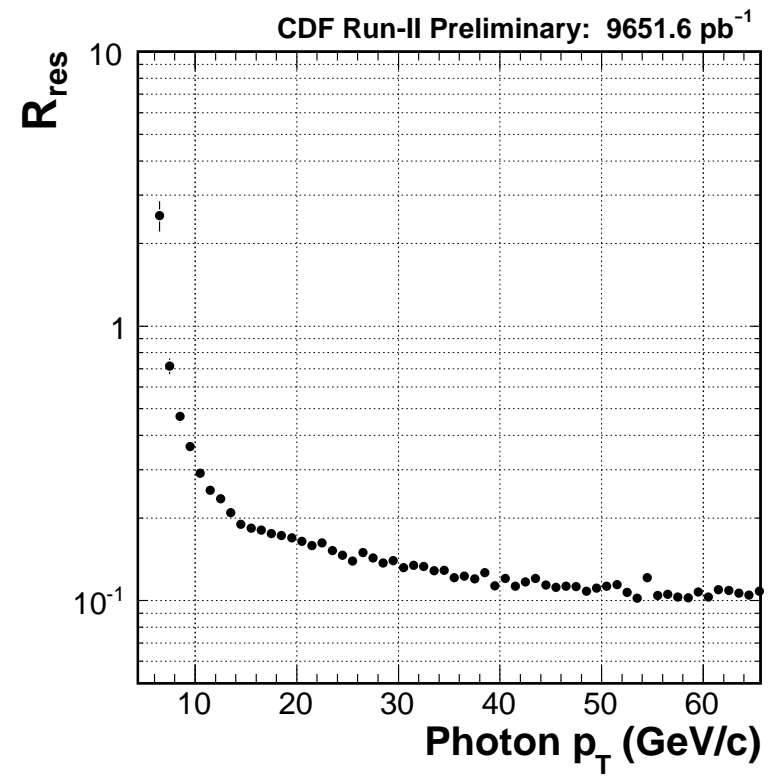

Figure 4.21: Residual ratio as a function of photon $p_{T}$. The uncertainties are statistical only. 
Mode401.Page211 (1.conv)

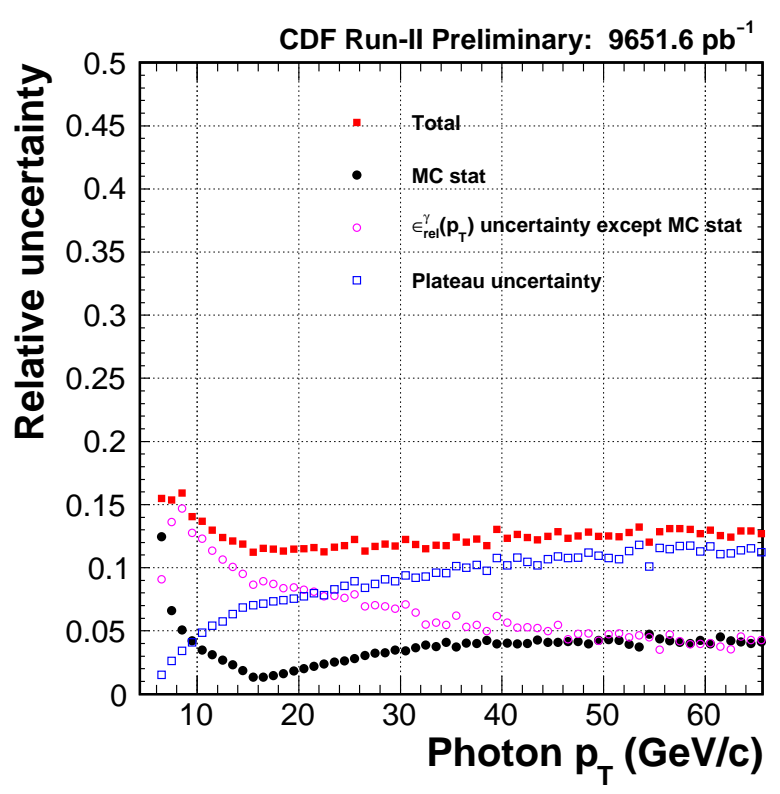

Figure 4.22: Relative uncertainty, shown for different sources, of the residual ratio as a function of photon $p_{T}$. 
Mode401.Page210 (1.conv)

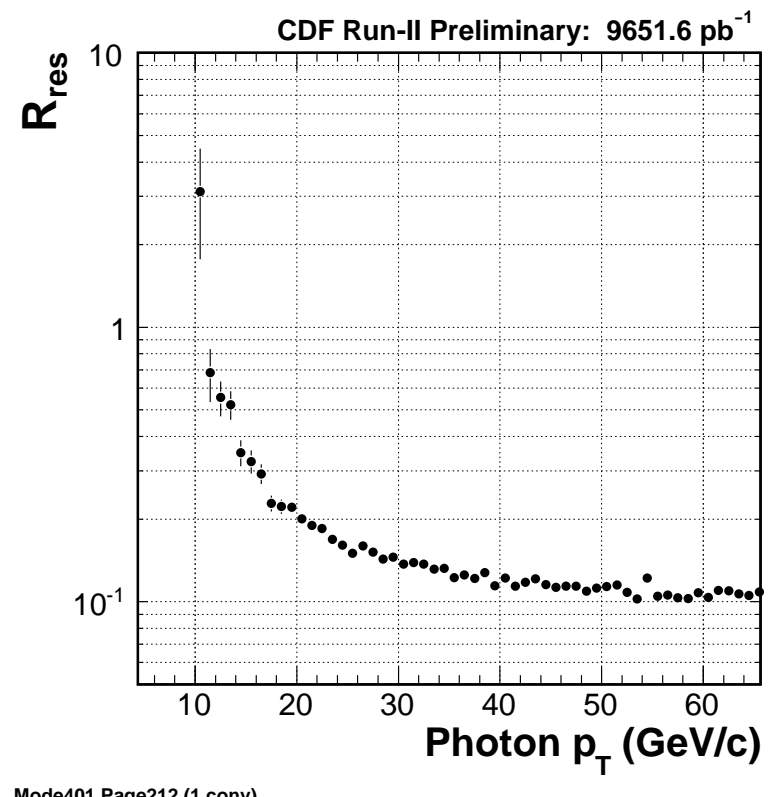

Mode401.Page212 (1.conv)

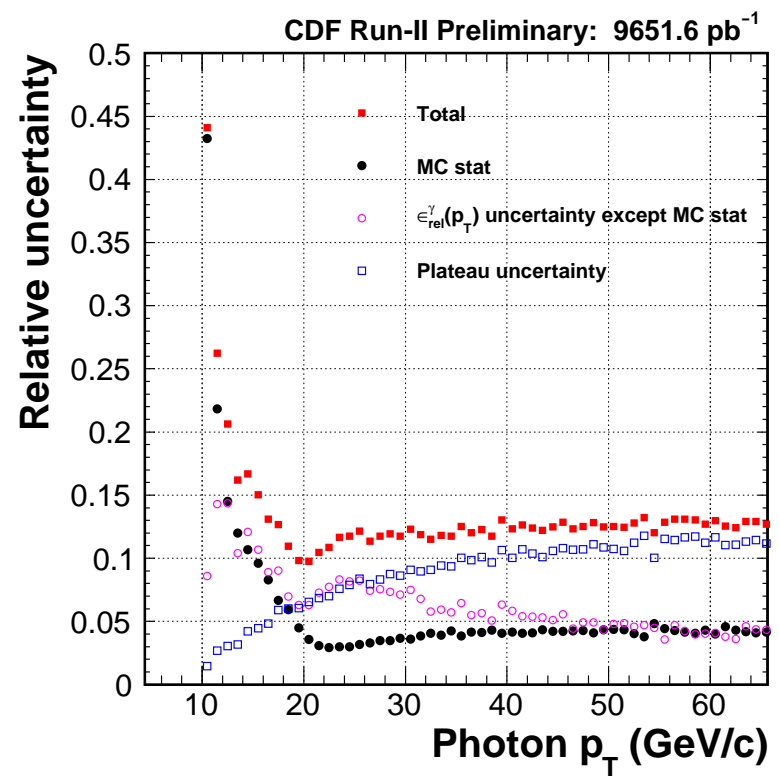

Figure 4.23: Residual ratio (top) and its relative uncertainties (bottom) as a function of photon $p_{T}$ for the case of our high- $p_{T}$ cut, $E_{T}>20 \mathrm{GeV}$ and $p_{T}>10 \mathrm{GeV} / c$. The uncertainties in the top plot are statistical only. 


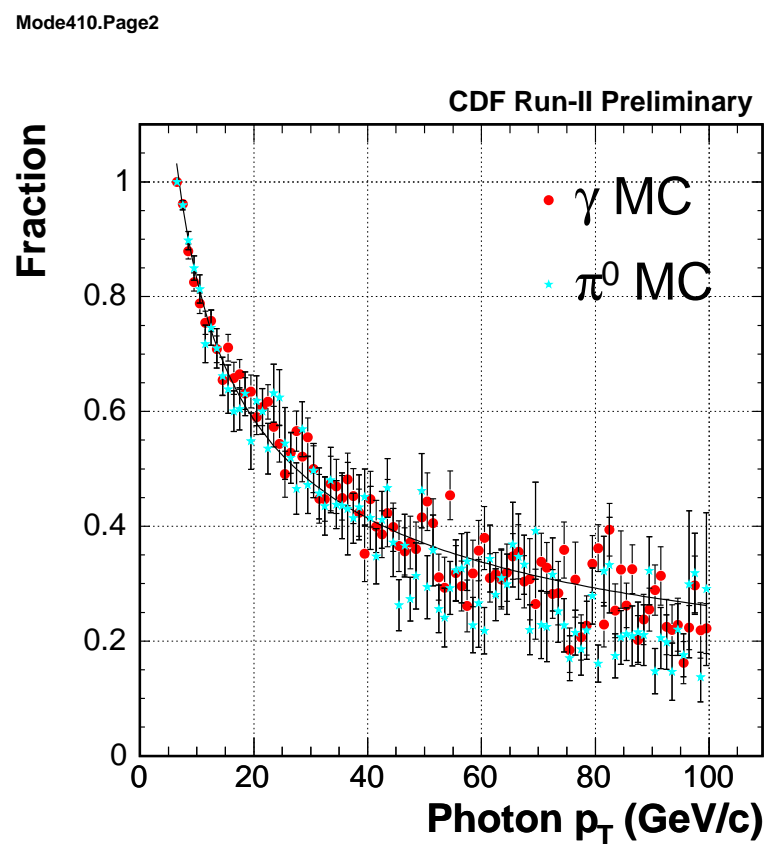

Figure 4.24: Fraction of residual conversions with partner-track $p_{T}$ less than $1 \mathrm{GeV} / c$ as a function of photon $p_{T}$ for the case of low lepton- $p_{T}$ cut.

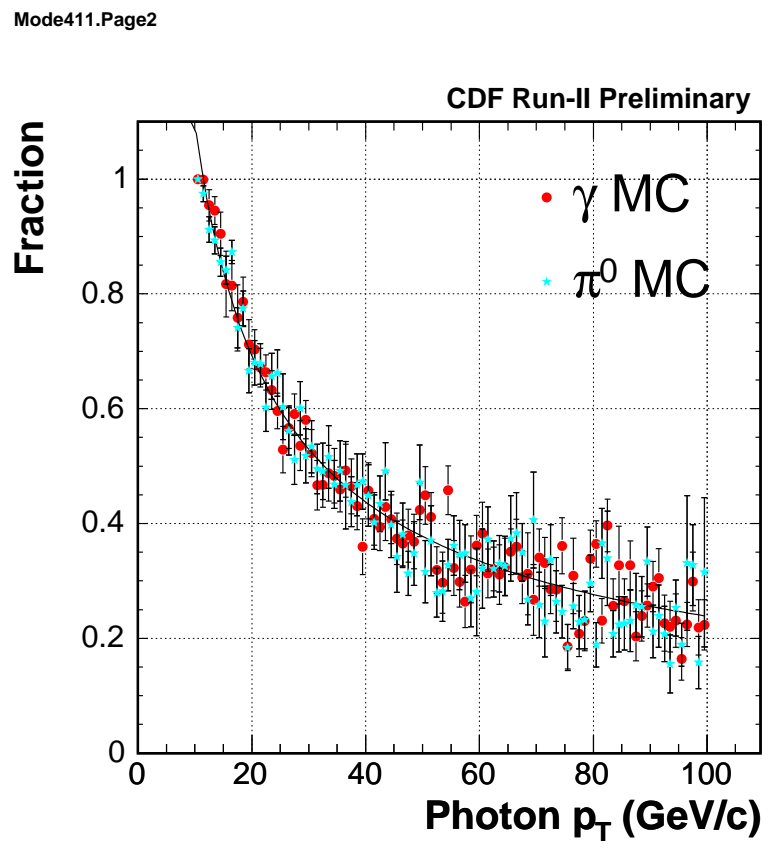

Figure 4.25: Fraction of residual conversions with partner-track $p_{T}$ less than $1 \mathrm{GeV} / c$ as a function of photon $p_{T}$ for the case of high lepton- $p_{T}$ cut. 
Mode128.Page3

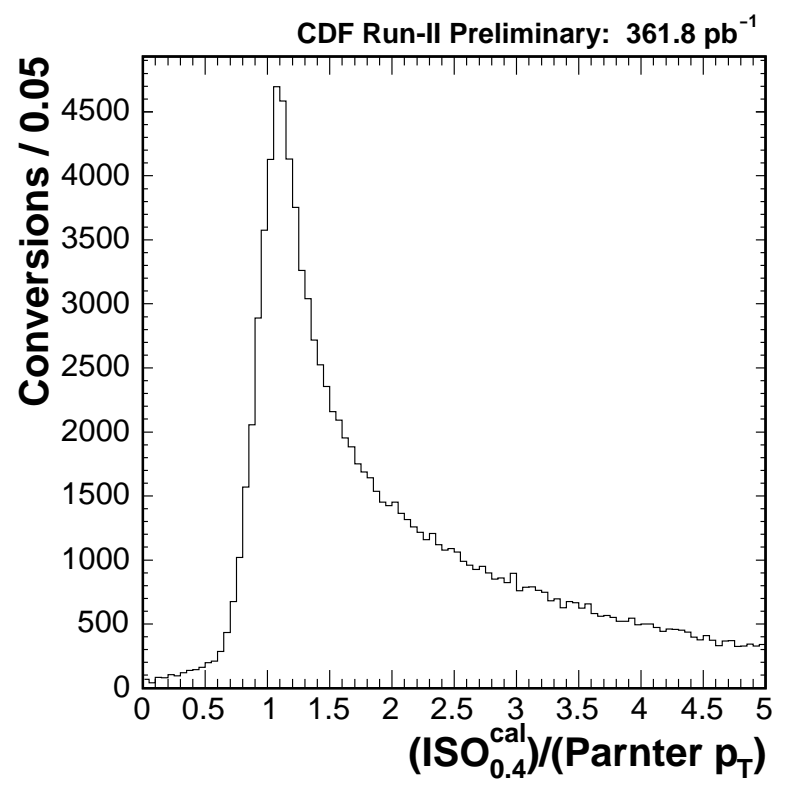

Figure 4.26: Ratio between the calorimeter isolation and the partner-track $p_{T}$ for conversions. 


\subsection{Fake Leptons}

Fake leptons are one of major backgrounds in the like-sign (LS) dilepton events. They are estimated by weighting (lepton + isolated track) events with the expected fakelepton yield for a given isolated track, called fake-lepton rates. It was studied [58] that the simple isolated-tracks in the OS combination were significantly contaminated by real leptons from Drell-Yan processes, which leads to overestimations of fake-lepton backgrounds. To avoid this problem and to establish a consistent scheme which can be applied to both the OS and LS cases, we choose isolated tracks that deposits certain energies in the electromagnetic (EM) and hadron (HA) calorimeters in the way such that they are not likely to be induced by real leptons.

\subsubsection{Fake-lepton Backgrounds}

The lepton plus fake-lepton backgrounds arise typically from a single lepton event such as $W \rightarrow \ell \nu$. This type of backgrounds consists of one trigger lepton and one fake lepton. The components of the "fake lepton" are

- Fake leptons

1. Interactive $\pi^{ \pm} \rightarrow$ fake electrons,

2. Overlap of $\pi^{0}$ and a track $\rightarrow$ fake electrons,

3. Punch-through hadrons $\rightarrow$ fake muons,

- Non-prompt leptons

1. Residual photon conversions $\rightarrow$ electrons

2. Decay-in-flight muons from $\pi^{ \pm}$and $K^{ \pm} \rightarrow$ muons,

3. (Semi-)Leptonic decay of heavy-flavor hadrons $\rightarrow$ leptons.

As noted here, we use "fake leptons" as a generic word to mean both the literal fake leptons and non-prompt leptons. Most of the components are considered to be non-isolated and quite common in generic QCD events, while the residual photon-conversions are not necessarily QCD specific, and they are separately estimated from identified conversions with a similar philosophy as the fake-lepton rates. Details of the estimation of residual photon-conversion backgrounds are discussed in $\S 4.1$. The contribution from residual conversions are subtracted from fake-electron rates in this study.

We define the fake rate $R_{\text {fake }}$ as a rate of fake leptons relative to isolated tracks with certain energy depositions especially in the hadron calorimeters, which we call "hadronic" tracks:

$$
R_{\text {fake }}=\frac{\text { lepton objects passing nominal selections and considered to be fakes }}{\text { isolated tracks with required calorimeter energy depositions }} .
$$


It is noted that we define the fake rate as per isolated track, not per jet. The event preselection and the isolated hadronic-track selection criteria are listed in Table 4.2. The kinematical cut is $p_{T}>6 \mathrm{GeV} / c$ and we require the same track-quality cuts as shown in Table 3.6. The hadronic-track selection imposes cuts on the energy depositions in the calorimeter towers that a given track is pointing. The first cut in the table is thought to mainly reject real electrons and the other cuts are meant to reject real muons. On the other hand, in the numerator, we apply the nominal lepton selection cuts to find fake-lepton objects.

We evaluate $R_{\text {fake }}$ using inclusive jet samples. The data we use are the Jet20, Jet50, Jet70, and Jet100 generic jet samples from the 0d through the 0p datasets. The "jet samples" mean different types of jet data collected by different triggers. First, we require that the hadronic tracks and fake-lepton objects be both separated from the trigger jet in the $\eta$ - $\phi$ space by $R>1.0$ to remove trigger biases as possible.

Next, to remove real-leptons in the numerator, we impose a $Z$ veto. The $Z$ events defined by the following are rejected:

- electrons with $E_{T}>20 \mathrm{GeV}$ or muons with $p_{T}>20 \mathrm{GeV} / c$ passing our lepton selection, and

- invariant mass with the 2nd-leg objects is in the mass window of $81-101 \mathrm{GeV} / c$.

The definition of the 2nd-leg objects for the $Z$ veto is given in Table 3.8. Note that the EM objects for the 2nd-leg are not necessarily in the central region.

In the previous analysis [58], we also used $W$ veto defined as

- electrons with $E_{T}>20 \mathrm{GeV}$ or muons with $p_{T}>20 \mathrm{GeV} / c$ passing lepton selection shown in Table 3.6,

- $\mathbb{E}_{T}>30 \mathrm{GeV}$ or transverse mass $>40 \mathrm{GeV} / c^{2}$,

where $\mathbb{E}_{T}$ is corrected for muons passing our muon selection with the $p_{T}>6 \mathrm{GeV} / c$ cut. However, such the veto causes some bias in $p_{T}$ distribution, shown in Figure 4.27. Instead of $W$ veto algorithm, we estimate these components using $W$ and $Z$ MC samples, and subtract them from the numerator in jet samples. The $Z$ contributions is small compared to the $W$ events due to $Z$ veto, but it still remains. Normalizations of MC samples are estimated using distributions of transverse mass to take the complicated trigger systems into account. Figure 4.28 shows the transverse mass distribution in jet samples. In the plot, we fit the $\mathrm{MC}$ to the data using the function

$$
p_{0} \cdot(\mathrm{MC} \text { histogram })+\exp \left(p_{1} x+p_{2}\right)
$$

where the component of exponential represents the fake lepton distribusion we have assumed, and $p_{0}, p_{1}, p_{2}$ are the fitting parameters. $p_{0}$ determines a normalization of MC samples. Using the normalization, we rescale MC samples and subtract the $W$ and $Z$ components from the jet samples. Figure 4.29 and Figure 4.30 shows $p_{T}$ distributions of fake leptons. Using these distribusions, we estimate fake rates of leptons. 
Fake rates parametrized by $p_{T}$ is commonly used in CDF. The rates, however, depend on other variables. To take the dependencies, we choose six variables: fake-lepton transverse momentum $\left(p_{T}\right)$, pseudorapidity $(\eta)$, isolation $\left(\mathrm{ISO}_{0.4}^{\mathrm{cal}}\right)$, impact parameter $\left(d_{0}\right)$, offset angle of a calorimeter wedge $\left(\phi_{w}\right)$, and difference of azimuthal angle between fake lepton and missing $E_{T}\left(\Delta \phi\left(\ell, E_{T}\right)\right)$. Then, we parametrize fake rates $R_{\text {fake }}$ as

$$
R_{\mathrm{fake}}=\frac{n}{d} \cdot r\left(p_{T}\right) \cdot r(\eta) \cdot f\left(\mathrm{ISO}_{\text {cal }}^{0.4}\right) \cdot f\left(d_{0}\right) \cdot f\left(\phi_{w}\right) \cdot f\left(\Delta \phi\left(\ell, E_{T}\right)\right),
$$

where

- $n$ : number of fake leptons,

- $d$ : number of hadronic tracks,

- $r()$ : Normalized binned rate,

- $f()$ : Normalized rate fitting by cubic function $\left(a x^{3}+b x^{2}+c x+d\right)$.

The absolute fake rates are determined by $d / n$ in eq. (4.16). For electrons and muons, the rates are $0.019 \pm 0.001$ and $0.078 \pm 0.001$, respectively. These normalized rates are shown in Figure 4.31 and Figure 4.32.

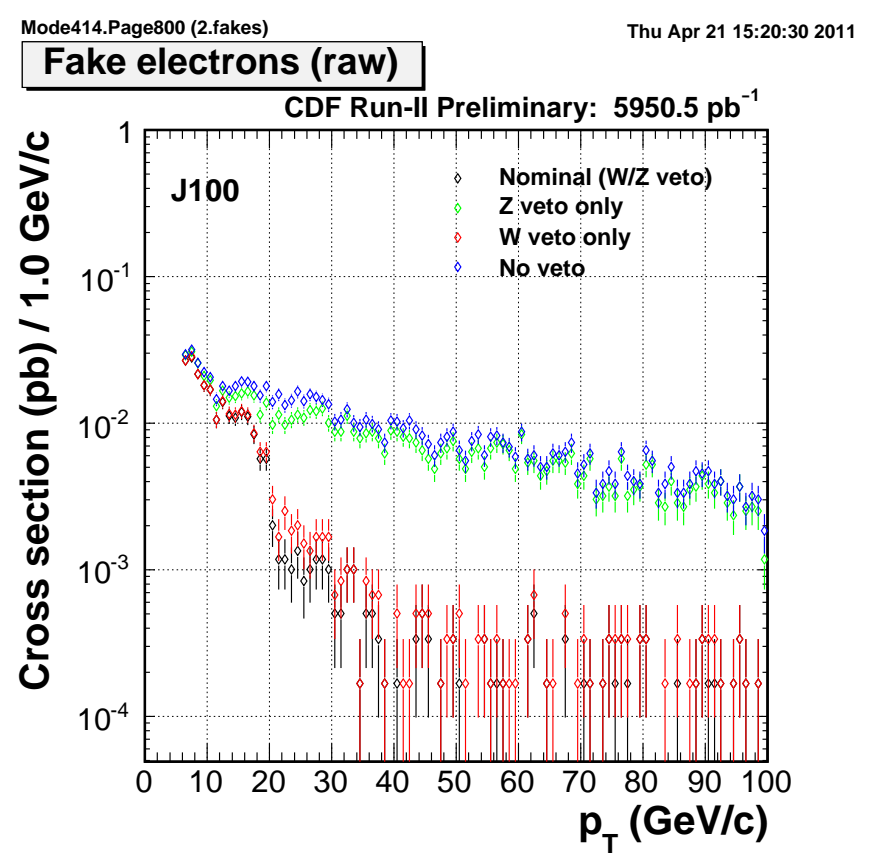

Figure 4.27: Bias due to $\mathrm{W}$ veto in jet samples. 


\begin{tabular}{c}
\hline \hline$\frac{\text { Event pre-selection }}{\left|z_{\text {pv }}\right|<60 \mathrm{~cm}}$ \\
Cosmic-ray veto \\
Geometrical and kinematical cuts \\
CDF default tracks in the central region \\
$p_{T}>6 \mathrm{GeV} / \mathrm{c}$ \\
$\underline{\text { Track quality cuts }}$ \\
and stereo $\geq 3(\geq 7$ hits $)$ \\
$\left|z_{0}-z_{\text {pv }}\right|<2 \mathrm{~cm}$ \\
Silicon hits $\geq 3$ \\
$\left|d_{0}\right|<0.02 \mathrm{~cm}$ \\
Isolation cut \\
ISO ${ }_{0.4}^{\text {cal }}<2 \mathrm{GeV}$ \\
Hadronic-track selection \\
HA $/$ EM $>0.2$ \\
EM $>1 \mathrm{GeV}$ \\
HA $>4 \mathrm{GeV}$ \\
\hline \hline
\end{tabular}

Table 4.2: Event pre-selection and the denominator track selection.
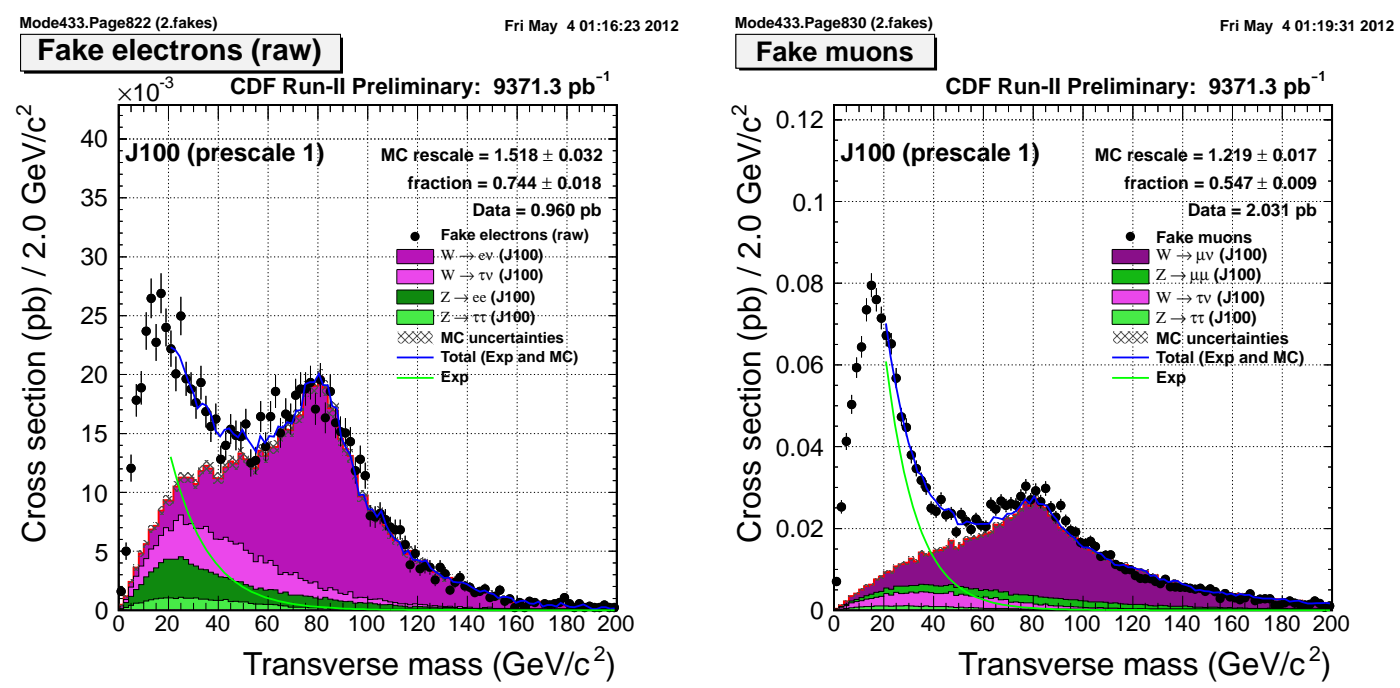

Figure 4.28: Transverse mass distribution. 

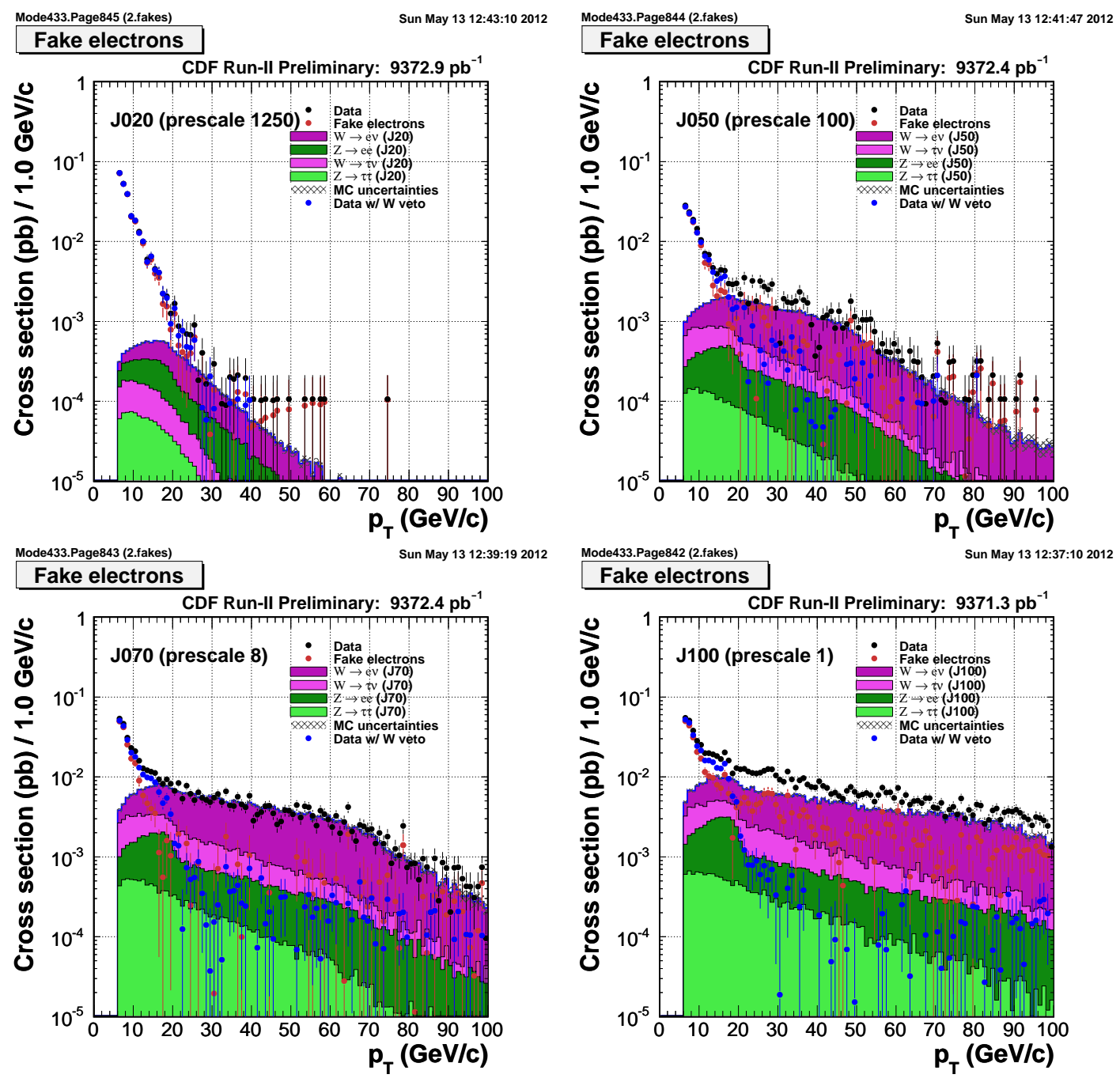

Figure 4.29: $p_{T}$ distributions in each jet sample. 


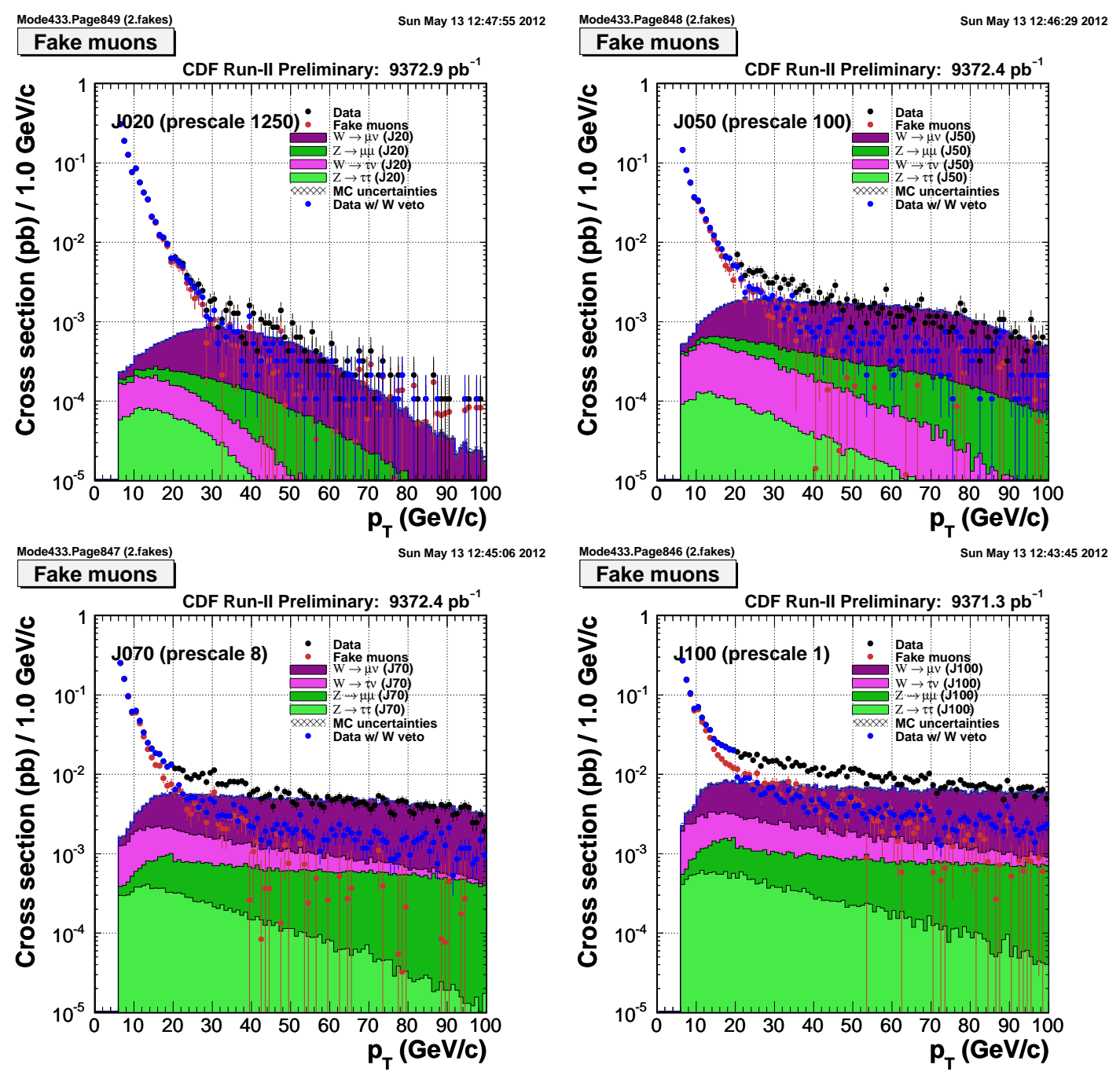

Figure 4.30: $p_{T}$ distributions in each jet sample. 

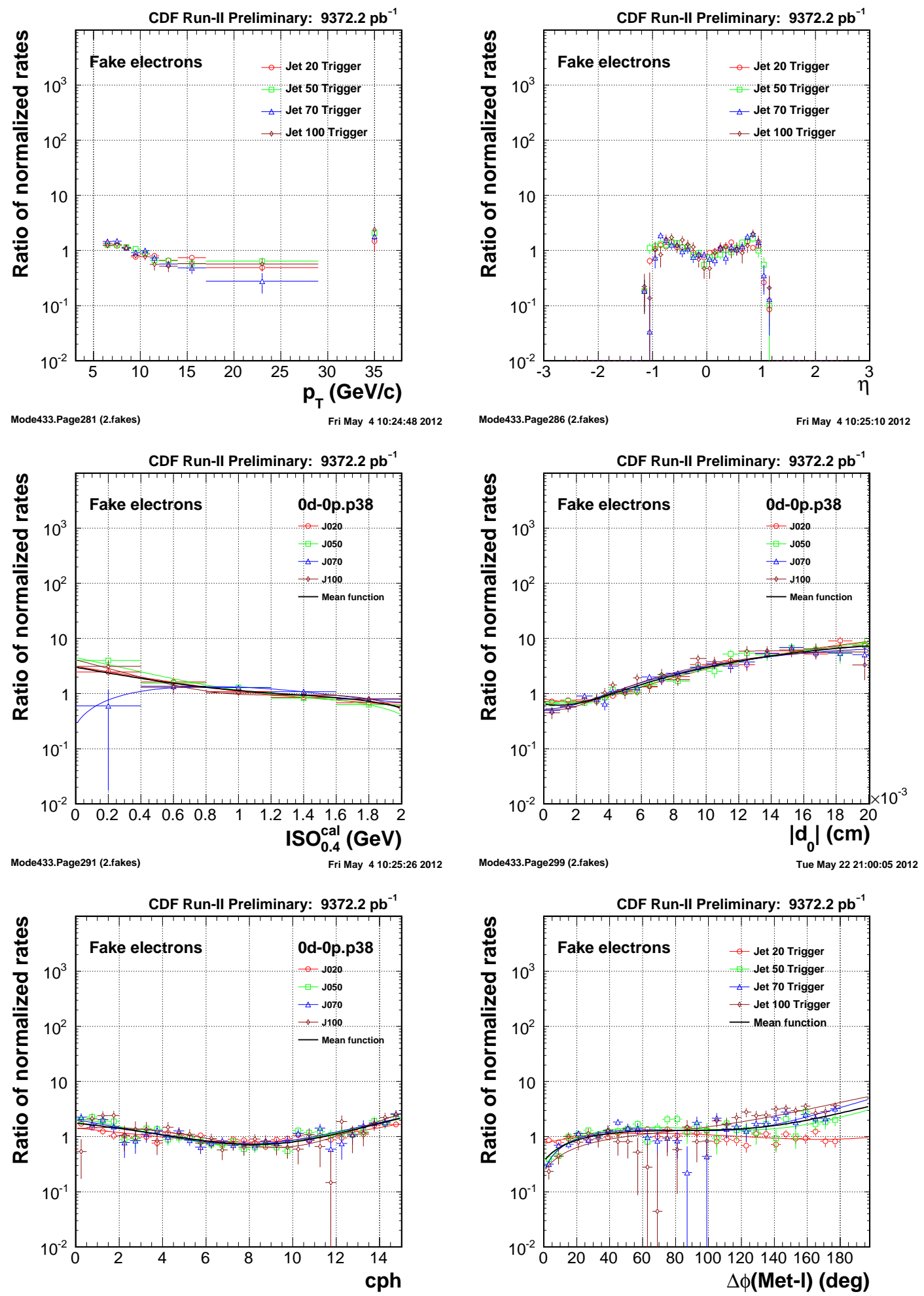

Figure 4.31: Fake rates of electrons. The variables are lepton $p_{T}$, pseudorapidity $\eta$, isolation, impact parameter $d_{0}$, the offset angle of one calorimeter (cph), and the difference angle between lepton and missing $E_{T}$. 

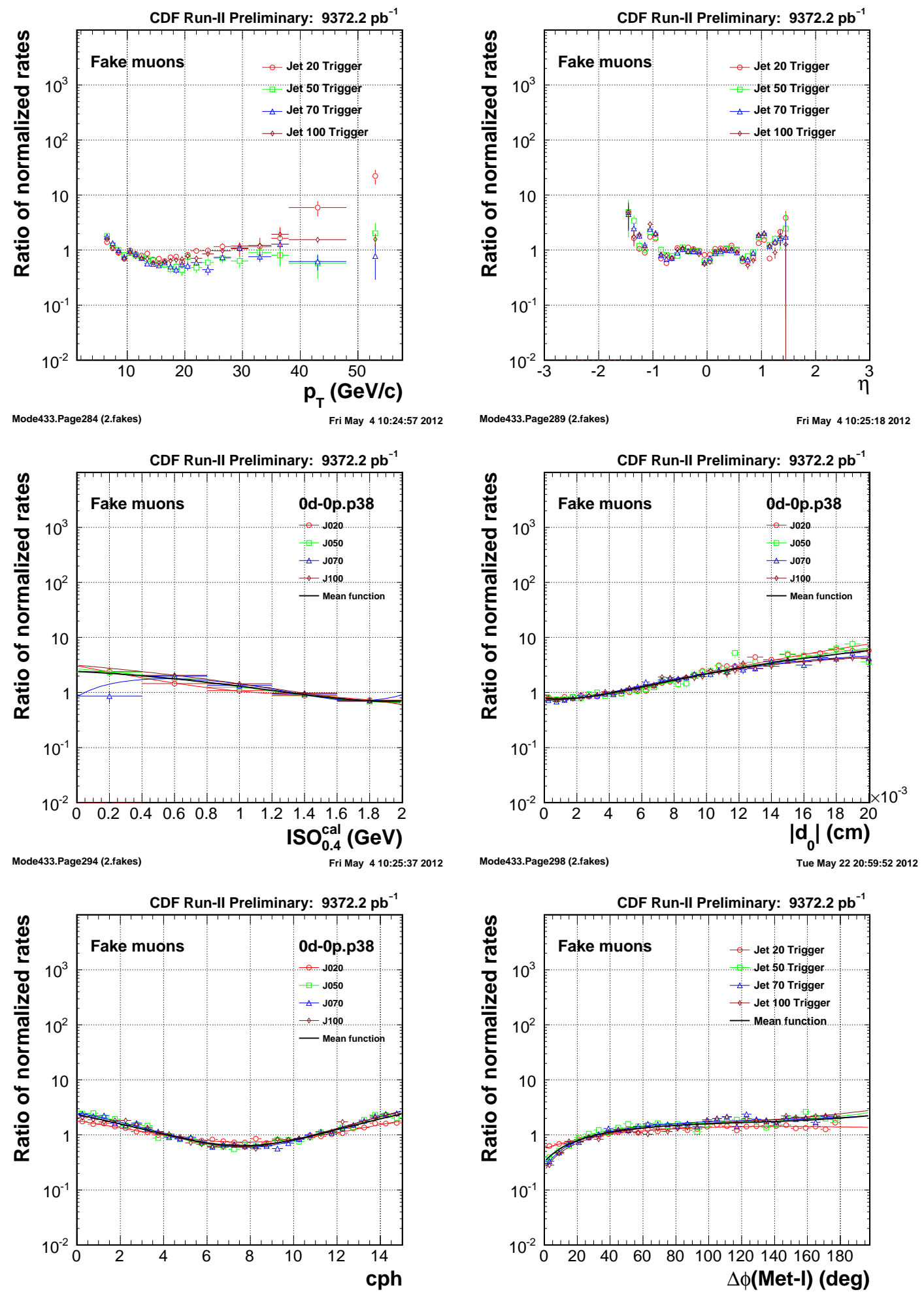

Figure 4.32: Fake rates of muons. The variables are lepton $p_{T}$, pseudorapidity $\eta$, isolation, impact parameter $d_{0}$, the offset angle of one calorimeter (cph), and the difference angle between lepton and missing $E_{T}$. 


\subsection{Physics Backgrounds}

Physics backgrounds can be classified into reducible and irreducible backgrounds:

- Reducible backgrounds

1. $Z / \gamma^{*} \rightarrow \ell^{ \pm} \ell^{\mp}$,

2. $W+$ (heavy-flavor hadrons) $\rightarrow \ell \ell+\mathrm{X}$,

3. $t \bar{t} \rightarrow\left(W^{+} b\right)\left(W^{-} b\right) \rightarrow \ell \ell+X$,

4. $W^{ \pm} W^{\mp} \rightarrow\left(\ell^{+} \nu\right)\left(\ell^{-} \nu\right)$,

- Irreducible backgrounds

1. $W Z \rightarrow\left(\ell^{ \pm} \nu\right)\left(\ell^{+} \ell^{-}\right)$,

2. $Z Z \rightarrow\left(\ell^{+} \ell^{-}\right)\left(\ell^{+} \ell^{-}\right)$.

The reducible backgrounds can be reduced by like-sign charge requirement. These background contributions mainly come from residual conversions and fake leptons. For irreducible backgrounds, we can surpress the contribution by requiring $Z$ veto to some extent.

We estimate the physics backgrounds using MC samples, which is created by PYTHIA event generator [62]. Table 4.3 lists the MC samples. In the MC samples, residual conversions and fake lepton events are explicitly removed from MC contributions by looking at the OBSP information since these contributions are estimated from the data-driven method independently described in $\S 4.1$ and $\S 4.2$.

\subsubsection{Monte Carlo Scale Factors}

After MC event generations, these events are passed into the detector simulation based on GEANT 4 [63], which allows the construction of a mathematical model of detectors: for example, showering to secondary or tertiary particles, charge deposition models of the response of the tracking detectors. The detector simulation, however, is not perfectly modeled to the real detectors. It is the scale factors to scale MC events to the expected events in the CDF data. The number of events is given by

$$
N=L \cdot \varepsilon \cdot \sigma,
$$

where $L$ is the integrated luminosity, $\varepsilon$ is the total detection efficiency, and $\sigma$ is the cross section of some process. The total detection efficiency includes several parts, and can be expressed as

$$
\varepsilon=\sum_{i} A^{(i)} \cdot \varepsilon_{\text {trig }}^{(i)} \cdot \varepsilon_{\text {pre }}^{(i)} \cdot \varepsilon_{\mathrm{ID}}^{(i)}
$$

where $i$ means dilepton types such as CEM-CEM, CMUP-CMX, and so on. $A^{(i)}$ is the geometrical and kinematical acceptances, $\varepsilon^{(i)}$ is the trigger efficiency, $\varepsilon_{\text {pre }}^{(i)}$ is the pre-selection efficiency, and $\varepsilon_{\mathrm{ID}}^{(i)}$ is the lepton identification efficiency. 


\begin{tabular}{lcccccc}
\hline \hline Process & Events & $\sigma \cdot B_{F}$ & $\begin{array}{c}K \text { factor } \\
(\mathrm{pb})\end{array}$ & $\begin{array}{c}\text { Filter- } \\
\text { efficiency }\end{array}$ & $\begin{array}{c}\text { Luminosity } \\
\left(\mathrm{fb}^{-1}\right)\end{array}$ & Notes \\
& & & & \\
\hline
\end{tabular}

Electron channels

\begin{tabular}{lcccccc}
\hline$W \rightarrow e \nu$ & 4036290 & 1960 & 1.4 & - & 1.47 & \\
$Z / \gamma^{*} \rightarrow e e$ & 1594110 & $355 \pm 3$ & 1.4 & - & 3.21 & $M>20 \mathrm{GeV} / c^{2}$ \\
\multicolumn{2}{l}{ Muon channels } & & & & & \\
\hline$W \rightarrow \mu \nu$ & 1608588 & 1960 & 1.4 & - & 0.586 & \\
$Z / \gamma^{*} \rightarrow \mu \mu$ & 6701700 & $355 \pm 3$ & 1.4 & - & 13.5 & $M>20 \mathrm{GeV} / c^{2}$ \\
\multicolumn{2}{l}{ Both channels } & & & & & \\
\hline$Z / \gamma^{*} \rightarrow \tau \tau$ & 6849882 & $238 \pm 3$ & 1.4 & - & 19.0 & $M>30 \mathrm{GeV} / c^{2}$ \\
$t \bar{t}$ & 1111652 & 7.3 & - & - & 152.3 & \\
$W W$ & 1596781 & 1.27 & - & - & $1.26 \times 10^{3}$ & \\
$W Z$ & 3598792 & 0.365 & - & 0.76 & $1.19 \times 10^{4}$ & \\
$Z Z$ & 761342 & 2.01 & - & 0.23 & $1.65 \times 10^{3}$ & $M>15 \mathrm{GeV} / c^{2}$ \\
\end{tabular}

Table 4.3: Summary of the Monte Carlo samples.

The trigger efficiency is estimated using real data, while other efficiencies are using data and MC samples.

\subsubsection{Data Samples}

As described in $\S 3.1$, the data have been collected by inclusive high- $p_{T}$ lepton triggers. The triggers we use are ELECTRON_CENTRAL_18, MUON_CMUP18, and MUON_CMX18. These trigger efficiencies are used as a part of MC scale factors.

These triggers can effectively collect electroweak events such as Drell-Yan events. The Drell-Yan events are used to estimate the pre-selection and the lepton identification efficiencies.

\subsubsection{Trigger Efficiency}

The trigger efficiencies are estimated in each period from the period 0 through 38 to take the changes of the trigger tables into account.

\section{High- $p_{T}$ Electron Trigger Efficiency}

The efficiency of the electron trigger can be divided into two parts: tracking trigger part and calorimeter trigger part. We use W_NOTRAK trigger to estimate tracking 
trigger efficiency. The W_NOTRAK trigger has the same calorimeter trigger path as the ELECTRON_CENTRAL_18 trigger path, but does not have the tracking trigger path. For the tracking trigger efficiency, we pick up $W$ candidate events which have good electrons passing our electron selections, missing $E_{T}>25 \mathrm{GeV}$, and W_NOTRAK trigger bits. These are the denominator of the efficiency. Then we require the tracking trigger at each level to estimate the efficiencies.

For the Level-1 calorimeter trigger efficiency, we use high- $p_{T}$ muon samples as unbiased samples. In muons samples, we pick up good electrons passing our electron selections (the L1 denominator), then require the event to fire the L1_EM8 trigger (the L1 numerator). The L1_EM8 trigger is the same as the Level-1 trigger of ELECTRON_CENTRAL_18 (L1_CEM8_PT8) for the calorimeter trigger part. For the Level2 calorimeter trigger efficiency, we use good lepton events which are triggered by the ELECTRON_CENTRAL_18_NO_L2 and the Level-1 trigger of ELECTRON_CENTRAL_18 (L2 denominator), then we require the Level-2 trigger of ELECTRON_CENTRAL_18 (L2 numerator). For the Level-3 caorimeter trigger efficiency measurement, we pick up good lepton events passing the ELECTRON_CENTRAL_18_NO_L2 and the Level-2 trigger of ELECTRON_CENTRAL_18 (L3 denominator), then we require the Level-3 trigger (L3 numerator).

The efficiency in each period is shown in A.1.

\section{High- $p_{T}$ Muon Trigger Efficiency}

We use $Z \rightarrow \mu \mu$ events which have the invariant mass between 81 to $101 \mathrm{GeV} / c^{2}$ and fire the MUON_CMX18 (MUON_CMUP18) trigger (the denominator objects). Then we apply the MUON_CMUP18 (MUON_CMX18) trigger (the numerator objects).

The efficiency in each period is shown in A.1.

\subsubsection{Primary-Vertex Cut Efficiency and Scale Factor}

Primary-vertex cut is to select events within the region of well-defined measurement of collisions. The region is confirmed as $\left|z_{\mathrm{pv}}\right|<60 \mathrm{~cm}$ by using cosmic ray and simulations. For both the cosmic ray track $z_{0}$ and the simulation $z_{0}$, the efficiency within $\left|z_{0}\right|<60 \mathrm{~cm}$ is flat. The acceptance of the $p \bar{p}$ beam luminous region of $\left|z_{0}\right|<60 \mathrm{~cm}$ is determined by fitting to "beam luminosity function" with $\left|z_{0}\right|<60 \mathrm{~cm}$. The beam luminosity function is the longitudinal profile of the luminous region, expressed as

$$
\frac{d L(z)}{d z}=N_{p} N_{\bar{p}} \frac{1}{\sqrt{2 \pi} \sigma_{z}} \frac{\exp \left(-z^{2} / 2 \sigma_{z}^{2}\right)}{4 \pi \sigma_{x}(z) \sigma_{y}(z)}
$$

where $z$ is the primary-vertex position, $N_{p, \bar{p}}$ is proton or antiproton beam flux, and $\sigma_{x, y, z}(z)$ is the beam width on each axis. The efficiency is calculated from the fit to $z$ of the beam profile:

$$
\varepsilon\left(\left|z_{0}\right|<60 \mathrm{~cm}\right)=\frac{\int_{-60}^{+60}[d L(x) / d z] \mathrm{d} z}{\int_{-\infty}^{+\infty}[d L(x) / d z] \mathrm{d} z}
$$


We use high- $p_{T}$ lepton trigger samples and Drell-Yan Monte Carlo samples to estimate the efficiencies and scale factors. The efficiency in each dataset is shown in A.2.

\subsubsection{Lepton Identification Efficiency and Scale Factor}

Our kinematical cut is $p_{T}>20 \mathrm{GeV} / c$ for the leading lepton and $p_{T}>6 \mathrm{GeV} / c$ for the 2nd leading lepton. Basically, we have to estimate the efficiency and scale factor from $p_{T}>6 \mathrm{GeV} / c$. We use Drell-Yan events of the data and MC samples to estimate efficiencies and scale factors. For the electrons, we estimate the efficiencies in two regions with respect to electron $E_{T}$, because the efficiencies of the electron identification depends on it. Therefore, we divide two regions at $E_{T}=30 \mathrm{GeV}$. For muons, on the other hand, the efficiencies do not show any clear dependence.

\subsubsection{OSLS Fake Ratio}

The Drell-Yan events are oppositely-charged dilepton events which are passing $\Delta \phi_{\ell_{1} \ell_{2}}>$ 2.8 (rad) cut. Such Drell-Yan events contain non-negligible backgrounds, especially, when the 2 nd leading lepton is low- $p_{T}$. Hence, we have to subtract these backgrounds from the Drell-Yan events. Like-sign dilepton events with $\Delta \phi_{\ell_{1} \ell_{2}}>2.8$ (rad) are mainly fake leptons. We estimate the number of Drell-Yan events $\left(N_{\mathrm{DY}}\right)$ by

$$
\begin{gathered}
N_{\mathrm{DY}}=N_{\mathrm{OS}}-N_{\mathrm{LS}} \times R_{\mathrm{OSLS}}, \\
R_{\mathrm{OSLS}}=\frac{\text { The number of fake OS events }}{\text { The number of fake LS events }},
\end{gathered}
$$

where $N_{\mathrm{OS}}$ is the number of oppisite-sign dilepton events, $N_{\mathrm{LS}}$ is the number of likesign dilepton events. Dilepton events have the first leading lepton passing all lepton identification cut and the second leading lepton passing geometrical, kinematical cut. We define the fake Drell-Yan event as the first leading object is passing all lepton selection criteria and the second leading object is satisfied geometrical, kinematical cut, and also required hadronic object criteria (HAD/EM i $0.2, \mathrm{HAD} i, 1.0 \mathrm{GeV}$, and EM i $4 \mathrm{GeV}$ ). If we simply regard LS dilepton events as background, we may underestimate the background events because real dilepton events have charge correlation [58]. We require the leading lepton passing all lepton selections and the event fire the high- $p_{T}$ lepton trigger. Then, we apply our lepton selections to the 2nd leading lepton, as a probe, passing only the geometrical and kinematical selections. The efficiency $\varepsilon$ is defined as

$$
\varepsilon=\frac{N_{\text {after }}}{N_{\text {before }}}
$$

where $N_{\text {before }}$ and $N_{\text {after }}$ are the number of events before and after the cuts, respectively.

\subsubsection{Low- $E_{T}$ Electron Selection Efficiency and Scale Factor}

We estimate the electron selection efficiency and scale factor for low- $E_{T}$ electrons $\left(E_{T}<\right.$ $30 \mathrm{GeV})$ using Drell-Yan events $\left(\Delta \phi_{\ell_{1} \ell_{2}}>2.8\right)$, the events are provided by extracting 
LS dielectron events applied OSLS ratio from OS dielectron events with the invariant mass greater than $20 \mathrm{GeV} / c^{2}$. As mentioned before, the identification scale factor depends on the $E_{T}$ of electron. However, in $6-8 \mathrm{GeV} / c$ region, we can not neglect the amount of fake leptons and leptons from conversion events. Therefore, we deduce efficiencies and scale factors above $8 \mathrm{GeV} / c$ and use the scale factors to the whole low- $E_{T}$ region $\left(6<E_{T}<30 \mathrm{GeV} / c\right)$. The efficiency in each dataset is shown in A.3.1.

\subsubsection{High- $E_{T}$ Electron Selection Efficiency and Scale Factor}

We estimate the electron selection efficiency and scale factor for high- $E_{T}$ electrons $\left(E_{T}>30 \mathrm{GeV}\right)$ using Drell-Yan events within $Z$ mass window $\left(76<M_{e e}<106\right)$ and the background subtraction is same as low- $E_{T}$ electron selection efficiency study (see $\S 4.3 .7)$. The efficiency in each dataset is shown in A.3.1.

\subsubsection{Muon Selection Efficiency and Scale Factor}

We estimate the muon selection efficiency and the scale factor using Drell-Yan events with OSLS ratio to subtract background events. In the muon case, there is no clear muon $p_{T}$ dependence for any muon selection variables. However, for CMU, CMP, BMU, CMIO-CES and CMIO-PES, contaminations from fake muons is too large to neglect. As the case of electrons, we estimate the efficiencies and scale factors above $p_{T}$ greater than $30 \mathrm{GeV} / c$, and use the scale factors to the whole $p_{T}$ region $\left(p_{T} \geq 6 \mathrm{GeV} / c\right)$. The efficiency in each dataset is shown in A.3.2.

\subsubsection{Muon Reconstruction Efficiency and Scale Factor}

We use Drell-Yan event passing $\Delta \phi_{\ell_{1} \ell_{2}}$ cut $(>2.8)$, the difference of $z_{0}$ position criterion for dimuon $\left(\left|z_{0}^{\ell_{1}}-z_{0}^{\ell_{2}}\right|<2 \mathrm{~cm}\right)$ to estimate the muon reconstruction efficiency. we also use OSLS ratio to subtract the background. We have used the high- $p_{T}$ muon trigger samples and the Drell-Yan MC samples. The efficiency in each dataset is shown in A.3.3.

\subsubsection{1 $Z / \gamma^{*} \rightarrow \ell^{+} \ell^{-}$Cross Section}

We measure the $Z / \gamma^{*} \rightarrow \ell^{+} \ell^{-}$cross section for the invariant mass region $66-116 \mathrm{GeV} / c^{2}$ using the trigger efficiencies and the scale factors to validate our estimations. The cross section can be expressed as

$$
\begin{gathered}
\sigma\left(Z / \gamma^{*} \rightarrow \ell^{+} \ell^{-}\right)=\frac{N_{\mathrm{obs}}}{L \cdot \varepsilon}, \\
\varepsilon=\varepsilon_{\mathrm{MC}} \cdot \varepsilon_{\text {trig }} \cdot \mathrm{SF}_{\mathrm{pv}} \cdot \mathrm{SF}_{\ell_{1}} \cdot \mathrm{SF}_{\ell_{2}},
\end{gathered}
$$

where $N_{\text {obs }}$ is the number of observed events, $L$ is the integrated luminosity, $\varepsilon_{\mathrm{MC}}$ is $Z / \gamma^{*} \rightarrow \ell^{+} \ell^{-}$efficiency derived by Drell-Yan MC samples, $\varepsilon_{\text {trig }}$ means lepton trigger 
efficiency, $\mathrm{SF}_{\mathrm{pv}}$ is the scale factor for the primary-vertex cut efficiency, and $\mathrm{SF}_{\ell_{1,2}}$ means the scale factors for the lepton selection and the muon reconstruction. Tables 4.4 through 4.12 show the number of observed events, expected events and cross sections for each dilepton types. We consider the overall difference (4.6\%) as systematic uncertainty for our like-sign dilepton analysis. 
Table 4.4: The number of $Z / \gamma^{*}\left(66<M_{\ell \ell}<116 \mathrm{GeV}\right)$ event and the cross section for CEM-CEM pair. (Note : Quote NNLO cross section $(\sigma=251.3 \pm 5 \mathrm{pb})$ to estimate the significance for cross section.)

\begin{tabular}{ccccccc}
\hline \hline Dataset & $\begin{array}{c}\text { Luminosity } \\
\left(\mathrm{pb}^{-1}\right)\end{array}$ & $\begin{array}{c}\text { Observed } \\
(\text { Events })\end{array}$ & $\begin{array}{c}\text { Acceptance } \\
(\%)\end{array}$ & $\begin{array}{c}\text { Cross-section } \\
(\mathrm{pb})\end{array}$ & $\begin{array}{c}\text { Expect } \\
(\text { Events })\end{array}$ & Significance \\
\hline Od & 350 & $4977 \pm 71$ & $5.48 \pm 0.35$ & $260 \pm 17$ & $4817 \pm 324$ & 0.47 \\
Oh & 398 & $5700 \pm 75$ & $5.71 \pm 0.36$ & $251 \pm 16$ & $5717 \pm 379$ & -0.044 \\
Oi & 619 & $8277 \pm 91$ & $5.39 \pm 0.34$ & $248 \pm 16$ & $8387 \pm 551$ & -0.20 \\
Oj & 951 & $10873 \pm 104$ & $4.86 \pm 0.30$ & $235 \pm 15$ & $11618 \pm 758$ & -1.0 \\
Ok & 514 & $6213 \pm 79$ & $4.85 \pm 0.31$ & $249 \pm 16$ & $6264 \pm 414$ & -0.12 \\
Om & 3207 & $32261 \pm 180$ & $4.12 \pm 0.25$ & $244 \pm 15$ & $33195 \pm 2138$ & -0.45 \\
Op & 3329 & $29659 \pm 172$ & $4.09 \pm 0.25$ & $218 \pm 13$ & $34177 \pm 2205$ & -2.3 \\
\hline \hline
\end{tabular}

Table 4.5: The number of $Z / \gamma^{*}\left(66<M_{\ell \ell}<116 \mathrm{GeV}\right)$ event and the cross section for CMUP-CMUP pair. (Note : Quote NNLO cross section $(\sigma=251.3 \pm 5 \mathrm{pb})$ to estimate the significance for cross section.)

\begin{tabular}{ccccccc}
\hline \hline Dataset & $\begin{array}{c}\text { Luminosity } \\
\left(\mathrm{pb}^{-1}\right)\end{array}$ & $\begin{array}{c}\text { Observed } \\
(\text { Events })\end{array}$ & $\begin{array}{c}\text { Acceptance } \\
(\%)\end{array}$ & $\begin{array}{c}\text { Cross-section } \\
(\mathrm{pb})\end{array}$ & $\begin{array}{c}\text { Expect } \\
(\text { Events })\end{array}$ & Significance \\
\hline Od & 350 & $1478 \pm 38$ & $1.62 \pm 0.12$ & $261 \pm 20$ & $1425 \pm 105$ & 0.46 \\
$0 \mathrm{~h}$ & 398 & $1656 \pm 41$ & $1.57 \pm 0.12$ & $266 \pm 22$ & $1567 \pm 128$ & 0.63 \\
$0 \mathrm{i}$ & 619 & $2531 \pm 50$ & $1.52 \pm 0.11$ & $269 \pm 20$ & $2361 \pm 174$ & 0.88 \\
$0 \mathrm{j}$ & 951 & $3273 \pm 57$ & $1.45 \pm 0.11$ & $238 \pm 19$ & $3462 \pm 282$ & -0.69 \\
$0 \mathrm{k}$ & 513 & $1772 \pm 42$ & $1.272 \pm 0.094$ & $271 \pm 21$ & $1641 \pm 126$ & 0.92 \\
$0 \mathrm{~m}$ & 3207 & $9061 \pm 95$ & $1.124 \pm 0.071$ & $251 \pm 16$ & $9060 \pm 598$ & 0.0012 \\
$\mathrm{Op}$ & 3331 & $8002 \pm 89$ & $1.083 \pm 0.071$ & $222 \pm 15$ & $9061 \pm 624$ & -1.9 \\
\hline \hline
\end{tabular}

Table 4.6: The number of $Z / \gamma^{*}\left(66<M_{\ell \ell}<116 \mathrm{GeV}\right)$ event and the cross section for CMX-CMUP pair. (Note: Quote NNLO cross section $(\sigma=251.3 \pm 5 \mathrm{pb})$ to estimate the significance for cross section.)

\begin{tabular}{ccccccc}
\hline \hline Dataset & $\begin{array}{c}\text { Luminosity } \\
\left(\mathrm{pb}^{-1}\right)\end{array}$ & $\begin{array}{c}\text { Observed } \\
(\text { Events })\end{array}$ & $\begin{array}{c}\text { Acceptance } \\
(\%)\end{array}$ & $\begin{array}{c}\text { Cross-section } \\
(\mathrm{pb})\end{array}$ & $\begin{array}{c}\text { Expect } \\
(\text { Events })\end{array}$ & Significance \\
\hline Od & 350 & $718 \pm 27$ & $0.727 \pm 0.051$ & $282 \pm 22$ & $639 \pm 47$ & 1.4 \\
Oh & 398 & $1054 \pm 32$ & $0.996 \pm 0.074$ & $266 \pm 21$ & $997 \pm 76$ & 0.66 \\
$0 \mathrm{i}$ & 619 & $1461 \pm 38$ & $0.959 \pm 0.076$ & $246 \pm 20$ & $1493 \pm 121$ & -0.25 \\
$\mathrm{Oj}_{\mathrm{j}}$ & 951 & $2047 \pm 45$ & $0.899 \pm 0.065$ & $239 \pm 18$ & $2149 \pm 161$ & -0.64 \\
ok & 513 & $969 \pm 31$ & $0.844 \pm 0.060$ & $224 \pm 18$ & $1089 \pm 81$ & -1.5 \\
Om & 3207 & $5851 \pm 76$ & $0.754 \pm 0.048$ & $242 \pm 16$ & $6080 \pm 402$ & -0.58 \\
Op & 3331 & $4898 \pm 70$ & $0.733 \pm 0.047$ & $201 \pm 13$ & $6134 \pm 416$ & -3.6 \\
\hline \hline
\end{tabular}


Table 4.7: The number of $Z / \gamma^{*}\left(66<M_{\ell \ell}<116 \mathrm{GeV}\right)$ event and the cross section for CMUP-CMX pair. (Note: Quote NNLO cross section $(\sigma=251.3 \pm 5 \mathrm{pb})$ to estimate the significance for cross section.)

\begin{tabular}{ccccccc}
\hline \hline Dataset & $\begin{array}{c}\text { Luminosity } \\
\left(\mathrm{pb}^{-1}\right)\end{array}$ & $\begin{array}{c}\text { Observed } \\
(\text { Events })\end{array}$ & $\begin{array}{c}\text { Acceptance } \\
(\%)\end{array}$ & $\begin{array}{c}\text { Cross-section } \\
(\mathrm{pb})\end{array}$ & $\begin{array}{c}\text { Expect } \\
(\text { Events })\end{array}$ & Significance \\
\hline $\mathrm{Od}$ & 350 & $649 \pm 25$ & $0.778 \pm 0.055$ & $238 \pm 19$ & $684 \pm 50$ & -0.65 \\
$\mathrm{Oh}$ & 398 & $1108 \pm 33$ & $1.049 \pm 0.078$ & $265 \pm 21$ & $1050 \pm 80$ & 0.63 \\
$\mathrm{O} \mathrm{i}$ & 619 & $1516 \pm 39$ & $1.020 \pm 0.081$ & $240 \pm 20$ & $1587 \pm 129$ & -0.55 \\
$0 \mathrm{j}$ & 951 & $2076 \pm 46$ & $0.966 \pm 0.070$ & $226 \pm 17$ & $2309 \pm 173$ & -1.4 \\
$0 \mathrm{k}$ & 513 & $1081 \pm 33$ & $0.887 \pm 0.063$ & $237 \pm 18$ & $1145 \pm 85$ & -0.73 \\
$0 \mathrm{~m}$ & 3207 & $5971 \pm 77$ & $0.793 \pm 0.050$ & $235 \pm 15$ & $6391 \pm 422$ & -1.0 \\
$\mathrm{Op}$ & 3331 & $5232 \pm 72$ & $0.770 \pm 0.050$ & $204 \pm 14$ & $6447 \pm 437$ & -3.3 \\
\hline \hline
\end{tabular}

Table 4.8: The number of $Z / \gamma^{*}\left(66<M_{\ell \ell}<116 \mathrm{GeV}\right)$ event and the cross section for CMX-CMX pair. (Note: Quote NNLO cross section $(\sigma=251.3 \pm 5 \mathrm{pb})$ to estimate the significance for cross section.)

\begin{tabular}{ccccccc}
\hline \hline Dataset & $\begin{array}{c}\text { Luminosity } \\
\left(\mathrm{pb}^{-1}\right)\end{array}$ & $\begin{array}{c}\text { Drell-Yan } \\
(\text { Events })\end{array}$ & $\begin{array}{c}\text { Acceptance } \\
(\%)\end{array}$ & $\begin{array}{c}\text { Cross-section } \\
(\mathrm{pb})\end{array}$ & $\begin{array}{c}\text { Expect } \\
(\text { Events })\end{array}$ & Significance \\
\hline Od & 350 & $441 \pm 21$ & $0.423 \pm 0.029$ & $298 \pm 25$ & $372 \pm 27$ & 1.8 \\
Oh & 398 & $707 \pm 27$ & $0.670 \pm 0.046$ & $265 \pm 21$ & $671 \pm 48$ & 0.63 \\
Oi & 619 & $1115 \pm 33$ & $0.652 \pm 0.057$ & $276 \pm 25$ & $1014 \pm 90$ & 0.97 \\
Oj & 951 & $1465 \pm 38$ & $0.603 \pm 0.040$ & $255 \pm 18$ & $1442 \pm 100$ & 0.22 \\
Ok & 513 & $755 \pm 27$ & $0.583 \pm 0.040$ & $252 \pm 20$ & $752 \pm 54$ & 0.053 \\
Om & 3207 & $4457 \pm 67$ & $0.528 \pm 0.033$ & $263 \pm 17$ & $4258 \pm 281$ & 0.66 \\
Op & 3331 & $4145 \pm 64$ & $0.517 \pm 0.033$ & $241 \pm 16$ & $4330 \pm 288$ & -0.65 \\
\hline \hline
\end{tabular}

Table 4.9: The number of $Z / \gamma^{*}\left(66<M_{\ell \ell}<116 \mathrm{GeV}\right)$ event and the cross section for CMUP-(CMIOCES or CMIOPES) pair. (Note : Quote NNLO cross section $(\sigma=251.3$ $\pm 5 \mathrm{pb})$ to estimate the significance for cross section.)

\begin{tabular}{ccccccc}
\hline \hline Dataset & $\begin{array}{c}\text { Luminosity } \\
\left(\mathrm{pb}^{-1}\right)\end{array}$ & $\begin{array}{c}\text { Observed } \\
(\text { Events })\end{array}$ & $\begin{array}{c}\text { Acceptance } \\
(\%)\end{array}$ & $\begin{array}{c}\text { Cross-section } \\
(\mathrm{pb})\end{array}$ & $\begin{array}{c}\text { Expect } \\
(\text { Events })\end{array}$ & Significance \\
\hline Od & 350 & $368 \pm 19$ & $0.565 \pm 0.047$ & $186 \pm 18$ & $496 \pm 43$ & -3.4 \\
Oh & 398 & $330 \pm 18$ & $0.324 \pm 0.029$ & $256 \pm 27$ & $324 \pm 30$ & 0.17 \\
Oi & 619 & $511 \pm 23$ & $0.302 \pm 0.024$ & $274 \pm 25$ & $469 \pm 39$ & 0.87 \\
Oj & 951 & $612 \pm 25$ & $0.255 \pm 0.021$ & $252 \pm 23$ & $610 \pm 52$ & 0.028 \\
Ok & 513 & $312 \pm 18$ & $0.263 \pm 0.064$ & $231 \pm 58$ & $340 \pm 83$ & -0.35 \\
Om & 3207 & $1821 \pm 43$ & $0.233 \pm 0.016$ & $244 \pm 17$ & $1878 \pm 131$ & -0.42 \\
Op & 3331 & $1660 \pm 41$ & $0.217 \pm 0.015$ & $229 \pm 17$ & $1819 \pm 131$ & -1.2 \\
\hline \hline
\end{tabular}


Table 4.10: The number of $Z / \gamma^{*}\left(66<M_{\ell \ell}<116 \mathrm{GeV}\right)$ event and the cross section for CMX-(CMIOCES or CMIOPES) pair. (Note : Quote NNLO cross section $(\sigma=251.3$ $\pm 5 \mathrm{pb})$ to estimate the significance for cross section.)

\begin{tabular}{ccccccc}
\hline \hline Dataset & $\begin{array}{c}\text { Luminosity } \\
\left(\mathrm{pb}^{-1}\right)\end{array}$ & $\begin{array}{c}\text { Observed } \\
(\text { Events })\end{array}$ & $\begin{array}{c}\text { Acceptance } \\
(\%)\end{array}$ & $\begin{array}{c}\text { Cross-section } \\
(\mathrm{pb})\end{array}$ & $\begin{array}{c}\text { Expect } \\
(\text { Events })\end{array}$ & Significance \\
\hline Od & 350 & $193 \pm 14$ & $0.222 \pm 0.018$ & $249 \pm 27$ & $195 \pm 17$ & -0.093 \\
Oh & 398 & $216 \pm 15$ & $0.207 \pm 0.017$ & $262 \pm 28$ & $207 \pm 18$ & 0.38 \\
Oi & 619 & $306 \pm 17$ & $0.191 \pm 0.017$ & $259 \pm 27$ & $297 \pm 27$ & 0.28 \\
Oj & 951 & $431 \pm 21$ & $0.166 \pm 0.012$ & $274 \pm 24$ & $396 \pm 31$ & 0.90 \\
Ok & 513 & $248 \pm 16$ & $0.170 \pm 0.040$ & $285 \pm 70$ & $219 \pm 52$ & 0.48 \\
Om & 3207 & $1310 \pm 36$ & $0.152 \pm 0.010$ & $269 \pm 20$ & $1222 \pm 86$ & 0.89 \\
Op & 3331 & $1135 \pm 34$ & $0.1430 \pm 0.0098$ & $238 \pm 18$ & $1197 \pm 85$ & -0.71 \\
\hline \hline
\end{tabular}

Table 4.11: The number of $Z / \gamma^{*}\left(66<M_{\ell \ell}<116 \mathrm{GeV}\right)$ event and the cross section for CMUP-(CMU, CMP, or BMU) pair. (Note : Quote NNLO cross section $(\sigma=251.3 \pm$ $5 \mathrm{pb})$ to estimate the significance for cross section.)

\begin{tabular}{ccccccc}
\hline \hline Dataset & $\begin{array}{c}\text { Luminosity } \\
\left(\mathrm{pb}^{-1}\right)\end{array}$ & $\begin{array}{c}\text { Observed } \\
(\text { Events })\end{array}$ & $\begin{array}{c}\text { Acceptance } \\
(\%)\end{array}$ & $\begin{array}{c}\text { Cross-section } \\
(\mathrm{pb})\end{array}$ & $\begin{array}{c}\text { Expect } \\
(\text { Events })\end{array}$ & Significance \\
\hline Od & 350 & $743 \pm 27$ & $0.803 \pm 0.057$ & $264 \pm 21$ & $706 \pm 52$ & 0.60 \\
Oh & 398 & $846 \pm 29$ & $0.781 \pm 0.067$ & $272 \pm 25$ & $782 \pm 69$ & 0.81 \\
$0 \mathrm{i}$ & 619 & $1224 \pm 35$ & $0.763 \pm 0.052$ & $259 \pm 19$ & $1187 \pm 84$ & 0.40 \\
$0 \mathrm{j}$ & 951 & $1626 \pm 40$ & $0.671 \pm 0.060$ & $255 \pm 24$ & $1604 \pm 147$ & 0.14 \\
Ok & 513 & $935 \pm 31$ & $0.637 \pm 0.056$ & $286 \pm 27$ & $822 \pm 74$ & 1.3 \\
Om & 3207 & $4997 \pm 71$ & $0.579 \pm 0.037$ & $269 \pm 17$ & $4664 \pm 310$ & 0.99 \\
Op & 3331 & $4708 \pm 69$ & $0.559 \pm 0.037$ & $253 \pm 17$ & $4681 \pm 323$ & 0.081 \\
\hline \hline
\end{tabular}

Table 4.12: The number of $Z / \gamma^{*}\left(66<M_{\ell \ell}<116 \mathrm{GeV}\right)$ event and the cross section for CMX-(CMU, CMP, or BMU) pair. (Note : Quote NNLO cross section $(\sigma=251.3 \pm$ $5 \mathrm{pb}$ ) to estimate the significance for cross section.)

\begin{tabular}{ccccccc}
\hline \hline Dataset & $\begin{array}{c}\text { Luminosity } \\
\left(\mathrm{pb}^{-1}\right)\end{array}$ & $\begin{array}{c}\text { Observed } \\
(\text { Events })\end{array}$ & $\begin{array}{c}\text { Acceptance } \\
(\%)\end{array}$ & $\begin{array}{c}\text { Cross-section } \\
(\mathrm{pb})\end{array}$ & $\begin{array}{c}\text { Expect } \\
(\text { Events })\end{array}$ & Significance \\
\hline $\mathrm{Od}$ & 350 & $369 \pm 19$ & $0.392 \pm 0.028$ & $269 \pm 24$ & $345 \pm 25$ & 0.74 \\
$\mathrm{Oh}$ & 398 & $543 \pm 23$ & $0.510 \pm 0.041$ & $267 \pm 24$ & $511 \pm 42$ & 0.64 \\
$0 \mathrm{i}$ & 619 & $823 \pm 29$ & $0.494 \pm 0.037$ & $269 \pm 22$ & $769 \pm 60$ & 0.77 \\
$0 \mathrm{j}$ & 951 & $1007 \pm 32$ & $0.434 \pm 0.035$ & $244 \pm 21$ & $1037 \pm 86$ & -0.34 \\
$\mathrm{Ok}$ & 513 & $613 \pm 25$ & $0.429 \pm 0.036$ & $278 \pm 26$ & $553 \pm 48$ & 1.0 \\
$0 \mathrm{~m}$ & 3207 & $3395 \pm 58$ & $0.395 \pm 0.025$ & $268 \pm 18$ & $3185 \pm 212$ & 0.91 \\
$\mathrm{Op}$ & 3331 & $3097 \pm 56$ & $0.385 \pm 0.025$ & $242 \pm 16$ & $3219 \pm 217$ & -0.56 \\
\hline \hline
\end{tabular}


Table 4.13: Summary of observed events, expected events, and Significance of $Z \rightarrow \ell^{ \pm} \ell^{\mp}$ pairs in period 0 through 38. Note: CMIO means CMIO-CES or CMIO-PES, OM means CMU, CMP, or BMU.

\begin{tabular}{cccc}
\hline Dilepton Type & Observed Events $(\mathrm{O})$ & Expected Events $(\mathrm{E})$ & $(\mathrm{O}-\mathrm{E}) / \mathrm{O}$ \\
\hline CEM-CEM & 97959 & $104175 \pm 3276$ & -0.063 \\
CMUP-CMUP & 27773 & $28577 \pm 948$ & -0.028 \\
CMX-CMUP & 16998 & $18580 \pm 623$ & -0.093 \\
CMUP-CMX & 17633 & $19612 \pm 657$ & -0.11 \\
CMX-CMX & 13085 & $12837 \pm 431$ & 0.018 \\
CMUP-CMIO & 5614 & $5936 \pm 219$ & -0.057 \\
CMX-CMIO & 3839 & $3732 \pm 139$ & 0.027 \\
CMUP-OM & 15079 & $14445 \pm 491$ & 0.042 \\
CMX-OM & 9846 & $9618 \pm 99$ & 0.023 \\
\hline Overall & 207827 & $217517 \pm 3599$ & -0.046 \\
\hline \hline
\end{tabular}




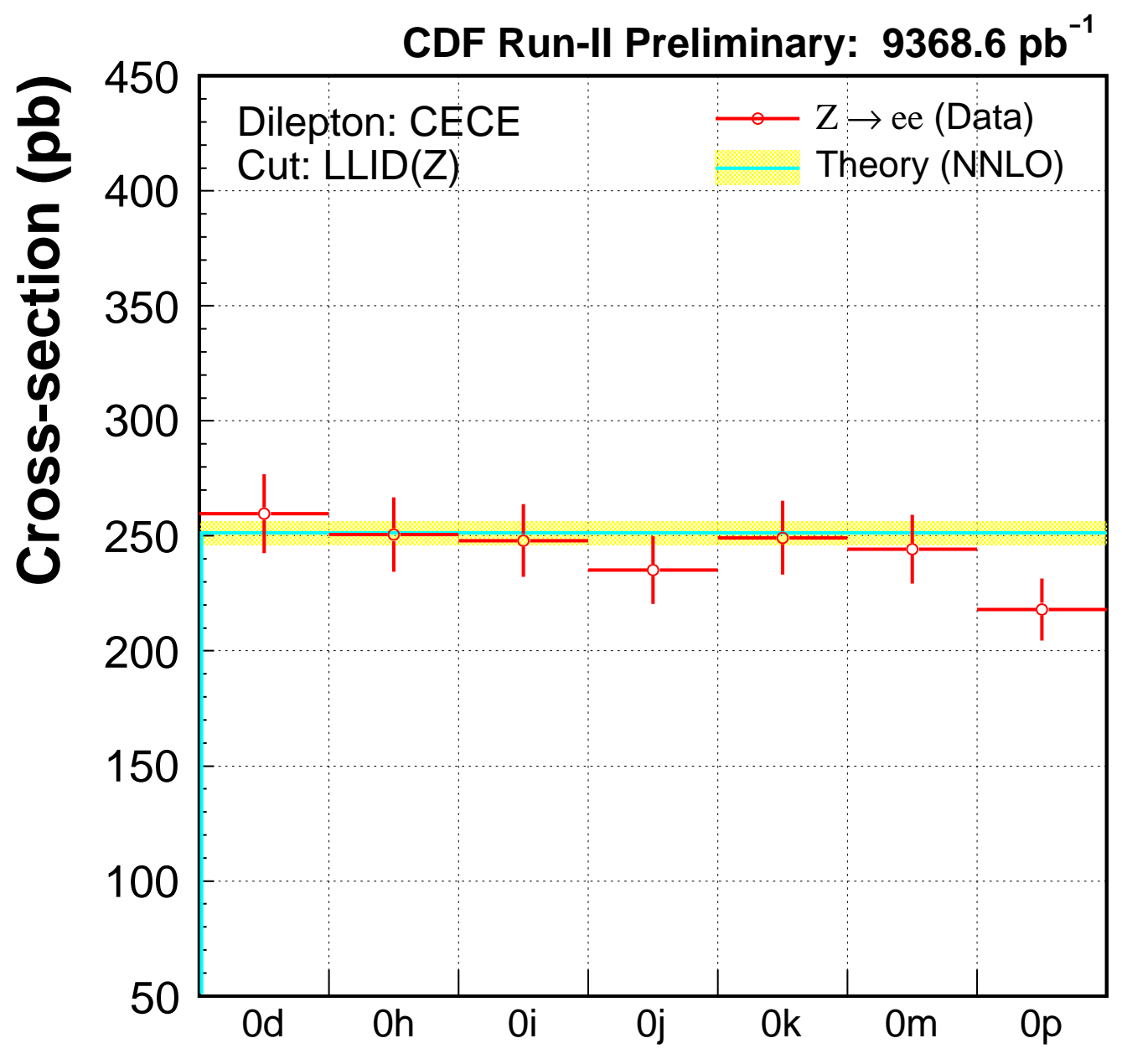

Figure 4.33: $Z / \gamma^{*} \rightarrow e^{+} e^{-}$Cross Section to validate the trigger efficiency and scale factors. 

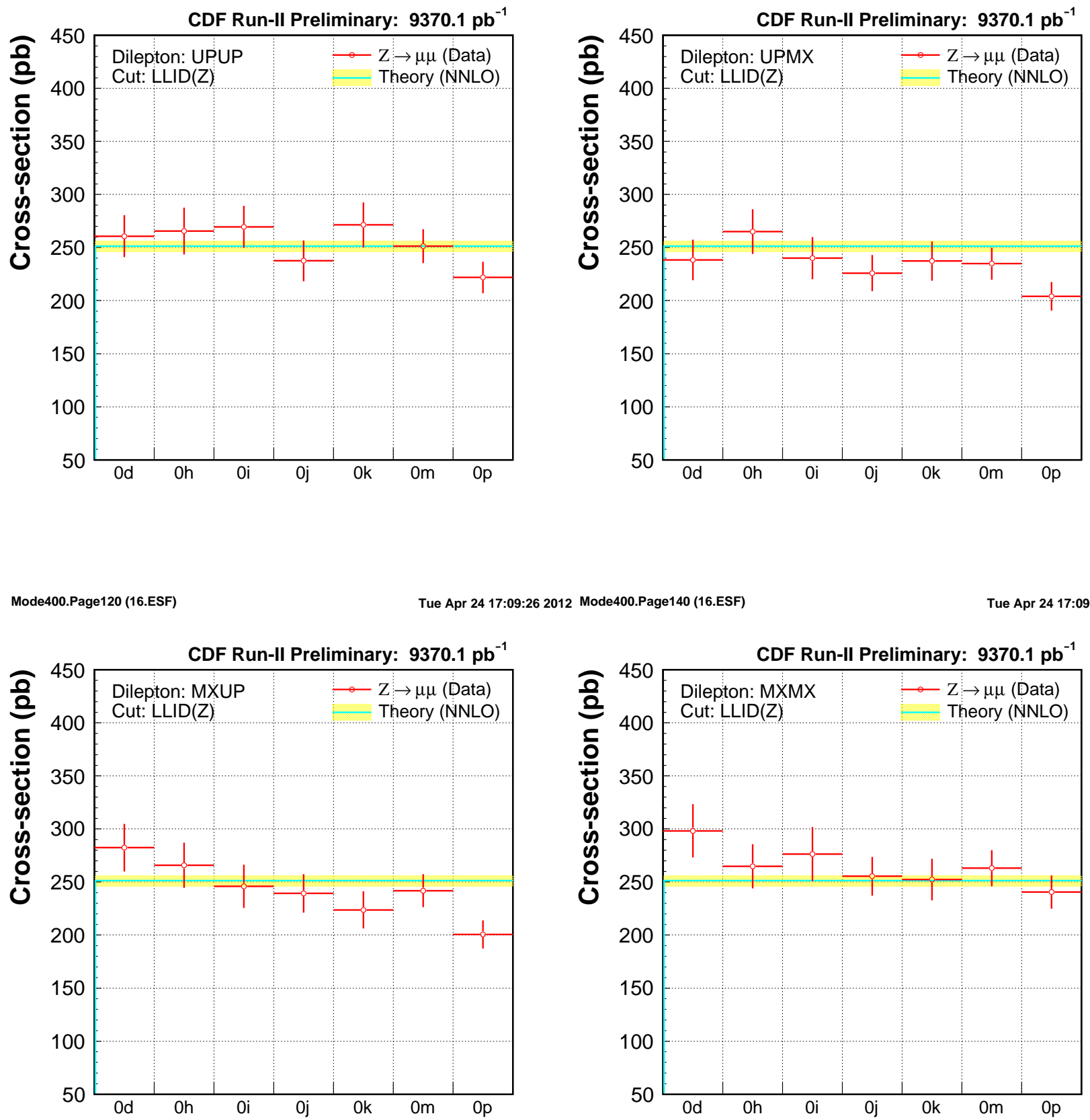

Figure 4.34: $Z / \gamma^{*} \rightarrow \mu^{+} \mu^{-}$Cross Section to validate the trigger efficiency and scale factors. The left upper plots is CMUP-CMUP combination, the right upper plots is CMUP-CMX combination, the left lower plots is CMX-CMUP combination, and the right upper plots is CMX-CMX combination. 

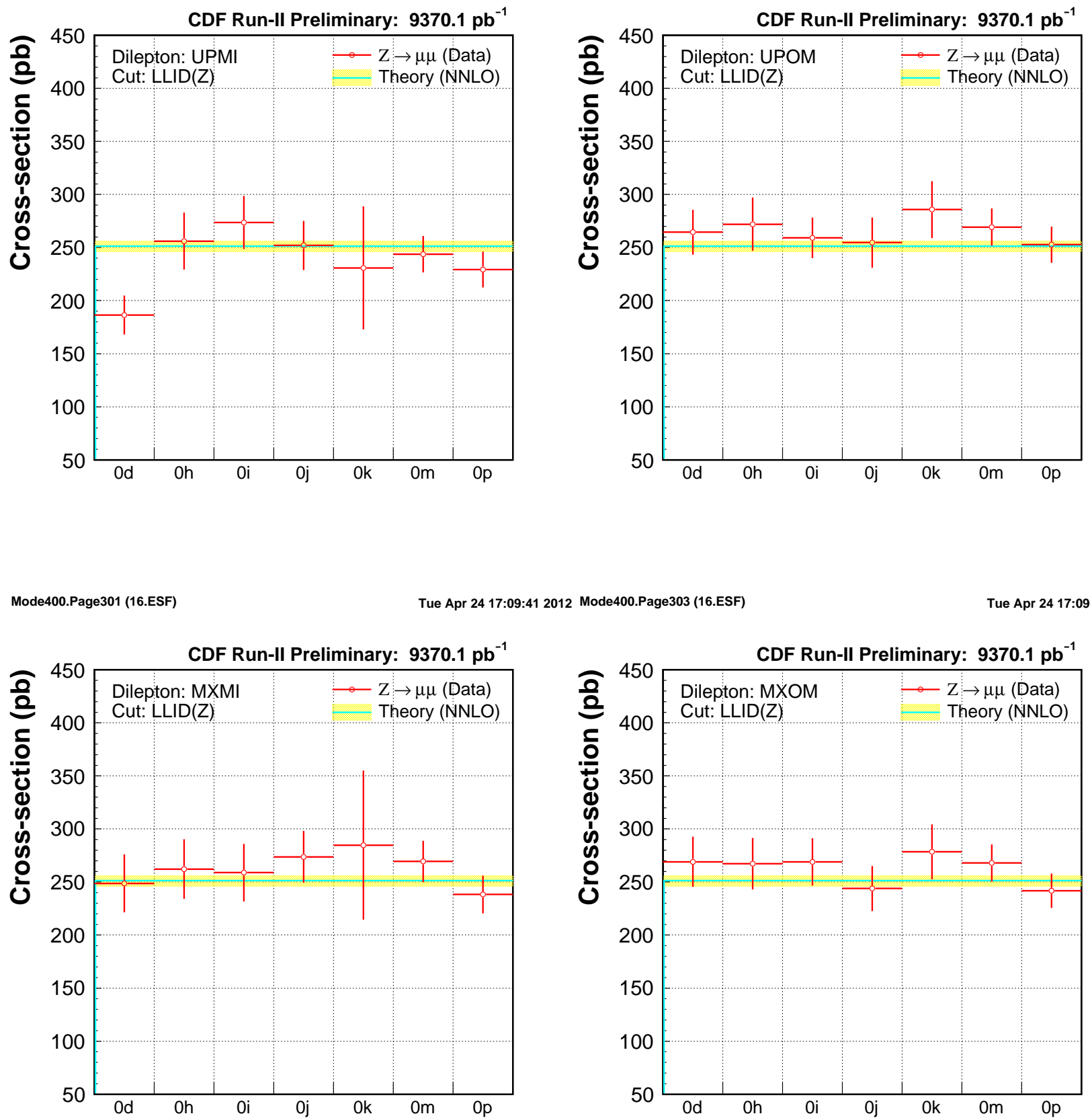

Figure 4.35: $Z / \gamma^{*} \rightarrow \mu^{+} \mu^{-}$Cross Section to validate the trigger efficiency and scale factors. The left upper plots is CMUP-(CMIOCES or CMIOPES) combination, the right upper plots is $\mathrm{CMUP}-(\mathrm{CMU}, \mathrm{CMP}$, or $\mathrm{BMU})$ combination, the left lower plots is CMX-(CMIOCES or CMIOPES) combination, and the right upper plots is CMX(CMU, CMP, or BMU) combination. 


\subsection{Expected Backgrounds}

To validate our estimation, we use three pilot regions: lepton-ID side-band region, opposite-sign region, and zero-silicon region. We call the 1st lepton passing all selection criteria and the 2nd lepton passing all selection except for the lepton identification as the side-band region. The opposite-sign region is the same as the signal region except for the charge combination of dilepton. The zero-silicon region is defined by

- 1st lepton with nominal silicon hit requirement

- 2nd lepton with zero-silicon hit requirement

- Silicon fiducial cut, that is, 2nd lepton passing through SVX 5 layers and $\left|z_{0}\right|<$ $47.25 \mathrm{~cm}$ at $R=10.645$.

The expected number of events and data in pilot regions and our signal regions are summarized in Table 4.14. In this table, the expected number of the Higgs boson is also shown, and the details are shown in chapter 5 .

There are no significant discrepancies between the data and the background expectations for each region. In the signal region, the number of total observed events is 624 events, while the background expectation is $696.1 \pm 52.8$ events. 
Table 4.14: Background expectation and observed number of events for side-band, zero-silicon, opposite-sign and like-sign(signal) dilepton events.

\begin{tabular}{c|c|c|c|c} 
& Lepton ID Side-Band & Zero Silicon & OS & Signal \\
\hline \hline Fakes & 4493.9 & 15.7 & 674.8 & 631.9 \\
Photon-conversions & 123.1 & 91.7 & 192.5 & 49.5 \\
\hline Total & $4616.9 \pm 595.6$ & $107.4 \pm 13.2$ & $867.3 \pm 114.7$ & $681.4 \pm 52.8$ \\
\hline$Z / \gamma^{*} \rightarrow e e$ & - & - & 19841.4 & - \\
$Z / \gamma^{*} \rightarrow \mu \mu$ & - & - & 30327.3 & - \\
$Z / \gamma^{*} \rightarrow \tau \tau$ & - & - & 4071.3 & - \\
$t \bar{t}$ & - & - & 269.2 & - \\
$W W$ & - & - & 399.2 & - \\
$W Z$ & 2.1 & - & 27.3 & 13.1 \\
$Z Z$ & 0.4 & - & 23.7 & 1.7 \\
\hline \hline Total MC & $2.5 \pm 0.3$ & - & $54959.4 \pm 4159.2$ & $14.8 \pm 1.7$ \\
\hline Fermiophobic higgs (Wh110) & $0.88 \pm 0.10$ & - & $6.31 \pm 0.71$ & $5.09 \pm 0.59$ \\
Fermiophobic higgs (Zh110) & $0.10 \pm 0.01$ & - & $8.33 \pm 0.27$ & $0.53 \pm 0.06$ \\
\hline Fermiophobic Total (110) & $0.98 \pm 0.10$ & - & $2.46 \pm 0.28$ & $1.51 \pm 0.17$ \\
\hline \hline SM higgs (Wh160) & $0.19 \pm 0.02$ & - & $1.15 \pm 0.13$ & $0.18 \pm 0.02$ \\
\hline SM higgs (Zh160) & $0.028 \pm 0.003$ & - & $3.61 \pm 0.31$ & $1.69 \pm 0.17$ \\
\hline SM Total (160) & $0.21 \pm 0.02$ & $107.4 \pm 13.2$ & $55826.7 \pm 4214.6$ & $696.1 \pm 52.8$ \\
\hline \hline Total expected & $4619.4 \pm 595.6$ & 127 & 51243 & 624 \\
\hline Data & 4598 & & & \\
\hline \hline
\end{tabular}




\section{Chapter 5}

\section{Search for the Higgs Boson}

So far, the background events in like-sign dilepton events have been described in section 4. In this chapter, the events of the Higgs boson is explained. The search for the Higgs boson is very challenging due to the small production cross section. For example, $\sigma(p \bar{p} \rightarrow W h)=0.048 \mathrm{pb}$ on $m_{H}=160 \mathrm{GeV} / c^{2}$, while $W Z$ production is $\sigma(p \bar{p} \rightarrow W Z)=3.96 \mathrm{pb}$. In order to improve the sensitivity for the Higgs boson, we employ a tequnique based on multivariate analysis. In the case of lepton identifications, we have already used one of multivariate tequniques, based on likelihood, and set the cut values to reject backgrounds as possible. After all selection cuts, we employ the other tequnique, "Boosted Decision Trees", described in the following sections.

\subsection{Expected Number of Events for the Higgs Bo- son}

Expected number of events after passing LS-dilepton selection is estimated by using Higgs MCs which are generated by PYTHIA assuming the mass of the Higgs boson between from 110 to $200 \mathrm{GeV} / c^{2}$ in $10 \mathrm{GeV} / c^{2}$ steps. The standard model does not tell us the mass of Higgs boson, but predicts the existence and other properties. The cross section and branching fraction on each mass is shown in Table 5.1. The expected number of events is summarized in Table 5.2. 
Table 5.1: Theoretical cross sections and branching fractions on each mass of the Higgs boson.

\begin{tabular}{ccccc}
\hline $\begin{array}{c}\text { Higgs Mass } \\
\left(\mathrm{GeV} / c^{2}\right)\end{array}$ & $\begin{array}{c}\sigma(p \bar{p} \rightarrow W h) \\
(\mathrm{fb})\end{array}$ & $\begin{array}{c}\sigma(p \bar{p} \rightarrow Z h) \\
(\mathrm{fb})\end{array}$ & $\begin{array}{c}B_{F}(h \rightarrow W W) \\
(\text { Fermiophobic higgs })\end{array}$ & $\begin{array}{c}B_{F}(h \rightarrow W W) \\
(\mathrm{SM} \mathrm{higgs})\end{array}$ \\
\hline 110 & 203.7 & 120.2 & 0.85 & 0.05 \\
120 & 150.1 & 90.2 & 0.87 & 0.14 \\
130 & 112.0 & 68.5 & 0.87 & 0.30 \\
140 & 84.6 & 52.7 & 0.87 & 0.50 \\
150 & 64.4 & 40.8 & 0.89 & 0.70 \\
160 & 48.5 & 31.4 & 0.95 & 0.91 \\
170 & 38.5 & 25.3 & 0.97 & 0.96 \\
180 & 30.1 & 20.0 & 0.94 & 0.93 \\
190 & 24.0 & 16.1 & 0.79 & 0.79 \\
200 & 19.1 & 13.0 & 0.74 & 0.74 \\
\hline \hline
\end{tabular}

Table 5.2: Expected number of events for signal passing baseline event selections (FP: Fermiophobic Higgs).

\begin{tabular}{|c|c|c|c|c|c|c|}
\hline $\begin{array}{c}\text { Higgs Mass } \\
\left(\mathrm{GeV} / c^{2}\right)\end{array}$ & $\begin{array}{c}W h \\
\text { Expected Events } \\
(\mathrm{FP})\end{array}$ & $\begin{array}{c}W h \\
\text { Expected Events } \\
(\mathrm{SM})\end{array}$ & $\begin{array}{c}h \\
\text { Expected Events } \\
(\mathrm{FP})\end{array}$ & $\begin{array}{c}h \\
\text { Expected Events } \\
(\mathrm{SM})\end{array}$ & $\begin{array}{l}\text { Total } \\
(\mathrm{FP})\end{array}$ & $\begin{array}{l}\text { Total } \\
(\mathrm{SM})\end{array}$ \\
\hline 110 & $5.1 \pm 0.6$ & $0.29 \pm 0.03$ & $0.53 \pm 0.06$ & $0.030 \pm 0.004$ & $5.6 \pm 0.6$ & $0.32 \pm 0.03$ \\
\hline 120 & $4.0 \pm 0.5$ & $0.66 \pm 0.08$ & $0.43 \pm 0.05$ & $0.07 \pm 0.01$ & $4.4 \pm 0.7$ & $0.73 \pm 0.08$ \\
\hline 130 & $3.2 \pm 0.4$ & $1.1 \pm 0.1$ & $0.36 \pm 0.04$ & $0.13 \pm 0.01$ & $3.6 \pm 0.4$ & $1.2 \pm 0.1$ \\
\hline 140 & $2.6 \pm 0.3$ & $1.5 \pm 0.2$ & $0.30 \pm 0.03$ & $0.17 \pm 0.02$ & $2.9 \pm 0.3$ & $1.7 \pm 0.2$ \\
\hline 150 & $2.1 \pm 0.2$ & $1.6 \pm 0.2$ & $0.24 \pm 0.03$ & $0.19 \pm 0.02$ & $2.3 \pm 0.2$ & $1.8 \pm 0.2$ \\
\hline 160 & $1.6 \pm 0.2$ & $1.5 \pm 0.2$ & $0.18 \pm 0.02$ & $0.18 \pm 0.02$ & $1.8 \pm 0.2$ & $1.7 \pm 0.1$ \\
\hline 170 & $1.3 \pm 0.1$ & $1.3 \pm 0.1$ & $0.15 \pm 0.02$ & $0.15 \pm 0.02$ & $1.5 \pm 0.1$ & $1.5 \pm 0.1$ \\
\hline 180 & $1.0 \pm 0.1$ & $1.0 \pm 0.1$ & $0.12 \pm 0.01$ & $0.12 \pm 0.01$ & $1.1 \pm 0.1$ & $1.1 \pm 0.1$ \\
\hline 190 & $0.75 \pm 0.08$ & $0.75 \pm 0.08$ & $0.09 \pm 0.01$ & $0.09 \pm 0.01$ & $0.84 \pm 0.08$ & $0.84 \pm 0.08$ \\
\hline 200 & $0.59 \pm 0.07$ & $0.59 \pm 0.07$ & $0.07 \pm 0.01$ & $0.07 \pm 0.01$ & $0.66 \pm 0.07$ & $0.66 \pm 0.07$ \\
\hline
\end{tabular}

\subsection{Boosted Decision Trees Discriminant}

The number of background expectation is 696.1 events passing LS-dilepton selections, while the signal is 5.62 for the fermiophobic Higgs boson on $m_{H}=110 \mathrm{GeV} / c^{2}$. The search for small signal events in large background has become essential to use the available information in the events as possible to improve the sensitivity of the Higgs boson. The multivariate data analysis is commonly used to extract information. In this search, "Boosted Decision Trees" (BDT) technique which is one of the multivariate data analyses is employed $[64,65]$. A Decision tree is a binary tree structured classifier such 
as Figure 5.1. "S" means signal, "B" means background, and the terminal nodes are called "leaves". The name of S or B in terminal nodes depends on the majority of events in each node. The tree structure is built up by repeatedly splitting the given events to regions that are eventually classified as signal or background. The samples used to construct a decision tree are called training samples. A shortcoming of a decision tree has instability for the classifier response due to statistical fluctuation in the samples. For example, if two input variables such as $E_{T}$ and $p_{T}$ exhibit similar separation power, the variables are handled as almost like one variable. In such a case the whole tree structure is altered below this node. This problem is overcome by "boosting" algorithm. The boosting constructs a forest of decision trees with modifing weights event by event. As a result, the statistical stability for the classifier increases, and the boosting improves the separation performance compared with one single decision tree.

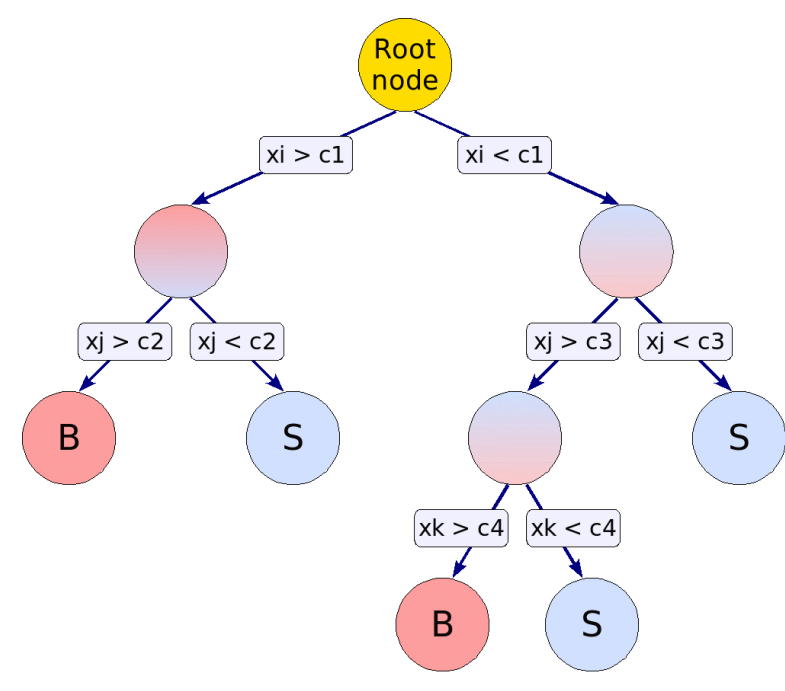

Figure 5.1: Schematic view of a decision tree.

\subsubsection{Decision Tree}

The decision tree is built up under the splitting criteria on each node. The splitting procedure is repeated until the whole tree is built. The split is determined by finding the variable and corresponding cut value that provides the best separation between signal and background. The node splitting is stopped at the time that the node is reached the required minimum number of events. The leaf nodes are classified as signal or background according to the majority of events in the node. The employed splitting criterion is "Gini-Index" to build the decision trees in this thesis. The Gini-Index is defined as

$$
i_{\mathrm{G}}=p(1-p)
$$


$p$ is a purity in a node defined as follows,

$$
p=\frac{\sum_{s} w_{s}}{\sum_{s} w_{s}+\sum_{b} w_{b}}
$$

where $\sum_{s}$ is the sum over signal events and $\sum_{b}$ is the sum over background events in a node, assuming each event is weighted by a value of $w_{i}$, so $p(1-p)$ is 0 if the samples is pure signal or pure background. The criterion is to maximize

$$
i_{\mathrm{G}}(\text { parent })-i_{\mathrm{G}}(\text { left-child })-i_{\mathrm{G}}(\text { right-child })
$$

where $i_{G}$ (parent) means Gini index of a node before splitting (parent node), and $i_{G}$ (leftchild, or right-child) means the Gini index of the node after splitting from parent node.

The maximally constructed decision tree can have some statistically insignificant nodes which leads to be the separation performance worse (overtraining). "Pruning" method is used to avoid the overtrainig as possible. "Cost-complexity pruning" is used in this search. The cost-complexity in a tree $T$ starting at node $t$ is expressed by

$$
R_{\alpha}\left(T_{t}\right)=R\left(T_{t}\right)+\alpha \cdot N\left(T_{t}\right)
$$

where, $R\left(T_{t}\right)$ is the total error cost in the tree $T$, the error cost in each terminal node is given by multiplying the $1-\max (p, 1-p)$ by the proportion of data, $\alpha$ is the cost complexity parameter, and $N\left(T_{t}\right)$ is the number of terminal nodes in the tree $T$, while the cost-complexity at node $t$ is

$$
R_{\alpha}(t)=R(t)+\alpha
$$

As long as $R_{\alpha}(t)>R_{\alpha}\left(T_{t}\right)$ the tree $T$ has a smaller cost-complexity than the single node $t$, in other words, it is worth to keep this node expanded. The inequality also expressed as the follows,

$$
\alpha<\frac{R(t)-R\left(T_{t}\right)}{N\left(T_{t}\right)-1}
$$

The node $t$ with the $\alpha$ in the tree $T$ is recursively pruned away as long as violating (5.6). Overtraing is managed by using the pruning method to some extent.

\subsubsection{Boosting Algorithm}

As described before, a response from a single decision tree has instability due to statistical fluctuation in the training samples. To resolve the problem, the boosting method is applied to one single decision tree. At first, the weight for each event in training samples is set to uniform, that is, no weight. After making a decision tree, we can see that some events are misclassified. Boosting makes the misclassified events weighted, 
and the next new decision tree is constructed using the weighted samples. This procedure is repeated by the number of trees we set. Then, many trees are built up. The score from the $m$ th individual tree $T_{m}$ is taken as +1 if the events falls on a signal leaf and -1 if the event falls on a background leaf. The final score is taken as a weighted sum of the scores of the individual leaves. In the following, "Adaboost" algorithm is explained, which is one of the boosting algorithm and used in this analysis.

Suppose that there are $N$ events in the samples. The events are assigned the weight $1 / N$ at first. Some notations are defined as follows:

- $x_{i}$ is the set of information (for example $p_{T}$ or $E_{T}$ ) for the $i$ th event,

- $y_{i}=1$ if the $i$ th event is a signal event and $y_{i}=-1$ if the $i$ th event is a background event,

- $w_{i}$ is the weight of the $i$ th event,

- $T_{m}\left(x_{i}\right)=1$ if the set of information for the $i$ th event lands that event on a signal leaf and $T_{m}\left(x_{i}\right)=-1$ if the set of information for that event lands it on a background leaf,

- $I\left(y_{i} \neq T_{m}\left(x_{i}\right)\right)=1$ and $I\left(y_{i}=T_{m}\left(x_{i}\right)\right)=0$,

where $m$ is index for $M$-th tree. Using the above notations, define the misclassification rate error,

$$
e r r_{m}=\frac{\sum_{i=1}^{N} w_{i} I\left(y_{i} \neq T_{m}\left(x_{i}\right)\right)}{\sum_{i=1}^{N} w_{i}}
$$

The error is used to change the weight of each event

$$
\begin{gathered}
\alpha_{m}=\beta \times \ln \left(\frac{1-e r r_{m}}{e r r_{m}}\right) \\
w_{i} \rightarrow w_{i} \times e^{\alpha_{m} I\left(y_{i} \neq T_{m}\left(x_{i}\right)\right)} .
\end{gathered}
$$

where $\beta=1$ is the standard AdaBoost method. The changed weights are normalized to

$$
w_{i} \rightarrow \frac{w_{i}}{\sum_{i=1}^{N} w_{i}} .
$$

The score for a given event is

$$
T(x)=\sum_{m=1}^{M} \alpha_{m} T_{m}(x)
$$


which is the weighted sum of the scores of the individual trees. The boosting algorithm remedies the statistical fluctuation in the samples and improves the separation performance between signal and background. The BDT framework is implemented in TMVA package [67] integrated in ROOT framework [66], which is used in this search.

\subsubsection{BDT Training Samples}

From the background estimation in section 4, there are two main background events for like-sign dilepton events. One is residual-photon conversions event, which is one electron originated from photon conversion with unobserved partner track. The other is fake lepton events. The components of the fake-lepton are interactive $\pi^{ \pm}$, overlap of $\pi^{0}$ and a track, punch-through hadrons, and non-prompt leptons. The BDT discriminant is optimized to well separate the events of the Higgs boson from the two main backgrounds. The procedure is called "training". The training samples for the signal are Higgs MC samples based on the theoretical properties described in Table 5.1, while samples for the backgrounds are residual-photon conversion and fake lepton events, which are derived mainly from the data. The training is performed using a sample on each mass of the Higgs boson with the main two backgrounds. These samples are passing LS-dilepton selection criteria.

\subsubsection{BDT Input Variables}

The BDT is insensitive to the input variables which have low separation powers compared with other multivariate tequniques, e.g. Artificial Neural Network, because the pruning procedure can remove the insignificant terminal nodes under such variables. However, if strongly correlated variables are selected, the performance should not be always good. We have selected 10 variables to be used as input variables into BDT:

- 1st lepton $p_{T}\left(p_{T 1}\right)$

- 2nd lepton $p_{T}\left(p_{T 2}\right)$

- Dilepton system $p_{T}\left(p_{T 12}\right)$

- Missing $E_{T}$

- Dilepton mass

- MetSpec : $\not_{T}$ if $\Delta \phi\left(\not_{T}, \ell\right.$ or jet $)>\pi / 2$ or

$$
: \mathbb{E}_{T} \sin \left(\Delta \phi\left(\mathbb{E}_{T}, \ell \text { or jet }\right)\right) \text { if } \Delta \phi\left(E_{T}, \ell \text { or jet }\right)<\pi / 2
$$

- $\mathrm{H}_{T}$ (Sum of $p_{T 1}, p_{T 2}$, jets $E_{T}\left(E_{T}>15 \mathrm{GeV}\right.$ and $\left.|\eta|<2.0\right)$, and Missing $\left.E_{T}\right)$

- Number of Jets with $E_{T}>15.0 \mathrm{GeV}$

- Sphericity 
- Aplanarity.

The sphericity tensor is defined as

$$
S^{\alpha \beta}=\frac{\sum_{i} p_{i}^{\alpha} p_{o}^{\beta}}{\sum_{i}\left|\mathbf{p}_{i}\right|^{2}},
$$

where $\alpha, \beta=1,2,3$ corresponds to the $x, y$, and $z$ components, and $p_{i}$ is the 4momentum for the $i$-th particle in the event. Generally, sphericity tensor has three eigenvalues, $\lambda_{1}, \lambda_{2}$, and $\lambda_{3}$ with the constraint $\lambda_{1} \geq \lambda_{2} \geq \lambda_{3}$ and $\lambda_{1}+\lambda_{2}+\lambda_{3}=1$. Then, sphericity $S$ is defined by

$$
S=\frac{3}{2}\left(\lambda_{2}+\lambda_{3}\right)
$$

so that $0 \leq S \leq 1$. The Aplanarity $A$ is defined by $A=3 / 2 \cdot \lambda_{3}$ and constrained to $0 \leq A \leq 1 / 2$.

The input variables are basically selected by the ranking of the BDT. A ranking of the BDT input variables is derived counting how often the variables are used to split decision tree nodes, and by weighting each split occurence by the separation gainsquared it has achieved and by the number of events in the node [64].

Figures 5.2 through 5.5 show 1 st lepton $E_{T}$, dilepton system $p_{z}$, and input variables in our like-sign dilepton signal region. We see no significant discrepancies between the data and the expected background events. 

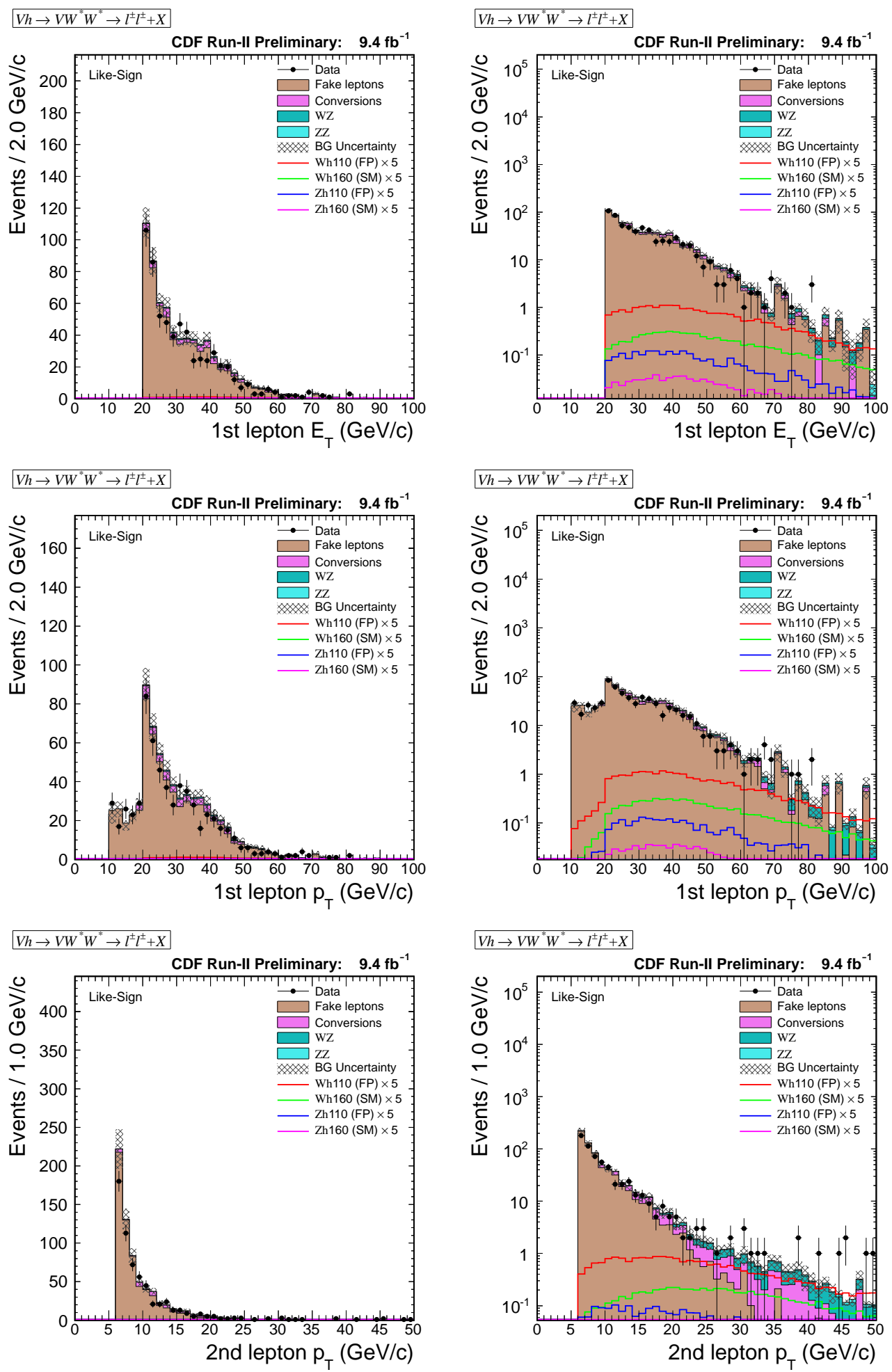

Figure 5.2: 1st lepton $E_{T}$, 1st lepton $p_{T}$, and 2nd lepton $p_{T}$ distributions in the signal region (left: linear, right: log). 

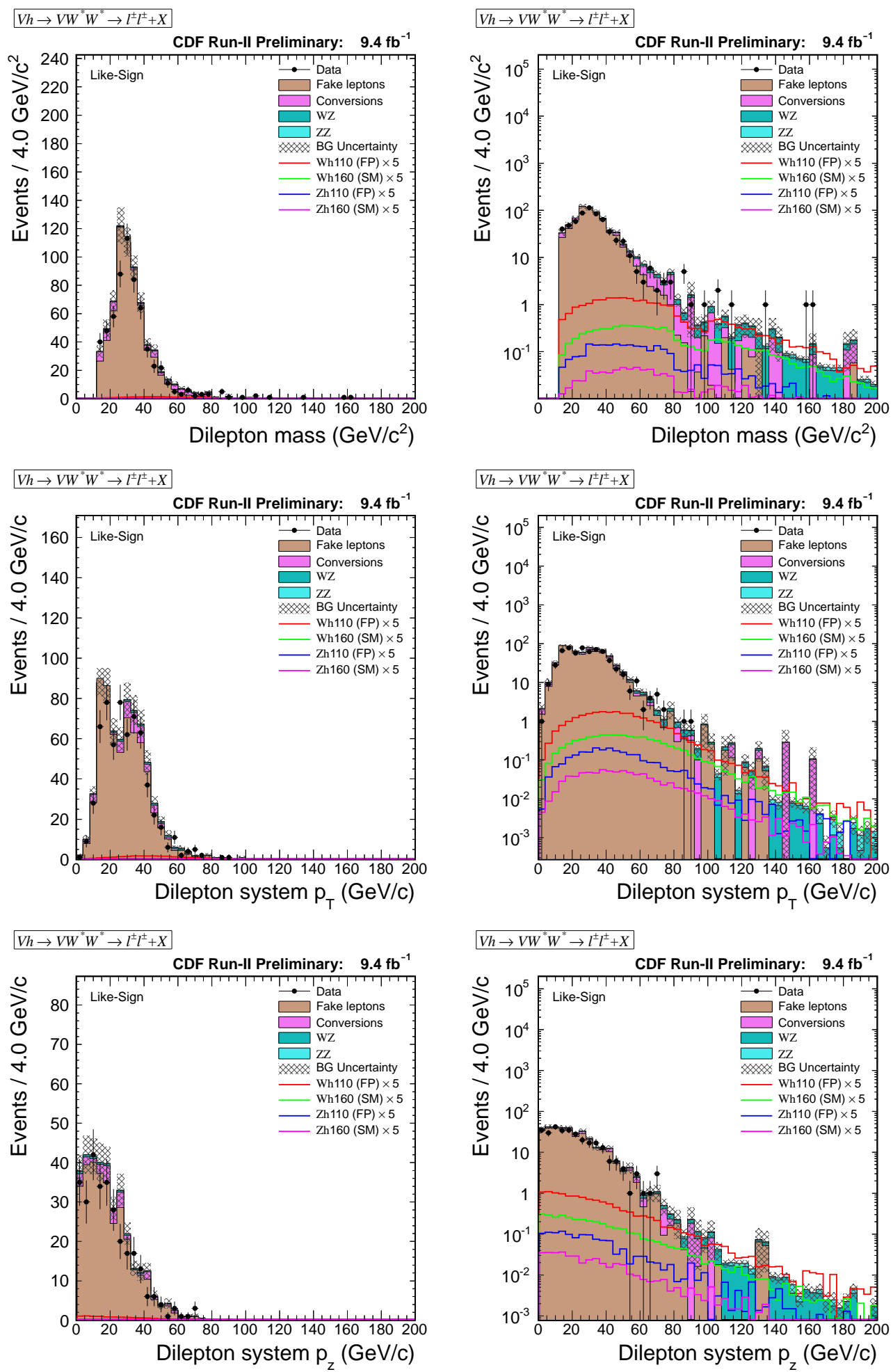

Figure 5.3: Dilepton mass, dilepton system $p_{T}$, and dilepton system $p_{z}$ in the signal region (left: linear, right: log). 

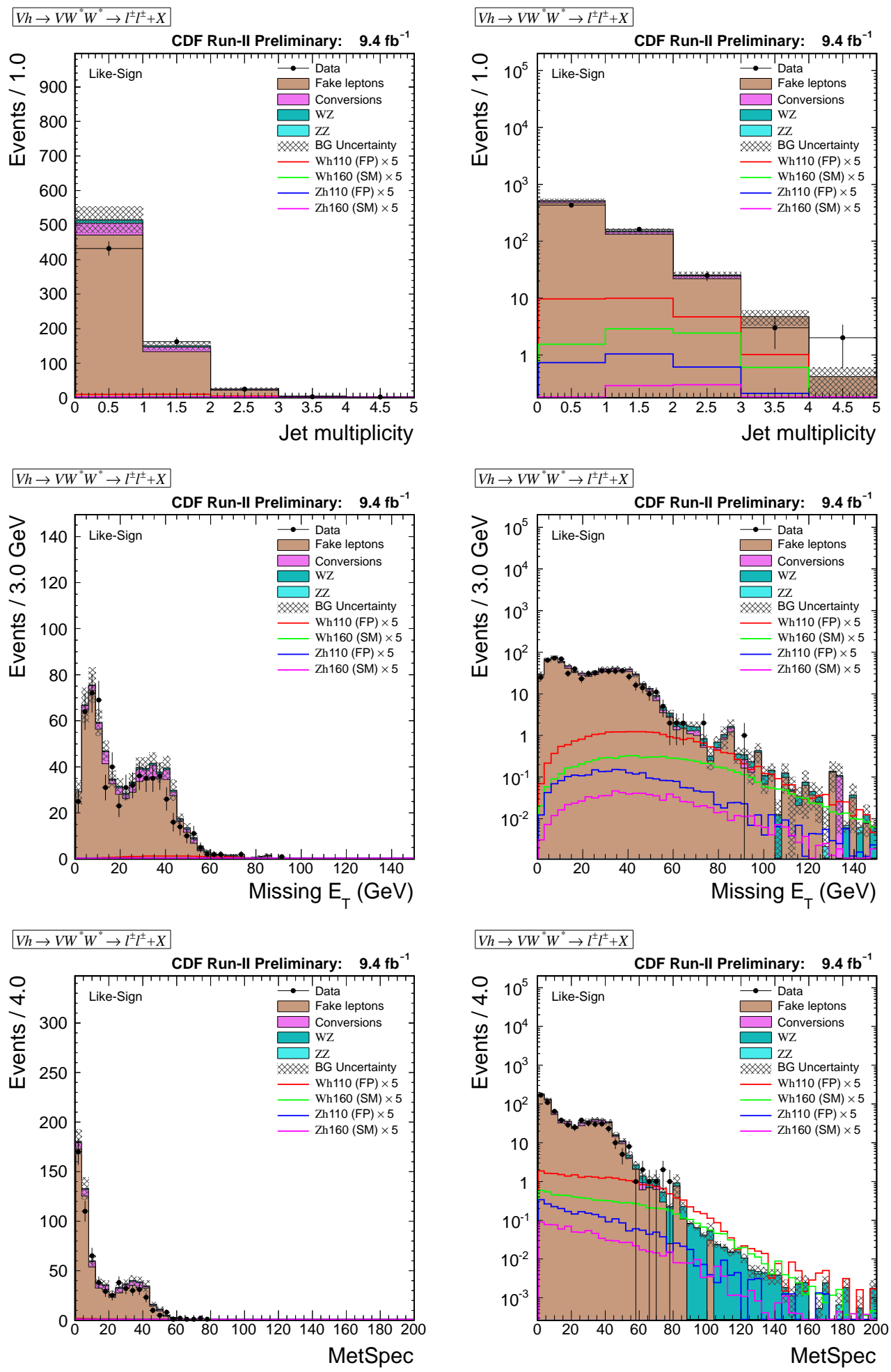

Figure 5.4: Jet multiplicity, missing $E_{T}$, and MetSpec in the signal region (left: linear, right: $\log$ ). 

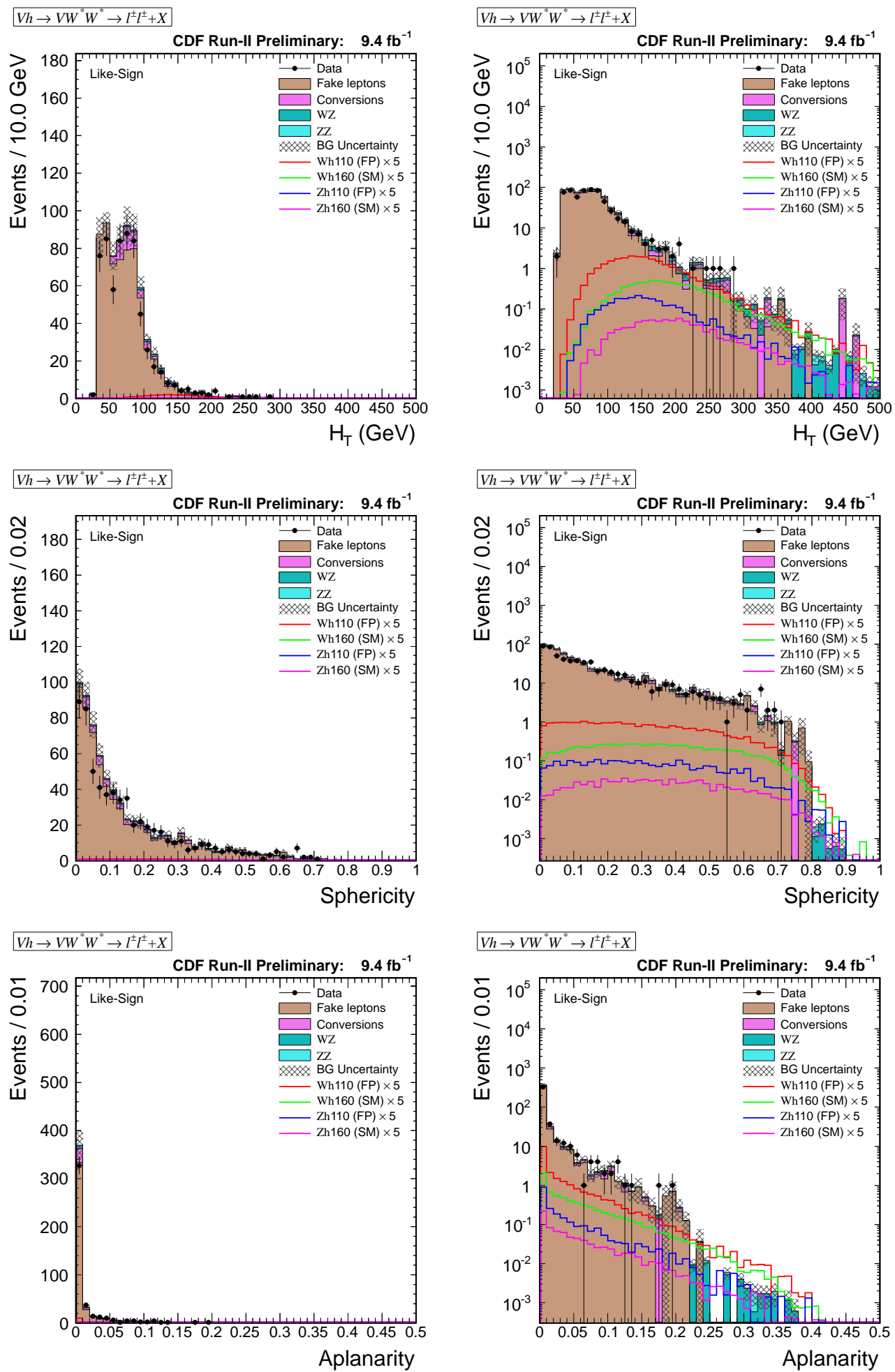

Figure 5.5: $H_{T}$, Sphericity, and Aplanarity in the signal region (left: linear, right: $\log$ ). 


\subsubsection{BDT Output and Cross Check}

The BDT output is used to discriminate Higgs events from backgrounds. The trained BDT is validated using three pilot regions: lepton-ID side-band, OS dilepton, and Zero-silicon event. These regions are introduced in $\S 4.4$ when we have checked the consistency between the expected number of events and data. For convenience, we shall introduce the regions again here. The Lepton-ID side-band is defined by

- LS dilepton passing lepton selection cuts, but

- The 2nd lepton is failed our lepton ID cuts.

Therefore, the region is dominated by fake-lepton events and used to validate the fakelepton estimation. The OS dilepton events is passing lepton selection and dilepton selection but OS combination not LS combination. The dominant process is from $Z / \gamma^{*}$ and estimated using MCs. Therefore, we use this pilot region as the validation of MC-based background expectation. The Zero-silicon region is defined as,

- LS dilepton passing lepton selection cuts, but

- The 1st lepton is not applied the silicon hit requirement

- The 2nd lepton is applied the zero-silicon hit requirement

In this region, events are dominantly residual-photon conversion events, to check the residual-photon conversion event expectation.

Figures 5.6 through 5.17 show the distributions in each pilot region, and we can see the backgrounds are well modeled and consistent with the data.

After looking background yield and the kinematic shape, the samples in three pilot regions are applied the trained BDT which is trained for LS-dilepton events. Figure 5.18, 5.19 and 5.20 show the BDT output in each three pilot regions on the mass of 110 and $160 \mathrm{GeV} / c^{2}$ of the Higgs boson. We can see reasonable agreement between the data distribution and expected events.

After confirming these results, we apply the trained BDT to the LS dilepton events. Figure 5.21 shows the BDT output for LS dilepton events on the Higgs mass 110 and $160 \mathrm{GeV} / c^{2}$, respectively. 

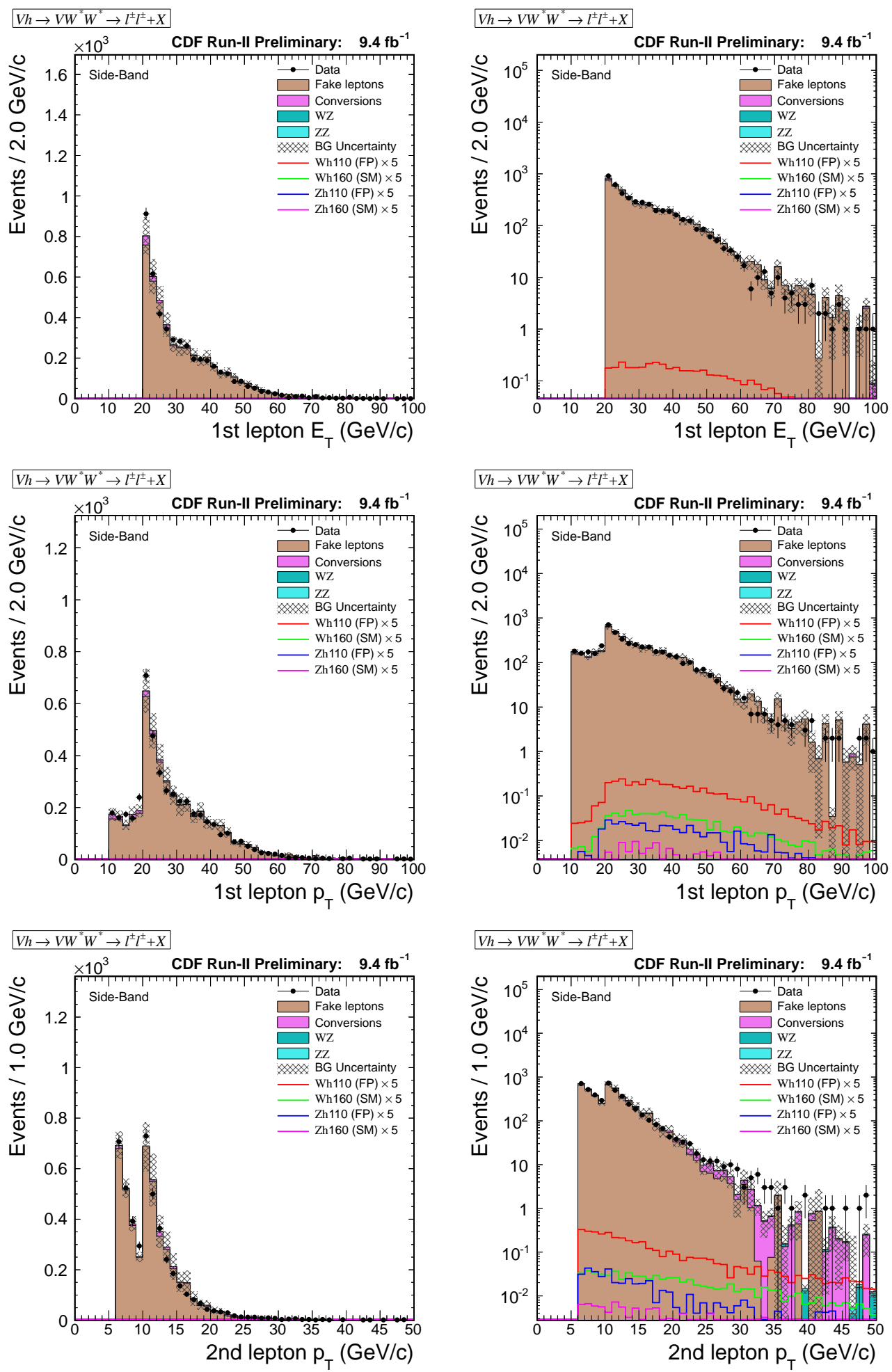

Figure 5.6: 1st lepton $E_{T}$, 1st lepton $p_{T}$, and 2nd lepton $p_{T}$ distributions in the lepton ID side-band region (left: linear, right: $\log$ ). 

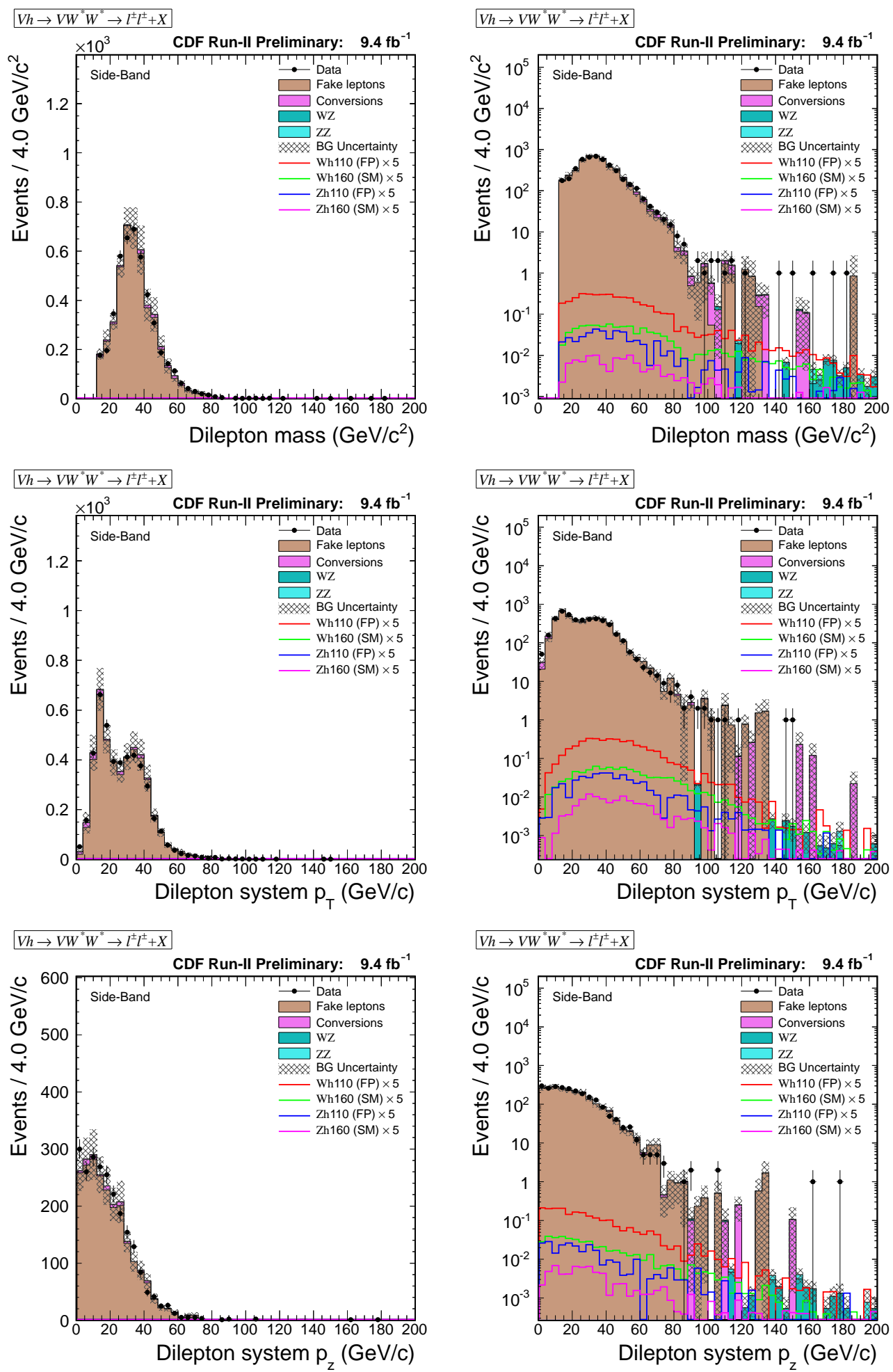

Figure 5.7: Dilepton mass, dilepton system $p_{T}$, and dilepton system $p_{z}$ in the lepton ID side-band region (left: linear, right: $\log$ ). 

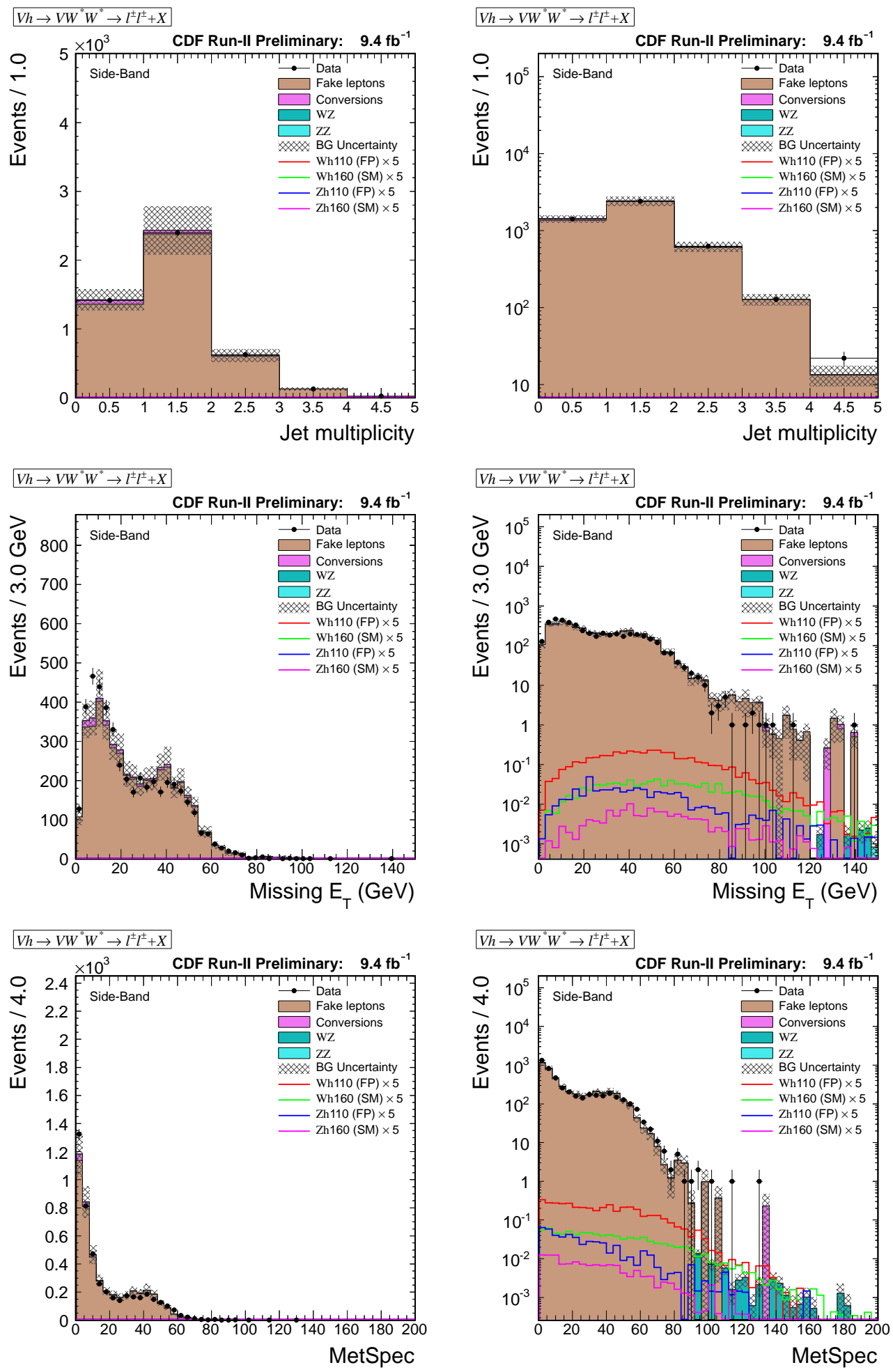

Figure 5.8: Jet multiplicity, missing $E_{T}$, and MetSpec in the lepton ID side-band region (left: linear, right: $\log$ ). 

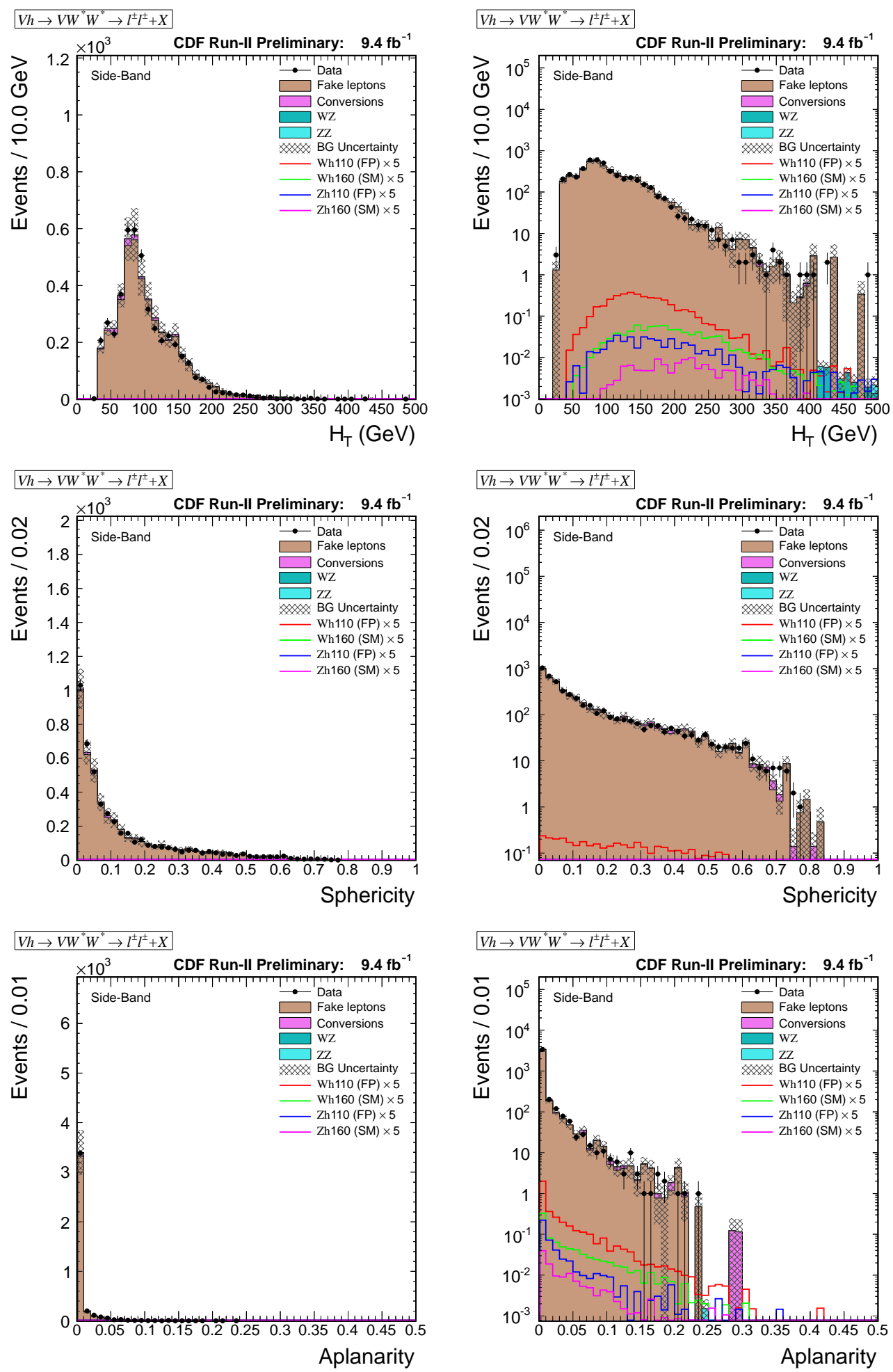

Figure 5.9: $H_{T}$, Sphericity, and Aplanarity in the lepton ID side-band region (left: linear, right: $\log$ ). 

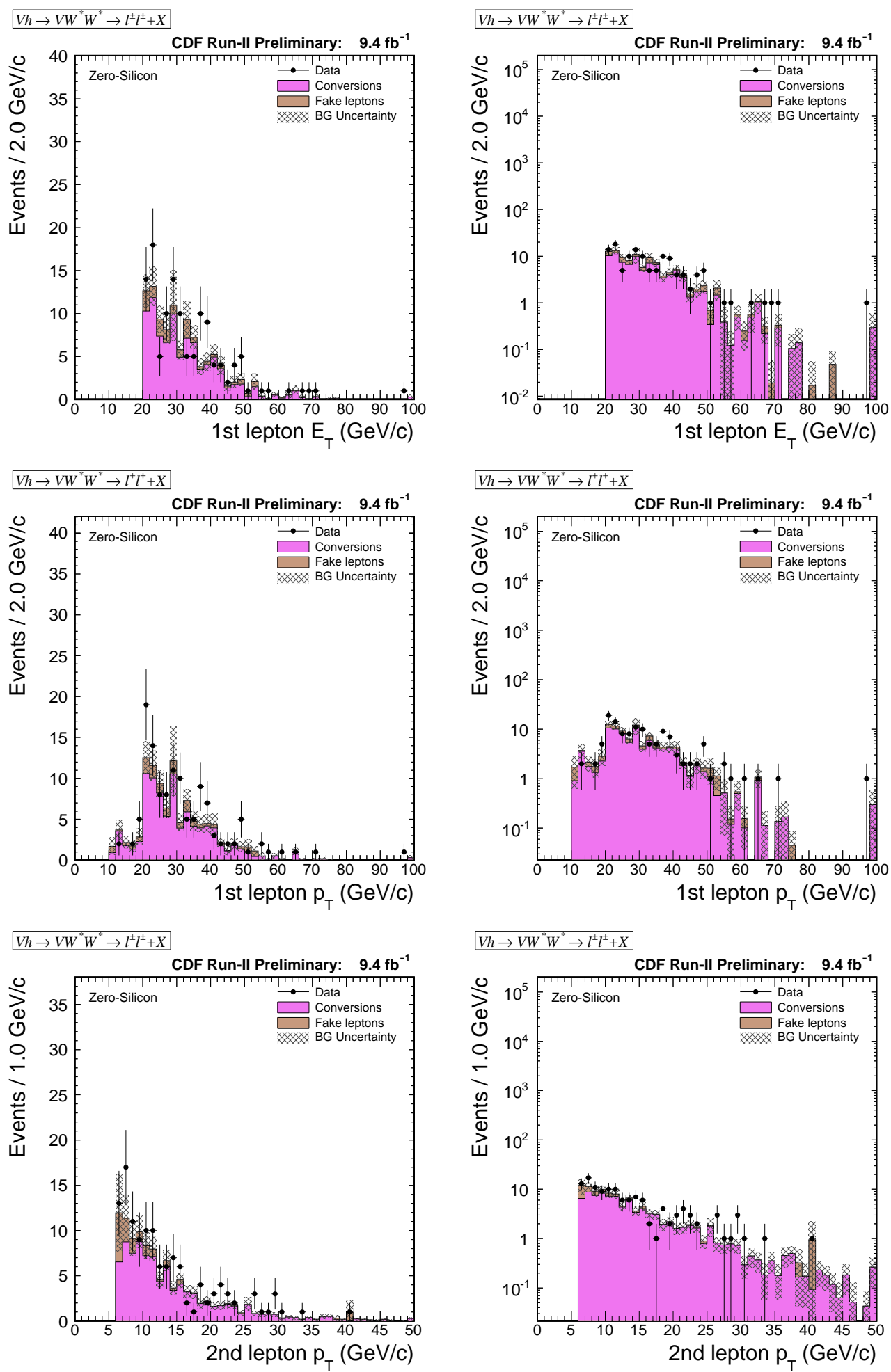

Figure 5.10: 1st lepton $E_{T}$, 1st lepton $p_{T}$, and 2nd lepton $p_{T}$ distributions in the zero-silicon region (left: linear, right: $\log$ ). 

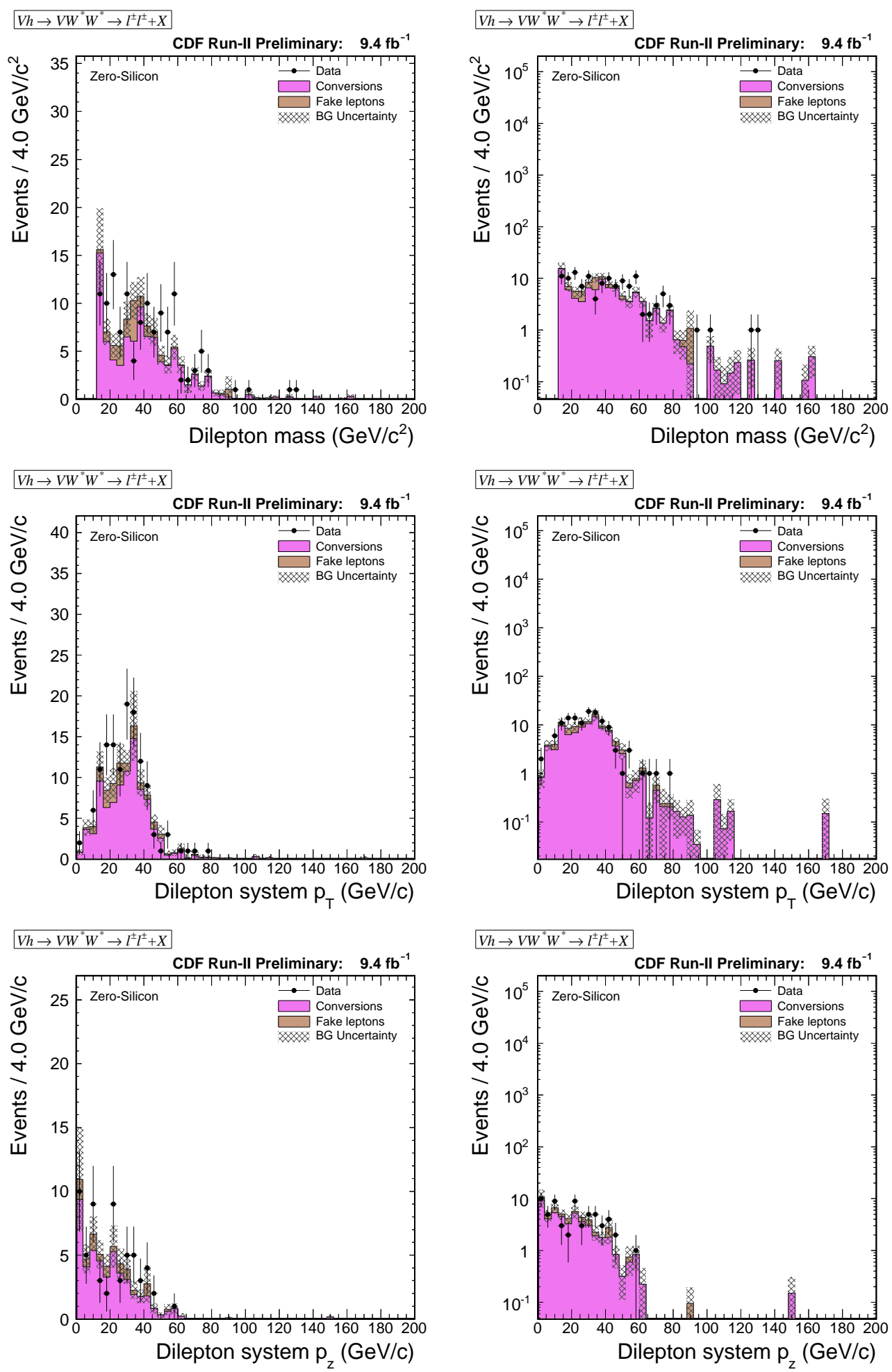

Figure 5.11: Dilepton mass, dilepton system $p_{T}$, and dilepton system $p_{z}$ in the zerosilicon region (left: linear, right: $\log$ ). 

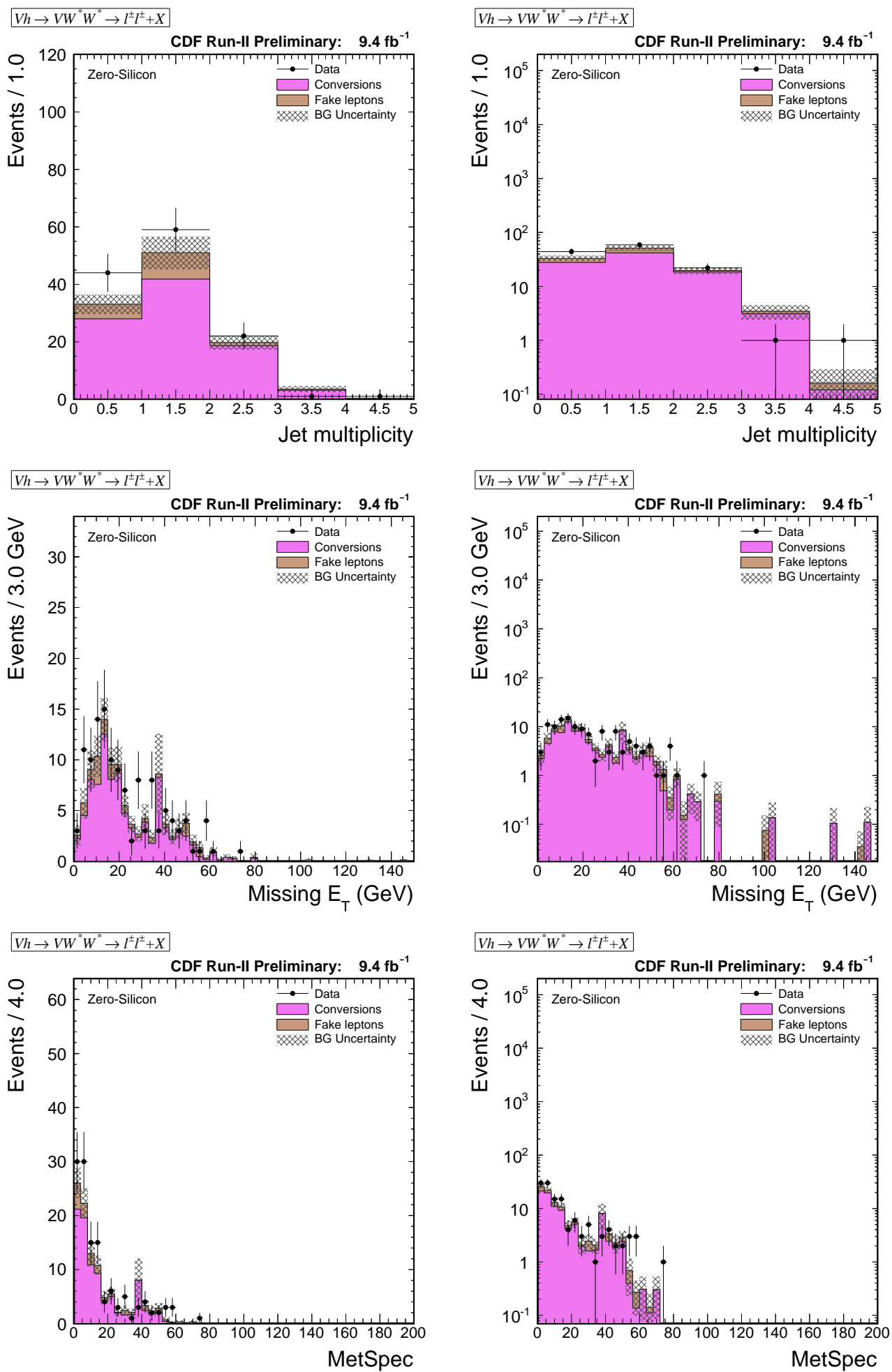

Figure 5.12: Jet multiplicity, missing $E_{T}$, and MetSpec in the zero-silicon region (left: linear, right: $\log )$. 

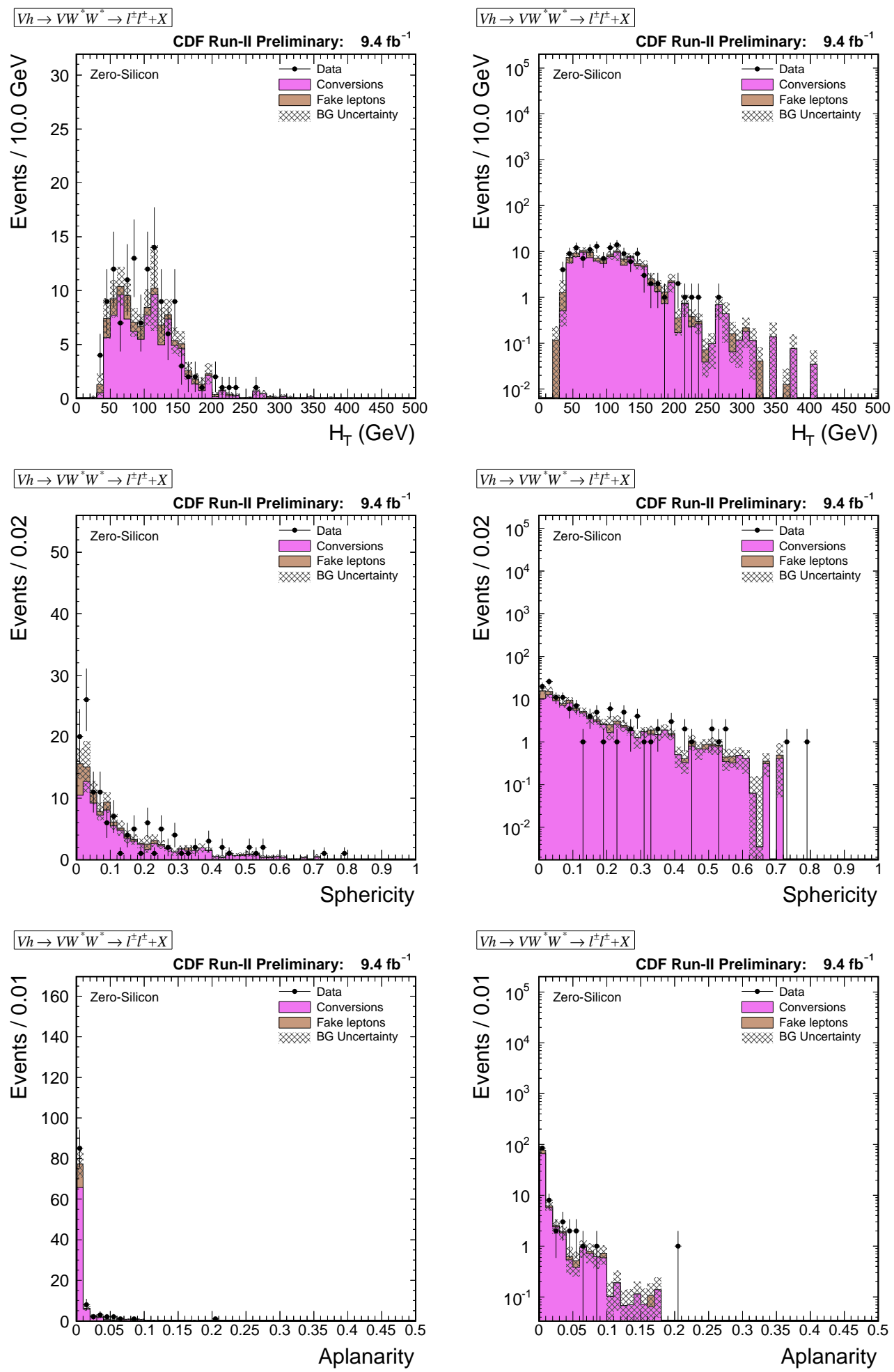

Figure 5.13: $H_{T}$, Sphericity, and Aplanarity in the zero-silicon region (left: linear, right: $\log )$. 

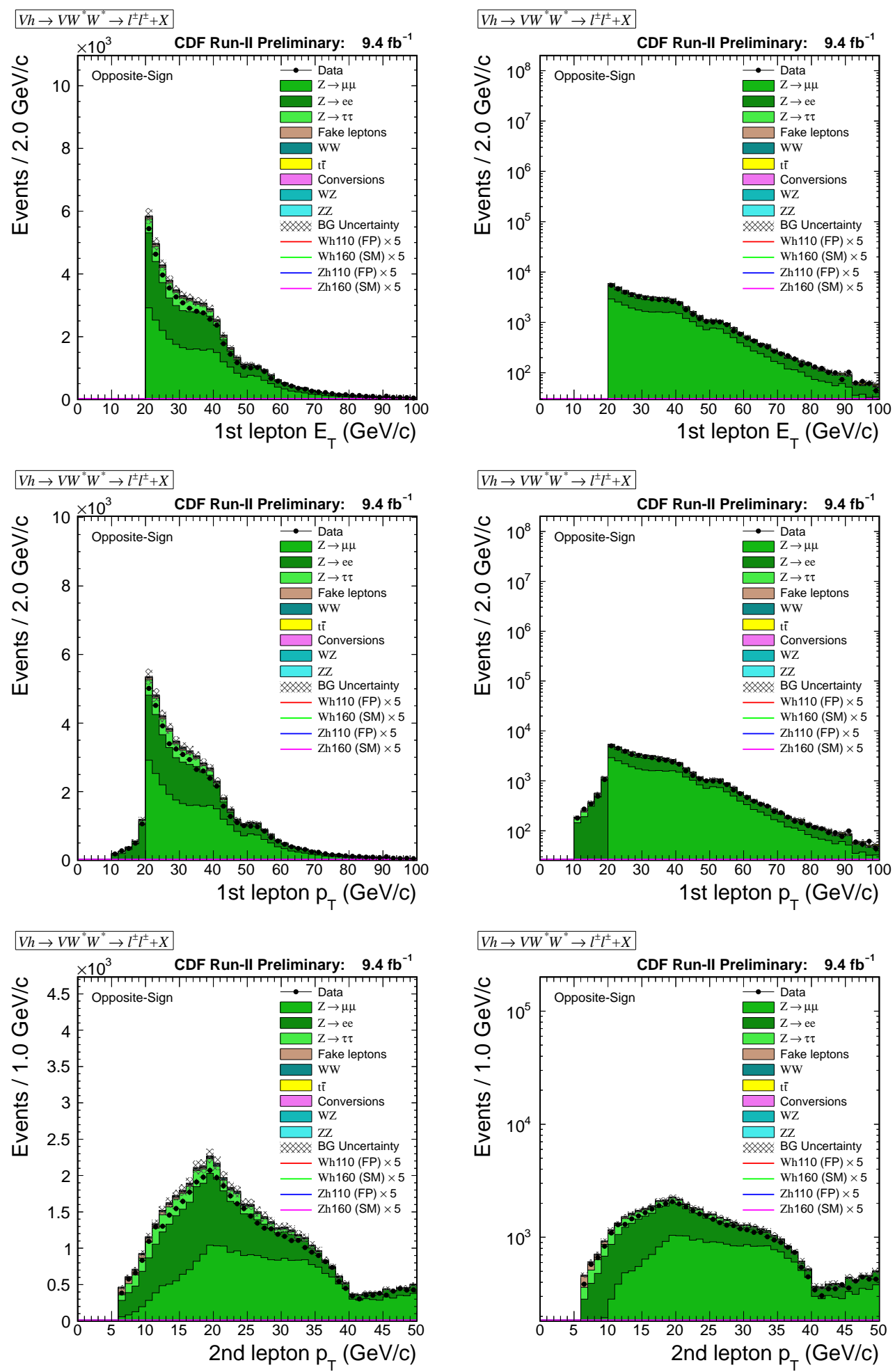

Figure 5.14: 1st lepton $E_{T}$, 1st lepton $p_{T}$, and 2nd lepton $p_{T}$ distributions in the opposite-sign region (left: linear, right: $\log$ ). 

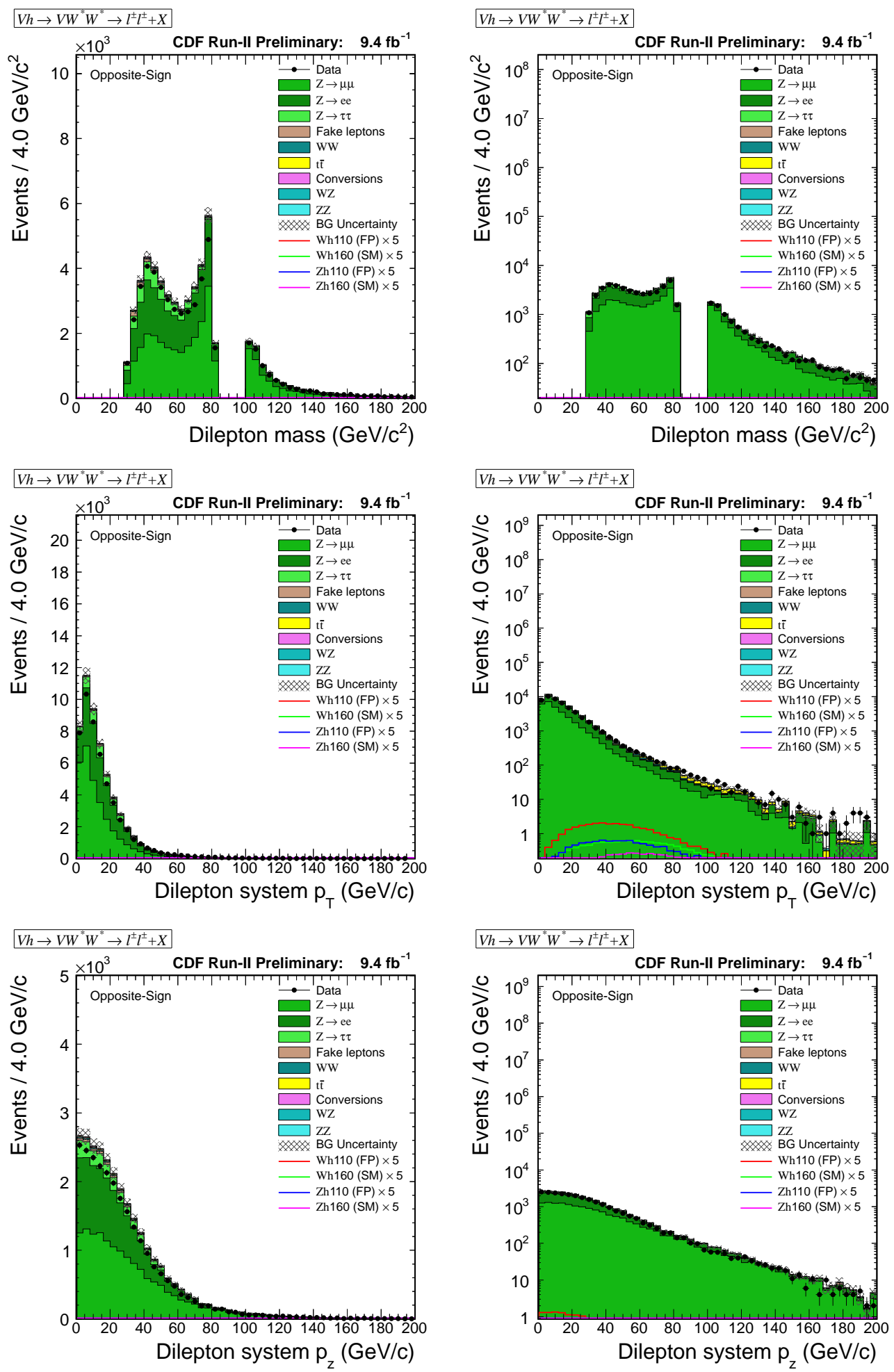

Figure 5.15: Dilepton mass, dilepton system $p_{T}$, and dilepton system $p_{z}$ in the oppositesign region (left: linear, right: log). 

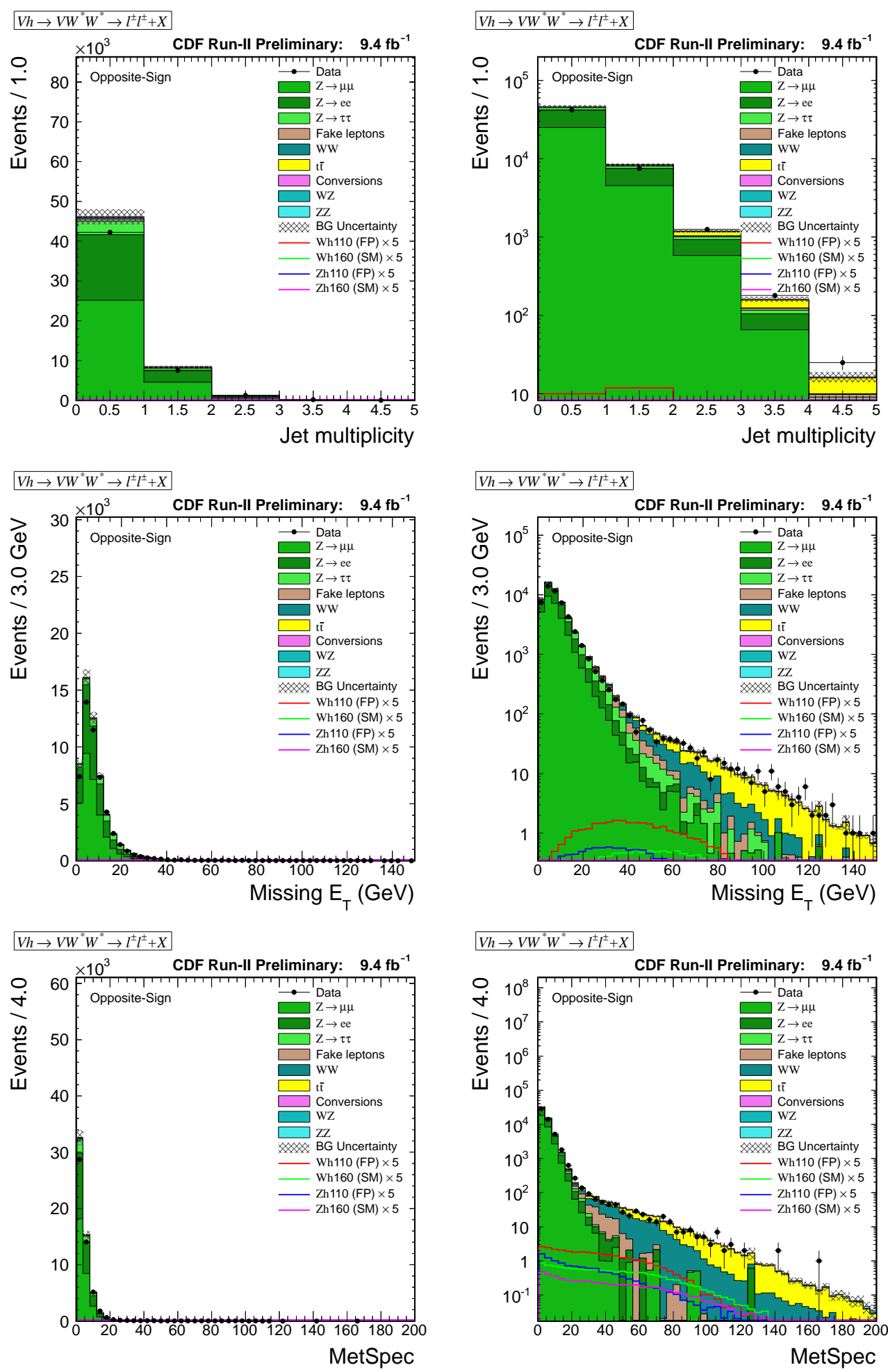

Figure 5.16: Jet multiplicity, missing $E_{T}$, and MetSpec in the opposite-sign region (left: linear, right: $\log$ ). 

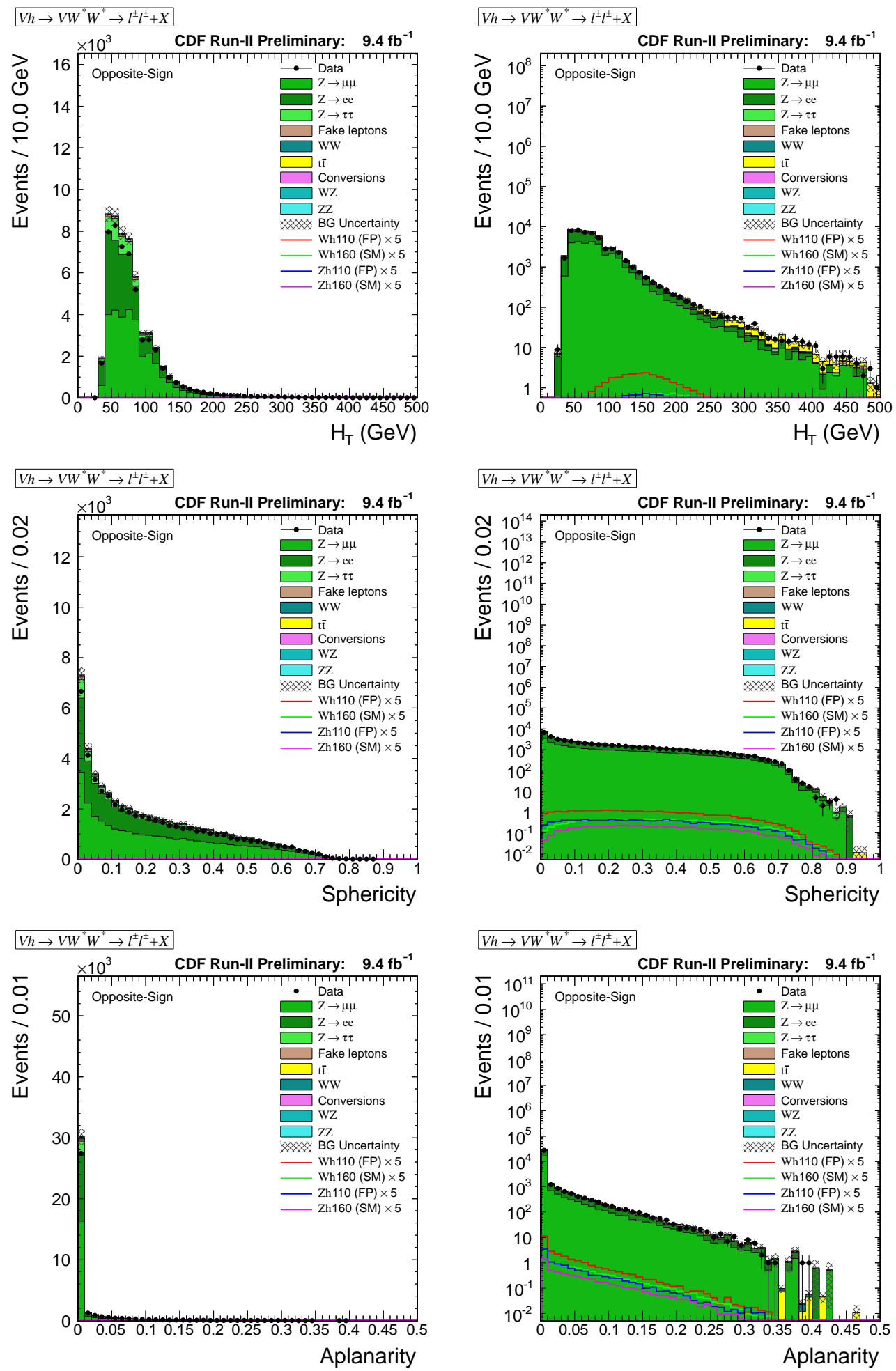

Figure 5.17: $H_{T}$, Sphericity, and Aplanarity in the opposite-sign region (left: linear, right: $\log )$. 

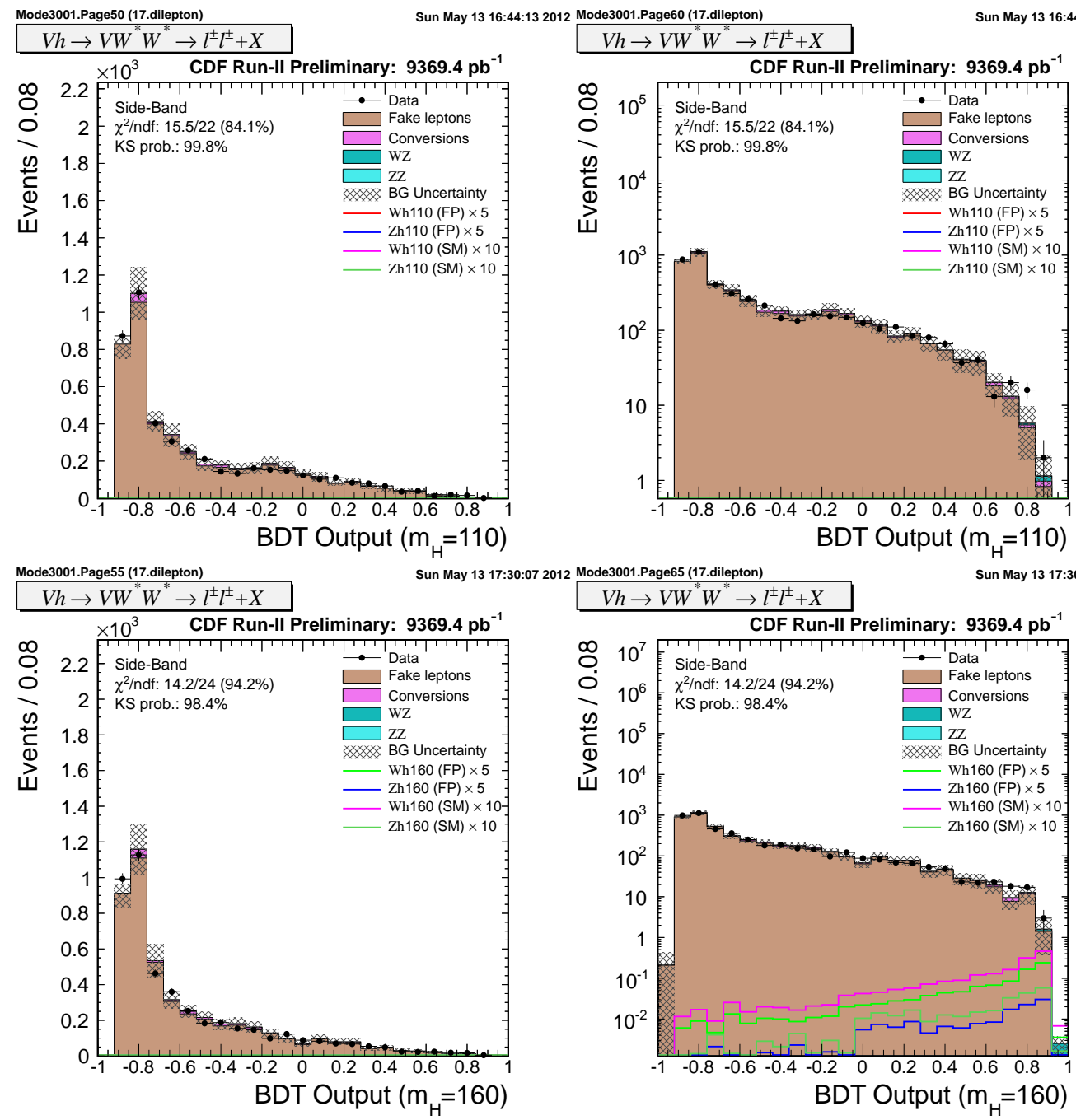

Figure 5.18: BDT output on $m_{H}=110,160\left(\mathrm{GeV} / c^{2}\right)$ in the side-band region (left: linear, right: $\log$ ). 

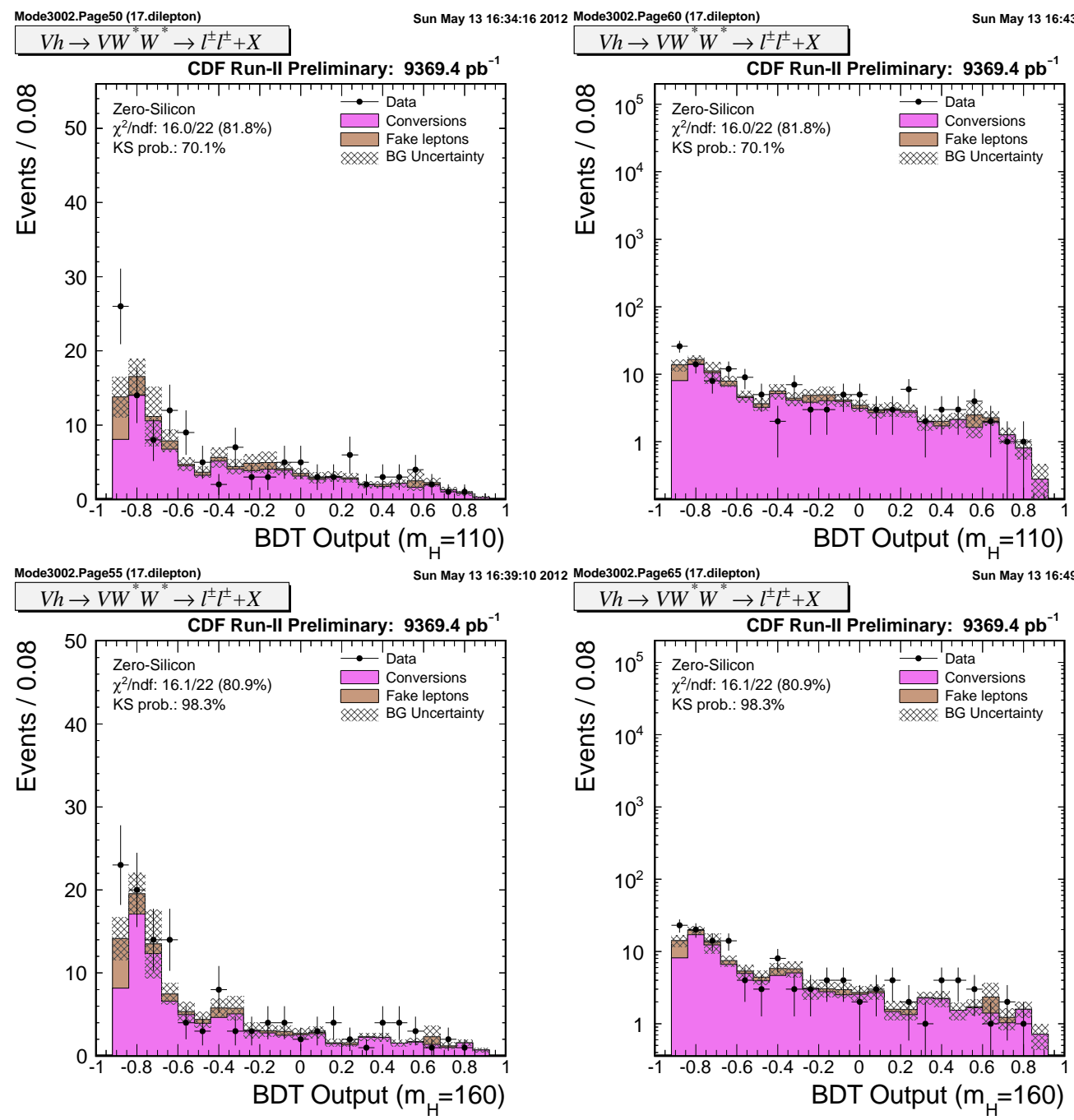

Figure 5.19: BDT output on $m_{H}=110,160\left(\mathrm{GeV} / c^{2}\right)$ in the zero-silicon region (left: linear, right: $\log$ ). 

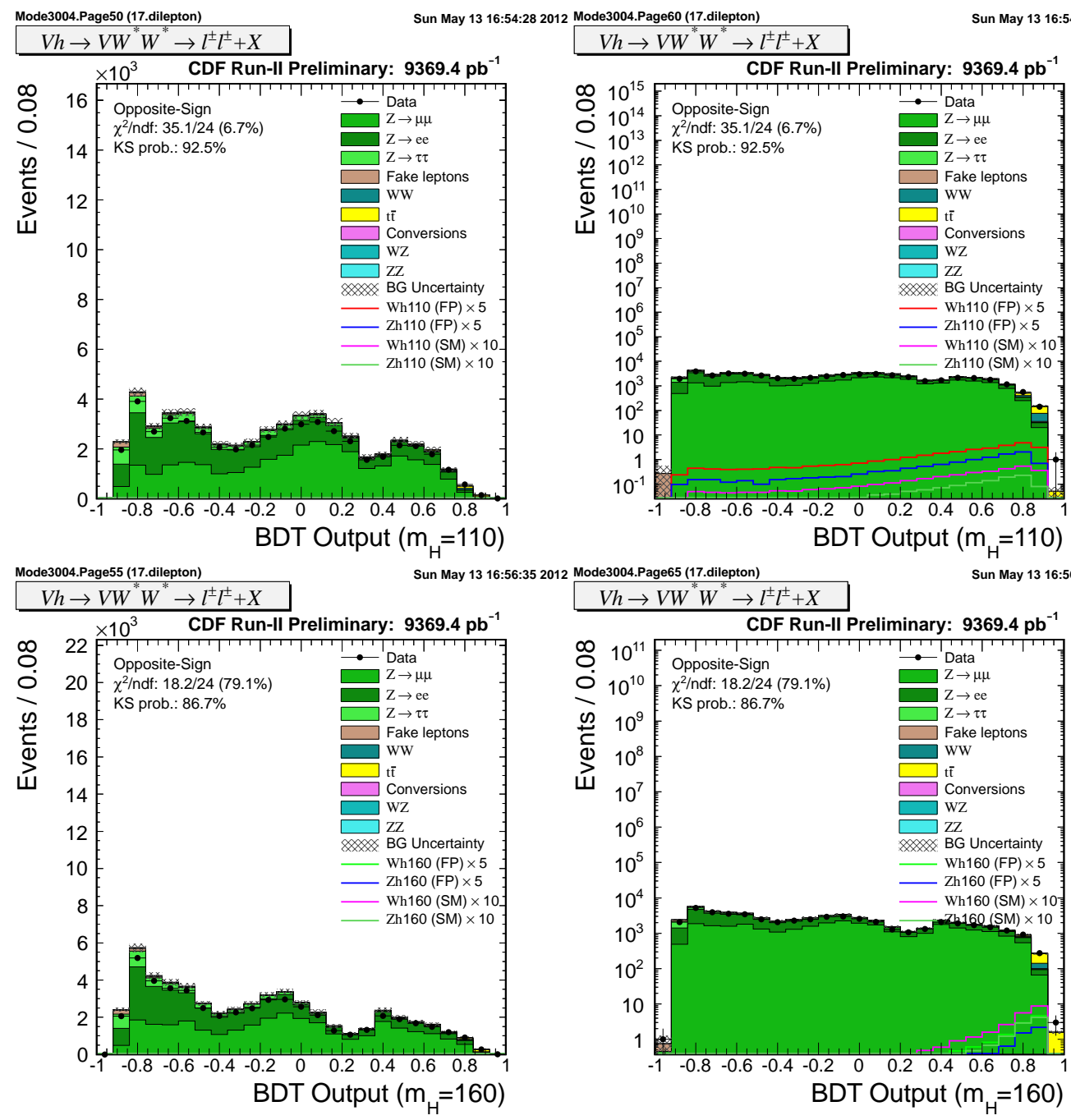

Figure 5.20: BDT output on $m_{H}=110,160\left(\mathrm{GeV} / c^{2}\right)$ in the OS region (left: linear, right: $\log$ ). 

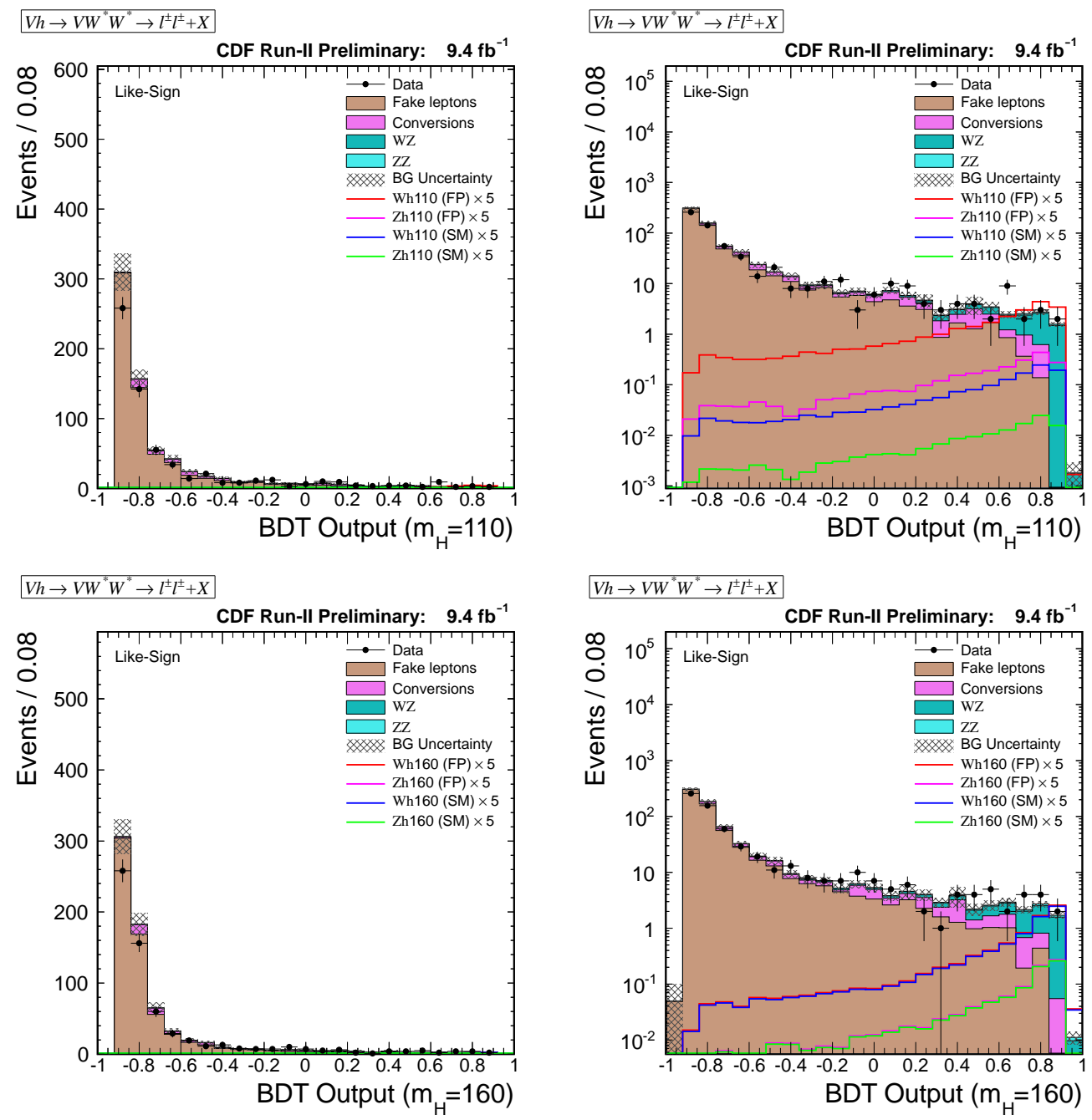

Figure 5.21: BDT output on $m_{H}=110,160\left(\mathrm{GeV} / c^{2}\right)$ in the signal region (left: linear, right: $\log$ ). 


\section{Chapter 6}

\section{Systematic Uncertainty}

There are the systematic uncertainties from several sources. The systematic uncertainties are classified into two types in this search. One is "Rate systematic uncertainty" which affects the event yield and acceptance, and the other is "Shape systematic uncertainty" which changes the shape to the relevant kinematics. Both systematic uncertainties strongly affects the search sensitivity. In this chapter, systematic uncertainties in the LS dilepton analysis are explained.

\subsection{Rate Systematic Uncertainty}

The rate systematic uncertainties affect the event yield (acceptance). The systematic uncertainties are taken into account for both the signal and background for several systematic sources.

- Parton Distribution Function (PDF):

A momentum of a parton in a proton is described by the PDF which is derived by measurements from several experiments and theoretical calculations. In CDF, Monte Carlo simulations use CTEQ5L as the default PDF set. The PDF uncertainty is estimated by calculating acceptance using other PDF set with taking into account the difference of them. The other PDF are 2 CTEQ6Ls with changing $\alpha_{s}$ value, 2 MRST set changing $\alpha_{s}$ and not, and 40 CTEQ6Ms changing eigenvector. The uncertainty is associated with the Higgs boson MC samples.

- Initial and Final State Radiation (ISR and FSR):

The gluon and photon radiation at initial and final state give correction to the event topology. The correction to the acceptance is estimated as systematic uncertainty using the MC samples changing the parameters related to the ISR and the FSR from default MC samples. The systematic uncertainties are estimated for the Higgs MC samples.

- Production cross section:

The several production cross sections are estimated by theoretical calculation 
taking experiment results. The uncertainty from the prediction calculation is concerned as systematic uncertain in acceptance. The uncertainty of Higgs prediction cross section $\sigma(V h \rightarrow p \bar{p})$ is noticed as $5 \%$ in [68]. For backgrounds, both $W W$ and $W Z$ uncertanities are mentioned in [69] as $10 \%$.

- $Z / \gamma^{*}$ cross section:

As described at $\S 4.3 .11, Z / \gamma^{*} \rightarrow \ell^{+} \ell^{-}$cross sections are estimated to validate the trigger efficiency and the scale factors for the primary vertex and lepton identification efficiencies. The disagreement between the data and the expectation is taken as the systematic uncertainty (4.6\%).

- Luminosity:

The luminosity measurement has been done by the CLC detector as described in $\S 2.7$ in CDF experiment. The measurement has several considerable systematic uncertainties, that is, the uncertainty of the inelastic $p \bar{p}$ cross section and CLC acceptance. The systematic uncertainties from the measurement is total $6 \%$, which is for MC samples.

- Residual photon-conversion ratio and fake lepton rate:

The background expectations for fake lepton and residual photon-conversion are derived from the data with the weights, that is, fake lepton rate and residual photon-conversion ratio. The weight systematic uncertainty mainly comes from data/MC statistics and uncertainties between used samples, for example jet samples for fake lepton rate.

The summary of rate systematic uncertainties is shown in Tables 6.1 and 6.2 for signal (Higgs) and Table 6.3 for backgrounds.

Table 6.1: Systematic uncertainties for the $W h$ Monte Carlo samples.

\begin{tabular}{ccccccccccc}
\hline \hline Higgs Mass $\left(\mathrm{GeV} / c^{2}\right)$ & 110 & 120 & 130 & 140 & 150 & 160 & 170 & 180 & 190 & 200 \\
\hline Statistics & $0.8 \%$ & $0.8 \%$ & $0.8 \%$ & $0.7 \%$ & $0.7 \%$ & $0.7 \%$ & $0.7 \%$ & $0.7 \%$ & $0.7 \%$ & $0.7 \%$ \\
PDF & $2.2 \%$ & $1.9 \%$ & $1.6 \%$ & $1.6 \%$ & $1.4 \%$ & $1.2 \%$ & $1.4 \%$ & $1.1 \%$ & $0.8 \%$ & $0.7 \%$ \\
ISR & $4.0 \%$ & $4.0 \%$ & $4.0 \%$ & $4.0 \%$ & $4.0 \%$ & $4.0 \%$ & $4.0 \%$ & $4.0 \%$ & $4.0 \%$ & $4.0 \%$ \\
FSR & $5.3 \%$ & $5.3 \%$ & $5.3 \%$ & $5.3 \%$ & $5.3 \%$ & $5.3 \%$ & $5.3 \%$ & $5.3 \%$ & $5.3 \%$ & $5.3 \%$ \\
$Z / \gamma^{*}$ & $4.6 \%$ & $4.6 \%$ & $4.6 \%$ & $4.6 \%$ & $4.6 \%$ & $4.6 \%$ & $4.6 \%$ & $4.6 \%$ & $4.6 \%$ & $4.6 \%$ \\
Cross Section & $5.0 \%$ & $5.0 \%$ & $5.0 \%$ & $5.0 \%$ & $5.0 \%$ & $5.0 \%$ & $5.0 \%$ & $5.0 \%$ & $5.0 \%$ & $5.0 \%$ \\
Luminosity & $6.0 \%$ & $6.0 \%$ & $6.0 \%$ & $6.0 \%$ & $6.0 \%$ & $6.0 \%$ & $6.0 \%$ & $6.0 \%$ & $6.0 \%$ & $6.0 \%$ \\
\hline Total & $11.5 \%$ & $11.4 \%$ & $11.4 \%$ & $11.4 \%$ & $11.3 \%$ & $11.3 \%$ & $11.3 \%$ & $11.3 \%$ & $11.3 \%$ & $11.3 \%$ \\
\hline \hline
\end{tabular}


Table 6.2: Systematic uncertainties for the $Z h$ Monte Carlo samples.

\begin{tabular}{ccccccccccc}
\hline \hline Higgs Mass $\left(\mathrm{GeV} / c^{2}\right)$ & 110 & 120 & 130 & 140 & 150 & 160 & 170 & 180 & 190 & 200 \\
\hline Statistics & $2.3 \%$ & $2.2 \%$ & $2.1 \%$ & $2.1 \%$ & $2.0 \%$ & $2.1 \%$ & $2.1 \%$ & $2.1 \%$ & $1.9 \%$ & $1.9 \%$ \\
PDF & $2.5 \%$ & $2.0 \%$ & $1.5 \%$ & $1.8 \%$ & $1.8 \%$ & $1.3 \%$ & $1.3 \%$ & $1.0 \%$ & $1.1 \%$ & $0.4 \%$ \\
ISR & $4.0 \%$ & $4.0 \%$ & $4.0 \%$ & $4.0 \%$ & $4.0 \%$ & $4.0 \%$ & $4.0 \%$ & $4.0 \%$ & $4.0 \%$ & $4.0 \%$ \\
FSR & $5.3 \%$ & $5.3 \%$ & $5.3 \%$ & $5.3 \%$ & $5.3 \%$ & $5.3 \%$ & $5.3 \%$ & $5.3 \%$ & $5.3 \%$ & $5.3 \%$ \\
$Z / \gamma^{*}$ & $4.6 \%$ & $4.6 \%$ & $4.6 \%$ & $4.6 \%$ & $4.6 \%$ & $4.6 \%$ & $4.6 \%$ & $4.6 \%$ & $4.6 \%$ & $4.6 \%$ \\
Cross Section & $5.0 \%$ & $5.0 \%$ & $5.0 \%$ & $5.0 \%$ & $5.0 \%$ & $5.0 \%$ & $5.0 \%$ & $5.0 \%$ & $5.0 \%$ & $5.0 \%$ \\
Luminosity & $6.0 \%$ & $6.0 \%$ & $6.0 \%$ & $6.0 \%$ & $6.0 \%$ & $6.0 \%$ & $6.0 \%$ & $6.0 \%$ & $6.0 \%$ & $6.0 \%$ \\
\hline Total & $11.7 \%$ & $11.6 \%$ & $11.5 \%$ & $11.6 \%$ & $11.6 \%$ & $11.5 \%$ & $11.5 \%$ & $11.5 \%$ & $11.4 \%$ & $11.4 \%$ \\
\hline \hline
\end{tabular}

Table 6.3: Systematic uncertainties for backgrounds.

\begin{tabular}{ccccc}
\hline \hline & Fake leptons & Residual photon-conversion & $W Z$ & $Z Z$ \\
\hline Statistics & $2.1 \%$ & $11.1 \%$ & $0.7 \%$ & $2.1 \%$ \\
Fake rate & $7.9 \%$ & - & - & - \\
Residual conversion rate & - & $22.0 \%$ & - & - \\
$\mathrm{Z} / \gamma^{*}$ & - & - & $4.6 \%$ & $4.6 \%$ \\
Cross Section & - & - & $10.0 \%$ & $10.0 \%$ \\
Luminosity & - & - & $6.0 \%$ & $6.0 \%$ \\
\hline Total & $8.2 \%$ & $24.7 \%$ & $12.6 \%$ & $12.7 \%$ \\
\hline \hline
\end{tabular}

\subsection{Shape Systematic Uncertainty}

The shape systematic uncertainty affects relevant kinematic distributions, especially BDT input and output distributions. In this search, the shape uncertainties from jet energy correction need to be considered. Like-sign dilepton events need not specify jet objects, but in our case, some of jet information is used as the input variables into BDT shown in §5.2.4: for example, number of jets, missing $E_{T}$, MetSpec, and so on.

As described at $\S 3.2 .3$, the jet energy measured by calorimeters is corrected by using data and MCs for detector effects and physics effects. The shape systematic uncertainties are estimated by changing $\pm 1 \sigma$ from the default jet energy corrections. We do not set some cut related to the jet objects when looking at the like-sign dilepton events. Therefore, the rate systematic uncertainties related to the jet energy corrections are zero. The variations are shown in Figures 6.1 through 6.4. 

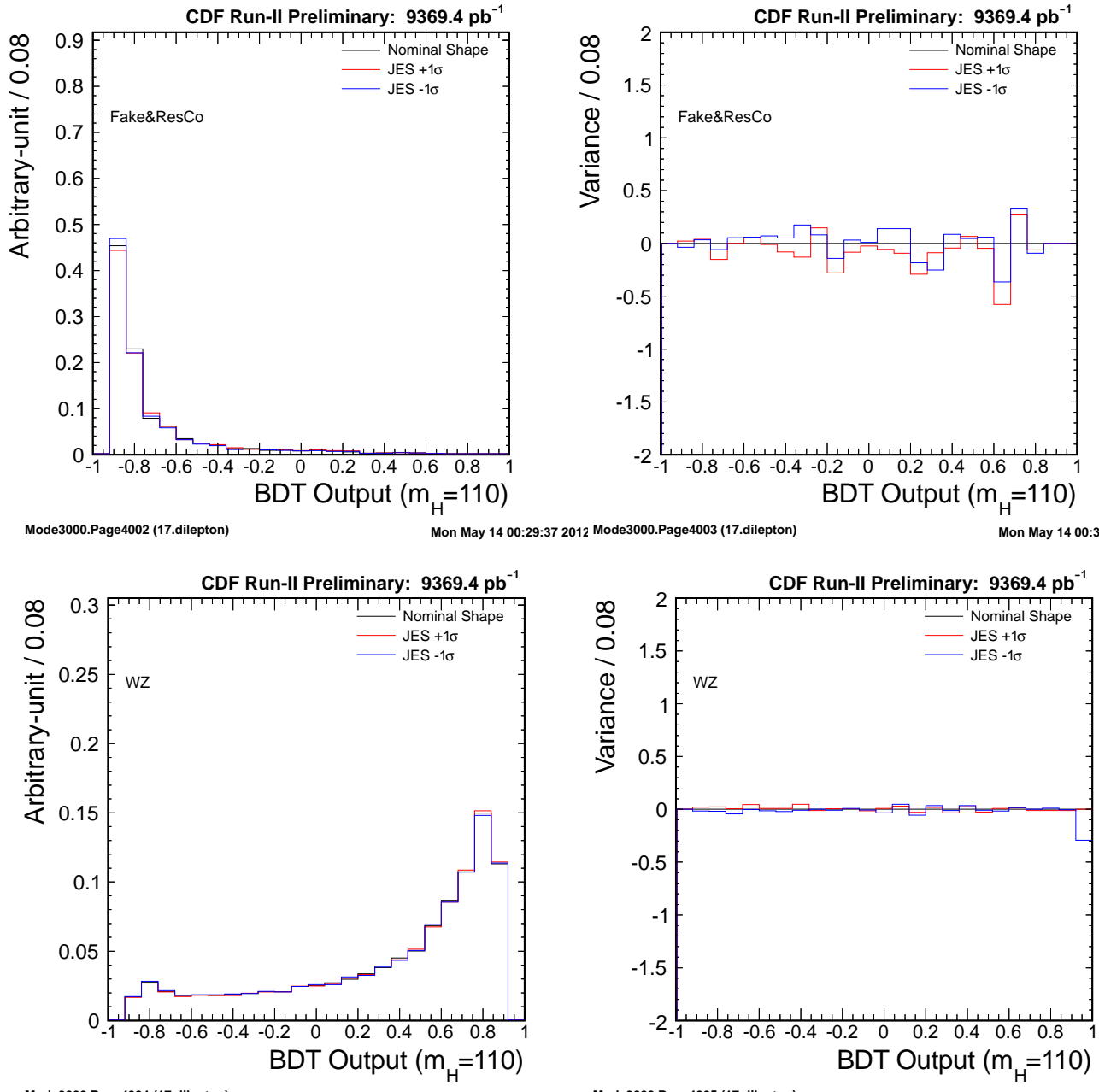

Mode3000.Page4004 (17.dilepton) Mon May 14 00:34:58 2012 Mode3000.Page4005 (17.dilepton)
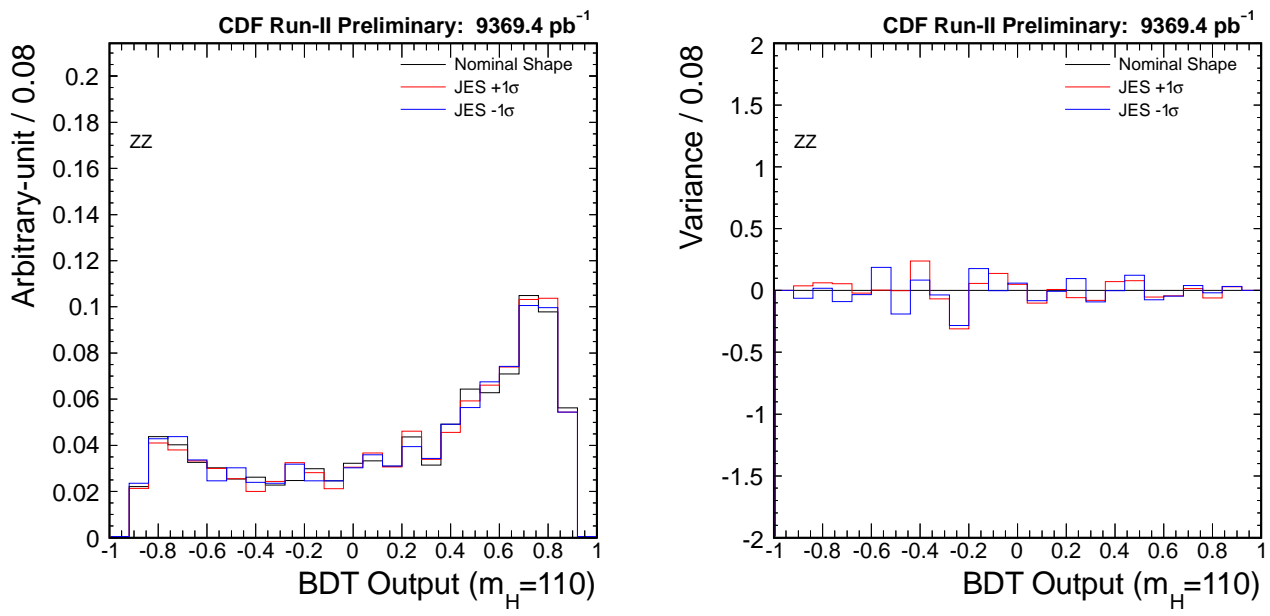

Figure 6.1: Shape systematic uncertainties due to jet energy scale for backgrounds trained on $m_{H}=110 \mathrm{GeV} / c^{2}$. (Fake\&ResCo means fake lepton and residual-photon conversion backgrounds.) 

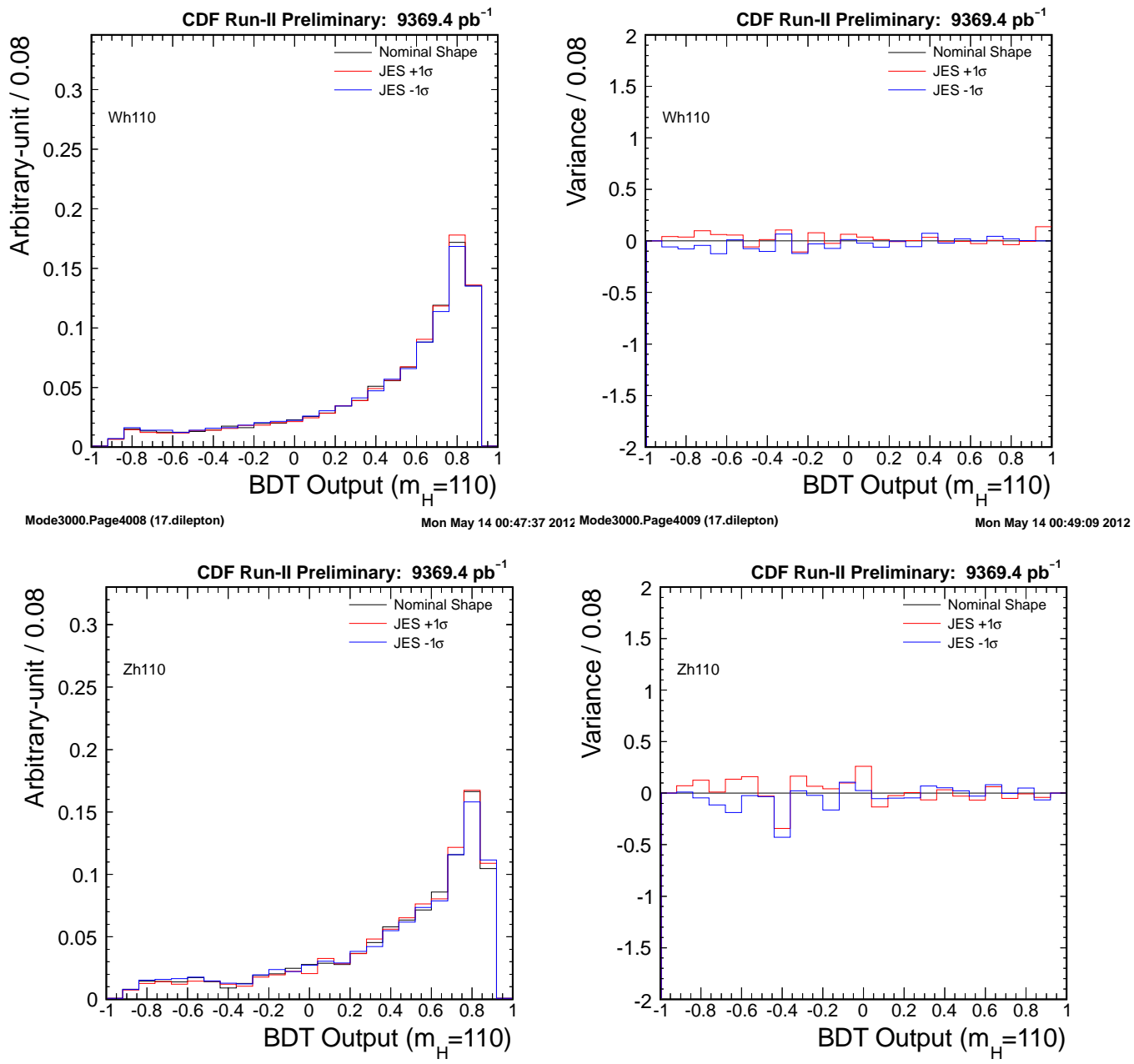

Figure 6.2: Shape systematic uncertainties due to jet energy scale for Higgs Mass 110 $\mathrm{GeV} / c^{2}$. 

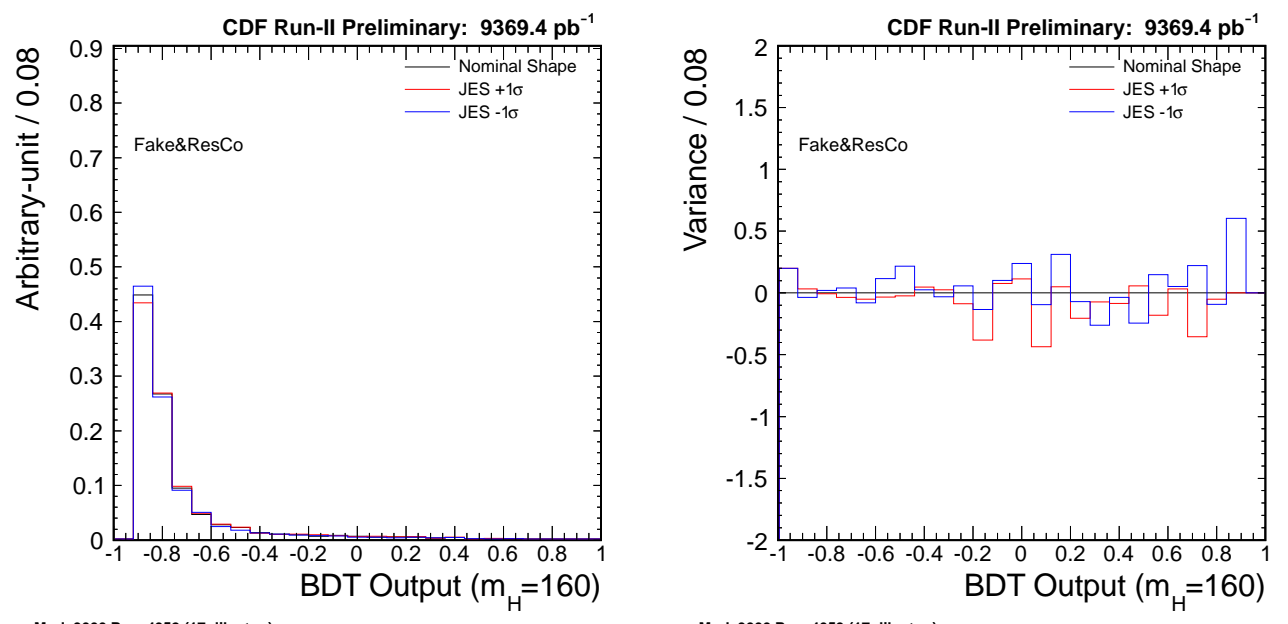

Mode3000.Page4052 (17.dilepton)

Mon May 14 00:30:56 2012 Mode3000.Page4053 (17.dilepton)

Mon May 14 00:34:42 2012
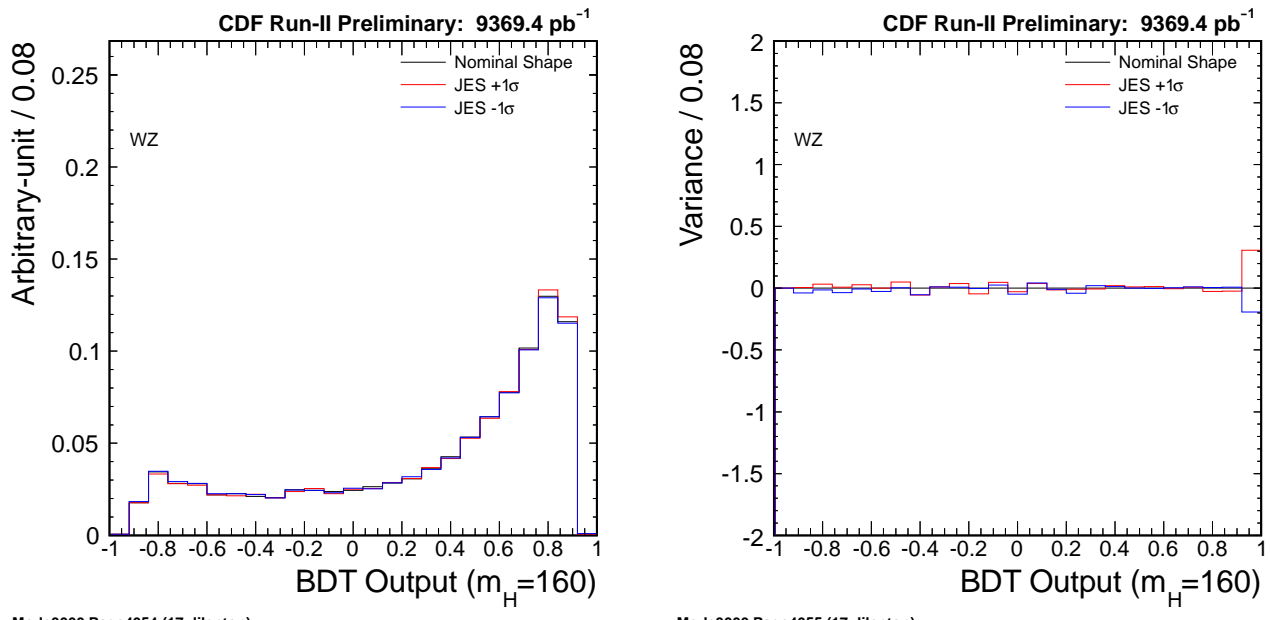

Mode3000.Page4054 (17.dilepton) Mon May 14 00:37:05 2012 Mode3000.Page4055 (17.dilepton)

Mon May 14 00:40:09 2012
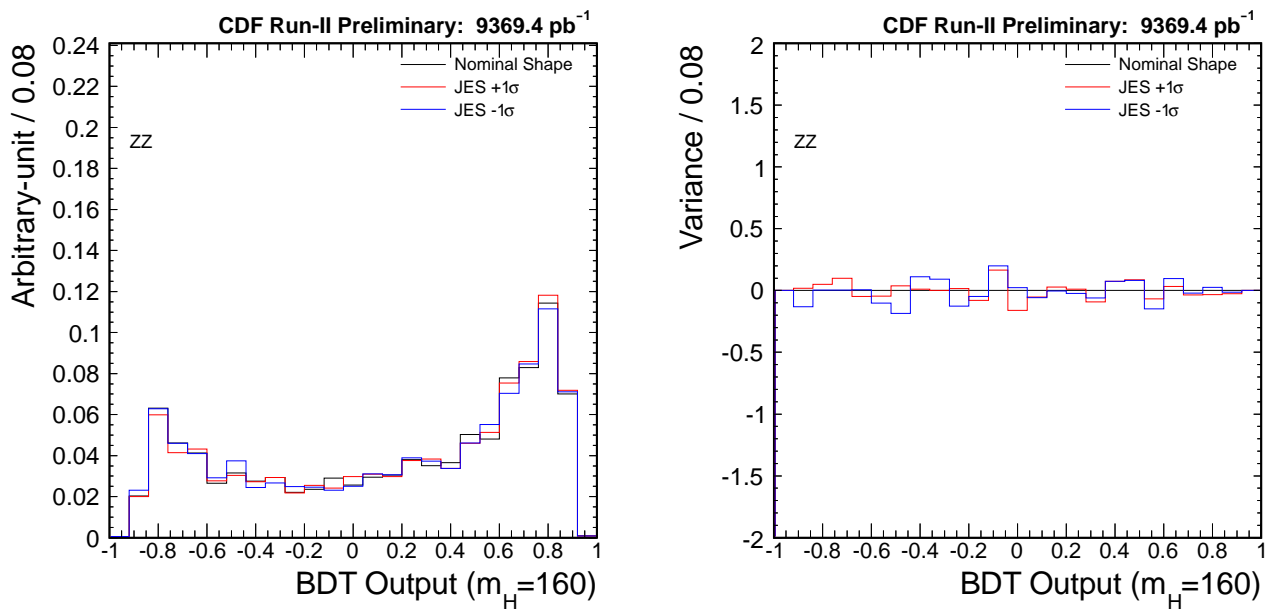

Figure 6.3: Shape systematic uncertainties due to jet energy scale for backgrounds trained on $m_{H}=160 \mathrm{GeV} / c^{2}$. (Fake\&ResCo means fake lepton and residual-photon conversion backgrounds.) 

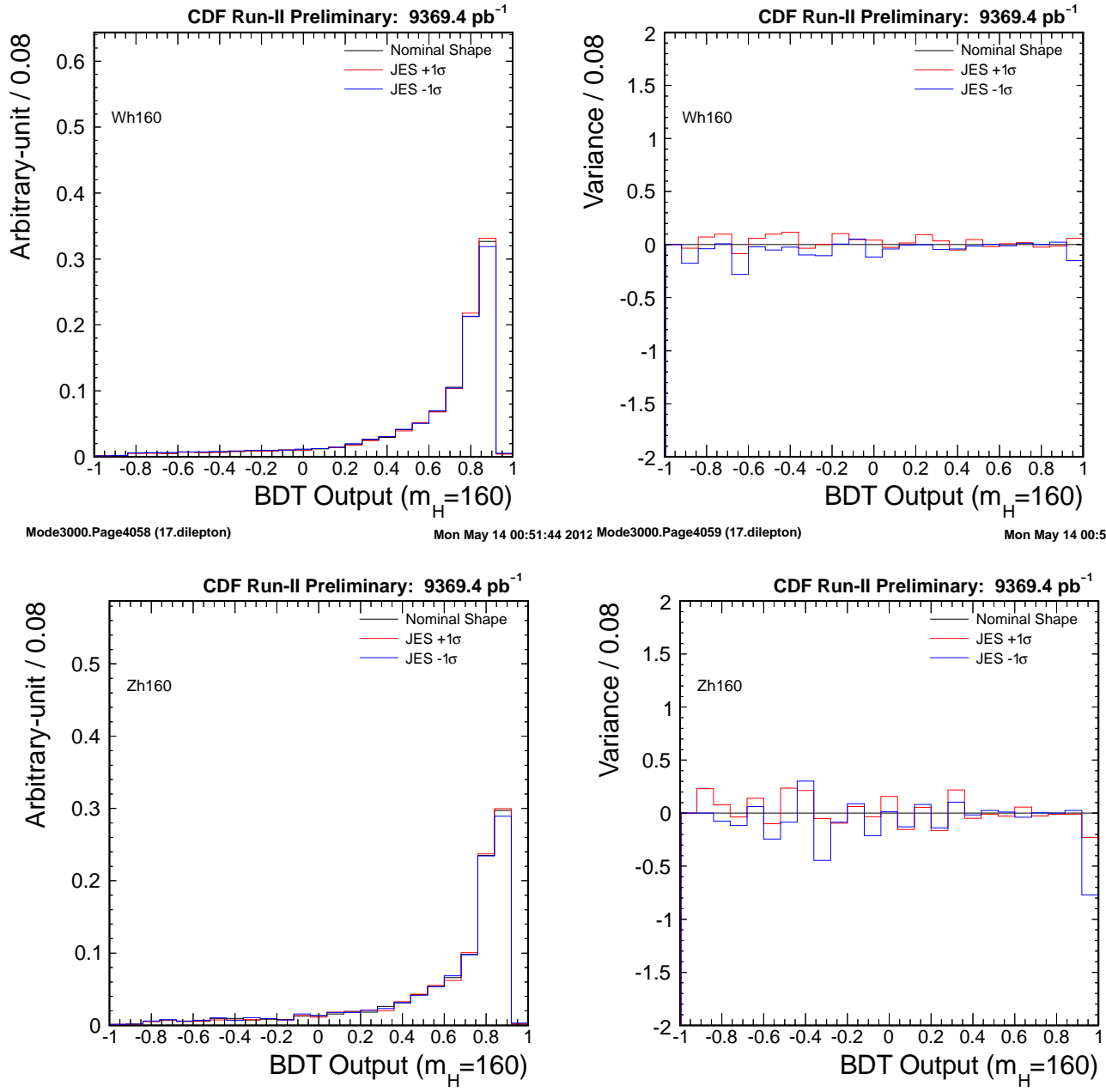

Figure 6.4: Shape systematic uncertainties due to jet energy scale for Higgs Mass 160 $\mathrm{GeV} / c^{2}$. 



\section{Chapter 7}

\section{Results}

As described in the previuos chapters, there is no significance discrepancies between data and background expectation for number of events, several kinematics, and the final discriminant i.e. BDT output. From this result, the upper limits on the Higgs production cross section are set by using binned likelihood function in Bayesian approach using the BDT output distribution. The binned likelihood function obeys the Poisson statistics incorporating some information, that is, systematic uncertainties from several sources by the Gaussian. The following section shows the constriction for the binned likelihood function, and the upper limit on production cross section at a $95 \%$ confidence level by using the likelihood function.

\subsection{Likelihood Function}

The upper limit on production cross section is calculated by using Bayesian approach with fitting binned likelihood to the BDT output [70]. The likelihood is constructed under the Poisson statistics:

$$
p(\mu, n)=\frac{\mu^{n} e^{-\mu}}{n !}, \mu=s+b
$$

where $n$ is number of observed events, $\mu$ is expected number of events, and $s(b)$ is expected number of events for signal(background). In this thesis, the binned likelihood fitting to $N$ bins histogram is written down as the follows,

$$
\mathcal{L}=\prod_{k}^{N_{\text {bin }}} \frac{\mu_{k}^{n_{k}} e^{-\mu_{k}}}{n_{k} !}, \mu_{k}=s_{k}+b_{k}
$$


In addition, the likelihood can be associated with several systematic uncertainties, described in chapter 6 , in terms of Gaussian,

$$
\begin{aligned}
\mathcal{L}\left(\sigma_{1}, \cdots, \sigma_{N_{\text {proc }}} ; \delta_{1}, \cdots, \delta_{N_{\text {syst }}}\right)= & \prod_{k=1}^{N_{\text {bin }}} \frac{\mu_{k}^{n_{k}} \cdot e^{-\mu_{k}}}{n_{k} !} \\
& \cdot \prod_{i=1}^{N_{\text {proc }}} G\left(\sigma_{i} \mid \sigma_{i}^{\mathrm{SM}}, \Delta \sigma_{i}^{\mathrm{SM}}\right) \cdot \prod_{j=1}^{N_{\text {syst }}} G\left(\delta_{j} \mid 0,1\right)
\end{aligned}
$$

where $N_{\text {proc }}$ is number of physics processes, $N_{\text {syst }}$ is number of systematic sources. The expected number of events $\mu_{k}$ is taken both rate and shape systematic uncertainties into account, expressed as

$$
\begin{gathered}
\mu_{k}=\sum_{i=1}^{N_{\text {proc }}} \mu_{i k} \cdot \delta_{i}^{\text {rate }} \cdot \delta_{i k}^{\text {shape }}, \\
\delta_{i}^{\text {rate }}=\prod_{j=1}^{N_{\text {syst }}}\left[1+\left|\delta_{j}\right| \cdot\left\{\varepsilon_{i j+} H\left(\delta_{j}\right)+\varepsilon_{i j-} H\left(-\delta_{j}\right)\right\}\right] \\
\delta_{i k}^{\text {shape }}=\prod_{j=1}^{N_{\text {syst }}}\left[1+\left|\delta_{j}\right| \cdot\left\{\kappa_{i j k+} H\left(\delta_{j}\right)+\kappa_{i j k-} H\left(-\delta_{j}\right)\right\}\right] .
\end{gathered}
$$

where $\varepsilon_{i j}$ is the relative acceptance uncertainties from $j$ th systematic source in $i$ th process, $\kappa_{i j k}$ is the relative uncertainty in the $k$ th bin content from $j$ th systematic source in $i$ th process. Heaviside step function $H\left(\delta_{j}\right)$ is used in the above equations, defined as the follows,

$$
H\left(\delta_{j}\right)= \begin{cases}1 & \left(\delta_{j}>0\right) \\ 0 & \left(\delta_{j}<0\right)\end{cases}
$$

The likelihood function (7.3) is used to calculate the upper limit on the production cross section times branching fraction $\sigma(p \bar{p} \rightarrow W h) \times B_{F}(h \rightarrow W W)$ at a $95 \%$ confidence level.

\subsection{Upper Limit at a 95\% Confidence Level}

In this search, there is no significant excess between the data and the background expectation. Therefore, the upper limits on production cross section times branching fraction $\sigma(p \bar{p} \rightarrow V H) \times B_{F}(H \rightarrow W W)$ at a $95 \%$ confidence level (C.L.) is set by using the binned likelihood function (7.3) in Bayesian approach with the BDT output distribution 
as the following function,

$$
95 \%=\frac{\int_{0}^{\sigma_{95 \%}} \mathcal{L}(\sigma) d \sigma}{\int_{0}^{\infty} \mathcal{L}(\sigma) d \sigma}
$$

The upper limits are calculated for both expected and observed ones corresponding to from 110 to $200 \mathrm{GeV} / c^{2}$ higgs. The expected limit are quoted the median in the distribution for 10,000 pseudo-experiment. As the results, the expected upper limits relative to the theoretical values are

$$
\begin{aligned}
\operatorname{limit}_{(\exp )} / \mathrm{FP}_{\left(m_{H}=110\right)} & <2.6 \\
\operatorname{limit}_{(\mathrm{obs})} / \mathrm{FP}_{\left(m_{H}=110\right)} & <4.4,
\end{aligned}
$$

for the fermiophobic Higgs on the mass of $110 \mathrm{GeV} / c^{2}$, and for the SM Higgs on 160 $\mathrm{GeV} / c^{2}$

$$
\begin{aligned}
& \operatorname{limit}_{(\exp )} / \mathrm{SM}_{\left(m_{H}=160\right)}<5.9 \text {, } \\
& \operatorname{limit}_{(\mathrm{obs})} / \mathrm{SM}_{\left(m_{H}=160\right)}<9.2 \text {. }
\end{aligned}
$$

The relative upper limits to the Higgs prediction cross section times the branching fractions are calculated. For the fermiophobic Higgs, the upper limits relative to the theoretical values are shown in Table 7.1 and Figure 7.1, and for the SM higgs are shown in Table 7.2 and Figure 7.2. 
Table 7.1: The $95 \%$ C.L. limit on the fermiophobic higgs. Including both $W h$ and $Z h$ signals.

\begin{tabular}{ccccccc}
\hline \hline Mass $\left(\mathrm{GeV} / c^{2}\right)$ & \multicolumn{5}{c}{ (Expected limit)/FP } & (Observed limit)/FP \\
& $-2 \sigma$ & $-1 \sigma$ & Median & $+1 \sigma$ & $+2 \sigma$ & \\
\hline 110 & 1.4 & 1.9 & 2.6 & 3.7 & 5.0 & 4.4 \\
120 & 1.6 & 2.2 & 3.0 & 4.3 & 6.0 & 5.3 \\
130 & 1.9 & 2.5 & 3.5 & 4.9 & 7.0 & 5.8 \\
140 & 2.2 & 2.9 & 4.0 & 5.6 & 7.8 & 6.6 \\
150 & 2.6 & 3.4 & 4.7 & 6.7 & 9.3 & 6.6 \\
160 & 3.1 & 4.1 & 5.6 & 8.0 & 11.1 & 8.8 \\
170 & 3.6 & 4.7 & 6.4 & 9.1 & 12.8 & 10.3 \\
180 & 4.4 & 5.7 & 7.8 & 11.1 & 15.5 & 11.3 \\
190 & 6.0 & 7.7 & 10.6 & 15.0 & 20.7 & 16.3 \\
200 & 7.5 & 9.7 & 13.3 & 18.9 & 26.5 & 20.1 \\
\hline \hline
\end{tabular}

Table 7.2: The $95 \%$ C.L. limit on the Standard Model higgs. Including both $W h$ and $Z h$ signals.

\begin{tabular}{ccccccc}
\hline \hline Mass $\left(\mathrm{GeV} / c^{2}\right)$ & \multicolumn{5}{c}{ (Expected limit)/SM } & (Observed limit)/SM \\
& $-2 \sigma$ & $-1 \sigma$ & Median & $+1 \sigma$ & $+2 \sigma$ & \\
\hline 110 & 24.9 & 32.8 & 45.8 & 65.1 & 89.0 & 77.2 \\
120 & 10.0 & 13.2 & 18.3 & 26.1 & 36.2 & 32.5 \\
130 & 5.5 & 7.2 & 10.0 & 14.1 & 19.9 & 16.4 \\
140 & 3.8 & 5.0 & 6.9 & 9.7 & 13.4 & 11.4 \\
150 & 3.3 & 4.3 & 6.0 & 8.5 & 11.8 & 8.3 \\
160 & 3.3 & 4.3 & 5.9 & 8.4 & 11.6 & 9.2 \\
170 & 3.7 & 4.7 & 6.5 & 9.2 & 12.9 & 10.4 \\
180 & 4.5 & 5.7 & 7.9 & 11.2 & 15.6 & 11.4 \\
190 & 6.0 & 7.7 & 10.6 & 15.0 & 20.8 & 16.4 \\
200 & 7.6 & 9.7 & 13.3 & 18.9 & 26.5 & 20.2 \\
\hline \hline
\end{tabular}




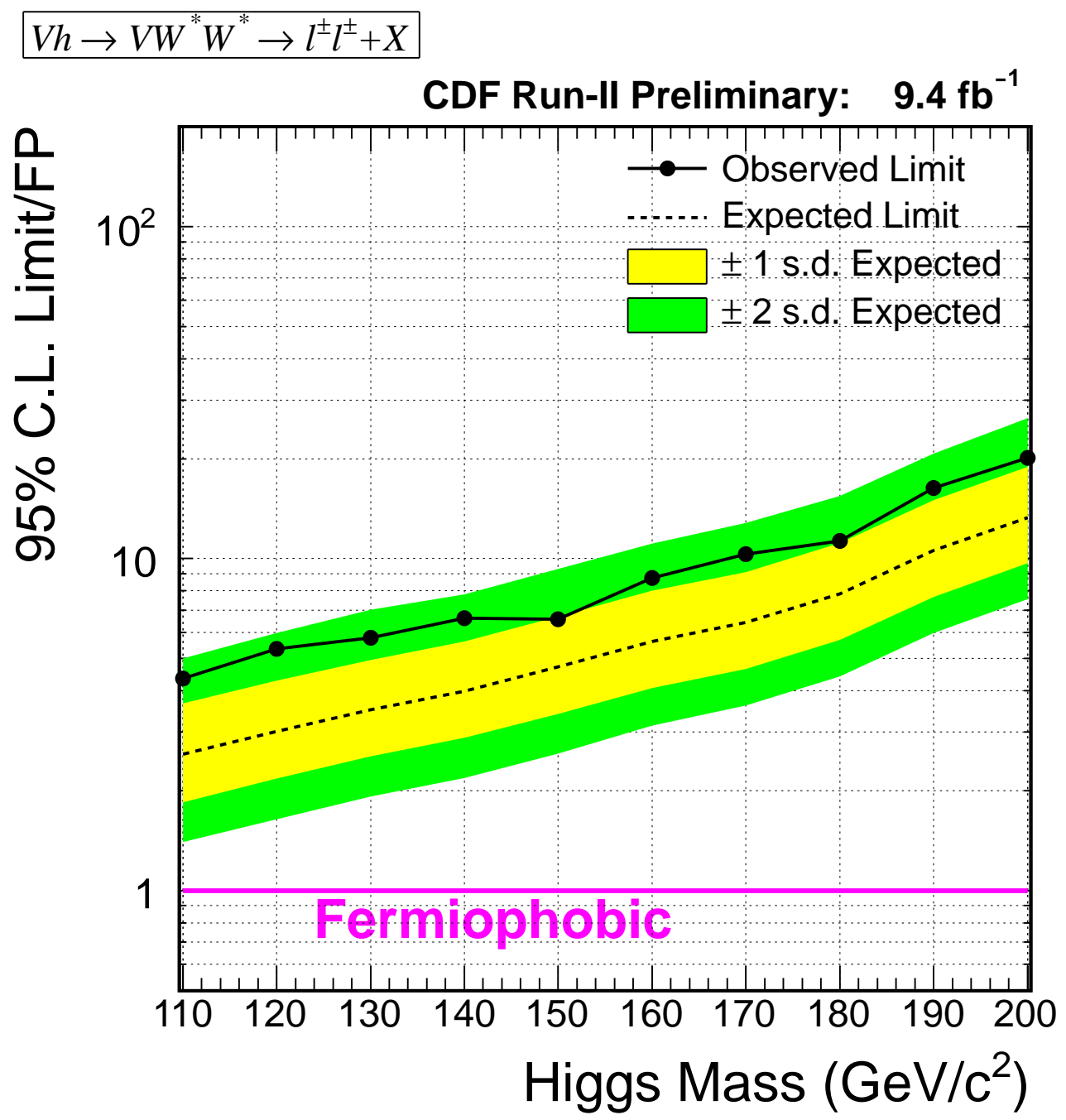

Figure 7.1: Limits on the production cross-section times branching fraction $\sigma(p \bar{p} \rightarrow V h) \times B_{F}\left(h \rightarrow W^{*+} W^{*-}\right)$ to fermiophobic higgs Prediction at the $95 \%$ C.L. 


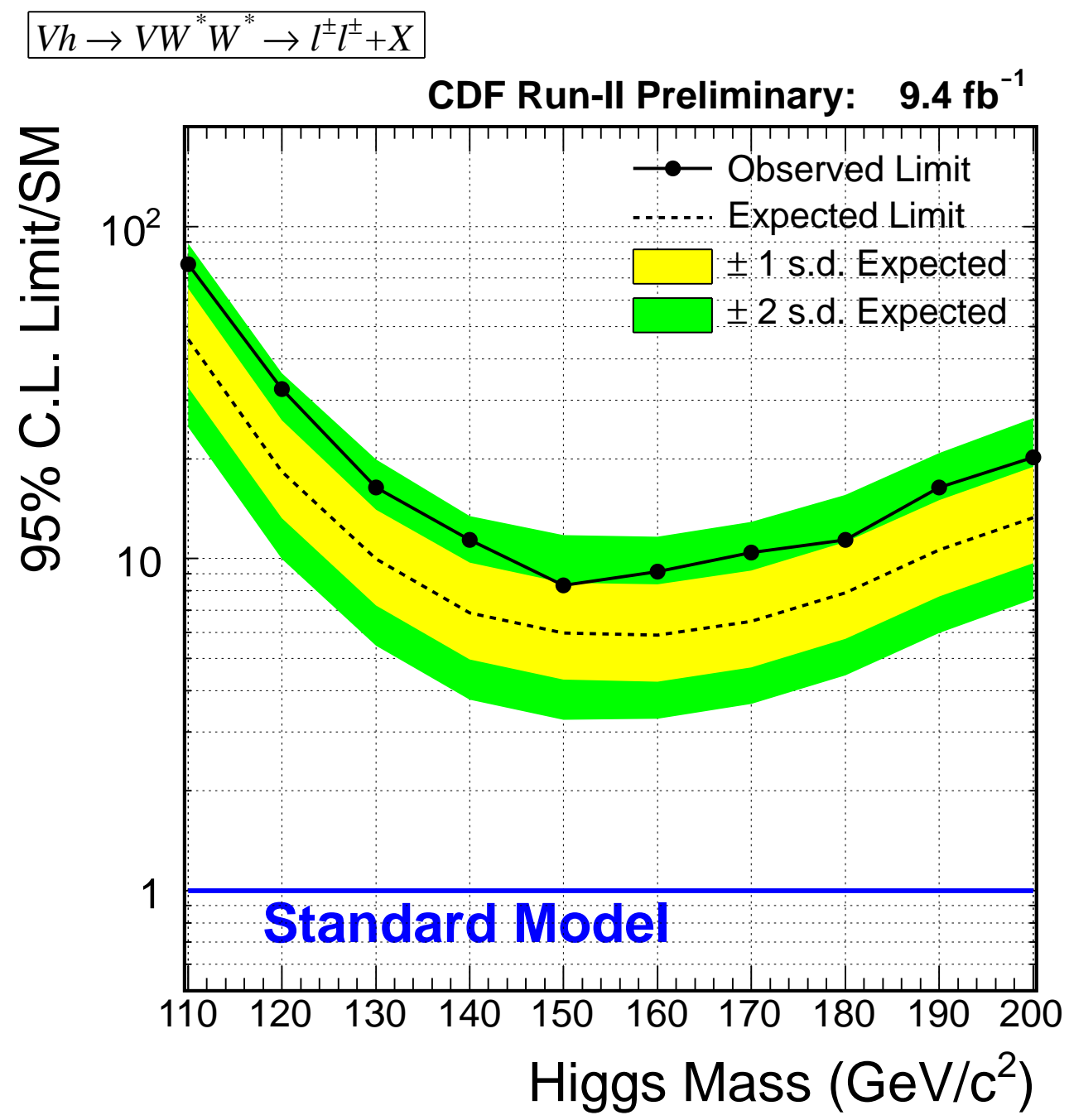

Figure 7.2: Limits on the production cross-section times branching fraction $\sigma(p \bar{p} \rightarrow V h) \times B_{F}\left(h \rightarrow W^{*+} W^{*-}\right)$ to the Standard Model higgs Prediction at the $95 \%$ C.L. 


\section{Chapter 8}

\section{Conclusion}

This thesis has described the search for the neutral Higgs boson production associated with a vector boson using like-sign dilepton events with the data corresponding to an integrated luminosity of $9.4 \mathrm{fb}^{-1}$. The background expectation in the final selected like-sign event was 696.1 events, while the observed was 624 events, so there was no significant discrepancies between data and the expectation within the uncertainties. Also the several kinematical distributions have well agreed. The expected number of signal events was 5.62 for the fermiophobic higgs of the mass $110 \mathrm{GeV} / c^{2}$ assuming the Standard Model production cross section and 1.69 for the Standard Model Higgs boson of $160 \mathrm{GeV} / c^{2}$. The technique of The Boosted Decision Trees was used to give more separation power between backgrounds and signal events in the final sample. The BDT output distribution also have shown no significant excess between the data and background expectation. From this, the upper limits on the production cross section times the branching fraction $\sigma(p \bar{p} \rightarrow W h) \times B_{F}(h \rightarrow W W)$ at a $95 \%$ confidence level was set using the binned likelihood function in Bayesian approach with the BDT output distribution. For the standard model Higgs boson, we set the observed (expected) limit as $9.2(5.9) \times(\mathrm{SM})$ on the mass of $160 \mathrm{GeV} / c^{2}$. Also, we have investigated the fermiophobic Higgs boson scenario, and we set the observed (expected) limit as 4.4 $(2.6) \times(\mathrm{FP})$ on the mass of $110 \mathrm{GeV} / c^{2}$ assuming the standard model cross section of the Higgs boson production. 

Appendix A

Efficiency and Scale Factor for Event Selection

A.1 Trigger Efficiency 
Table A.1: ELECTRON_CENTRAL_18 trigger efficiency for each period.

\begin{tabular}{cc}
\hline \hline Period & Efficiency \\
\hline 0 & $0.960935 \pm 0.001218$ \\
1 & $0.973887 \pm 0.003137$ \\
2 & $0.978824 \pm 0.001711$ \\
3 & $0.972779 \pm 0.002325$ \\
4 & $0.977835 \pm 0.002526$ \\
5 & $0.972909 \pm 0.002820$ \\
6 & $0.976778 \pm 0.002069$ \\
7 & $0.972291 \pm 0.001495$ \\
8 & $0.956808 \pm 0.002099$ \\
9 & $0.956711 \pm 0.002335$ \\
10 & $0.961527 \pm 0.001490$ \\
11 & $0.959706 \pm 0.001926$ \\
12 & $0.958636 \pm 0.002416$ \\
13 & $0.959530 \pm 0.001508$ \\
14 & $0.964156 \pm 0.001495$ \\
15 & $0.965962 \pm 0.001152$ \\
16 & $0.959376 \pm 0.002459$ \\
17 & $0.961422 \pm 0.001917$ \\
18 & $0.960361 \pm 0.001454$ \\
19 & $0.959051 \pm 0.001543$ \\
20 & $0.959731 \pm 0.001622$ \\
21 & $0.956357 \pm 0.001519$ \\
22 & $0.955892 \pm 0.002530$ \\
23 & $0.963495 \pm 0.001096$ \\
24 & $0.961183 \pm 0.001516$ \\
25 & $0.963777 \pm 0.001112$ \\
26 & $0.952256 \pm 0.002000$ \\
27 & $0.952760 \pm 0.001191$ \\
28 & $0.948063 \pm 0.002100$ \\
29 & $0.947860 \pm 0.001957$ \\
30 & $0.947243 \pm 0.000994$ \\
31 & $0.943972 \pm 0.001540$ \\
32 & $0.939068 \pm 0.001326$ \\
33 & $0.937303 \pm 0.001936$ \\
34 & $0.942381 \pm 0.001493$ \\
35 & $0.939620 \pm 0.001295$ \\
36 & $0.938223 \pm 0.001959$ \\
37 & $0.939824 \pm 0.002484$ \\
38 & $0.942169 \pm 0.001481$ \\
\hline \hline &
\end{tabular}


Table A.2: MUON_CMUP18 trigger efficiency for each period.

\begin{tabular}{cc}
\hline \hline Period & Efficiency \\
\hline 0 & $0.894841 \pm 0.013664$ \\
1 & $0.903409 \pm 0.022267$ \\
2 & $0.952381 \pm 0.014012$ \\
3 & $0.935000 \pm 0.017432$ \\
4 & $0.916129 \pm 0.022265$ \\
5 & $0.898678 \pm 0.020028$ \\
6 & $0.935484 \pm 0.019733$ \\
7 & $0.932203 \pm 0.032729$ \\
8 & $0.931596 \pm 0.014407$ \\
9 & $0.914179 \pm 0.017110$ \\
10 & $0.937650 \pm 0.011841$ \\
11 & $0.928375 \pm 0.013534$ \\
12 & $0.910112 \pm 0.017504$ \\
13 & $0.928161 \pm 0.013842$ \\
14 & $0.940476 \pm 0.025815$ \\
15 & $0.930736 \pm 0.016706$ \\
16 & $0.916667 \pm 0.022129$ \\
17 & $0.937984 \pm 0.015015$ \\
18 & $0.924686 \pm 0.012070$ \\
19 & $0.920382 \pm 0.015277$ \\
20 & $0.916399 \pm 0.015695$ \\
21 & $0.913644 \pm 0.011673$ \\
22 & $0.914773 \pm 0.014882$ \\
23 & $0.916667 \pm 0.016636$ \\
24 & $0.920000 \pm 0.015663$ \\
25 & $0.914397 \pm 0.017452$ \\
26 & $0.913876 \pm 0.019406$ \\
27 & $0.922631 \pm 0.011750$ \\
28 & $0.909091 \pm 0.016381$ \\
29 & $0.933661 \pm 0.012336$ \\
30 & $0.892938 \pm 0.014757$ \\
31 & $0.935323 \pm 0.017348$ \\
32 & $0.898230 \pm 0.014221$ \\
33 & $0.912281 \pm 0.015297$ \\
34 & $0.895782 \pm 0.015220$ \\
35 & $0.878261 \pm 0.017604$ \\
36 & $0.902941 \pm 0.016055$ \\
37 & $0.929730 \pm 0.018792$ \\
38 & $0.882845 \pm 0.020803$ \\
\hline \hline &
\end{tabular}


Table A.3: MUON_CMX18 trigger efficiency for each period.

\begin{tabular}{cc}
\hline \hline Period & Efficiency \\
\hline 0 & $0.985386 \pm 0.005483$ \\
1 & $0.914894 \pm 0.020351$ \\
2 & $0.890909 \pm 0.021018$ \\
3 & $0.862245 \pm 0.024617$ \\
4 & $0.938776 \pm 0.019774$ \\
5 & $0.894737 \pm 0.020324$ \\
6 & $0.878453 \pm 0.024288$ \\
7 & $0.823529 \pm 0.053381$ \\
8 & $0.882353 \pm 0.017927$ \\
9 & $0.865455 \pm 0.020577$ \\
10 & $0.936170 \pm 0.012607$ \\
11 & $0.912568 \pm 0.014765$ \\
12 & $0.890351 \pm 0.020693$ \\
13 & $0.839793 \pm 0.018645$ \\
14 & $0.906667 \pm 0.033590$ \\
15 & $0.858974 \pm 0.022753$ \\
16 & $0.925170 \pm 0.021702$ \\
17 & $0.905109 \pm 0.017705$ \\
18 & $0.907258 \pm 0.013025$ \\
19 & $0.871875 \pm 0.018684$ \\
20 & $0.894040 \pm 0.017711$ \\
21 & $0.891200 \pm 0.012456$ \\
22 & $0.915452 \pm 0.015022$ \\
23 & $0.930041 \pm 0.016363$ \\
24 & $0.902985 \pm 0.018080$ \\
25 & $0.906615 \pm 0.018150$ \\
26 & $0.879070 \pm 0.022236$ \\
27 & $0.889465 \pm 0.013031$ \\
28 & $0.908163 \pm 0.016843$ \\
29 & $0.835509 \pm 0.018943$ \\
30 & $0.821721 \pm 0.017326$ \\
31 & $0.830000 \pm 0.026561$ \\
32 & $0.849593 \pm 0.016116$ \\
33 & $0.826733 \pm 0.018830$ \\
34 & $0.878713 \pm 0.016242$ \\
35 & $0.857143 \pm 0.018894$ \\
36 & $0.829016 \pm 0.019163$ \\
37 & $0.810526 \pm 0.028430$ \\
38 & $0.837945 \pm 0.023167$ \\
\hline \hline &
\end{tabular}




\section{A.2 Primary-Vertex Cut Efficiency and Scale Fac- tor}

Table A.4: Primary vertex cut efficiency and scale factor for 0d data set.

\begin{tabular}{cc}
\hline \hline High- $p_{T}$ Electron Efficiency & $0.995011 \pm 0.000090$ \\
High- $p_{T}$ Muon Efficiency & $0.98624 \pm 0.00028$ \\
\hline Data Average Efficiency & $0.9906 \pm 0.0062$ \\
\hline Drell-Yan $\left(Z / \gamma^{*} \rightarrow e^{+} e^{-}\right)$MC Efficiency & $0.97756 \pm 0.00020$ \\
Drell-Yan $\left(Z / \gamma^{*} \rightarrow \mu^{+} \mu^{-}\right)$MC Efficiency & $0.96082 \pm 0.00031$ \\
\hline Drell-Yan MC Average Efficiency & $0.969 \pm 0.012$ \\
\hline Scale factor & $1.022 \pm 0.014$ \\
\hline \hline
\end{tabular}

Table A.5: Primary vertex cut efficiency and scale factor for Oh data set.

\begin{tabular}{cc}
\hline \hline High- $p_{T}$ Electron Efficiency & $0.995975 \pm 0.000083$ \\
High- $p_{T}$ Muon Efficiency & $0.98848 \pm 0.00022$ \\
\hline Data Average Efficiency & $0.9922 \pm 0.0053$ \\
\hline Drell-Yan $\left(Z / \gamma^{*} \rightarrow e^{+} e^{-}\right)$MC Efficiency & $0.98115 \pm 0.00016$ \\
Drell-Yan $\left(Z / \gamma^{*} \rightarrow \mu^{+} \mu^{-}\right)$MC Efficiency & $0.96773 \pm 0.00024$ \\
\hline Drell-Yan MC Average Efficiency & $0.9744 \pm 0.0095$ \\
\hline Scale factor & $1.018 \pm 0.011$ \\
\hline \hline
\end{tabular}

Table A.6: Primary vertex cut efficiency and scale factor for 0i data set.

\begin{tabular}{cc}
\hline \hline High- $p_{T}$ Electron Efficiency & $0.996337 \pm 0.000066$ \\
High- $p_{T}$ Muon Efficiency & $0.98858 \pm 0.00018$ \\
\hline Data Average Efficiency & $0.9925 \pm 0.0055$ \\
\hline Drell-Yan $\left(Z / \gamma^{*} \rightarrow e^{+} e^{-}\right)$MC Efficiency & $0.98251 \pm 0.00013$ \\
Drell-Yan $\left(Z / \gamma^{*} \rightarrow \mu^{+} \mu^{-}\right)$MC Efficiency & $0.97037 \pm 0.00019$ \\
\hline Drell-Yan MC Average Efficiency & $0.9764 \pm 0.0086$ \\
\hline Scale factor & $1.016 \pm 0.011$ \\
\hline \hline
\end{tabular}


Table A.7: Primary vertex cut efficiency and scale factor for $0 j$ data set.

\begin{tabular}{cc}
\hline \hline High- $p_{T}$ Electron Efficiency & $0.996333 \pm 0.000055$ \\
High- $p_{T}$ Muon Efficiency & $0.98806 \pm 0.00016$ \\
\hline Data Average Efficiency & $0.9922 \pm 0.0058$ \\
\hline Drell-Yan $\left(Z / \gamma^{*} \rightarrow e^{+} e^{-}\right)$MC Efficiency & $0.985229 \pm 0.000096$ \\
Drell-Yan $\left(Z / \gamma^{*} \rightarrow \mu^{+} \mu^{-}\right)$MC Efficiency & $0.97533 \pm 0.00014$ \\
\hline Drell-Yan MC Average Efficiency & $0.9803 \pm 0.0070$ \\
\hline Scale factor & $1.0122 \pm 0.0094$ \\
\hline \hline
\end{tabular}

Table A.8: Primary vertex cut efficiency and scale factor for 0k data set.

\begin{tabular}{cc}
\hline \hline High- $p_{T}$ Electron Efficiency & $0.997300 \pm 0.000063$ \\
High- $p_{T}$ Muon Efficiency & $0.98980 \pm 0.00020$ \\
\hline Data Average Efficiency & $0.9936 \pm 0.0053$ \\
\hline Drell-Yan $\left(Z / \gamma^{*} \rightarrow e^{+} e^{-}\right)$MC Efficiency & $0.98543 \pm 0.00011$ \\
Drell-Yan $\left(Z / \gamma^{*} \rightarrow \mu^{+} \mu^{-}\right)$MC Efficiency & $0.97550 \pm 0.00016$ \\
\hline Drell-Yan MC Average Efficiency & $0.9805 \pm 0.0070$ \\
\hline Scale factor & $1.0133 \pm 0.0090$ \\
\hline \hline
\end{tabular}

Table A.9: Primary vertex cut efficiency and scale factor for 0m data set.

\begin{tabular}{cc}
\hline \hline $\begin{array}{c}\text { High- } p_{T} \text { Electron Efficiency } \\
\text { High- } p_{T} \text { Muon Efficiency }\end{array}$ & $0.997221 \pm 0.000027$ \\
Data Average Efficiency & $0.990140 \pm 0.000082$ \\
\hline Drell-Yan $\left(Z / \gamma^{*} \rightarrow e^{+} e^{-}\right)$MC Efficiency & $0.98543 \pm 0.0050$ \\
Drell-Yan $\left(Z / \gamma^{*} \rightarrow \mu^{+} \mu^{-}\right)$MC Efficiency & $0.97550 \pm 0.00011$ \\
\hline Drell-Yan MC Average Efficiency & $0.9805 \pm 0.0070$ \\
\hline Scale factor & $1.0135 \pm 0.0089$ \\
\hline \hline
\end{tabular}


Table A.10: Primary vertex cut efficiency and scale factor for $0 \mathrm{p}$ data set.

\begin{tabular}{cc}
\hline \hline High- $p_{T}$ Electron Efficiency & $0.996930 \pm 0.000028$ \\
High- $p_{T}$ Muon Efficiency & $0.989201 \pm 0.000089$ \\
\hline Data Average Efficiency & $0.9931 \pm 0.0055$ \\
\hline Drell-Yan $\left(Z / \gamma^{*} \rightarrow e^{+} e^{-}\right)$MC Efficiency & $0.98543 \pm 0.00011$ \\
Drell-Yan $\left(Z / \gamma^{*} \rightarrow \mu^{+} \mu^{-}\right)$MC Efficiency & $0.97550 \pm 0.00016$ \\
\hline Drell-Yan MC Average Efficiency & $0.9805 \pm 0.0070$ \\
\hline Scale factor & $1.0129 \pm 0.0091$ \\
\hline \hline
\end{tabular}




\section{A.3 Lepton Selection Efficiency and Scale Factor}

\section{A.3.1 Electron Selection Efficiency and Scale Factor}

Table A.11: Low $E_{T}$ electron selection cut efficiencies and scale factors for 0d data set.

\begin{tabular}{cccc}
\hline \hline Cut & Data Efficiency & MC Efficiency & Scale factor \\
\hline COT hit & $0.960 \pm 0.011$ & $0.99887 \pm 0.00025$ & $0.961 \pm 0.011$ \\
$z_{0}-z_{\mathrm{pv}}$ & $0.9741 \pm 0.0070$ & $0.99101 \pm 0.00069$ & $0.9830 \pm 0.0071$ \\
$d_{0}$ & $0.941 \pm 0.013$ & $0.9442 \pm 0.0017$ & $0.996 \pm 0.014$ \\
Silicon hit & $0.871 \pm 0.018$ & $0.9082 \pm 0.0021$ & $0.959 \pm 0.020$ \\
ISO $_{0.4}^{\text {cal }}$ & $0.675 \pm 0.025$ & $0.7115 \pm 0.0034$ & $0.949 \pm 0.036$ \\
Track Quality \& ISO cal & $0.606 \pm 0.024$ & $0.6314 \pm 0.0036$ & $0.960 \pm 0.039$ \\
\hline Conversion removal (CR) & $0.925 \pm 0.011$ & $0.9840 \pm 0.0012$ & $0.940 \pm 0.011$ \\
Likelihood ID (LID) & $0.874 \pm 0.014$ & $0.8960 \pm 0.0028$ & $0.976 \pm 0.016$ \\
Overall & $0.606 \pm 0.024$ & $0.6314 \pm 0.0036$ & $0.960 \pm 0.039$ \\
\hline \hline
\end{tabular}

Table A.12: High $E_{T}$ electron selection cut efficiencies and scale factors for Od data set.

\begin{tabular}{cccc}
\hline \hline Cut & Data Efficiency & MC Efficiency & Scale factor \\
\hline COT hit & $0.9747 \pm 0.0022$ & $0.999226 \pm 0.000084$ & $0.9754 \pm 0.0022$ \\
$z_{0}-z_{\mathrm{pv}}$ & $0.9938 \pm 0.0013$ & $0.99277 \pm 0.00026$ & $1.0010 \pm 0.0013$ \\
$d_{0}$ & $0.9598 \pm 0.0030$ & $0.95659 \pm 0.00062$ & $1.0034 \pm 0.0032$ \\
Silicon hit & $0.8949 \pm 0.0042$ & $0.91249 \pm 0.00086$ & $0.9808 \pm 0.0047$ \\
ISO $_{0.4}^{\text {cal }}$ & $0.8571 \pm 0.0049$ & $0.8573 \pm 0.0011$ & $0.9997 \pm 0.0059$ \\
Track Quality \& ISO cal & $0.7675 \pm 0.0058$ & $0.7621 \pm 0.0013$ & $1.0070 \pm 0.0078$ \\
\hline Conversion removal (CR) & $0.7936 \pm 0.0059$ & $0.92324 \pm 0.00093$ & $0.8596 \pm 0.0065$ \\
Likelihood ID (LID) & $0.9754 \pm 0.0023$ & $0.98298 \pm 0.00045$ & $0.9923 \pm 0.0024$ \\
Overall & $0.7675 \pm 0.0058$ & $0.7621 \pm 0.0013$ & $1.0070 \pm 0.0078$ \\
\hline \hline
\end{tabular}


Table A.13: Low $E_{T}$ electron selection cut efficiencies and scale factors for $0 \mathrm{~h}$ data set.

\begin{tabular}{cccc}
\hline \hline Cut & Data Efficiency & MC Efficiency & Scale factor \\
\hline COT hit & $1.00 \pm 0.00$ & $0.99876 \pm 0.00023$ & $1.00124 \pm 0.00023$ \\
$z_{0}-z_{\mathrm{pv}}$ & $0.9940 \pm 0.0055$ & $0.99163 \pm 0.00060$ & $1.0024 \pm 0.0056$ \\
$d_{0}$ & $0.956 \pm 0.014$ & $0.9455 \pm 0.0015$ & $1.011 \pm 0.014$ \\
Silicon hit & $0.927 \pm 0.014$ & $0.9182 \pm 0.0018$ & $1.010 \pm 0.016$ \\
ISO $_{0.4}^{\text {al }}$ & $0.683 \pm 0.024$ & $0.7060 \pm 0.0030$ & $0.968 \pm 0.034$ \\
Track Quality \& ISO 0.4 & $0.619 \pm 0.023$ & $0.6326 \pm 0.0032$ & $0.978 \pm 0.037$ \\
\hline Conversion removal $(\mathrm{CR})$ & $0.923 \pm 0.010$ & $0.98718 \pm 0.00095$ & $0.935 \pm 0.010$ \\
Likelihood ID (LID) & $0.880 \pm 0.013$ & $0.8926 \pm 0.0026$ & $0.986 \pm 0.015$ \\
Overall & $0.619 \pm 0.023$ & $0.6326 \pm 0.0032$ & $0.978 \pm 0.037$ \\
\hline \hline
\end{tabular}

Table A.14: High $E_{T}$ electron selection cut efficiencies and scale factors for $0 \mathrm{~h}$ data set.

\begin{tabular}{cccc}
\hline \hline Cut & Data Efficiency & MC Efficiency & Scale factor \\
\hline COT hit & $0.99732 \pm 0.00069$ & $0.999028 \pm 0.000085$ & $0.99829 \pm 0.00069$ \\
$z_{0}-z_{\mathrm{pv}}$ & $0.9904 \pm 0.0013$ & $0.99288 \pm 0.00023$ & $0.9975 \pm 0.0014$ \\
$d_{0}$ & $0.9640 \pm 0.0028$ & $0.95804 \pm 0.00055$ & $1.0062 \pm 0.0030$ \\
Silicon hit & $0.9303 \pm 0.0034$ & $0.92147 \pm 0.00074$ & $1.0096 \pm 0.0038$ \\
ISO $_{0.4}^{\text {cal }}$ & $0.8615 \pm 0.0046$ & $0.84667 \pm 0.00099$ & $1.0176 \pm 0.0056$ \\
Track Quality \& ISO cal & $0.7850 \pm 0.0054$ & $0.7619 \pm 0.0012$ & $1.0303 \pm 0.0073$ \\
\hline Conversion removal (CR) & $0.8038 \pm 0.0055$ & $0.94782 \pm 0.00070$ & $0.8480 \pm 0.0058$ \\
Likelihood ID (LID) & $0.9737 \pm 0.0022$ & $0.97839 \pm 0.00046$ & $0.9952 \pm 0.0023$ \\
Overall & $0.7850 \pm 0.0054$ & $0.7619 \pm 0.0012$ & $1.0303 \pm 0.0073$ \\
\hline \hline
\end{tabular}


Table A.15: Low $E_{T}$ electron selection cut efficiencies and scale factors for 0 i data set.

\begin{tabular}{cccc}
\hline \hline Cut & Data Efficiency & MC Efficiency & Scale factor \\
\hline COT hit & $0.9996 \pm 0.0046$ & $0.99824 \pm 0.00023$ & $1.0014 \pm 0.0046$ \\
$z_{0}-z_{\mathrm{pv}}$ & $0.9888 \pm 0.0047$ & $0.99108 \pm 0.00051$ & $0.9977 \pm 0.0048$ \\
$d_{0}$ & $0.953 \pm 0.010$ & $0.9443 \pm 0.0013$ & $1.009 \pm 0.011$ \\
Silicon hit & $0.936 \pm 0.013$ & $0.9165 \pm 0.0015$ & $1.021 \pm 0.014$ \\
ISO $_{0.4}^{\text {cal }}$ & $0.674 \pm 0.020$ & $0.7032 \pm 0.0025$ & $0.958 \pm 0.029$ \\
Track Quality \& ISO 0.4 & $0.609 \pm 0.020$ & $0.6289 \pm 0.0026$ & $0.968 \pm 0.031$ \\
\hline Conversion removal (CR) & $0.8922 \pm 0.0098$ & $0.9553 \pm 0.0014$ & $0.934 \pm 0.010$ \\
Likelihood ID (LID) & $0.872 \pm 0.011$ & $0.8930 \pm 0.0021$ & $0.977 \pm 0.013$ \\
Overall & $0.609 \pm 0.020$ & $0.6289 \pm 0.0026$ & $0.968 \pm 0.031$ \\
\hline \hline
\end{tabular}

Table A.16: High $E_{T}$ electron selection cut efficiencies and scale factors for $0 \mathrm{~h}$ data set.

\begin{tabular}{cccc}
\hline \hline Cut & Data Efficiency & MC Efficiency & Scale factor \\
\hline COT hit & $0.99623 \pm 0.00066$ & $0.998872 \pm 0.000075$ & $0.99735 \pm 0.00066$ \\
$z_{0}-z_{\mathrm{pv}}$ & $0.9909 \pm 0.0012$ & $0.99217 \pm 0.00020$ & $0.9987 \pm 0.0012$ \\
$d_{0}$ & $0.9625 \pm 0.0023$ & $0.95697 \pm 0.00045$ & $1.0058 \pm 0.0025$ \\
Silicon hit & $0.9261 \pm 0.0029$ & $0.91896 \pm 0.00061$ & $1.0078 \pm 0.0032$ \\
ISO $_{0.4}^{\text {cal }}$ & $0.8406 \pm 0.0039$ & $0.83393 \pm 0.00083$ & $1.0080 \pm 0.0048$ \\
Track Quality \& ISO cal & $0.7637 \pm 0.0045$ & $0.74773 \pm 0.00097$ & $1.0213 \pm 0.0062$ \\
\hline Conversion removal (CR) & $0.7908 \pm 0.0046$ & $0.8079 \pm 0.0010$ & $0.9788 \pm 0.0059$ \\
Likelihood ID (LID) & $0.9712 \pm 0.0019$ & $0.97923 \pm 0.00037$ & $0.9918 \pm 0.0020$ \\
Overall & $0.7637 \pm 0.0045$ & $0.74773 \pm 0.00097$ & $1.0213 \pm 0.0062$ \\
\hline \hline
\end{tabular}


Table A.17: Low $E_{T}$ electron selection cut efficiencies and scale factors for $0 \mathrm{j}$ data set.

\begin{tabular}{cccc}
\hline \hline Cut & Data Efficiency & MC Efficiency & Scale factor \\
\hline COT hit & $0.9970 \pm 0.0031$ & $0.99805 \pm 0.00020$ & $0.9990 \pm 0.0031$ \\
$z_{0}-z_{\mathrm{pv}}$ & $0.9935 \pm 0.0048$ & $0.99100 \pm 0.00043$ & $1.0025 \pm 0.0048$ \\
$d_{0}$ & $0.9640 \pm 0.0094$ & $0.9461 \pm 0.0010$ & $1.019 \pm 0.010$ \\
Silicon hit & $0.921 \pm 0.011$ & $0.9083 \pm 0.0013$ & $1.014 \pm 0.012$ \\
ISO $_{0.4}^{\text {cal }}$ & $0.668 \pm 0.017$ & $0.6795 \pm 0.0021$ & $0.983 \pm 0.026$ \\
Track Quality \& ISO cal & $0.598 \pm 0.017$ & $0.6060 \pm 0.0022$ & $0.988 \pm 0.027$ \\
\hline Conversion removal (CR) & $0.9322 \pm 0.0070$ & $0.9624 \pm 0.0011$ & $0.9687 \pm 0.0074$ \\
Likelihood ID (LID) & $0.8939 \pm 0.0096$ & $0.8932 \pm 0.0018$ & $1.001 \pm 0.011$ \\
Overall & $0.598 \pm 0.017$ & $0.6060 \pm 0.0022$ & $0.988 \pm 0.027$ \\
\hline \hline
\end{tabular}

Table A.18: High $E_{T}$ electron selection cut efficiencies and scale factors for $0 \mathrm{j}$ data set.

\begin{tabular}{cccc}
\hline \hline Cut & Data Efficiency & MC Efficiency & Scale factor \\
\hline COT hit & $0.99695 \pm 0.00063$ & $0.998407 \pm 0.000074$ & $0.99854 \pm 0.00064$ \\
$z_{0}-z_{\mathrm{pv}}$ & $0.9896 \pm 0.0010$ & $0.99240 \pm 0.00016$ & $0.9972 \pm 0.0010$ \\
$d_{0}$ & $0.9672 \pm 0.0020$ & $0.95927 \pm 0.00036$ & $1.0083 \pm 0.0021$ \\
Silicon hit & $0.9245 \pm 0.0025$ & $0.91160 \pm 0.00052$ & $1.0142 \pm 0.0028$ \\
ISO $_{0.4}^{\text {cal }}$ & $0.8096 \pm 0.0036$ & $0.81291 \pm 0.00072$ & $0.9959 \pm 0.0045$ \\
Track Quality \& ISO cal & $0.7319 \pm 0.0040$ & $0.72530 \pm 0.00082$ & $1.0091 \pm 0.0056$ \\
\hline Conversion removal (CR) & $0.8387 \pm 0.0037$ & $0.85396 \pm 0.00076$ & $0.9821 \pm 0.0044$ \\
Likelihood ID (LID) & $0.9692 \pm 0.0017$ & $0.97851 \pm 0.00031$ & $0.9905 \pm 0.0018$ \\
Overall & $0.7319 \pm 0.0040$ & $0.72530 \pm 0.00082$ & $1.0091 \pm 0.0056$ \\
\hline \hline
\end{tabular}


Table A.19: Low $E_{T}$ electron selection cut efficiencies and scale factors for 0k data set.

\begin{tabular}{cccc}
\hline \hline Cut & Data Efficiency & MC Efficiency & Scale factor \\
\hline COT hit & $1.00 \pm 0.00$ & $0.99736 \pm 0.00026$ & $1.00264 \pm 0.00026$ \\
$z_{0}-z_{\mathrm{pv}}$ & $0.9968 \pm 0.0067$ & $0.99073 \pm 0.00048$ & $1.0061 \pm 0.0068$ \\
$d_{0}$ & $0.946 \pm 0.013$ & $0.9461 \pm 0.0011$ & $1.000 \pm 0.014$ \\
Silicon hit & $0.938 \pm 0.014$ & $0.9136 \pm 0.0014$ & $1.027 \pm 0.016$ \\
ISO $_{0.4}^{\text {cal }}$ & $0.631 \pm 0.022$ & $0.6600 \pm 0.0024$ & $0.955 \pm 0.033$ \\
Track Quality \& ISO 0.4 & $0.577 \pm 0.021$ & $0.5912 \pm 0.0025$ & $0.976 \pm 0.036$ \\
\hline Conversion removal (CR) & $0.921 \pm 0.010$ & $0.97935 \pm 0.00094$ & $0.940 \pm 0.011$ \\
Likelihood ID (LID) & $0.886 \pm 0.013$ & $0.8904 \pm 0.0020$ & $0.995 \pm 0.015$ \\
Overall & $0.577 \pm 0.021$ & $0.5912 \pm 0.0025$ & $0.976 \pm 0.036$ \\
\hline \hline
\end{tabular}

Table A.20: High $E_{T}$ electron selection cut efficiencies and scale factors for 0k data set.

\begin{tabular}{cccc}
\hline \hline Cut & Data Efficiency & MC Efficiency & Scale factor \\
\hline COT hit & $0.99639 \pm 0.00086$ & $0.997959 \pm 0.000093$ & $0.99842 \pm 0.00087$ \\
$z_{0}-z_{\mathrm{pv}}$ & $0.9894 \pm 0.0013$ & $0.99223 \pm 0.00018$ & $0.9972 \pm 0.0014$ \\
$d_{0}$ & $0.9602 \pm 0.0026$ & $0.95832 \pm 0.00041$ & $1.0019 \pm 0.0027$ \\
Silicon hit & $0.9245 \pm 0.0032$ & $0.91822 \pm 0.00056$ & $1.0068 \pm 0.0035$ \\
ISO $_{0.4}^{\text {cal }}$ & $0.8099 \pm 0.0047$ & $0.79147 \pm 0.00083$ & $1.0233 \pm 0.0060$ \\
Track Quality \& ISO cal & $0.7343 \pm 0.0052$ & $0.70975 \pm 0.00093$ & $1.0346 \pm 0.0075$ \\
\hline Conversion removal (CR) & $0.8286 \pm 0.0050$ & $0.91895 \pm 0.00066$ & $0.9017 \pm 0.0055$ \\
Likelihood ID (LID) & $0.9656 \pm 0.0024$ & $0.97813 \pm 0.00036$ & $0.9872 \pm 0.0025$ \\
Overall & $0.7343 \pm 0.0052$ & $0.70975 \pm 0.00093$ & $1.0346 \pm 0.0075$ \\
\hline \hline
\end{tabular}


Table A.21: Low $E_{T}$ electron selection cut efficiencies and scale factors for 0m data set.

\begin{tabular}{cccc}
\hline \hline Cut & Data Efficiency & MC Efficiency & Scale factor \\
\hline COT hit & $0.9946 \pm 0.0020$ & $0.99736 \pm 0.00026$ & $0.9972 \pm 0.0021$ \\
$z_{0}-z_{\mathrm{pv}}$ & $0.9877 \pm 0.0029$ & $0.99073 \pm 0.00048$ & $0.9969 \pm 0.0030$ \\
$d_{0}$ & $0.9595 \pm 0.0055$ & $0.9461 \pm 0.0011$ & $1.0141 \pm 0.0059$ \\
Silicon hit & $0.9120 \pm 0.0064$ & $0.9136 \pm 0.0014$ & $0.9982 \pm 0.0072$ \\
ISO $_{0.4}^{\text {cal }}$ & $0.5999 \pm 0.0092$ & $0.6600 \pm 0.0024$ & $0.909 \pm 0.014$ \\
Track Quality \& ISO cal & $0.5326 \pm 0.0087$ & $0.5912 \pm 0.0025$ & $0.901 \pm 0.015$ \\
\hline Conversion removal (CR) & $0.9445 \pm 0.0041$ & $0.97935 \pm 0.00094$ & $0.9644 \pm 0.0043$ \\
Likelihood ID (LID) & $0.8830 \pm 0.0059$ & $0.8904 \pm 0.0020$ & $0.9917 \pm 0.0070$ \\
Overall & $0.5326 \pm 0.0087$ & $0.5912 \pm 0.0025$ & $0.901 \pm 0.015$ \\
\hline \hline
\end{tabular}

Table A.22: High $E_{T}$ electron selection cut efficiencies and scale factors for $0 \mathrm{~m}$ data set.

\begin{tabular}{cccc}
\hline \hline Cut & Data Efficiency & MC Efficiency & Scale factor \\
\hline COT hit & $0.99494 \pm 0.00041$ & $0.997959 \pm 0.000093$ & $0.99697 \pm 0.00042$ \\
$z_{0}-z_{\mathrm{pv}}$ & $0.98785 \pm 0.00065$ & $0.99223 \pm 0.00018$ & $0.99559 \pm 0.00068$ \\
$d_{0}$ & $0.9603 \pm 0.0012$ & $0.95832 \pm 0.00041$ & $1.0020 \pm 0.0013$ \\
Silicon hit & $0.9024 \pm 0.0016$ & $0.91822 \pm 0.00056$ & $0.9828 \pm 0.0018$ \\
ISO $_{0.4}^{\text {cal }}$ & $0.7647 \pm 0.0022$ & $0.79147 \pm 0.00083$ & $0.9662 \pm 0.0030$ \\
Track Quality \& ISO cal & $0.6766 \pm 0.0024$ & $0.70975 \pm 0.00093$ & $0.9533 \pm 0.0036$ \\
\hline Conversion removal (CR) & $0.9410 \pm 0.0015$ & $0.91895 \pm 0.00066$ & $1.0240 \pm 0.0018$ \\
Likelihood ID (LID) & $0.9638 \pm 0.0011$ & $0.97813 \pm 0.00036$ & $0.9854 \pm 0.0012$ \\
Overall & $0.6766 \pm 0.0024$ & $0.70975 \pm 0.00093$ & $0.9533 \pm 0.0036$ \\
\hline \hline
\end{tabular}


Table A.23: Low $E_{T}$ electron selection cut efficiencies and scale factors for $0 \mathrm{p}$ data set.

\begin{tabular}{cccc}
\hline \hline Cut & Data Efficiency & MC Efficiency & Scale factor \\
\hline COT hit & $0.9946 \pm 0.0022$ & $0.99736 \pm 0.00026$ & $0.9972 \pm 0.0022$ \\
$z_{0}-z_{\mathrm{pv}}$ & $0.9860 \pm 0.0031$ & $0.99073 \pm 0.00048$ & $0.9953 \pm 0.0031$ \\
$d_{0}$ & $0.9632 \pm 0.0060$ & $0.9461 \pm 0.0011$ & $1.0180 \pm 0.0065$ \\
Silicon hit & $0.9215 \pm 0.0067$ & $0.9136 \pm 0.0014$ & $1.0087 \pm 0.0075$ \\
ISO $_{0.4}^{\text {cal }}$ & $0.6068 \pm 0.0098$ & $0.6600 \pm 0.0024$ & $0.919 \pm 0.015$ \\
Track Quality \& ISO cal & $0.5394 \pm 0.0093$ & $0.5912 \pm 0.0025$ & $0.912 \pm 0.016$ \\
\hline Conversion removal (CR) & $0.9098 \pm 0.0049$ & $0.97935 \pm 0.00094$ & $0.9290 \pm 0.0050$ \\
Likelihood ID (LID) & $0.8703 \pm 0.0062$ & $0.8904 \pm 0.0020$ & $0.9774 \pm 0.0073$ \\
Overall & $0.5394 \pm 0.0093$ & $0.5912 \pm 0.0025$ & $0.912 \pm 0.016$ \\
\hline \hline
\end{tabular}

Table A.24: High $E_{T}$ electron selection cut efficiencies and scale factors for $0 \mathrm{p}$ data set.

\begin{tabular}{cccc}
\hline \hline Cut & Data Efficiency & MC Efficiency & Scale factor \\
\hline COT hit & $0.99486 \pm 0.00047$ & $0.997959 \pm 0.000093$ & $0.99689 \pm 0.00048$ \\
$z_{0}-z_{\mathrm{pv}}$ & $0.98793 \pm 0.00071$ & $0.99223 \pm 0.00018$ & $0.99567 \pm 0.00073$ \\
$d_{0}$ & $0.9596 \pm 0.0013$ & $0.95832 \pm 0.00041$ & $1.0013 \pm 0.0014$ \\
Silicon hit & $0.9111 \pm 0.0016$ & $0.91822 \pm 0.00056$ & $0.9923 \pm 0.0019$ \\
ISO $_{0.4}^{\text {cal }}$ & $0.7559 \pm 0.0023$ & $0.79147 \pm 0.00083$ & $0.9551 \pm 0.0031$ \\
Track Quality \& ISO cal & $0.6737 \pm 0.0025$ & $0.70975 \pm 0.00093$ & $0.9492 \pm 0.0037$ \\
\hline Conversion removal (CR) & $0.8201 \pm 0.0023$ & $0.91895 \pm 0.00066$ & $0.8924 \pm 0.0026$ \\
Likelihood ID (LID) & $0.9617 \pm 0.0012$ & $0.97813 \pm 0.00036$ & $0.9832 \pm 0.0012$ \\
Overall & $0.6737 \pm 0.0025$ & $0.70975 \pm 0.00093$ & $0.9492 \pm 0.0037$ \\
\hline \hline
\end{tabular}




\section{A.3.2 Muon Selection Efficiency and Scale Factor}

Table A.25: Muon selection cut efficiencies and scale factors from CMUP-CMUP for od data set.

\begin{tabular}{cccc}
\hline \hline Cut & Data Efficiency & MC Efficiency & Scale factor \\
\hline COT hit & $0.9777 \pm 0.0038$ & $0.999759 \pm 0.000085$ & $0.9779 \pm 0.0038$ \\
$z_{0}-z_{\mathrm{pv}}$ & $1.00 \pm 0.00$ & $0.99928 \pm 0.00015$ & $1.00072 \pm 0.00015$ \\
$d_{0}$ & $0.9983 \pm 0.0022$ & $0.99836 \pm 0.00020$ & $1.0000 \pm 0.0022$ \\
Silicon hit & $0.9228 \pm 0.0070$ & $0.9359 \pm 0.0013$ & $0.9860 \pm 0.0076$ \\
ISO $_{0.4}^{\text {cal }}$ & $0.8894 \pm 0.0088$ & $0.9215 \pm 0.0015$ & $0.9652 \pm 0.0097$ \\
Track Quality \& ISO 0.4 & $0.836 \pm 0.010$ & $0.8614 \pm 0.0019$ & $0.971 \pm 0.012$ \\
\hline Likelihood ID (LID) & $0.9592 \pm 0.0055$ & $0.9679 \pm 0.0010$ & $0.9911 \pm 0.0058$ \\
\hline Overall & $0.802 \pm 0.011$ & $0.8337 \pm 0.0020$ & $0.962 \pm 0.013$ \\
\hline \hline
\end{tabular}

Table A.26: Muon selection cut efficiencies and scale factors from CMX-CMUP for Od data set.

\begin{tabular}{cccc}
\hline \hline Cut & Data Efficiency & MC Efficiency & Scale factor \\
\hline COT hit & $0.9763 \pm 0.0055$ & $0.99966 \pm 0.00015$ & $0.9767 \pm 0.0055$ \\
$z_{0}-z_{\mathrm{pv}}$ & $0.9988 \pm 0.0023$ & $0.99957 \pm 0.00017$ & $0.9992 \pm 0.0023$ \\
$d_{0}$ & $0.9990 \pm 0.0023$ & $0.99791 \pm 0.00033$ & $1.0011 \pm 0.0023$ \\
Silicon hit & $0.907 \pm 0.011$ & $0.9159 \pm 0.0023$ & $0.990 \pm 0.012$ \\
ISO $_{0.4}^{\text {cal }}$ & $0.895 \pm 0.012$ & $0.9211 \pm 0.0022$ & $0.972 \pm 0.013$ \\
Track Quality \& ISO cal $^{2}$ & $0.824 \pm 0.015$ & $0.8409 \pm 0.0030$ & $0.980 \pm 0.018$ \\
\hline Likelihood ID (LID) & $0.9524 \pm 0.0088$ & $0.9617 \pm 0.0017$ & $0.9903 \pm 0.0093$ \\
\hline Overall & $0.785 \pm 0.016$ & $0.8087 \pm 0.0032$ & $0.971 \pm 0.020$ \\
\hline \hline
\end{tabular}


Table A.27: Muon selection cut efficiencies and scale factors from CMUP-CMX for Od data set.

\begin{tabular}{cccc}
\hline \hline Cut & Data Efficiency & MC Efficiency & Scale factor \\
\hline COT hit & $0.9772 \pm 0.0064$ & $0.99965 \pm 0.00015$ & $0.9776 \pm 0.0064$ \\
$z_{0}-z_{\mathrm{pv}}$ & $1.00 \pm 0.00$ & $0.99924 \pm 0.00023$ & $1.00076 \pm 0.00023$ \\
$d_{0}$ & $0.9991 \pm 0.0027$ & $0.99854 \pm 0.00026$ & $1.0006 \pm 0.0027$ \\
Silicon hit & $0.926 \pm 0.011$ & $0.9582 \pm 0.0017$ & $0.966 \pm 0.011$ \\
ISO $_{0.4}^{\text {cal }}$ & $0.892 \pm 0.013$ & $0.9273 \pm 0.0022$ & $0.962 \pm 0.014$ \\
Track Quality \& ISO ca. $^{\text {cal }}$ & $0.844 \pm 0.015$ & $0.8873 \pm 0.0026$ & $0.951 \pm 0.017$ \\
\hline Likelihood ID (LID) & $0.9796 \pm 0.0061$ & $0.9828 \pm 0.0011$ & $0.9968 \pm 0.0063$ \\
\hline Overall & $0.827 \pm 0.015$ & $0.8720 \pm 0.0028$ & $0.948 \pm 0.018$ \\
\hline \hline
\end{tabular}

Table A.28: Muon selection cut efficiencies and scale factors from CMX-CMX for 0d data set.

\begin{tabular}{cccc}
\hline \hline Cut & Data Efficiency & MC Efficiency & Scale factor \\
\hline COT hit & $0.9813 \pm 0.0066$ & $0.99958 \pm 0.00022$ & $0.9817 \pm 0.0066$ \\
$z_{0}-z_{\mathrm{pv}}$ & $1.00 \pm 0.00$ & $0.99925 \pm 0.00031$ & $1.00075 \pm 0.00031$ \\
$d_{0}$ & $1.00 \pm 0.00$ & $0.99830 \pm 0.00039$ & $1.00170 \pm 0.00039$ \\
Silicon hit $_{\text {ISO }}^{\text {cal }}$ & $0.941 \pm 0.011$ & $0.9481 \pm 0.0025$ & $0.993 \pm 0.012$ \\
Track Quality \& ISO & $0.881 \pm 0.016$ & $0.9266 \pm 0.0029$ & $0.951 \pm 0.018$ \\
\hline Likelihood ID (LID) & $0.851 \pm 0.018$ & $0.8763 \pm 0.0037$ & $0.971 \pm 0.021$ \\
\hline Overall & $0.837 \pm 0.0067$ & $0.9803 \pm 0.0016$ & $1.0032 \pm 0.0070$ \\
\hline \hline
\end{tabular}

Table A.29: Muon selection cut efficiencies and scale factors from CMUP-(CMIOCES or CMIOPES) for Od data set.

\begin{tabular}{cccc}
\hline \hline Cut & Data Efficiency & MC Efficiency & Scale factor \\
\hline COT hit & $0.9965 \pm 0.0048$ & $0.99926 \pm 0.00025$ & $0.9972 \pm 0.0048$ \\
$z_{0}-z_{\mathrm{pv}}$ & $0.9976 \pm 0.0060$ & $0.99901 \pm 0.00029$ & $0.9986 \pm 0.0060$ \\
$d_{0}$ & $1.00 \pm 0.00$ & $0.99884 \pm 0.00031$ & $1.00116 \pm 0.00031$ \\
Silicon hit & $0.972 \pm 0.011$ & $0.9592 \pm 0.0018$ & $1.013 \pm 0.012$ \\
ISO $_{0.4}^{\text {cal }}$ & $0.934 \pm 0.018$ & $0.9308 \pm 0.0023$ & $1.003 \pm 0.019$ \\
Track Quality \& ISO ${ }_{0.4}^{\text {cal }}$ & $0.897 \pm 0.020$ & $0.8930 \pm 0.0028$ & $1.004 \pm 0.022$ \\
\hline Likelihood ID (LID) & $0.713 \pm 0.023$ & $0.6510 \pm 0.0046$ & $1.095 \pm 0.036$ \\
\hline Overall & $0.639 \pm 0.025$ & $0.5813 \pm 0.0045$ & $1.099 \pm 0.044$ \\
\hline \hline
\end{tabular}


Table A.30: Muon selection cut efficiencies and scale factors from CMX-(CMIOCES or CMIOPES) for od data set.

\begin{tabular}{cccc}
\hline \hline Cut & Data Efficiency & MC Efficiency & Scale factor \\
\hline COT hit & $1.00 \pm 0.00$ & $0.99768 \pm 0.00077$ & $1.00232 \pm 0.00077$ \\
$z_{0}-z_{\mathrm{pv}}$ & $1.00 \pm 0.00$ & $0.99897 \pm 0.00051$ & $1.00103 \pm 0.00052$ \\
$d_{0}$ & $1.00 \pm 0.00$ & $0.99923 \pm 0.00045$ & $1.00077 \pm 0.00045$ \\
Silicon hit & $0.943 \pm 0.024$ & $0.9395 \pm 0.0038$ & $1.004 \pm 0.026$ \\
ISO $_{0.4}^{\text {cal }}$ & $0.940 \pm 0.033$ & $0.9292 \pm 0.0041$ & $1.012 \pm 0.036$ \\
Track Quality \& ISO cal $^{\text {cal }}$ & $0.861 \pm 0.037$ & $0.8756 \pm 0.0053$ & $0.984 \pm 0.042$ \\
\hline Likelihood ID (LID) & $0.758 \pm 0.035$ & $0.6755 \pm 0.0080$ & $1.122 \pm 0.053$ \\
\hline Overall & $0.653 \pm 0.041$ & $0.5915 \pm 0.0079$ & $1.104 \pm 0.070$ \\
\hline \hline
\end{tabular}

Table A.31: Muon selection cut efficiencies and scale factors from CMUP-(CMU, CMP, or BMU) for Od data set.

\begin{tabular}{cccc}
\hline \hline Cut & Data Efficiency & MC Efficiency & Scale factor \\
\hline COT hit & $0.9815 \pm 0.0060$ & $0.99901 \pm 0.00027$ & $0.9824 \pm 0.0060$ \\
$z_{0}-z_{\mathrm{pv}}$ & $0.9968 \pm 0.0023$ & $0.99863 \pm 0.00032$ & $0.9981 \pm 0.0023$ \\
$d_{0}$ & $0.9984 \pm 0.0016$ & $0.99863 \pm 0.00032$ & $0.9998 \pm 0.0016$ \\
Silicon hit & $0.935 \pm 0.010$ & $0.9469 \pm 0.0020$ & $0.987 \pm 0.011$ \\
ISO $_{0.4}^{\text {cal }}$ & $0.906 \pm 0.013$ & $0.9317 \pm 0.0022$ & $0.973 \pm 0.014$ \\
Track Quality \& ISO cal $^{\text {cal }}$ & $0.858 \pm 0.015$ & $0.8821 \pm 0.0028$ & $0.973 \pm 0.017$ \\
\hline Likelihood ID (LID) & $0.9756 \pm 0.0067$ & $0.9731 \pm 0.0015$ & $1.0025 \pm 0.0070$ \\
\hline Overall & $0.837 \pm 0.016$ & $0.8584 \pm 0.0030$ & $0.975 \pm 0.019$ \\
\hline \hline
\end{tabular}

Table A.32: Muon selection cut efficiencies and scale factors from CMX-(CMU, CMP, or BMU) for Od data set.

\begin{tabular}{cccc}
\hline \hline Cut & Data Efficiency & MC Efficiency & Scale factor \\
\hline COT hit & $0.9853 \pm 0.0086$ & $0.99983 \pm 0.00017$ & $0.9854 \pm 0.0086$ \\
$z_{0}-z_{\mathrm{pv}}$ & $0.9935 \pm 0.0046$ & $0.99851 \pm 0.00050$ & $0.9949 \pm 0.0046$ \\
$d_{0}$ & $0.9967 \pm 0.0033$ & $0.99901 \pm 0.00041$ & $0.9977 \pm 0.0033$ \\
Silicon hit & $0.917 \pm 0.017$ & $0.9321 \pm 0.0032$ & $0.983 \pm 0.018$ \\
ISO $_{0.4}^{\text {cal }}$ & $0.921 \pm 0.018$ & $0.9391 \pm 0.0031$ & $0.981 \pm 0.020$ \\
Track Quality \& ISO cal $^{\text {cal }}$ & $0.863 \pm 0.022$ & $0.8752 \pm 0.0043$ & $0.986 \pm 0.025$ \\
\hline Likelihood ID (LID) & $0.951 \pm 0.013$ & $0.9777 \pm 0.0020$ & $0.972 \pm 0.014$ \\
\hline Overall & $0.820 \pm 0.024$ & $0.8556 \pm 0.0045$ & $0.959 \pm 0.028$ \\
\hline \hline
\end{tabular}


Table A.33: Muon selection cut efficiencies and scale factors from CMUP-CMUP for Oh data set.

\begin{tabular}{|c|c|c|c|}
\hline Cut & "Data Efficiency & MC Efficiency & Scale factor \\
\hline COT hit & $0.9982 \pm 0.0010$ & $0.99950 \pm 0.00011$ & $0.9987 \pm 0.0010$ \\
\hline$z_{0}-z_{\mathrm{pv}}$ & $0.9987 \pm 0.0012$ & $0.99935 \pm 0.00012$ & $0.9994 \pm 0.0012$ \\
\hline$d_{0}$ & $0.9991 \pm 0.0022$ & $0.99808 \pm 0.00020$ & $1.0010 \pm 0.0022$ \\
\hline Silicon hit & $0.9528 \pm 0.0055$ & $0.9453 \pm 0.0011$ & $1.0080 \pm 0.0059$ \\
\hline $\mathrm{ISO}_{0.4}^{\mathrm{cal}}$ & $0.8801 \pm 0.0085$ & $0.9141 \pm 0.0014$ & $0.9628 \pm 0.0094$ \\
\hline Track Quality \& ISO $_{0.4}^{\text {cal }}$ & $0.8334 \pm 0.0095$ & $0.8628 \pm 0.0017$ & $0.966 \pm 0.011$ \\
\hline Likelihood ID (LID) & $0.9551 \pm 0.0055$ & $0.96602 \pm 0.00094$ & $0.9886 \pm 0.0058$ \\
\hline Overall & $0.796 \pm 0.010$ & $0.8335 \pm 0.0018$ & $0.955 \pm 0.012$ \\
\hline
\end{tabular}

Table A.34: Muon selection cut efficiencies and scale factors from CMX-CMUP for Oh data set.

\begin{tabular}{cccc}
\hline \hline Cut & Data Efficiency & MC Efficiency & Scale factor \\
\hline COT hit & $0.9991 \pm 0.0017$ & $0.99953 \pm 0.00014$ & $0.9996 \pm 0.0017$ \\
$z_{0}-z_{\mathrm{pv}}$ & $1.00 \pm 0.00$ & $0.99958 \pm 0.00013$ & $1.00042 \pm 0.00013$ \\
$d_{0}$ & $0.9992 \pm 0.0029$ & $0.99774 \pm 0.00028$ & $1.0015 \pm 0.0029$ \\
Silicon hit $_{\text {ISO }}^{\text {cal }}$ & $0.9345 \pm 0.0080$ & $0.9286 \pm 0.0016$ & $1.0063 \pm 0.0088$ \\
Track Quality \& ISO & $0.900 \pm 0.010$ & $0.9117 \pm 0.0018$ & $0.988 \pm 0.011$ \\
\hline Likelihood ID (LID) & $0.836 \pm 0.012$ & $0.8454 \pm 0.0023$ & $0.989 \pm 0.014$ \\
\hline Overall & $0.9612 \pm 0.0067$ & $0.9623 \pm 0.0013$ & $0.9988 \pm 0.0071$ \\
\hline \hline
\end{tabular}


Table A.35: Muon selection cut efficiencies and scale factors from CMUP-CMX for Oh data set.

\begin{tabular}{cccc}
\hline \hline Cut & Data Efficiency & MC Efficiency & Scale factor \\
\hline COT hit & $1.00 \pm 0.00$ & $0.99943 \pm 0.00015$ & $1.00057 \pm 0.00015$ \\
$z_{0}-z_{\mathrm{pv}}$ & $0.9982 \pm 0.0019$ & $0.99898 \pm 0.00020$ & $0.9992 \pm 0.0019$ \\
$d_{0}$ & $0.9976 \pm 0.0028$ & $0.99830 \pm 0.00024$ & $0.9993 \pm 0.0029$ \\
Silicon hit & $0.9665 \pm 0.0060$ & $0.9675 \pm 0.0011$ & $0.9989 \pm 0.0063$ \\
ISO $_{0.4}^{\text {cal }}$ & $0.9023 \pm 0.0099$ & $0.9157 \pm 0.0018$ & $0.985 \pm 0.011$ \\
Track Quality \& ISO ca. $^{\text {ca }}$ & $0.870 \pm 0.011$ & $0.8847 \pm 0.0020$ & $0.984 \pm 0.013$ \\
\hline Likelihood ID (LID) & $0.9805 \pm 0.0048$ & $0.98203 \pm 0.00088$ & $0.9985 \pm 0.0050$ \\
\hline Overall & $0.853 \pm 0.012$ & $0.8688 \pm 0.0021$ & $0.982 \pm 0.014$ \\
\hline \hline
\end{tabular}

Table A.36: Muon selection cut efficiencies and scale factors from CMX-CMX for Oh data set.

\begin{tabular}{cccc}
\hline \hline Cut & Data Efficiency & MC Efficiency & Scale factor \\
\hline COT hit & $0.9956 \pm 0.0025$ & $0.99928 \pm 0.00022$ & $0.9963 \pm 0.0025$ \\
$z_{0}-z_{\mathrm{pv}}$ & $1.00 \pm 0.00$ & $0.99933 \pm 0.00021$ & $1.00067 \pm 0.00021$ \\
$d_{0}$ & $1.00 \pm 0.00$ & $0.99822 \pm 0.00032$ & $1.00178 \pm 0.00032$ \\
Silicon hit & $0.9665 \pm 0.0069$ & $0.9626 \pm 0.0016$ & $1.0040 \pm 0.0073$ \\
ISO $_{0.4}^{\text {cal }}$ & $0.895 \pm 0.012$ & $0.9122 \pm 0.0023$ & $0.981 \pm 0.014$ \\
Track Quality \& ISO cal & $0.865 \pm 0.014$ & $0.8771 \pm 0.0027$ & $0.987 \pm 0.016$ \\
\hline Likelihood ID (LID) & $0.9773 \pm 0.0067$ & $0.9795 \pm 0.0012$ & $0.9978 \pm 0.0069$ \\
\hline Overall & $0.846 \pm 0.015$ & $0.8591 \pm 0.0028$ & $0.984 \pm 0.017$ \\
\hline \hline
\end{tabular}

Table A.37: Muon selection cut efficiencies and scale factors from CMUP-(CMIOCES or CMIOPES) for Oh data set.

\begin{tabular}{cccc}
\hline \hline Cut & Data Efficiency & MC Efficiency & Scale factor \\
\hline COT hit & $0.9934 \pm 0.0060$ & $0.99846 \pm 0.00041$ & $0.9949 \pm 0.0061$ \\
$z_{0}-z_{\mathrm{pv}}$ & $1.00 \pm 0.00$ & $0.99934 \pm 0.00027$ & $1.00066 \pm 0.00027$ \\
$d_{0}$ & $1.00 \pm 0.00$ & $0.99901 \pm 0.00033$ & $1.00099 \pm 0.00033$ \\
Silicon hit & $0.980 \pm 0.012$ & $0.9696 \pm 0.0018$ & $1.011 \pm 0.013$ \\
ISO $_{0.4}^{\text {cal }}$ & $0.943 \pm 0.023$ & $0.9322 \pm 0.0026$ & $1.012 \pm 0.025$ \\
Track Quality \& ISO ${ }_{0.4}^{\text {cal }}$ & $0.936 \pm 0.025$ & $0.9044 \pm 0.0031$ & $1.035 \pm 0.028$ \\
\hline Likelihood ID (LID) & $0.662 \pm 0.025$ & $0.6213 \pm 0.0054$ & $1.065 \pm 0.042$ \\
\hline Overall & $0.619 \pm 0.029$ & $0.5620 \pm 0.0052$ & $1.102 \pm 0.052$ \\
\hline \hline
\end{tabular}


Table A.38: Muon selection cut efficiencies and scale factors from CMX-(CMIOCES or CMIOPES) for Oh data set.

\begin{tabular}{cccc}
\hline \hline Cut & Data Efficiency & MC Efficiency & Scale factor \\
\hline COT hit & $0.9958 \pm 0.0042$ & $0.99709 \pm 0.00078$ & $0.9987 \pm 0.0043$ \\
$z_{0}-z_{\mathrm{pv}}$ & $0.9915 \pm 0.0060$ & $0.99938 \pm 0.00036$ & $0.9922 \pm 0.0060$ \\
$d_{0}$ & $0.9939 \pm 0.0085$ & $0.99979 \pm 0.00021$ & $0.9941 \pm 0.0085$ \\
Silicon hit & $0.928 \pm 0.017$ & $0.9622 \pm 0.0027$ & $0.965 \pm 0.018$ \\
ISO $_{0.4}^{\text {cal }}$ & $0.930 \pm 0.028$ & $0.9297 \pm 0.0037$ & $1.001 \pm 0.030$ \\
Track Quality \& ISO cal & $0.880 \pm 0.030$ & $0.8961 \pm 0.0044$ & $0.982 \pm 0.034$ \\
\hline Likelihood ID (LID) & $0.678 \pm 0.032$ & $0.6260 \pm 0.0074$ & $1.083 \pm 0.053$ \\
\hline Overall & $0.596 \pm 0.035$ & $0.5610 \pm 0.0071$ & $1.063 \pm 0.064$ \\
\hline \hline
\end{tabular}

Table A.39: Muon selection cut efficiencies and scale factors from CMUP-(CMU, CMP, or BMU) for Oh data set.

\begin{tabular}{cccc}
\hline \hline Cut & Data Efficiency & MC Efficiency & Scale factor \\
\hline COT hit & $0.9960 \pm 0.0023$ & $0.99919 \pm 0.00022$ & $0.9968 \pm 0.0023$ \\
$z_{0}-z_{\mathrm{pv}}$ & $0.9928 \pm 0.0037$ & $0.99875 \pm 0.00028$ & $0.9940 \pm 0.0038$ \\
$d_{0}$ & $0.9994 \pm 0.0023$ & $0.99856 \pm 0.00030$ & $1.0008 \pm 0.0023$ \\
Silicon hit & $0.9496 \pm 0.0080$ & $0.9537 \pm 0.0017$ & $0.9957 \pm 0.0085$ \\
ISO $_{0.4}^{\text {cal }}$ & $0.895 \pm 0.011$ & $0.9263 \pm 0.0021$ & $0.967 \pm 0.013$ \\
Track Quality \& ISO 0.4 & $0.854 \pm 0.013$ & $0.8829 \pm 0.0025$ & $0.967 \pm 0.015$ \\
\hline Likelihood ID (LID) & $0.9581 \pm 0.0079$ & $0.9726 \pm 0.0014$ & $0.9851 \pm 0.0082$ \\
\hline Overall & $0.818 \pm 0.014$ & $0.8587 \pm 0.0028$ & $0.953 \pm 0.017$ \\
\hline \hline
\end{tabular}

Table A.40: Muon selection cut efficiencies and scale factors from CMX-(CMU, CMP, or BMU) for Oh data set.

\begin{tabular}{cccc}
\hline \hline Cut & Data Efficiency & MC Efficiency & Scale factor \\
\hline COT hit & $1.00 \pm 0.00$ & $0.99957 \pm 0.00021$ & $1.00043 \pm 0.00021$ \\
$z_{0}-z_{\mathrm{pv}}$ & $1.00 \pm 0.00$ & $0.99925 \pm 0.00028$ & $1.00075 \pm 0.00028$ \\
$d_{0}$ & $1.00 \pm 0.00$ & $0.99936 \pm 0.00026$ & $1.00064 \pm 0.00026$ \\
Silicon hit & $0.953 \pm 0.012$ & $0.9407 \pm 0.0024$ & $1.013 \pm 0.013$ \\
ISO $_{0.4}^{\text {cal }}$ & $0.930 \pm 0.016$ & $0.9292 \pm 0.0027$ & $1.001 \pm 0.017$ \\
Track Quality \& ISO 0.4 & $0.875 \pm 0.019$ & $0.8751 \pm 0.0034$ & $1.000 \pm 0.022$ \\
\hline Likelihood ID (LID) & $0.9756 \pm 0.0080$ & $0.9716 \pm 0.0018$ & $1.0042 \pm 0.0085$ \\
\hline Overall & $0.854 \pm 0.020$ & $0.8502 \pm 0.0037$ & $1.004 \pm 0.023$ \\
\hline \hline
\end{tabular}


Table A.41: Muon selection cut efficiencies and scale factors from CMUP-CMUP for Oi data set.

\begin{tabular}{cccc}
\hline \hline Cut & Data Efficiency & MC Efficiency & Scale factor \\
\hline COT hit & $0.9970 \pm 0.0012$ & $0.999429 \pm 0.000095$ & $0.9976 \pm 0.0012$ \\
$z_{0}-z_{\mathrm{pv}}$ & $0.9944 \pm 0.0017$ & $0.99933 \pm 0.00010$ & $0.9950 \pm 0.0017$ \\
$d_{0}$ & $0.9948 \pm 0.0021$ & $0.99807 \pm 0.00017$ & $0.9968 \pm 0.0022$ \\
Silicon hit $_{\text {ISO }}^{\text {cal }}$ & $0.9451 \pm 0.0047$ & $0.94435 \pm 0.00091$ & $1.0007 \pm 0.0051$ \\
Track Quality \& ISO cal & $0.8751 \pm 0.0070$ & $0.9041 \pm 0.0012$ & $0.9679 \pm 0.0078$ \\
\hline Likelihood ID (LID) & $0.9649 \pm 0.0040$ & $0.96625 \pm 0.00078$ & $0.9986 \pm 0.0042$ \\
\hline Overall & $0.7931 \pm 0.0083$ & $0.8240 \pm 0.0015$ & $0.963 \pm 0.010$ \\
\hline \hline
\end{tabular}

Table A.42: Muon selection cut efficiencies and scale factors from CMX-CMUP for 0i data set.

\begin{tabular}{cccc}
\hline \hline Cut & Data Efficiency & MC Efficiency & Scale factor \\
\hline COT hit & $0.9953 \pm 0.0018$ & $0.99958 \pm 0.00010$ & $0.9958 \pm 0.0018$ \\
$z_{0}-z_{\mathrm{pv}}$ & $0.9960 \pm 0.0016$ & $0.99908 \pm 0.00015$ & $0.9969 \pm 0.0016$ \\
$d_{0}$ & $0.9947 \pm 0.0023$ & $0.99751 \pm 0.00026$ & $0.9972 \pm 0.0023$ \\
Silicon hit & $0.9334 \pm 0.0065$ & $0.9305 \pm 0.0013$ & $1.0031 \pm 0.0071$ \\
ISO $_{0.4}^{\text {cal }}$ & $0.8797 \pm 0.0089$ & $0.9056 \pm 0.0015$ & $0.9714 \pm 0.0100$ \\
Track Quality \& ISO ${ }_{0.4}^{\text {cal }}$ & $0.825 \pm 0.010$ & $0.8407 \pm 0.0019$ & $0.981 \pm 0.012$ \\
\hline Likelihood ID (LID) & $0.9573 \pm 0.0059$ & $0.9634 \pm 0.0010$ & $0.9936 \pm 0.0062$ \\
\hline Overall & $0.790 \pm 0.011$ & $0.8100 \pm 0.0020$ & $0.975 \pm 0.014$ \\
\hline \hline
\end{tabular}


Table A.43: Muon selection cut efficiencies and scale factors from CMUP-CMX for 0i data set.

\begin{tabular}{cccc}
\hline \hline Cut & Data Efficiency & MC Efficiency & Scale factor \\
\hline COT hit & $0.9964 \pm 0.0019$ & $0.99917 \pm 0.00015$ & $0.9972 \pm 0.0019$ \\
$z_{0}-z_{\mathrm{pv}}$ & $0.9935 \pm 0.0024$ & $0.99931 \pm 0.00014$ & $0.9942 \pm 0.0024$ \\
$d_{0}$ & $0.9950 \pm 0.0024$ & $0.99847 \pm 0.00020$ & $0.9965 \pm 0.0024$ \\
Silicon hit & $0.9612 \pm 0.0054$ & $0.96439 \pm 0.00096$ & $0.9967 \pm 0.0057$ \\
ISO $_{0.4}^{\text {cal }}$ & $0.9056 \pm 0.0089$ & $0.9117 \pm 0.0015$ & $0.9932 \pm 0.0099$ \\
Track Quality \& ISO ca. $^{\text {cal }}$ & $0.8710 \pm 0.0098$ & $0.8781 \pm 0.0017$ & $0.992 \pm 0.011$ \\
\hline Likelihood ID (LID) & $0.9793 \pm 0.0041$ & $0.98208 \pm 0.00073$ & $0.9972 \pm 0.0042$ \\
\hline Overall & $0.853 \pm 0.010$ & $0.8623 \pm 0.0018$ & $0.989 \pm 0.012$ \\
\hline \hline
\end{tabular}

Table A.44: Muon selection cut efficiencies and scale factors from CMX-CMX for 0i data set.

\begin{tabular}{cccc}
\hline \hline Cut & Data Efficiency & MC Efficiency & Scale factor \\
\hline COT hit & $1.00 \pm 0.00$ & $0.99927 \pm 0.00018$ & $1.00073 \pm 0.00018$ \\
$z_{0}-z_{\mathrm{pv}}$ & $1.00 \pm 0.00$ & $0.99897 \pm 0.00021$ & $1.00104 \pm 0.00021$ \\
$d_{0}$ & $0.9974 \pm 0.0034$ & $0.99853 \pm 0.00025$ & $0.9988 \pm 0.0034$ \\
Silicon hit $^{\text {cal }}$ & $0.9721 \pm 0.0057$ & $0.9592 \pm 0.0013$ & $1.0134 \pm 0.0061$ \\
ISO $_{0.4}$ & $0.878 \pm 0.011$ & $0.9109 \pm 0.0019$ & $0.964 \pm 0.012$ \\
Track Quality \& ISO cal $^{\text {cal }}$ & $0.850 \pm 0.011$ & $0.8724 \pm 0.0022$ & $0.974 \pm 0.013$ \\
\hline Likelihood ID (LID) & $0.9626 \pm 0.0063$ & $0.98042 \pm 0.00097$ & $0.9819 \pm 0.0065$ \\
\hline Overall & $0.818 \pm 0.012$ & $0.8553 \pm 0.0023$ & $0.957 \pm 0.015$ \\
\hline \hline
\end{tabular}

Table A.45: Muon selection cut efficiencies and scale factors from CMUP-(CMIOCES or CMIOPES) for 0i data set.

\begin{tabular}{cccc}
\hline \hline Cut & Data Efficiency & MC Efficiency & Scale factor \\
\hline COT hit & $1.00 \pm 0.00$ & $0.99788 \pm 0.00039$ & $1.00213 \pm 0.00040$ \\
$z_{0}-z_{\mathrm{pv}}$ & $1.00 \pm 0.00$ & $0.99876 \pm 0.00030$ & $1.00125 \pm 0.00030$ \\
$d_{0}$ & $1.00 \pm 0.00$ & $0.99854 \pm 0.00033$ & $1.00147 \pm 0.00033$ \\
Silicon hit & $0.9669 \pm 0.0091$ & $0.9645 \pm 0.0016$ & $1.0024 \pm 0.0095$ \\
ISO $_{0.4}^{\text {cal }}$ & $0.863 \pm 0.018$ & $0.9253 \pm 0.0022$ & $0.933 \pm 0.019$ \\
Track Quality \& ISO cal $^{\text {cal }}$ & $0.834 \pm 0.018$ & $0.8932 \pm 0.0026$ & $0.934 \pm 0.021$ \\
\hline Likelihood ID (LID) & $0.664 \pm 0.021$ & $0.6229 \pm 0.0044$ & $1.066 \pm 0.034$ \\
\hline Overall & $0.554 \pm 0.021$ & $0.5564 \pm 0.0042$ & $0.996 \pm 0.039$ \\
\hline \hline
\end{tabular}


Table A.46: Muon selection cut efficiencies and scale factors from CMX-(CMIOCES or CMIOPES) for 0i data set.

\begin{tabular}{cccc}
\hline \hline Cut & Data Efficiency & MC Efficiency & Scale factor \\
\hline COT hit & $1.00 \pm 0.00$ & $0.99767 \pm 0.00056$ & $1.00234 \pm 0.00057$ \\
$z_{0}-z_{\mathrm{pv}}$ & $1.00 \pm 0.00$ & $0.99918 \pm 0.00034$ & $1.00082 \pm 0.00034$ \\
$d_{0}$ & $1.00 \pm 0.00$ & $0.99890 \pm 0.00039$ & $1.00110 \pm 0.00039$ \\
Silicon hit & $0.966 \pm 0.015$ & $0.9641 \pm 0.0022$ & $1.002 \pm 0.016$ \\
ISO $_{0.4}^{\text {cal }}$ & $0.882 \pm 0.025$ & $0.9238 \pm 0.0031$ & $0.955 \pm 0.027$ \\
Track Quality \& ISO cal & $0.838 \pm 0.026$ & $0.8916 \pm 0.0036$ & $0.940 \pm 0.030$ \\
\hline Likelihood ID (LID) & $0.722 \pm 0.027$ & $0.6420 \pm 0.0059$ & $1.124 \pm 0.044$ \\
\hline Overall & $0.605 \pm 0.030$ & $0.5724 \pm 0.0058$ & $1.056 \pm 0.053$ \\
\hline \hline
\end{tabular}

Table A.47: Muon selection cut efficiencies and scale factors from CMUP-(CMU, CMP, or BMU) for 0i data set.

\begin{tabular}{cccc}
\hline \hline Cut & Data Efficiency & MC Efficiency & Scale factor \\
\hline COT hit & $0.9982 \pm 0.0027$ & $0.99913 \pm 0.00019$ & $0.9991 \pm 0.0027$ \\
$z_{0}-z_{\mathrm{pv}}$ & $1.00 \pm 0.00$ & $0.99896 \pm 0.00021$ & $1.00104 \pm 0.00021$ \\
$d_{0}$ & $1.00 \pm 0.00$ & $0.99843 \pm 0.00026$ & $1.00158 \pm 0.00026$ \\
Silicon hit & $0.9611 \pm 0.0066$ & $0.9512 \pm 0.0014$ & $1.0105 \pm 0.0071$ \\
ISO $_{0.4}^{\text {cal }}$ & $0.896 \pm 0.011$ & $0.9184 \pm 0.0018$ & $0.975 \pm 0.012$ \\
Track Quality \& ISO ${ }_{0.4}^{\text {cal }}$ & $0.861 \pm 0.012$ & $0.8736 \pm 0.0021$ & $0.985 \pm 0.014$ \\
\hline Likelihood ID (LID) & $0.9624 \pm 0.0063$ & $0.9756 \pm 0.0011$ & $0.9865 \pm 0.0066$ \\
\hline Overall & $0.828 \pm 0.012$ & $0.8523 \pm 0.0023$ & $0.972 \pm 0.015$ \\
\hline \hline
\end{tabular}

Table A.48: Muon selection cut efficiencies and scale factors from CMX-(CMU, CMP, or BMU) for 0i data set.

\begin{tabular}{cccc}
\hline \hline Cut & Data Efficiency & MC Efficiency & Scale factor \\
\hline COT hit & $0.9956 \pm 0.0025$ & $0.99865 \pm 0.00031$ & $0.9969 \pm 0.0026$ \\
$z_{0}-z_{\mathrm{pv}}$ & $0.9956 \pm 0.0025$ & $0.99794 \pm 0.00038$ & $0.9976 \pm 0.0026$ \\
$d_{0}$ & $0.9941 \pm 0.0029$ & $0.99879 \pm 0.00029$ & $0.9953 \pm 0.0030$ \\
Silicon hit & $0.9500 \pm 0.0084$ & $0.9427 \pm 0.0020$ & $1.0077 \pm 0.0091$ \\
ISO $_{0.4}^{\text {cal }}$ & $0.909 \pm 0.012$ & $0.9183 \pm 0.0023$ & $0.990 \pm 0.013$ \\
Track Quality \& ISO cal $^{\text {cal }}$ & $0.868 \pm 0.014$ & $0.8657 \pm 0.0029$ & $1.002 \pm 0.016$ \\
\hline Likelihood ID (LID) & $0.9610 \pm 0.0080$ & $0.9731 \pm 0.0015$ & $0.9876 \pm 0.0083$ \\
\hline Overall & $0.834 \pm 0.015$ & $0.8424 \pm 0.0031$ & $0.990 \pm 0.018$ \\
\hline \hline
\end{tabular}


Table A.49: Muon selection cut efficiencies and scale factors from CMUP-CMUP for $0 \mathrm{j}$ data set.

\begin{tabular}{cccc}
\hline \hline Cut & Data Efficiency & MC Efficiency & Scale factor \\
\hline COT hit & $0.9963 \pm 0.0013$ & $0.99890 \pm 0.00011$ & $0.9974 \pm 0.0013$ \\
$z_{0}-z_{\mathrm{pv}}$ & $0.9948 \pm 0.0014$ & $0.99887 \pm 0.00011$ & $0.9960 \pm 0.0014$ \\
$d_{0}$ & $0.9969 \pm 0.0014$ & $0.99800 \pm 0.00015$ & $0.9989 \pm 0.0014$ \\
Silicon hit & $0.9455 \pm 0.0040$ & $0.93513 \pm 0.00080$ & $1.0111 \pm 0.0044$ \\
ISO $_{0.4}^{\text {cal }}$ & $0.8617 \pm 0.0063$ & $0.8877 \pm 0.0010$ & $0.9706 \pm 0.0072$ \\
Track Quality \& ISO cal $^{\text {cal }}$ & $0.8143 \pm 0.0070$ & $0.8292 \pm 0.0012$ & $0.9819 \pm 0.0085$ \\
\hline Likelihood ID (LID) & $0.9588 \pm 0.0038$ & $0.96745 \pm 0.00064$ & $0.9911 \pm 0.0040$ \\
\hline Overall & $0.7808 \pm 0.0074$ & $0.8022 \pm 0.0013$ & $0.9732 \pm 0.0093$ \\
\hline \hline
\end{tabular}

Table A.50: Muon selection cut efficiencies and scale factors from CMX-CMUP for $0 \mathrm{j}$ data set.

\begin{tabular}{cccc}
\hline \hline Cut & Data Efficiency & MC Efficiency & Scale factor \\
\hline COT hit & $0.9972 \pm 0.0011$ & $0.99903 \pm 0.00013$ & $0.9982 \pm 0.0011$ \\
$z_{0}-z_{\mathrm{pv}}$ & $0.9955 \pm 0.0017$ & $0.99900 \pm 0.00013$ & $0.9965 \pm 0.0017$ \\
$d_{0}$ & $0.9928 \pm 0.0023$ & $0.99753 \pm 0.00021$ & $0.9953 \pm 0.0023$ \\
Silicon hit & $0.9330 \pm 0.0055$ & $0.9199 \pm 0.0011$ & $1.0142 \pm 0.0061$ \\
ISO $_{0.4}^{\text {cal }}$ & $0.8538 \pm 0.0080$ & $0.8875 \pm 0.0013$ & $0.9620 \pm 0.0091$ \\
Track Quality \& ISO cal $^{\text {cal }}$ & $0.7930 \pm 0.0090$ & $0.8146 \pm 0.0016$ & $0.973 \pm 0.011$ \\
\hline Likelihood ID (LID) & $0.9555 \pm 0.0050$ & $0.96416 \pm 0.00086$ & $0.9910 \pm 0.0052$ \\
\hline Overall & $0.7577 \pm 0.0095$ & $0.7854 \pm 0.0017$ & $0.965 \pm 0.012$ \\
\hline \hline
\end{tabular}


Table A.51: Muon selection cut efficiencies and scale factors from CMUP-CMX for 0j data set.

\begin{tabular}{cccc}
\hline \hline Cut & Data Efficiency & MC Efficiency & Scale factor \\
\hline COT hit & $0.9976 \pm 0.0013$ & $0.99896 \pm 0.00014$ & $0.9986 \pm 0.0013$ \\
$z_{0}-z_{\mathrm{pv}}$ & $0.9934 \pm 0.0022$ & $0.99878 \pm 0.00015$ & $0.9946 \pm 0.0022$ \\
$d_{0}$ & $0.9924 \pm 0.0024$ & $0.99847 \pm 0.00016$ & $0.9940 \pm 0.0024$ \\
Silicon hit & $0.9506 \pm 0.0049$ & $0.95732 \pm 0.00085$ & $0.9930 \pm 0.0052$ \\
ISO $_{0.4}^{\text {cal }}$ & $0.8677 \pm 0.0080$ & $0.9020 \pm 0.0012$ & $0.9619 \pm 0.0090$ \\
Track Quality \& ISO ca. $^{\text {cal }}$ & $0.8284 \pm 0.0088$ & $0.8629 \pm 0.0014$ & $0.960 \pm 0.010$ \\
\hline Likelihood ID (LID) & $0.9726 \pm 0.0040$ & $0.98181 \pm 0.00060$ & $0.9906 \pm 0.0042$ \\
\hline Overall & $0.8057 \pm 0.0092$ & $0.8472 \pm 0.0015$ & $0.951 \pm 0.011$ \\
\hline \hline
\end{tabular}

Table A.52: Muon selection cut efficiencies and scale factors from CMX-CMX for $0 \mathrm{j}$ data set.

\begin{tabular}{cccc}
\hline \hline Cut & Data Efficiency & MC Efficiency & Scale factor \\
\hline COT hit & $0.9952 \pm 0.0018$ & $0.99884 \pm 0.00018$ & $0.9963 \pm 0.0018$ \\
$z_{0}-z_{\mathrm{pv}}$ & $0.9938 \pm 0.0021$ & $0.99864 \pm 0.00020$ & $0.9951 \pm 0.0021$ \\
$d_{0}$ & $0.9953 \pm 0.0023$ & $0.99860 \pm 0.00020$ & $0.9966 \pm 0.0023$ \\
Silicon hit & $0.9615 \pm 0.0052$ & $0.9545 \pm 0.0011$ & $1.0073 \pm 0.0056$ \\
ISO $_{0.4}^{\text {cal }}$ & $0.8509 \pm 0.0097$ & $0.8971 \pm 0.0016$ & $0.948 \pm 0.011$ \\
Track Quality \& ISO cal $^{2}$ & $0.818 \pm 0.010$ & $0.8558 \pm 0.0019$ & $0.956 \pm 0.012$ \\
\hline Likelihood ID (LID) & $0.9807 \pm 0.0043$ & $0.98141 \pm 0.00078$ & $0.9993 \pm 0.0045$ \\
\hline Overall & $0.803 \pm 0.011$ & $0.8399 \pm 0.0020$ & $0.956 \pm 0.013$ \\
\hline \hline
\end{tabular}

Table A.53: Muon selection cut efficiencies and scale factors from CMUP-(CMIOCES or CMIOPES) for $0 j$ data set.

\begin{tabular}{cccc}
\hline \hline Cut & Data Efficiency & MC Efficiency & Scale factor \\
\hline COT hit & $0.9941 \pm 0.0033$ & $0.99889 \pm 0.00024$ & $0.9952 \pm 0.0033$ \\
$z_{0}-z_{\mathrm{pv}}$ & $0.9952 \pm 0.0066$ & $0.99874 \pm 0.00025$ & $0.9965 \pm 0.0066$ \\
$d_{0}$ & $0.9982 \pm 0.0072$ & $0.99884 \pm 0.00024$ & $0.9993 \pm 0.0073$ \\
Silicon hit & $0.9622 \pm 0.0086$ & $0.9608 \pm 0.0014$ & $1.0015 \pm 0.0091$ \\
ISO $_{0.4}^{\text {cal }}$ & $0.844 \pm 0.017$ & $0.9173 \pm 0.0020$ & $0.920 \pm 0.018$ \\
Track Quality \& ISO cal $^{\text {cal }}$ & $0.817 \pm 0.017$ & $0.8817 \pm 0.0023$ & $0.927 \pm 0.020$ \\
\hline Likelihood ID (LID) & $0.623 \pm 0.019$ & $0.6112 \pm 0.0037$ & $1.019 \pm 0.032$ \\
\hline Overall & $0.509 \pm 0.019$ & $0.5389 \pm 0.0035$ & $0.944 \pm 0.036$ \\
\hline \hline
\end{tabular}


Table A.54: Muon selection cut efficiencies and scale factors from CMX-(CMIOCES or CMIOPES) for $0 \mathrm{j}$ data set.

\begin{tabular}{cccc}
\hline \hline Cut & Data Efficiency & MC Efficiency & Scale factor \\
\hline COT hit & $0.9971 \pm 0.0049$ & $0.99790 \pm 0.00044$ & $0.9992 \pm 0.0049$ \\
$z_{0}-z_{\mathrm{pv}}$ & $0.9971 \pm 0.0075$ & $0.99845 \pm 0.00038$ & $0.9986 \pm 0.0076$ \\
$d_{0}$ & $1.00 \pm 0.00$ & $0.99872 \pm 0.00034$ & $1.00128 \pm 0.00034$ \\
Silicon hit & $0.968 \pm 0.012$ & $0.9612 \pm 0.0018$ & $1.007 \pm 0.013$ \\
ISO $_{0.4}^{\text {cal }}$ & $0.856 \pm 0.022$ & $0.9125 \pm 0.0027$ & $0.939 \pm 0.024$ \\
Track Quality \& ISO ${ }_{0.4}^{\text {cal }}$ & $0.814 \pm 0.023$ & $0.8774 \pm 0.0031$ & $0.928 \pm 0.026$ \\
\hline Likelihood ID (LID) & $0.664 \pm 0.023$ & $0.6398 \pm 0.0049$ & $1.038 \pm 0.037$ \\
\hline Overall & $0.541 \pm 0.024$ & $0.5614 \pm 0.0047$ & $0.963 \pm 0.044$ \\
\hline \hline
\end{tabular}

Table A.55: Muon selection cut efficiencies and scale factors from CMUP-(CMU, CMP, or $\mathrm{BMU}$ ) for $0 \mathrm{j}$ data set.

\begin{tabular}{cccc}
\hline \hline Cut & Data Efficiency & MC Efficiency & Scale factor \\
\hline COT hit & $0.9939 \pm 0.0020$ & $0.99834 \pm 0.00022$ & $0.9956 \pm 0.0020$ \\
$z_{0}-z_{\mathrm{pv}}$ & $0.9943 \pm 0.0024$ & $0.99851 \pm 0.00020$ & $0.9957 \pm 0.0024$ \\
$d_{0}$ & $0.9976 \pm 0.0019$ & $0.99812 \pm 0.00023$ & $0.9995 \pm 0.0020$ \\
Silicon hit & $0.9436 \pm 0.0062$ & $0.9482 \pm 0.0012$ & $0.9952 \pm 0.0066$ \\
ISO $_{0.4}^{\text {cal }}$ & $0.8632 \pm 0.0096$ & $0.9053 \pm 0.0016$ & $0.953 \pm 0.011$ \\
Track Quality \& ISO cal & $0.818 \pm 0.011$ & $0.8593 \pm 0.0018$ & $0.952 \pm 0.013$ \\
\hline Likelihood ID (LID) & $0.9680 \pm 0.0052$ & $0.97195 \pm 0.00094$ & $0.9960 \pm 0.0054$ \\
\hline Overall & $0.792 \pm 0.011$ & $0.8352 \pm 0.0020$ & $0.948 \pm 0.013$ \\
\hline \hline
\end{tabular}

Table A.56: Muon selection cut efficiencies and scale factors from CMX-(CMU, CMP, or $\mathrm{BMU}$ ) for $0 \mathrm{j}$ data set.

\begin{tabular}{cccc}
\hline \hline Cut & Data Efficiency & MC Efficiency & Scale factor \\
\hline COT hit & $0.9972 \pm 0.0030$ & $0.99883 \pm 0.00023$ & $0.9983 \pm 0.0030$ \\
$z_{0}-z_{\mathrm{pv}}$ & $0.9923 \pm 0.0034$ & $0.99869 \pm 0.00025$ & $0.9936 \pm 0.0034$ \\
$d_{0}$ & $0.9932 \pm 0.0028$ & $0.99878 \pm 0.00024$ & $0.9944 \pm 0.0028$ \\
Silicon hit & $0.9162 \pm 0.0096$ & $0.9401 \pm 0.0016$ & $0.975 \pm 0.010$ \\
ISO $_{0.4}^{\text {cal }}$ & $0.849 \pm 0.013$ & $0.9062 \pm 0.0020$ & $0.937 \pm 0.014$ \\
Track Quality \& ISO ${ }_{0.4}^{\text {cal }}$ & $0.773 \pm 0.015$ & $0.8518 \pm 0.0024$ & $0.907 \pm 0.017$ \\
\hline Likelihood ID (LID) & $0.9587 \pm 0.0076$ & $0.9730 \pm 0.0012$ & $0.9853 \pm 0.0079$ \\
\hline Overall & $0.741 \pm 0.015$ & $0.8287 \pm 0.0026$ & $0.894 \pm 0.019$ \\
\hline \hline
\end{tabular}


Table A.57: Muon selection cut efficiencies and scale factors from CMUP-CMUP for Ok data set.

\begin{tabular}{cccc}
\hline \hline Cut & Data Efficiency & MC Efficiency & Scale factor \\
\hline COT hit & $0.9947 \pm 0.0017$ & $0.99844 \pm 0.00014$ & $0.9962 \pm 0.0017$ \\
$z_{0}-z_{\mathrm{pv}}$ & $0.9883 \pm 0.0025$ & $0.99855 \pm 0.00014$ & $0.9897 \pm 0.0025$ \\
$d_{0}$ & $0.9921 \pm 0.0022$ & $0.99771 \pm 0.00017$ & $0.9944 \pm 0.0022$ \\
Silicon hit & $0.9394 \pm 0.0056$ & $0.93969 \pm 0.00086$ & $0.9996 \pm 0.0060$ \\
ISO $_{0.4}^{\text {cal }}$ & $0.8489 \pm 0.0088$ & $0.8728 \pm 0.0012$ & $0.973 \pm 0.010$ \\
Track Quality \& ISO ca. $^{\text {ca }}$ & $0.7993 \pm 0.0097$ & $0.8197 \pm 0.0014$ & $0.975 \pm 0.012$ \\
\hline Likelihood ID (LID) & $0.9553 \pm 0.0053$ & $0.96797 \pm 0.00070$ & $0.9869 \pm 0.0056$ \\
\hline Overall & $0.764 \pm 0.010$ & $0.7935 \pm 0.0015$ & $0.962 \pm 0.013$ \\
\hline \hline
\end{tabular}

Table A.58: Muon selection cut efficiencies and scale factors from CMX-CMUP for Ok data set.

\begin{tabular}{cccc}
\hline \hline Cut & Data Efficiency & MC Efficiency & Scale factor \\
\hline COT hit & $1.00 \pm 0.00$ & $0.99864 \pm 0.00017$ & $1.00136 \pm 0.00017$ \\
$z_{0}-z_{\mathrm{pv}}$ & $0.9935 \pm 0.0028$ & $0.99856 \pm 0.00017$ & $0.9950 \pm 0.0028$ \\
$d_{0}$ & $0.9936 \pm 0.0028$ & $0.99718 \pm 0.00024$ & $0.9964 \pm 0.0028$ \\
Silicon hit $_{\text {ISO }}^{\text {cal }}$ & $0.9375 \pm 0.0076$ & $0.9281 \pm 0.0012$ & $1.0102 \pm 0.0083$ \\
Track Quality \& ISO & $0.830 \pm 0.012$ & $0.8725 \pm 0.0015$ & $0.951 \pm 0.014$ \\
\hline Likelihood ID (LID) & $0.774 \pm 0.013$ & $0.8086 \pm 0.0018$ & $0.957 \pm 0.016$ \\
\hline Overall & $0.7304 \pm 0.0076$ & $0.96392 \pm 0.00094$ & $0.9859 \pm 0.0079$ \\
\hline \hline
\end{tabular}


Table A.59: Muon selection cut efficiencies and scale factors from CMUP-CMX for Ok data set.

\begin{tabular}{cccc}
\hline \hline Cut & Data Efficiency & MC Efficiency & Scale factor \\
\hline COT hit & $0.9950 \pm 0.0026$ & $0.99804 \pm 0.00021$ & $0.9970 \pm 0.0027$ \\
$z_{0}-z_{\mathrm{pv}}$ & $0.9961 \pm 0.0028$ & $0.99802 \pm 0.00021$ & $0.9981 \pm 0.0028$ \\
$d_{0}$ & $0.9931 \pm 0.0030$ & $0.99794 \pm 0.00021$ & $0.9952 \pm 0.0030$ \\
Silicon hit & $0.9610 \pm 0.0063$ & $0.96088 \pm 0.00090$ & $1.0001 \pm 0.0066$ \\
ISO $_{0.4}^{\text {cal }}$ & $0.868 \pm 0.012$ & $0.8878 \pm 0.0015$ & $0.978 \pm 0.013$ \\
Track Quality \& ISO & $0.836 \pm 0.013$ & $0.8533 \pm 0.0016$ & $0.980 \pm 0.015$ \\
\hline Likelihood ID (LID) & $0.9741 \pm 0.0062$ & $0.98298 \pm 0.00065$ & $0.9910 \pm 0.0064$ \\
\hline Overall & $0.815 \pm 0.013$ & $0.8388 \pm 0.0017$ & $0.971 \pm 0.016$ \\
\hline \hline
\end{tabular}

Table A.60: Muon selection cut efficiencies and scale factors from CMX-CMX for 0k data set.

\begin{tabular}{cccc}
\hline \hline Cut & Data Efficiency & MC Efficiency & Scale factor \\
\hline COT hit & $0.9965 \pm 0.0028$ & $0.99818 \pm 0.00025$ & $0.9983 \pm 0.0028$ \\
$z_{0}-z_{\mathrm{pv}}$ & $1.00 \pm 0.00$ & $0.99865 \pm 0.00022$ & $1.00135 \pm 0.00022$ \\
$d_{0}$ & $0.9990 \pm 0.0022$ & $0.99832 \pm 0.00023$ & $1.0007 \pm 0.0022$ \\
Silicon hit $_{\text {ISO }}^{\text {cal }}$ & $0.9566 \pm 0.0076$ & $0.9578 \pm 0.0012$ & $0.9988 \pm 0.0081$ \\
Track Quality \& ISO & $0.844 \pm 0.013$ & $0.8806 \pm 0.0019$ & $0.958 \pm 0.015$ \\
Likelihood ID (LID) & $0.811 \pm 0.014$ & $0.8431 \pm 0.0021$ & $0.962 \pm 0.017$ \\
\hline Overall & $0.784 \pm 0.015$ & $0.8251 \pm 0.0022$ & $0.950 \pm 0.018$ \\
\hline \hline
\end{tabular}

Table A.61: Muon selection cut efficiencies and scale factors from CMUP-(CMIOCES or CMIOPES) for 0k data set.

\begin{tabular}{cccc}
\hline \hline Cut & Data Efficiency & MC Efficiency & Scale factor \\
\hline COT hit & $1.0000 \pm 0.0042$ & $0.99825 \pm 0.00032$ & $1.0017 \pm 0.0042$ \\
$z_{0}-z_{\mathrm{pv}}$ & $1.00 \pm 0.00$ & $0.99813 \pm 0.00033$ & $1.00187 \pm 0.00034$ \\
$d_{0}$ & $1.0000 \pm 0.0092$ & $0.99837 \pm 0.00031$ & $1.0016 \pm 0.0092$ \\
Silicon hit & $0.959 \pm 0.013$ & $0.9638 \pm 0.0015$ & $0.995 \pm 0.014$ \\
ISO $_{0.4}^{\text {cal }}$ & $0.841 \pm 0.021$ & $0.9023 \pm 0.0023$ & $0.932 \pm 0.024$ \\
Track Quality \& ISO cal $^{\text {cal }}$ & $0.818 \pm 0.023$ & $0.8701 \pm 0.0026$ & $0.940 \pm 0.026$ \\
\hline Likelihood ID (LID) & $0.581 \pm 0.026$ & $0.6139 \pm 0.0040$ & $0.946 \pm 0.043$ \\
\hline Overall & $0.475 \pm 0.025$ & $0.5341 \pm 0.0039$ & $0.889 \pm 0.047$ \\
\hline \hline
\end{tabular}


Table A.62: Muon selection cut efficiencies and scale factors from CMX-(CMIOCES or CMIOPES) for 0k data set.

\begin{tabular}{cccc}
\hline \hline Cut & Data Efficiency & MC Efficiency & Scale factor \\
\hline COT hit & $1.00 \pm 0.00$ & $0.99752 \pm 0.00053$ & $1.00248 \pm 0.00053$ \\
$z_{0}-z_{\mathrm{pv}}$ & $1.00 \pm 0.00$ & $0.99797 \pm 0.00048$ & $1.00203 \pm 0.00048$ \\
$d_{0}$ & $1.00 \pm 0.00$ & $0.99854 \pm 0.00041$ & $1.00147 \pm 0.00041$ \\
Silicon hit & $0.975 \pm 0.016$ & $0.9595 \pm 0.0021$ & $1.017 \pm 0.017$ \\
ISO $_{0.4}^{\text {cal }}$ & $0.967 \pm 0.032$ & $0.8934 \pm 0.0033$ & $1.083 \pm 0.036$ \\
Track Quality \& ISO cal $^{\text {cal }}$ & $0.926 \pm 0.033$ & $0.8575 \pm 0.0037$ & $1.080 \pm 0.039$ \\
\hline Likelihood ID (LID) & $0.699 \pm 0.031$ & $0.6255 \pm 0.0055$ & $1.118 \pm 0.050$ \\
\hline Overall & $0.648 \pm 0.037$ & $0.5364 \pm 0.0053$ & $1.207 \pm 0.069$ \\
\hline \hline
\end{tabular}

Table A.63: Muon selection cut efficiencies and scale factors from CMUP-(CMU, CMP, or BMU) for $0 \mathrm{k}$ data set.

\begin{tabular}{|c|c|c|c|}
\hline Cut & Data Efficiency & MC Efficiency & Scale factor \\
\hline COT hit & $0.9939 \pm 0.0027$ & $0.99809 \pm 0.00026$ & $0.9958 \pm 0.0027$ \\
\hline$z_{0}-z_{\mathrm{pv}}$ & $0.9903 \pm 0.0034$ & $0.99809 \pm 0.00026$ & $0.9922 \pm 0.0034$ \\
\hline$d_{0}$ & $0.9951 \pm 0.0024$ & $0.99802 \pm 0.00026$ & $0.9971 \pm 0.0024$ \\
\hline Silicon hit & $0.9429 \pm 0.0081$ & $0.9501 \pm 0.0013$ & $0.9925 \pm 0.0086$ \\
\hline $\mathrm{ISO}_{0.4}^{\mathrm{cal}}$ & $0.868 \pm 0.012$ & $0.8883 \pm 0.0018$ & $0.977 \pm 0.014$ \\
\hline Track Quality \& $\mathrm{ISO}_{0.4}^{\text {cal }}$ & $0.829 \pm 0.013$ & $0.8439 \pm 0.0021$ & $0.982 \pm 0.016$ \\
\hline Likelihood ID (LID) & $0.9707 \pm 0.0065$ & $0.9737 \pm 0.0010$ & $0.9969 \pm 0.0067$ \\
\hline Overall & $0.804 \pm 0.014$ & $0.8217 \pm 0.0022$ & $0.979 \pm 0.017$ \\
\hline
\end{tabular}

Table A.64: Muon selection cut efficiencies and scale factors from CMX-(CMU, CMP, or BMU) for $0 \mathrm{k}$ data set.

\begin{tabular}{cccc}
\hline \hline Cut & Data Efficiency & MC Efficiency & Scale factor \\
\hline COT hit & $0.9924 \pm 0.0038$ & $0.99806 \pm 0.00033$ & $0.9944 \pm 0.0038$ \\
$z_{0}-z_{\mathrm{pv}}$ & $0.9887 \pm 0.0058$ & $0.99749 \pm 0.00038$ & $0.9911 \pm 0.0058$ \\
$d_{0}$ & $0.9981 \pm 0.0040$ & $0.99818 \pm 0.00032$ & $0.9999 \pm 0.0040$ \\
Silicon hit & $0.930 \pm 0.012$ & $0.9442 \pm 0.0017$ & $0.985 \pm 0.013$ \\
ISO $_{0.4}^{\text {cal }}$ & $0.856 \pm 0.016$ & $0.8877 \pm 0.0024$ & $0.965 \pm 0.018$ \\
Track Quality \& ISO cal $^{\text {cal }}$ & $0.788 \pm 0.018$ & $0.8392 \pm 0.0028$ & $0.939 \pm 0.022$ \\
\hline Likelihood ID (LID) & $0.954 \pm 0.010$ & $0.9746 \pm 0.0013$ & $0.979 \pm 0.011$ \\
\hline Overall & $0.752 \pm 0.019$ & $0.8179 \pm 0.0029$ & $0.920 \pm 0.024$ \\
\hline \hline
\end{tabular}


Table A.65: Muon selection cut efficiencies and scale factors from CMUP-CMUP for Om data set.

\begin{tabular}{cccc}
\hline \hline Cut & Data Efficiency & MC Efficiency & Scale factor \\
\hline COT hit & $0.99616 \pm 0.00074$ & $0.99844 \pm 0.00014$ & $0.99772 \pm 0.00076$ \\
$z_{0}-z_{\mathrm{pv}}$ & $0.99440 \pm 0.00092$ & $0.99855 \pm 0.00014$ & $0.99584 \pm 0.00093$ \\
$d_{0}$ & $0.9931 \pm 0.0011$ & $0.99771 \pm 0.00017$ & $0.9954 \pm 0.0011$ \\
Silicon hit $^{\text {cal }}$ & $0.9181 \pm 0.0029$ & $0.93969 \pm 0.00086$ & $0.9770 \pm 0.0032$ \\
ISO $_{0.4}$ & $0.8182 \pm 0.0041$ & $0.8728 \pm 0.0012$ & $0.9374 \pm 0.0049$ \\
Track Quality \& ISO ${ }_{0.4}^{\text {cal }}$ & $0.7498 \pm 0.0045$ & $0.8197 \pm 0.0014$ & $0.9148 \pm 0.0057$ \\
\hline Likelihood ID (LID) & $0.9578 \pm 0.0024$ & $0.96797 \pm 0.00070$ & $0.9895 \pm 0.0025$ \\
\hline Overall & $0.7182 \pm 0.0047$ & $0.7935 \pm 0.0015$ & $0.9052 \pm 0.0061$ \\
\hline \hline
\end{tabular}

Table A.66: Muon selection cut efficiencies and scale factors from CMX-CMUP for 0m data set.

\begin{tabular}{cccc}
\hline \hline Cut & Data Efficiency & MC Efficiency & Scale factor \\
\hline COT hit & $0.9934 \pm 0.0010$ & $0.99864 \pm 0.00017$ & $0.9948 \pm 0.0010$ \\
$z_{0}-z_{\mathrm{pv}}$ & $0.9920 \pm 0.0013$ & $0.99856 \pm 0.00017$ & $0.9934 \pm 0.0013$ \\
$d_{0}$ & $0.9938 \pm 0.0012$ & $0.99718 \pm 0.00024$ & $0.9966 \pm 0.0012$ \\
Silicon hit & $0.9044 \pm 0.0037$ & $0.9281 \pm 0.0012$ & $0.9745 \pm 0.0042$ \\
ISO $_{0.4}^{\text {cal }}$ & $0.8174 \pm 0.0050$ & $0.8725 \pm 0.0015$ & $0.9368 \pm 0.0059$ \\
Track Quality \& ISO ${ }_{0.4}^{\text {cal }}$ & $0.7410 \pm 0.0056$ & $0.8086 \pm 0.0018$ & $0.9165 \pm 0.0072$ \\
\hline Likelihood ID (LID) & $0.9508 \pm 0.0031$ & $0.96392 \pm 0.00094$ & $0.9864 \pm 0.0034$ \\
\hline Overall & $0.7046 \pm 0.0058$ & $0.7794 \pm 0.0019$ & $0.9040 \pm 0.0077$ \\
\hline \hline
\end{tabular}


Table A.67: Muon selection cut efficiencies and scale factors from CMUP-CMX for 0m data set.

\begin{tabular}{cccc}
\hline \hline Cut & Data Efficiency & MC Efficiency & Scale factor \\
\hline COT hit & $0.9952 \pm 0.0011$ & $0.99804 \pm 0.00021$ & $0.9971 \pm 0.0011$ \\
$z_{0}-z_{\mathrm{pv}}$ & $0.9911 \pm 0.0013$ & $0.99802 \pm 0.00021$ & $0.9931 \pm 0.0014$ \\
$d_{0}$ & $0.9941 \pm 0.0013$ & $0.99794 \pm 0.00021$ & $0.9962 \pm 0.0014$ \\
Silicon hit & $0.9472 \pm 0.0031$ & $0.96088 \pm 0.00090$ & $0.9857 \pm 0.0033$ \\
ISO $_{0.4}^{\text {cal }}$ & $0.8315 \pm 0.0052$ & $0.8878 \pm 0.0015$ & $0.9365 \pm 0.0060$ \\
Track Quality \& ISO ca. $^{\text {ca }}$ & $0.7879 \pm 0.0056$ & $0.8533 \pm 0.0016$ & $0.9234 \pm 0.0068$ \\
\hline Likelihood ID (LID) & $0.9730 \pm 0.0024$ & $0.98298 \pm 0.00065$ & $0.9899 \pm 0.0025$ \\
\hline Overall & $0.7667 \pm 0.0057$ & $0.8388 \pm 0.0017$ & $0.9140 \pm 0.0071$ \\
\hline \hline
\end{tabular}

Table A.68: Muon selection cut efficiencies and scale factors from CMX-CMX for 0m data set.

\begin{tabular}{cccc}
\hline \hline Cut & Data Efficiency & MC Efficiency & Scale factor \\
\hline COT hit & $0.9937 \pm 0.0013$ & $0.99818 \pm 0.00025$ & $0.9955 \pm 0.0014$ \\
$z_{0}-z_{\mathrm{pv}}$ & $0.9898 \pm 0.0016$ & $0.99865 \pm 0.00022$ & $0.9912 \pm 0.0016$ \\
$d_{0}$ & $0.9956 \pm 0.0013$ & $0.99832 \pm 0.00023$ & $0.9973 \pm 0.0014$ \\
Silicon hit & $0.9448 \pm 0.0035$ & $0.9578 \pm 0.0012$ & $0.9864 \pm 0.0039$ \\
ISO cal $_{0.4}^{\text {cal }}$ & $0.8220 \pm 0.0060$ & $0.8806 \pm 0.0019$ & $0.9335 \pm 0.0071$ \\
Track Quality \& ISO 0.4 & $0.7798 \pm 0.0064$ & $0.8431 \pm 0.0021$ & $0.9250 \pm 0.0080$ \\
\hline Likelihood ID (LID) & $0.9754 \pm 0.0027$ & $0.97869 \pm 0.00092$ & $0.9966 \pm 0.0029$ \\
\hline Overall & $0.7606 \pm 0.0066$ & $0.8251 \pm 0.0022$ & $0.9218 \pm 0.0084$ \\
\hline \hline
\end{tabular}

Table A.69: Muon selection cut efficiencies and scale factors from CMUP-(CMIOCES or CMIOPES) for Om data set.

\begin{tabular}{cccc}
\hline \hline Cut & Data Efficiency & MC Efficiency & Scale factor \\
\hline COT hit & $1.00 \pm 0.00$ & $0.99825 \pm 0.00032$ & $1.00175 \pm 0.00033$ \\
$z_{0}-z_{\mathrm{pv}}$ & $0.9987 \pm 0.0030$ & $0.99813 \pm 0.00033$ & $1.0006 \pm 0.0030$ \\
$d_{0}$ & $1.00 \pm 0.00$ & $0.99837 \pm 0.00031$ & $1.00163 \pm 0.00031$ \\
Silicon hit & $0.9737 \pm 0.0049$ & $0.9638 \pm 0.0015$ & $1.0103 \pm 0.0053$ \\
ISO $_{0.4}^{\text {cal }}$ & $0.858 \pm 0.010$ & $0.9023 \pm 0.0023$ & $0.951 \pm 0.011$ \\
Track Quality \& ISO ${ }_{0.4}^{\text {cal }}$ & $0.827 \pm 0.010$ & $0.8701 \pm 0.0026$ & $0.950 \pm 0.012$ \\
\hline Likelihood ID (LID) & $0.627 \pm 0.011$ & $0.6139 \pm 0.0040$ & $1.022 \pm 0.019$ \\
\hline Overall & $0.519 \pm 0.011$ & $0.5341 \pm 0.0039$ & $0.971 \pm 0.022$ \\
\hline \hline
\end{tabular}


Table A.70: Muon selection cut efficiencies and scale factors from CMX-(CMIOCES or CMIOPES) for Om data set.

\begin{tabular}{cccc}
\hline \hline Cut & Data Efficiency & MC Efficiency & Scale factor \\
\hline COT hit & $0.9976 \pm 0.0029$ & $0.99752 \pm 0.00053$ & $1.0001 \pm 0.0030$ \\
$z_{0}-z_{\mathrm{pv}}$ & $0.9916 \pm 0.0042$ & $0.99797 \pm 0.00048$ & $0.9936 \pm 0.0043$ \\
$d_{0}$ & $0.9911 \pm 0.0045$ & $0.99854 \pm 0.00041$ & $0.9926 \pm 0.0046$ \\
Silicon hit & $0.9491 \pm 0.0069$ & $0.9595 \pm 0.0021$ & $0.9892 \pm 0.0075$ \\
ISO $_{0.4}^{\text {cal }}$ & $0.847 \pm 0.012$ & $0.8934 \pm 0.0033$ & $0.949 \pm 0.014$ \\
Track Quality \& ISO cal $^{\text {cal }}$ & $0.799 \pm 0.013$ & $0.8575 \pm 0.0037$ & $0.932 \pm 0.015$ \\
\hline Likelihood ID (LID) & $0.673 \pm 0.014$ & $0.6255 \pm 0.0055$ & $1.075 \pm 0.024$ \\
\hline Overall & $0.538 \pm 0.014$ & $0.5364 \pm 0.0053$ & $1.002 \pm 0.027$ \\
\hline \hline
\end{tabular}

Table A.71: Muon selection cut efficiencies and scale factors from CMUP-(CMU, CMP, or BMU) for 0m data set.

\begin{tabular}{cccc}
\hline \hline Cut & Data Efficiency & MC Efficiency & Scale factor \\
\hline COT hit & $0.9935 \pm 0.0015$ & $0.99809 \pm 0.00026$ & $0.9954 \pm 0.0015$ \\
$z_{0}-z_{\mathrm{pv}}$ & $0.9909 \pm 0.0017$ & $0.99809 \pm 0.00026$ & $0.9928 \pm 0.0017$ \\
$d_{0}$ & $0.9955 \pm 0.0014$ & $0.99802 \pm 0.00026$ & $0.9974 \pm 0.0014$ \\
Silicon hit & $0.9428 \pm 0.0036$ & $0.9501 \pm 0.0013$ & $0.9923 \pm 0.0040$ \\
ISO $_{0.4}^{\text {cal }}$ & $0.8381 \pm 0.0057$ & $0.8883 \pm 0.0018$ & $0.9435 \pm 0.0068$ \\
Track Quality \& ISO ${ }_{0.4}^{\text {cal }}$ & $0.7906 \pm 0.0062$ & $0.8439 \pm 0.0021$ & $0.9369 \pm 0.0078$ \\
\hline Likelihood ID (LID) & $0.9609 \pm 0.0032$ & $0.9737 \pm 0.0010$ & $0.9869 \pm 0.0035$ \\
\hline Overall & $0.7598 \pm 0.0065$ & $0.8217 \pm 0.0022$ & $0.9246 \pm 0.0083$ \\
\hline \hline
\end{tabular}

Table A.72: Muon selection cut efficiencies and scale factors from CMX-(CMU, CMP, or BMU) for $0 \mathrm{~m}$ data set.

\begin{tabular}{|c|c|c|c|}
\hline Cut & Data Efficiency & MC Efficiency & Scale factor \\
\hline COT hit & $0.9866 \pm 0.0022$ & $0.99806 \pm 0.00033$ & $0.9885 \pm 0.0023$ \\
\hline$z_{0}-z_{\mathrm{pv}}$ & $0.9877 \pm 0.0023$ & $0.99749 \pm 0.00038$ & $0.9902 \pm 0.0023$ \\
\hline$d_{0}$ & $0.9969 \pm 0.0014$ & $0.99818 \pm 0.00032$ & $0.9987 \pm 0.0015$ \\
\hline Silicon hit & $0.9292 \pm 0.0048$ & $0.9442 \pm 0.0017$ & $0.9841 \pm 0.0054$ \\
\hline $\mathrm{ISO}_{0.4}^{\mathrm{cal}}$ & $0.8248 \pm 0.0071$ & $0.8877 \pm 0.0024$ & $0.9292 \pm 0.0084$ \\
\hline Track Quality \& $\mathrm{ISO}_{0.4}^{\mathrm{cal}}$ & $0.7756 \pm 0.0078$ & $0.8392 \pm 0.0028$ & $0.9242 \pm 0.0097$ \\
\hline Likelihood ID (LID) & $0.9590 \pm 0.0041$ & $0.9746 \pm 0.0013$ & $0.9840 \pm 0.0044$ \\
\hline Overall & $0.7438 \pm 0.0081$ & $0.8179 \pm 0.0029$ & $0.909 \pm 0.010$ \\
\hline
\end{tabular}


Table A.73: Muon selection cut efficiencies and scale factors from CMUP-CMUP for Op data set.

\begin{tabular}{cccc}
\hline \hline Cut & Data Efficiency & MC Efficiency & Scale factor \\
\hline COT hit & $0.99415 \pm 0.00087$ & $0.99844 \pm 0.00014$ & $0.99571 \pm 0.00088$ \\
$z_{0}-z_{\text {pv }}$ & $0.9917 \pm 0.0011$ & $0.99855 \pm 0.00014$ & $0.9931 \pm 0.0011$ \\
$d_{0}$ & $0.9936 \pm 0.0012$ & $0.99771 \pm 0.00017$ & $0.9959 \pm 0.0012$ \\
Silicon hit & $0.9344 \pm 0.0027$ & $0.93969 \pm 0.00086$ & $0.9943 \pm 0.0030$ \\
ISO $_{0.4}^{\text {cal }}$ & $0.8047 \pm 0.0044$ & $0.8728 \pm 0.0012$ & $0.9220 \pm 0.0052$ \\
Track Quality \& ISO cal & $0.7529 \pm 0.0047$ & $0.8197 \pm 0.0014$ & $0.9185 \pm 0.0060$ \\
\hline Likelihood ID (LID) & $0.9560 \pm 0.0025$ & $0.96797 \pm 0.00070$ & $0.9877 \pm 0.0027$ \\
\hline Overall & $0.7198 \pm 0.0049$ & $0.7935 \pm 0.0015$ & $0.9072 \pm 0.0064$ \\
\hline \hline
\end{tabular}

Table A.74: Muon selection cut efficiencies and scale factors from CMX-CMUP for Op data set.

\begin{tabular}{cccc}
\hline \hline Cut & Data Efficiency & MC Efficiency & Scale factor \\
\hline COT hit & $0.9929 \pm 0.0011$ & $0.99864 \pm 0.00017$ & $0.9943 \pm 0.0012$ \\
$z_{0}-z_{\text {pv }}$ & $0.9880 \pm 0.0015$ & $0.99856 \pm 0.00017$ & $0.9894 \pm 0.0015$ \\
$d_{0}$ & $0.9921 \pm 0.0016$ & $0.99718 \pm 0.00024$ & $0.9949 \pm 0.0016$ \\
Silicon hit & $0.9132 \pm 0.0038$ & $0.9281 \pm 0.0012$ & $0.9840 \pm 0.0043$ \\
ISO $_{0.4}^{\text {cal }}$ & $0.7958 \pm 0.0056$ & $0.8725 \pm 0.0015$ & $0.9120 \pm 0.0066$ \\
Track Quality \& ISO cal $_{0.4}$ & $0.7267 \pm 0.0061$ & $0.8086 \pm 0.0018$ & $0.8988 \pm 0.0078$ \\
\hline Likelihood ID (LID) & $0.9568 \pm 0.0032$ & $0.96392 \pm 0.00094$ & $0.9926 \pm 0.0035$ \\
\hline Overall & $0.6953 \pm 0.0063$ & $0.7794 \pm 0.0019$ & $0.8921 \pm 0.0083$ \\
\hline \hline
\end{tabular}


Table A.75: Muon selection cut efficiencies and scale factors from CMUP-CMX for 0p data set.

\begin{tabular}{cccc}
\hline \hline Cut & Data Efficiency & MC Efficiency & Scale factor \\
\hline COT hit & $0.9944 \pm 0.0013$ & $0.99804 \pm 0.00021$ & $0.9963 \pm 0.0013$ \\
$z_{0}-z_{\mathrm{pv}}$ & $0.9905 \pm 0.0016$ & $0.99802 \pm 0.00021$ & $0.9925 \pm 0.0016$ \\
$d_{0}$ & $0.9917 \pm 0.0017$ & $0.99794 \pm 0.00021$ & $0.9937 \pm 0.0017$ \\
Silicon hit & $0.9515 \pm 0.0031$ & $0.96088 \pm 0.00090$ & $0.9903 \pm 0.0033$ \\
ISO $_{0.4}^{\text {cal }}$ & $0.8227 \pm 0.0055$ & $0.8878 \pm 0.0015$ & $0.9266 \pm 0.0064$ \\
Track Quality \& ISO ca. $^{\text {ca }}$ & $0.7845 \pm 0.0059$ & $0.8533 \pm 0.0016$ & $0.9194 \pm 0.0071$ \\
\hline Likelihood ID (LID) & $0.9749 \pm 0.0026$ & $0.98298 \pm 0.00065$ & $0.9918 \pm 0.0027$ \\
\hline Overall & $0.7649 \pm 0.0061$ & $0.8388 \pm 0.0017$ & $0.9119 \pm 0.0075$ \\
\hline \hline
\end{tabular}

Table A.76: Muon selection cut efficiencies and scale factors from CMX-CMX for op data set.

\begin{tabular}{cccc}
\hline \hline Cut & Data Efficiency & MC Efficiency & Scale factor \\
\hline COT hit & $0.9947 \pm 0.0013$ & $0.99818 \pm 0.00025$ & $0.9965 \pm 0.0013$ \\
$z_{0}-z_{\mathrm{pv}}$ & $0.9907 \pm 0.0016$ & $0.99865 \pm 0.00022$ & $0.9920 \pm 0.0016$ \\
$d_{0}$ & $0.9934 \pm 0.0015$ & $0.99832 \pm 0.00023$ & $0.9950 \pm 0.0016$ \\
Silicon hit & $0.9388 \pm 0.0037$ & $0.9578 \pm 0.0012$ & $0.9802 \pm 0.0041$ \\
ISO cal $_{0.4}^{\text {cal }}$ & $0.8211 \pm 0.0061$ & $0.8806 \pm 0.0019$ & $0.9325 \pm 0.0072$ \\
Track Quality \& ISO 0.4 & $0.7737 \pm 0.0066$ & $0.8431 \pm 0.0021$ & $0.9177 \pm 0.0082$ \\
\hline Likelihood ID (LID) & $0.9733 \pm 0.0029$ & $0.97869 \pm 0.00092$ & $0.9944 \pm 0.0031$ \\
\hline Overall & $0.7530 \pm 0.0068$ & $0.8251 \pm 0.0022$ & $0.9126 \pm 0.0086$ \\
\hline \hline
\end{tabular}

Table A.77: Muon selection cut efficiencies and scale factors from CMUP-(CMIOCES or CMIOPES) for Op data set.

\begin{tabular}{cccc}
\hline \hline Cut & Data Efficiency & MC Efficiency & Scale factor \\
\hline COT hit & $1.00 \pm 0.00$ & $0.99825 \pm 0.00032$ & $1.00175 \pm 0.00033$ \\
$z_{0}-z_{\mathrm{pv}}$ & $0.9981 \pm 0.0036$ & $0.99813 \pm 0.00033$ & $0.9999 \pm 0.0036$ \\
$d_{0}$ & $0.9955 \pm 0.0043$ & $0.99837 \pm 0.00031$ & $0.9972 \pm 0.0043$ \\
Silicon hit & $0.9565 \pm 0.0055$ & $0.9638 \pm 0.0015$ & $0.9924 \pm 0.0059$ \\
ISO $_{0.4}^{\text {cal }}$ & $0.835 \pm 0.011$ & $0.9023 \pm 0.0023$ & $0.926 \pm 0.012$ \\
Track Quality \& ISO ${ }_{0.4}^{\text {cal }}$ & $0.795 \pm 0.011$ & $0.8701 \pm 0.0026$ & $0.914 \pm 0.013$ \\
\hline Likelihood ID (LID) & $0.631 \pm 0.011$ & $0.6139 \pm 0.0040$ & $1.027 \pm 0.020$ \\
\hline Overall & $0.501 \pm 0.011$ & $0.5341 \pm 0.0039$ & $0.939 \pm 0.022$ \\
\hline \hline
\end{tabular}


Table A.78: Muon selection cut efficiencies and scale factors from CMX-(CMIOCES or CMIOPES) for Op data set.

\begin{tabular}{cccc}
\hline \hline Cut & Data Efficiency & MC Efficiency & Scale factor \\
\hline COT hit & $1.00 \pm 0.00$ & $0.99752 \pm 0.00053$ & $1.00248 \pm 0.00053$ \\
$z_{0}-z_{\mathrm{pv}}$ & $0.9894 \pm 0.0040$ & $0.99797 \pm 0.00048$ & $0.9914 \pm 0.0040$ \\
$d_{0}$ & $0.9949 \pm 0.0037$ & $0.99854 \pm 0.00041$ & $0.9964 \pm 0.0037$ \\
Silicon hit & $0.9436 \pm 0.0076$ & $0.9595 \pm 0.0021$ & $0.9835 \pm 0.0082$ \\
ISO $_{0.4}^{\text {cal }}$ & $0.811 \pm 0.013$ & $0.8934 \pm 0.0033$ & $0.908 \pm 0.015$ \\
Track Quality \& ISO cal & $0.761 \pm 0.014$ & $0.8575 \pm 0.0037$ & $0.888 \pm 0.016$ \\
\hline Likelihood ID (LID) & $0.661 \pm 0.015$ & $0.6255 \pm 0.0055$ & $1.056 \pm 0.025$ \\
\hline Overall & $0.503 \pm 0.014$ & $0.5364 \pm 0.0053$ & $0.938 \pm 0.028$ \\
\hline \hline
\end{tabular}

Table A.79: Muon selection cut efficiencies and scale factors from CMUP-(CMU, CMP, or BMU) for $0 p$ data set.

\begin{tabular}{|c|c|c|c|}
\hline Cut & Data Efficiency & MC Efficiency & Scale factor \\
\hline COT hit & $0.9927 \pm 0.0014$ & $0.99809 \pm 0.00026$ & $0.9946 \pm 0.0014$ \\
\hline$z_{0}-z_{\mathrm{pv}}$ & $0.9906 \pm 0.0017$ & $0.99809 \pm 0.00026$ & $0.9925 \pm 0.0017$ \\
\hline$d_{0}$ & $0.9963 \pm 0.0012$ & $0.99802 \pm 0.00026$ & $0.9983 \pm 0.0012$ \\
\hline Silicon hit & $0.9385 \pm 0.0037$ & $0.9501 \pm 0.0013$ & $0.9878 \pm 0.0041$ \\
\hline $\mathrm{ISO}_{0.4}^{\mathrm{cal}}$ & $0.8187 \pm 0.0059$ & $0.8883 \pm 0.0018$ & $0.9217 \pm 0.0069$ \\
\hline Track Quality \& $\mathrm{ISO}_{0.4}^{\mathrm{cal}}$ & $0.7701 \pm 0.0064$ & $0.8439 \pm 0.0021$ & $0.9126 \pm 0.0080$ \\
\hline Likelihood ID (LID) & $0.9540 \pm 0.0036$ & $0.9737 \pm 0.0010$ & $0.9798 \pm 0.0038$ \\
\hline Overall & $0.7347 \pm 0.0067$ & $0.8217 \pm 0.0022$ & $0.8941 \pm 0.0086$ \\
\hline
\end{tabular}

Table A.80: Muon selection cut efficiencies and scale factors from CMX-(CMU, CMP, or BMU) for $0 \mathrm{p}$ data set.

\begin{tabular}{cccc}
\hline \hline Cut & Data Efficiency & MC Efficiency & Scale factor \\
\hline COT hit & $0.9917 \pm 0.0018$ & $0.99806 \pm 0.00033$ & $0.9936 \pm 0.0019$ \\
$z_{0}-z_{\mathrm{pv}}$ & $0.9902 \pm 0.0020$ & $0.99749 \pm 0.00038$ & $0.9927 \pm 0.0020$ \\
$d_{0}$ & $0.9958 \pm 0.0015$ & $0.99818 \pm 0.00032$ & $0.9976 \pm 0.0015$ \\
Silicon hit & $0.9274 \pm 0.0050$ & $0.9442 \pm 0.0017$ & $0.9822 \pm 0.0056$ \\
ISO $_{0.4}^{\text {cal }}$ & $0.8310 \pm 0.0074$ & $0.8877 \pm 0.0024$ & $0.9361 \pm 0.0087$ \\
Track Quality \& ISO ${ }_{0.4}^{\text {cal }}$ & $0.7750 \pm 0.0082$ & $0.8392 \pm 0.0028$ & $0.924 \pm 0.010$ \\
\hline Likelihood ID (LID) & $0.9632 \pm 0.0041$ & $0.9746 \pm 0.0013$ & $0.9883 \pm 0.0044$ \\
\hline Overall & $0.7465 \pm 0.0085$ & $0.8179 \pm 0.0029$ & $0.913 \pm 0.011$ \\
\hline \hline
\end{tabular}




\section{A.3.3 Muon Reconstruction Efficiency and Scale Factor}

Table A.81: Muon reconstruction efficiencies and scale factors in CMUP-triggered events for Od data set. (AR : Arch, MS : Miniskirt, KS : Keystone)

\begin{tabular}{cccc}
\hline \hline Stub & Data Efficiency & MC Efficiency & Scale factor \\
\hline CMUP & $0.737 \pm 0.010$ & $0.7619 \pm 0.0021$ & $0.968 \pm 0.014$ \\
CMX & $0.9850 \pm 0.0057$ & $0.99743 \pm 0.00043$ & $0.9875 \pm 0.0057$ \\
CMX (AR) & $0.9850 \pm 0.0057$ & $0.99750 \pm 0.00043$ & $0.9875 \pm 0.0057$ \\
CMX (KS) & NA & NA & NA \\
CMX (MS) & NA & NA & NA \\
\hline \hline
\end{tabular}

Table A.82: Muon reconstruction efficiencies and scale factors in CMX-triggered events for Od data set. (AR : Arch, MS : Miniskirt, KS : Keystone)

\begin{tabular}{cccc}
\hline \hline Stub & Data Efficiency & MC Efficiency & Scale factor \\
\hline CMUP & $0.739 \pm 0.015$ & $0.7562 \pm 0.0032$ & $0.977 \pm 0.020$ \\
CMX & $0.9902 \pm 0.0061$ & $0.99772 \pm 0.00055$ & $0.9925 \pm 0.0062$ \\
CMX (AR) & $0.9902 \pm 0.0061$ & $0.99780 \pm 0.00055$ & $0.9924 \pm 0.0062$ \\
CMX (KS) & NA & NA & NA \\
CMX (MS) & NA & NA & NA \\
\hline \hline
\end{tabular}

Table A.83: Muon reconstruction efficiencies and scale factors in CMUP-triggered events for Oh data set. (AR : Arch, MS : Miniskirt, KS : Keystone)

\begin{tabular}{cccc}
\hline \hline Stub & Data Efficiency & MC Efficiency & Scale factor \\
\hline CMUP & $0.7153 \pm 0.0098$ & $0.7608 \pm 0.0019$ & $0.940 \pm 0.013$ \\
CMX & $0.9872 \pm 0.0058$ & $0.99542 \pm 0.00045$ & $0.9917 \pm 0.0058$ \\
CMX (AR) & $0.9953 \pm 0.0061$ & $0.99790 \pm 0.00035$ & $0.9974 \pm 0.0061$ \\
CMX (KS) & $0.977 \pm 0.022$ & $0.9863 \pm 0.0034$ & $0.991 \pm 0.023$ \\
CMX (MS) & $0.955 \pm 0.018$ & $0.9888 \pm 0.0016$ & $0.965 \pm 0.018$ \\
\hline \hline
\end{tabular}


Table A.84: Muon reconstruction efficiencies and scale factors in CMX-triggered events for $\mathrm{Oh}$ data set. (AR : Arch, MS : Miniskirt, KS : Keystone)

\begin{tabular}{cccc}
\hline \hline Stub & Data Efficiency & MC Efficiency & Scale factor \\
\hline CMUP & $0.716 \pm 0.013$ & $0.7521 \pm 0.0024$ & $0.952 \pm 0.017$ \\
CMX & $0.9828 \pm 0.0076$ & $0.99577 \pm 0.00056$ & $0.9870 \pm 0.0076$ \\
CMX (AR) & $0.9987 \pm 0.0053$ & $0.99750 \pm 0.00048$ & $1.0012 \pm 0.0053$ \\
CMX (KS) & $1.00 \pm 0.00$ & $0.9885 \pm 0.0044$ & $1.0117 \pm 0.0045$ \\
CMX (MS) & $0.906 \pm 0.032$ & $0.9896 \pm 0.0021$ & $0.916 \pm 0.032$ \\
\hline \hline
\end{tabular}

Table A.85: Muon reconstruction efficiencies and scale factors in CMUP-triggered events for 0i data set. (AR : Arch, MS : Miniskirt, KS : Keystone)

\begin{tabular}{cccc}
\hline \hline Stub & Data Efficiency & MC Efficiency & Scale factor \\
\hline CMUP & $0.7296 \pm 0.0079$ & $0.7616 \pm 0.0015$ & $0.958 \pm 0.011$ \\
CMX & $0.9595 \pm 0.0063$ & $0.99574 \pm 0.00035$ & $0.9636 \pm 0.0064$ \\
CMX (AR) & $0.9901 \pm 0.0056$ & $0.99755 \pm 0.00031$ & $0.9925 \pm 0.0056$ \\
CMX (KS) & $0.984 \pm 0.016$ & $0.9891 \pm 0.0025$ & $0.995 \pm 0.016$ \\
CMX (MS) & $0.842 \pm 0.022$ & $0.9907 \pm 0.0012$ & $0.850 \pm 0.022$ \\
\hline \hline
\end{tabular}

Table A.86: Muon reconstruction efficiencies and scale factors in CMX-triggered events for 0i data set. (AR : Arch, MS : Miniskirt, KS : Keystone)

\begin{tabular}{cccc}
\hline \hline Stub & Data Efficiency & MC Efficiency & Scale factor \\
\hline CMUP & $0.707 \pm 0.011$ & $0.7571 \pm 0.0020$ & $0.934 \pm 0.014$ \\
CMX & $0.9815 \pm 0.0060$ & $0.99600 \pm 0.00044$ & $0.9854 \pm 0.0061$ \\
CMX (AR) & $0.9907 \pm 0.0060$ & $0.99795 \pm 0.00035$ & $0.9927 \pm 0.0060$ \\
CMX (KS) & $1.00 \pm 0.00$ & $0.9938 \pm 0.0028$ & $1.0062 \pm 0.0028$ \\
CMX (MS) & $0.936 \pm 0.021$ & $0.9874 \pm 0.0019$ & $0.948 \pm 0.021$ \\
\hline \hline
\end{tabular}


Table A.87: Muon reconstruction efficiencies and scale factors in CMUP-triggered events for $0 \mathrm{j}$ data set. (AR : Arch, MS : Miniskirt, KS : Keystone)

\begin{tabular}{cccc}
\hline \hline Stub & Data Efficiency & MC Efficiency & Scale factor \\
\hline CMUP & $0.7128 \pm 0.0070$ & $0.7602 \pm 0.0013$ & $0.9376 \pm 0.0094$ \\
CMX & $0.9679 \pm 0.0050$ & $0.99562 \pm 0.00029$ & $0.9721 \pm 0.0050$ \\
CMX (AR) & $0.9970 \pm 0.0041$ & $0.99748 \pm 0.00026$ & $0.9995 \pm 0.0042$ \\
CMX (KS) & $0.964 \pm 0.020$ & $0.9864 \pm 0.0023$ & $0.978 \pm 0.021$ \\
CMX (MS) & $0.843 \pm 0.020$ & $0.99096 \pm 0.00094$ & $0.851 \pm 0.020$ \\
\hline \hline
\end{tabular}

Table A.88: Muon reconstruction efficiencies and scale factors in CMX-triggered events for $0 \mathrm{j}$ data set. (AR : Arch, MS : Miniskirt, KS : Keystone)

\begin{tabular}{cccc}
\hline \hline Stub & Data Efficiency & MC Efficiency & Scale factor \\
\hline CMUP & $0.7348 \pm 0.0088$ & $0.7566 \pm 0.0016$ & $0.971 \pm 0.012$ \\
CMX & $0.9727 \pm 0.0058$ & $0.99583 \pm 0.00036$ & $0.9768 \pm 0.0059$ \\
CMX (AR) & $0.9926 \pm 0.0053$ & $0.99767 \pm 0.00030$ & $0.9949 \pm 0.0053$ \\
CMX (KS) & $0.974 \pm 0.025$ & $0.9898 \pm 0.0029$ & $0.984 \pm 0.026$ \\
CMX (MS) & $0.881 \pm 0.022$ & $0.9886 \pm 0.0014$ & $0.891 \pm 0.022$ \\
\hline \hline
\end{tabular}

Table A.89: Muon reconstruction efficiencies and scale factors in CMUP-triggered events for 0k data set. (AR : Arch, MS : Miniskirt, KS : Keystone)

\begin{tabular}{cccc}
\hline \hline Stub & Data Efficiency & MC Efficiency & Scale factor \\
\hline CMUP & $0.7106 \pm 0.0095$ & $0.7582 \pm 0.0014$ & $0.937 \pm 0.013$ \\
CMX & $0.9599 \pm 0.0078$ & $0.99499 \pm 0.00034$ & $0.9647 \pm 0.0078$ \\
CMX (AR) & $0.9735 \pm 0.0085$ & $0.99744 \pm 0.00028$ & $0.9760 \pm 0.0085$ \\
CMX (KS) & $0.978 \pm 0.022$ & $0.9885 \pm 0.0023$ & $0.989 \pm 0.022$ \\
CMX (MS) & $0.902 \pm 0.022$ & $0.9875 \pm 0.0012$ & $0.914 \pm 0.022$ \\
\hline \hline
\end{tabular}


Table A.90: Muon reconstruction efficiencies and scale factors in CMX-triggered events for $0 \mathrm{k}$ data set. (AR : Arch, MS : Miniskirt, KS : Keystone)

\begin{tabular}{cccc}
\hline \hline Stub & Data Efficiency & MC Efficiency & Scale factor \\
\hline CMUP & $0.688 \pm 0.013$ & $0.7561 \pm 0.0018$ & $0.910 \pm 0.017$ \\
CMX & $0.9668 \pm 0.0086$ & $0.99660 \pm 0.00036$ & $0.9701 \pm 0.0087$ \\
CMX (AR) & $0.9833 \pm 0.0090$ & $0.99768 \pm 0.00033$ & $0.9855 \pm 0.0090$ \\
CMX (KS) & $0.933 \pm 0.046$ & $0.9925 \pm 0.0027$ & $0.940 \pm 0.046$ \\
CMX (MS) & $0.898 \pm 0.027$ & $0.9923 \pm 0.0013$ & $0.905 \pm 0.027$ \\
\hline \hline
\end{tabular}

Table A.91: Muon reconstruction efficiencies and scale factors in CMUP-triggered events for 0m data set. (AR : Arch, MS : Miniskirt, KS : Keystone)

\begin{tabular}{cccc}
\hline \hline Stub & Data Efficiency & MC Efficiency & Scale factor \\
\hline CMUP & $0.6898 \pm 0.0042$ & $0.7582 \pm 0.0014$ & $0.9098 \pm 0.0057$ \\
CMX & $0.9602 \pm 0.0034$ & $0.99499 \pm 0.00034$ & $0.9650 \pm 0.0034$ \\
CMX (AR) & $0.9813 \pm 0.0034$ & $0.99744 \pm 0.00028$ & $0.9838 \pm 0.0034$ \\
CMX (KS) & $0.882 \pm 0.019$ & $0.9885 \pm 0.0023$ & $0.892 \pm 0.019$ \\
CMX (MS) & $0.889 \pm 0.011$ & $0.9875 \pm 0.0012$ & $0.900 \pm 0.011$ \\
\hline \hline
\end{tabular}

Table A.92: Muon reconstruction efficiencies and scale factors in CMX-triggered events for Om data set. (AR : Arch, MS : Miniskirt, KS : Keystone)

\begin{tabular}{cccc}
\hline \hline Stub & Data Efficiency & MC Efficiency & Scale factor \\
\hline CMUP & $0.6954 \pm 0.0051$ & $0.7561 \pm 0.0018$ & $0.9197 \pm 0.0071$ \\
CMX & $0.9568 \pm 0.0038$ & $0.99660 \pm 0.00036$ & $0.9601 \pm 0.0039$ \\
CMX (AR) & $0.9754 \pm 0.0036$ & $0.99768 \pm 0.00033$ & $0.9777 \pm 0.0037$ \\
CMX (KS) & $0.856 \pm 0.031$ & $0.9925 \pm 0.0027$ & $0.862 \pm 0.031$ \\
CMX (MS) & $0.887 \pm 0.013$ & $0.9923 \pm 0.0013$ & $0.894 \pm 0.013$ \\
\hline \hline
\end{tabular}


Table A.93: Muon reconstruction efficiencies and scale factors in CMUP-triggered events for 0p data set. (AR : Arch, MS : Miniskirt, KS : Keystone)

\begin{tabular}{cccc}
\hline \hline Stub & Data Efficiency & MC Efficiency & Scale factor \\
\hline CMUP & $0.6802 \pm 0.0044$ & $0.7582 \pm 0.0014$ & $0.8972 \pm 0.0060$ \\
CMX & $0.9530 \pm 0.0039$ & $0.99499 \pm 0.00034$ & $0.9578 \pm 0.0039$ \\
CMX (AR) & $0.9744 \pm 0.0038$ & $0.99744 \pm 0.00028$ & $0.9769 \pm 0.0039$ \\
CMX (KS) & $0.894 \pm 0.019$ & $0.9885 \pm 0.0023$ & $0.905 \pm 0.019$ \\
CMX (MS) & $0.869 \pm 0.013$ & $0.9875 \pm 0.0012$ & $0.880 \pm 0.013$ \\
\hline \hline
\end{tabular}

Table A.94: Muon reconstruction efficiencies and scale factors in CMX-triggered events for Op data set. (AR : Arch, MS : Miniskirt, KS : Keystone)

\begin{tabular}{cccc}
\hline \hline Stub & Data Efficiency & MC Efficiency & Scale factor \\
\hline CMUP & $0.6869 \pm 0.0055$ & $0.7561 \pm 0.0018$ & $0.9085 \pm 0.0076$ \\
CMX & $0.9588 \pm 0.0041$ & $0.99660 \pm 0.00036$ & $0.9621 \pm 0.0042$ \\
CMX (AR) & $0.9762 \pm 0.0040$ & $0.99768 \pm 0.00033$ & $0.9784 \pm 0.0040$ \\
CMX (KS) & $0.954 \pm 0.028$ & $0.9925 \pm 0.0027$ & $0.961 \pm 0.028$ \\
CMX (MS) & $0.865 \pm 0.015$ & $0.9923 \pm 0.0013$ & $0.871 \pm 0.016$ \\
\hline \hline
\end{tabular}




\section{Appendix B}

\section{Event Display}
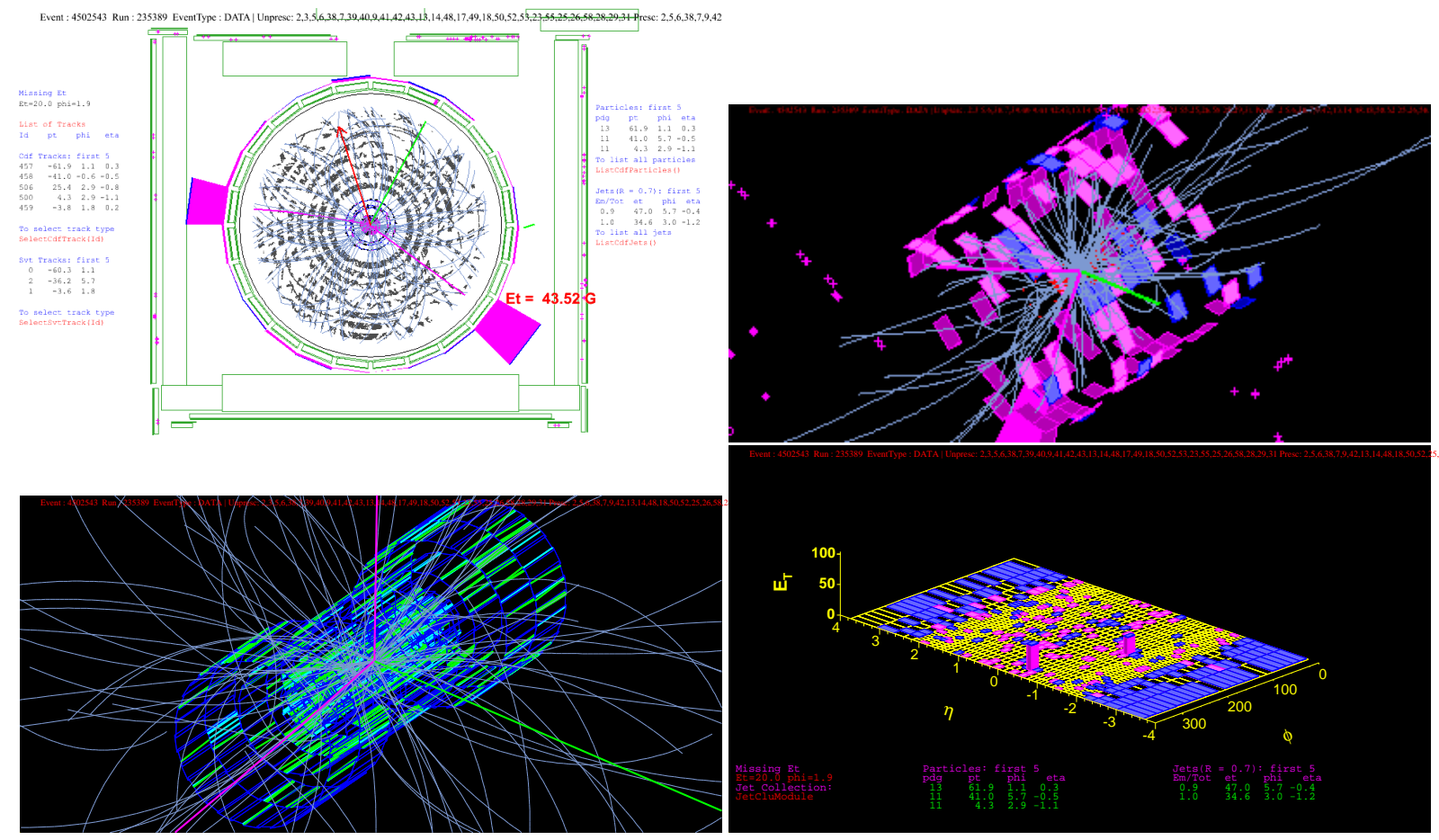

Figure B.1: Event Display of the $W Z$ candidate event with BDT Output $=0.89$ trained by the Higgs boson mass of $110 \mathrm{GeV} / c^{2}$. (top left: COT, top right: Calorimeter, bottom left: SVX, bottom right: Calorimter $\left.E_{T}\right)$. 

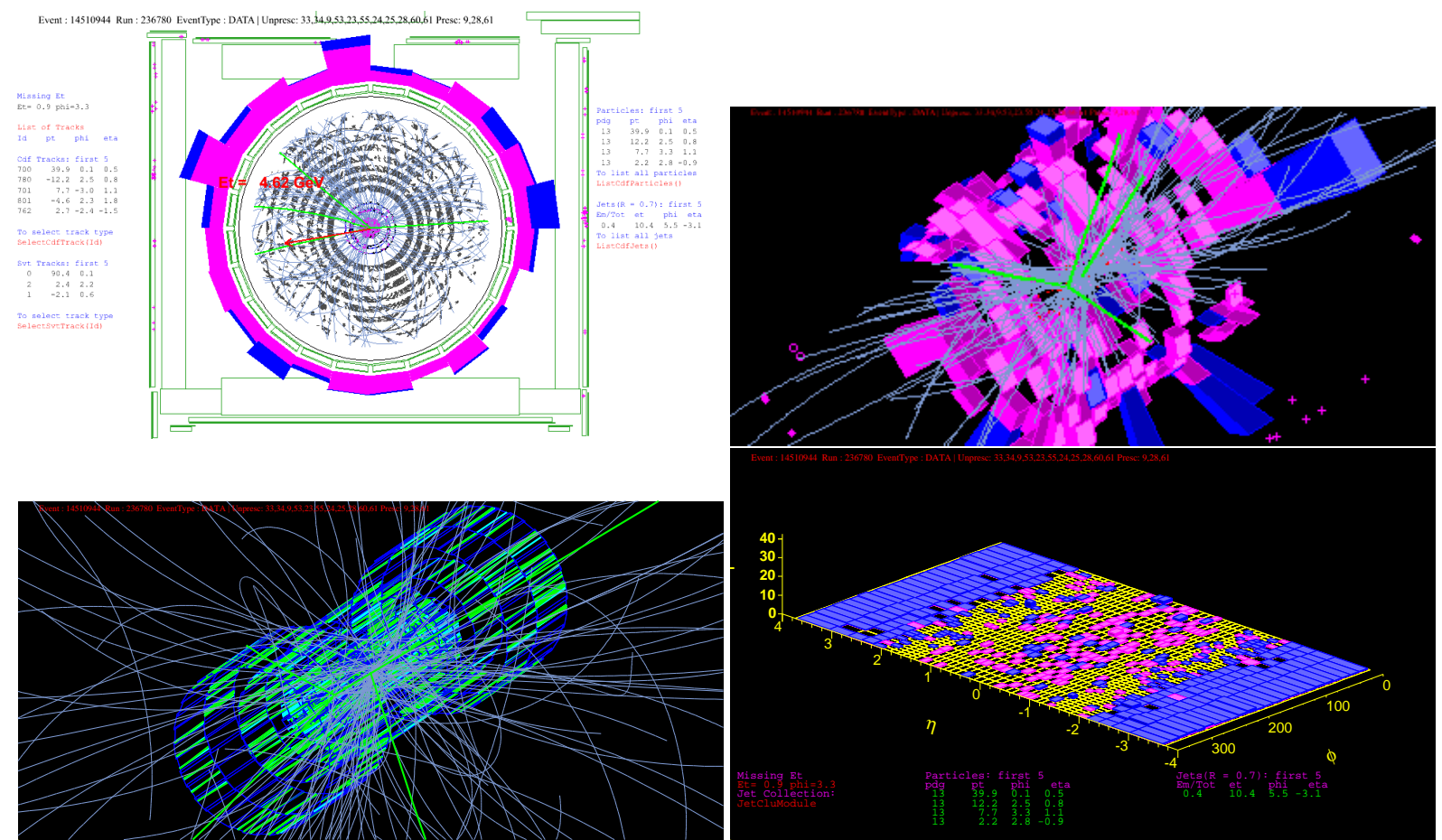

Figure B.2: Event Display of the fake candidate event with BDT Output=-0.90 trained by the Higgs boson mass of $110 \mathrm{GeV} / c^{2}$. (top left: COT, top right: Calorimeter, bottom left: SVX, bottom right: Calorimter $\left.E_{T}\right)$. 

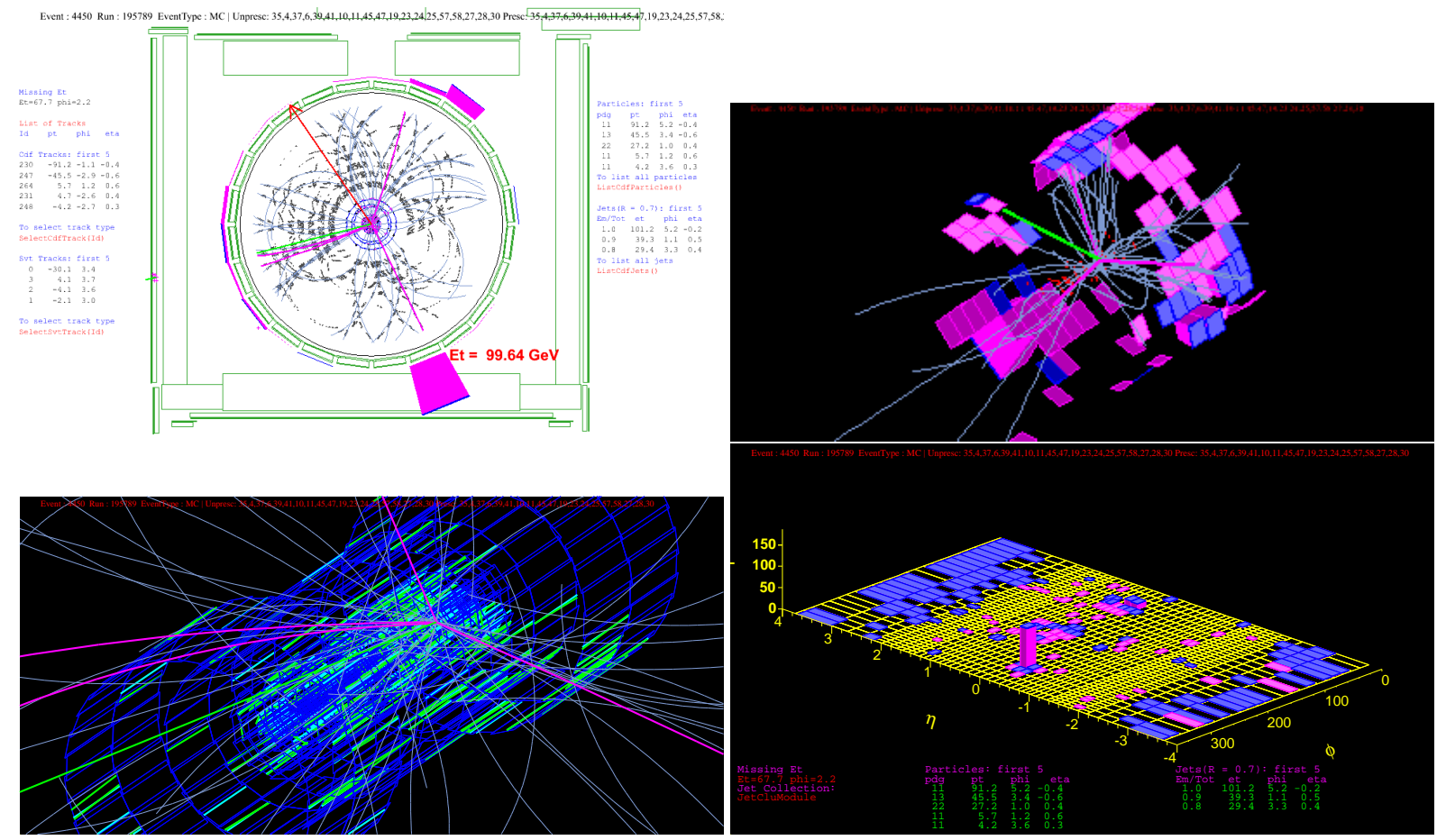

Figure B.3: Event Display of the $W Z$ Monte Carlo simulation with BDT Ouftput $=0.92$ trained by the Higgs boson mass of $110 \mathrm{GeV} / c^{2}$. (top left: COT, top right: Calorimeter, bottom left: SVX, bottom right: Calorimter $E_{T}$ ). 

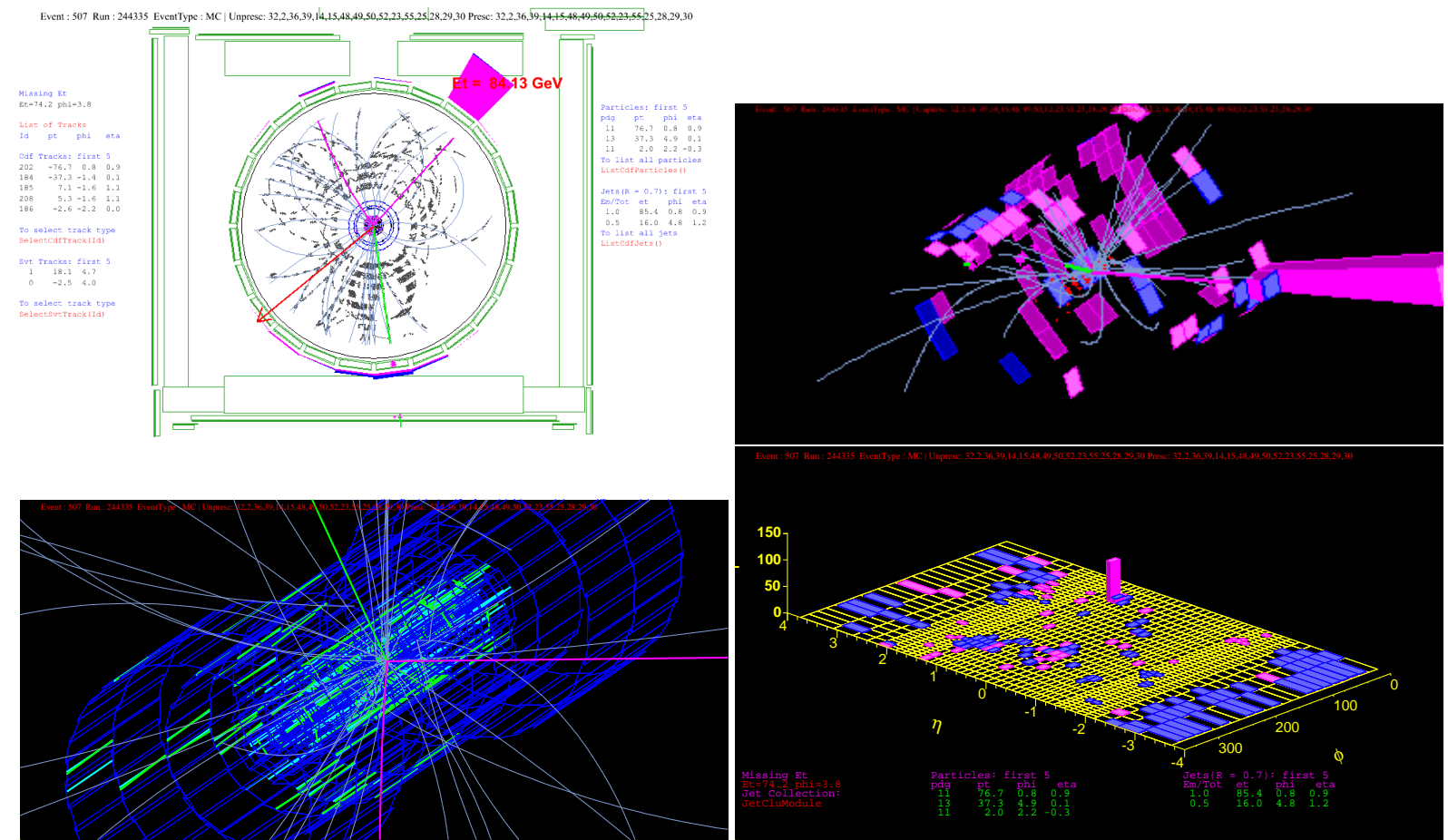

Figure B.4: Event Display of the Higgs Monte Carlo simulation $\left(m_{H}=110 \mathrm{GeV} / c^{2}\right)$ with BDT Ouftput $=0.92$ trained by the Higgs boson mass of $110 \mathrm{GeV} / c^{2}$. (top left: COT, top right: Calorimeter, bottom left: SVX, bottom right: Calorimter $E_{T}$ ). 


\section{Bibliography}

[1] J. Beringer et al. (Particle Data Group), "Review of Particle Physics", Phys. Rev. D 86, 010001 (2012)

[2] F. Halzen and A.D. Martin, "Quarks and Leptons: An Introductory Course in Modern Particle Physics", ISBN 0-471-88741-2 (1984)

[3] S. Glashow, "Partial Symmetries of Weak Interactions", Nucl. Phys. 22 (1961) 579.

[4] S. Weinberg, "A Model of Leptons", Phys. Rev. Lett. 19 (1967) 1264.

[5] A. Salam, in "Elementary Particle Theory", ed N. Svartholm, Almqvist and Wiksells, Stockholm (1969) p.367.

[6] P.W. Higgs, "Broken Symmetries, Massless Particles and Gauge Fields", Phys. Lett. 12 (1964) 132-133.

[7] P.W. Higgs, "Broken Symmetries and The Masses of Gauge Bosons", Phys. Rev. 13 (1964) 508-509.

[8] P.W. Higgs, "Spontaneous Symmetry Breakdown without Massless Bosons", Phys. Rev. 145 (1966) 1156-1163.

[9] H.E. Haber, G.L. Kane, and T. Sterling, "THE FERMION MASS SCALE POSSIBLE EFFECTS OF HIGGS BOSONS ON EXPERIMENTAL OBSERVABLES", Nucl. Phys. B161 (1979) 493-532.

[10] J.F. Gunion, H.E. Haber, G. Kane, and S. Dawson, "The Higgs Hunter's Guide", ISBN: 0-7382-0305-X (2000)

[11] J.F. Gunion and H.E. Haber, "CP-conserving two-Higgs-doublets model: The approach to the decoupling limit", Phys. Rev. D 67 (2003) 075019.

[12] M. Carena, J.S. Conway, H.E. Havber, and J.D. Hobbs, "Report of the Tevatron Higgs Working Group", arXiv:hep-ph/0010338.

[13] A. Djouadi, "The Anatomy of Electro-Weak Symmetry Breaking TomeI: The Higgs boson in the Standard Model", arXiv:hep-ph/0503172. 
[14] P.C. Bhat, H.B. Prosper, and S.S. Snyder, "Top Quark Physics At The Tevatron", arXiv:hep-ex/9809011.

[15] The ALEPH, CDF, DØ, DELPHI, L3, OPAL, SLD Collaboration, the LEP Electroweak working Group, the Tevatron Electroweak Group, and the SLD electroweak ans heavy flavour groups, "Precision Electroweak Measurements and Constraints on the Standard Model", arXiv:0811.4682 [hep-ex]

[16] The LEP Higgs Working Group, ALEPH Collaboration, DELPHI Collaboration, L3 Collaboration, and OPAL Collaboration, "Search for the Standard Model Higgs boson at LEP", Phys. Lett. B 565 (2003) 61-75.

[17] The LEP Higgs Working Group, ALEPH Collaboration, DELPHI Collaboration, L3 Collaboration, and OPAL Collaboration, "Searches for Higgs Bosons Decaying into Photons: Combined Results from the LEP Experiments", LHWG Note 200202 .

[18] CDF Collaboration, "Combination of CDF's searches for the standard model Higgs boson with up to $10.0 \mathrm{fb}^{-1}$ of data", CDF Public Note 10804 (2012).

[19] CDF Collaboration, "Search for Higgs Boson Produced in Association with a Vector Boson Using Like-Sign Dilepton Events in Run-II with $9.4 \mathrm{fb}^{-1}$ ", CDF Public Note 7307 (2012).

[20] CDF Collaboration, "Search for $\mathrm{H} \rightarrow \mathrm{WW}^{*}$ Production at CDF Using $9.7 \mathrm{fb}^{-1}$ of Data", CDF Public Note 10760 (2012).

[21] T. Aaltonen, D. Yamato, et al. (CDF Collaboration), "Combined search for the standard model Higgs boson decaying to a $b \bar{b}$ pair using the full CDF data set", Phys. Rev. Lett. 109, 111802 (2012).

[22] T. Aaltonen, D. Yamato, et al. (CDF Collaboration), "Search for the standard model Higgs boson decaying to a $b \bar{b}$ pair in events with one charged lepton and large missing transverse energy using the full CDF data set", Phys. Rev. Lett. 109, 111804 (2012).

[23] T. Aaltonen, D. Yamato, et al. (CDF Collaboration), "Search for the standard model Higgs boson decaying to a $b \bar{b}$ pair in events with two oppositely-charged leptons using the full CDF data set", Phys. Rev. Lett. 109, 111803 (2012).

[24] T. Aaltonen, D. Yamato, et al. (CDF Collaboration), "Search for the standard model Higgs boson decaying to a $b \bar{b}$ pair in events with no charged leptons and large missing transverse energy using the full CDF data set", Phys. Rev. Lett. 109, 111805 (2012).

[25] CDF Collaboration, "Search for the Standard Model Higgs Boson in $\tau^{+} \tau^{-}+$jets final state with $8.3 \mathrm{fb}^{-1}$ of CDF data" CDF Public Note 10625 (2011). 
[26] T. Aaltonen, D. Yamato, et al. (CDF Collaboration), "An includsive search for the Higgs boson in the four-lepton final state at CDF", Phys. Rev. D 86, 091101(R) (2012).

[27] T. Aaltonen, D. Yamato, et al. (CDF Collaboration), "Search for a Higgs boson in the diphoton final state using the full CDF data set from $p \bar{p}$ collisions at $\sqrt{s}=1.96$ TeV", Phys. Lett. B 717, 173-181 (2012).

[28] CDF Collaboration, "A search for the Higgs Boson in the All Hadronic Channel using $9.45 \mathrm{fb}^{-1}$ of CDF data", CDF Public Note 10792 (2012).

[29] T. Aaltonen, D. Yamato, et al. (CDF Collaboration), "Search for the standard model Higgs boson produced in association with top quarks using the full CDF data set", Phys. Rev. Lett. 109, 181802 (2012).

[30] DØ Collaboration, "Updated Combination of Searches for the Standard Model Higgs Boson at the DØ Experiment in $9.7 \mathrm{fb}^{-1}$ of Data", arXiv:hep-ex/1207.0422.

[31] DØ Collaboration, "Updated search for Higgs boson production in final states with a lepton, missing energy, and at least two jets in $9.7 \mathrm{fb}^{-1}$ of Tevatron data", DØ Note 6346-CONF (2012).

[32] DØ Collaboration, "Updated search for $Z H \rightarrow \ell^{+} \ell^{-} b \bar{b}$ production in $9.7 \mathrm{fb}^{-1}$ of $p \bar{p}$ collisions", DØ Note 6342-CONF (2012).

[33] DØ Collaboration, "Updated Search for the Standard-Model Higgs Boson in the $Z H \rightarrow \nu \bar{\nu} b \bar{b}$ Channel in $9.5 \mathrm{fb}^{-1}$ of $p \bar{p}$ Collisions at $\sqrt{s}=1.96 \mathrm{TeV}$ ", DØ Note 6340-CONF (2012).

[34] DØ Collaboration, "Updated search for Higgs boson production in dilepton plus missing transverse energy final states with $9.7 \mathrm{fb}^{-1}$ of $p \bar{p}$ collisions at $\sqrt{s}=1.96$ TeV", DØ Note 6343-CONF (2012).

[35] DØ Collaboration, "Updated search for the Standard Model Higgs Boson in the $\gamma \gamma+X$ final state at D0", DØ Note 6345-CONF (2012).

[36] DØ Collaboration, "Updated search for the Standard Model Higgs Boson in Final States with three Leptons and missing transverse energy with $9.7 \mathrm{fb}^{-1}$ of $p \bar{p}$ collisions at $\sqrt{s}=1.96 \mathrm{TeV} "$, D Note 6347-CONF (2012).

[37] D $\varnothing$ Collaboration, "Search for Associated Higgs Boson Production $V H \rightarrow e^{ \pm} \nu_{e} \mu^{ \pm} \nu_{\mu}$ with Like Charged Electron Muon pairs using $9.7 \mathrm{fb}^{-1}$ of $p \bar{p}$ Collisions at $\sqrt{s}=1.96 \mathrm{TeV} ", \mathrm{D} \varnothing$ Note 6301-CONF (2012).

[38] DØ Collaboration, "Search for the standard model Higgs boson in the $\tau \tau \mu+X$ final state in $7.0 \mathrm{fb}^{-1}$ of $p \bar{p}$ collisions at $\sqrt{s}=1.96 \mathrm{TeV} "$, D $\varnothing$ Note 6286 -CONF (2012). 
[39] CDF Collaboration, DØ Collaboration, Tevatron New Physics, Higgs Working Group, "Updated Combination of CDF and D0 Searches for Standard Model Higgs Boson Production with up to $10.0 \mathrm{fb}^{-1}$ of Data", arXiv:1207.0449v2 [hepex] (2012).

[40] T. Aaltonen, D. Yamato, et al. (CDF, DØ Collaboration), "Evedence for a particle produced in association with weak bosons and decaying to a bottomantibottom quark pair in Higgs boson searches at the Tevatron", Phys. Rev. Lett. 109, 071804 (2012).

[41] D $\varnothing$ Collaboration, "Search for a Fermiophobic Higgs Boson in the di-photon final state using $9.7 \mathrm{fb}^{-1}$ of DØ data", DØ Note 6297-CONF (2012).

[42] G. Aad, et al. (ATLAS Collaboration), "Observation of a new particle in the search for the Standard Model Higgs boson with the ATLAS detector at the LHC", Phys. Lett. B 716 (2012) 1-29.

[43] S. Chatrchyan, et al. (CMS Collaboration), "Observation of a new boson at a mass of $125 \mathrm{GeV}$ with the CMS experiment at the LHC", Phys. Lett. B 716 (2012) 30-61.

[44] The CDF II Collaboration, "The CDF II Detector Technical Design Report", FERMILAB-Pub-96/390-E (1996).

[45] T.K. Nelson et al, "The CDF Layer 00 Detector", FERMILAB-CONF-01/357-E (2001).

[46] C.S. Hill et al, "Initial experience with the CDF layer 00 silicon detector", Nucl. Instrum. and Meth. A 511 (2003) 118-120.

[47] A. Sill et al, "CDF Run II silicon tracking projects", Nucl. Instrum. and Meth. A 447 (200) 1-8.

[48] W. Ashmanskas et al, "The CDF silicon vertex trigger", Nucl. Instrum. and Meth. A 518 (2004) 532-636.

[49] A. Affolder et al, "Status report of the Intermediate Silicon Layers detector at CDFII", CDF Public Note 5687 (2001).

[50] A. Affolder et al, "Intermediate silicon layers detector for the CDF experiment", Nucl. Instrum. and Meth. A 453 (2000) 84-88.

[51] T. Affolder et al, "CDF Central Outer Tracker", CDF Public Note 6267 (2003).

[52] L. Balka et al, "The CDF Central Electromagnetic Calorimeter", Nucl. Instrum. Meth. A 267, 272 (1988). 
[53] S. Lami et al "The CDF calorimeter upgrade for Run IIb", Nucl. Instrum. Meth. A 518 (2004) 39-41.

[54] S. Bertolucci et al, "The CDF Central and Endwall Hadron Calorimeter", Nucl. Instrum. Meth. A 267, 301 (1988).

[55] Y. Seiya et al, "The CDF plug upgrade electromagnetic calorimeter test beam results", Nucl. Instrum. Meth. A 480 (2002) 524-546.

[56] D. Acosta et al, "The CDF Cherenkov luminosity monitor", Nucl. Instrum. Meth. A 461 (2001) 540-544.

[57] D. Acosta et al, "The performance of the CDF luminosity monitor", Nucl. Instrum. Meth. A 494 (2002) 57-62.

[58] Takayuki Wakisaka, "Search for the Wh Production Using High-p ${ }_{T}$ Isolated LikeSign Dilepton Events in 1.96-TeV Proton-Antiproton Collisions" , Ph.D Thesis, Osaka City University.

[59] V.Tiwari, G.Giurgiu, M.Paulini, J.Russ, and B.Wicklund, "Likelihood Based Electron Tagging", CDF Public Note 7121.

[60] A. Bhatti et al, "Determination of the Jet Energy Scale as the Collider Detector at Fermilab", hep-ex/0510047v1.

[61] B. Rossi, "High Energy Particles", Prentice Hall, 1965.

[62] Torbjörn Sjöstrand, Stephen Mrenna, Peter Skands, "PYTHIA 6.4 Physics and Manual", FERMILAB-PUB-06-052-CD-T, 2006.

[63] http://geant4.cern.ch/.

[64] L.Breiman, J.H.Friedman, R.A.Olshen and C.J.Stone "Classification and regression trees", ISBN: 0-412-04841-8

[65] H.Yang, B.P.Roe and J.Zhu. "Studies of Boosted Decision Trees for MiniBooNE Particle Identification", arXiv:physics/0508045

[66] The ROOT team, "ROOT An Object-Oriented Data Analysis Framework: Users Guide 5.26", (2009)

[67] A.Hocker, P.Speckmayer, J.Stelzer, J.Therhaag, E.von Toerne, H.Voss, "TMVA 4 Toolkit for Multivariate Data Analysis with ROOT Users Guide", CERN-OPEN2007-007 (2009).

[68] K.A.Assamgan et al, "THE HIGGS WORKING GROUP: Summary Report", hep-ph/0406152v1. 
[69] J.M. Campbell and R.K. Ellis, "An update on vector boson pair production at hadron colliders", Phys. Rev. D 60113006 (1999).

[70] CDF Collaboration, D. Acosta, et al, "Search for Electroweak single-top-quark production in $p \bar{p}$ collisions at $\sqrt{s}=1.96$ TeV", Phys. Rev. D 71, 012005 (2005). 